\title{
QUANTITATIVE MAPPING OF NANOTHERMAL TRANSPORT via SCANNING THERMAL Microscopy
}

\author{
Jean Spièce
}

THESIS SUBMITTED FOR THE DEGREE OF

Doctor OF Philosophy

\section{Lancaster}

\author{
DePaRtMent of Physics \\ LANCASTER UNIVERSITY \\ LANCASTER, UK
}

September 26, 2018 
O Freunde, nicht diese Töne!

Sondern laßt uns angenehmere anstimmen, und freudenvollere.

Freude!

Freude!

Freude, schöner Götterfunken

Tochter aus Elysium,

Wir betreten feuertrunken,

Himmlische, dein Heiligtum!

Deine Zauber binden wieder

Was die Mode streng geteilt;

Alle Menschen werden Brüder,

Wo dein sanfter Flügel weilt.
Oh friends, not these sounds!

Let us instead strike up more pleasing and more joyful ones!

Joy!

Joy!

Joy, beautiful spark of divinity, Daughter from Elysium,

We enter, burning with fervour, heavenly being, your sanctuary!

Your magic brings together what custom has sternly divided. All men shall become brothers, wherever your gentle wings hover.

Ode to Joy, von Schiller and Beethoven 


\section{ACKNOWLEDGEMENTS}

This thesis is the fruit of many coincidences and collaborations without which it wouldn't have been born. So therefore, there shouldn't be any particular order of importance such as in a puzzle, removing one piece makes the picture incomplete. The contributions and impact of each of the following people cannot be completely pictured in few words, therefore the easiest is just thank you all.

Right so, always there to provide me with endless support, trust and energy, my supervisor Oleg Kolosov has been at the core of my work since the first days, and even before that. Long discussions, late emails and endless nights playing in the lab, he taught me more I'll probably ever realise, beyond the sole scientific realm.

Then, I must acknowledge the limitless impact that Pambos Evangeli had on my life since his first appearance in the lab, as a friend and mentor in many respects. Long hours working together on the vacuum system, long hours through the QUANTIHEAT maze, long hours laughing wherever, such memories will accompany me for the rest of my life.

Also helping me for in the quest of a greater SThM, Ben Robinson as well as Alex Robson played a major role in our QUANTIHEAT and SThM success. From the marvellous spreadsheets and management skills to the perfect sample polishing, their guidance and help were always fruitful. Cheers.

When coming to QUANTIHEAT, I would like to thank all people who have worked towards its realisation. During the course of the project, the $20+$ partners of the consortium brought me, through direct or indirect discussions, ideas and understanding that are underlying this work. In particular, I must thank Séverine Gomès and Sylvie Rault, the best project coordinator and manager I know. Also, Alexandros El Sachat and Francesc Alzina for the fruitful, still ongoing, collaborations. Odile Bezencenet and her Thales partners for the great discussions and always motivating and challenging samples. Abeer Al Mohtar for the good fun and the endless hope for publishing a paper. From Glasgow, our closest SThM neighbours, Rory Lambert, Phil Dobson and John Weaver as well as Gordon Mills, their deep understanding of the probe behaviour provided us with countless useful information.

I must as well acknowledge the rest of the Lancaster Nanoscale Characterisation group. Peter Tovee for passing me the SThM baton. Nick Kay for his support on the many nanomechanical systems. Ghazi Al Sharif for teaching me few words of Arabic. The Freshers, Elisaja Castanon and Martaja San Juan, for keeping the boss busy and teaching us millennial Spanish culture. Thanks to my fellow PhD friends Eva and Denise and the Italian mafia Angelo and Leo.

Few collaborations started outside of the QUANTIHEAT consortium thanks to Oleg never ending playful thrill for new samples. From Stuttgart, bringing great samples in her bag, I thank Linda Haenel for being always precise and dedicated 
during her stay in Lancaster, our results are amongst my favourites. Formerly from Stuttgart as well, now from Oxford and Delft, Achim Harzheim and Pascal Gehring, I thank them for the great curious samples they brought us, especially the penultimate ones. Their machine-like skills will always be remembered. From York, Siew Wai Poon and Sarah Thompson were at the start of the combination of SThM and BEXP, they also helped understanding and simplifying some procedures.

Then, I have to acknowledge Lancaster University for the great infrastructures, the physics workshops and Steve Holden from the physics store. The department staff for always ensuring safe working conditions and efficient management. A special thought must go to the many people who made the refurbishment possible, the old Soviet style labs needed it. Thanks also to the brilliant Physics Garden and the Design the Spine program for having as many bricks as characters in this thesis and always being a good discussion subject.

I also need to acknowledge the Golden Coffee club for must needed decompressing breaks and beyond: Dr. Ramon Gavito, Dr. Kunal Lulla(l) and Dr. Charalambous. Furthermore, thanks to Elena, Ramon and the cat with no name who were always there to offer me shelter and a warm home during the last months of this thesis work.

For the great atmosphere in our house, thanks to the Gang Will, Ola and Max. Special thanks to my food crawling mate Will.

Penultimately, I thank my family for the support, understanding they provided me with. My mother and father, my sister, François and Félicien as well as Anne. These three and a half years have seen many changes, many losses and many gains. That's life. Also, a special thanks to Nani and Bonpi, Jean and Sophie for their incredible hosting abilities, for the exceptional driving abilities of the early days and for the confidence.

Finally, with burning love and incommensurable admiration, thanks to my other-half Marine. Love is in the air and everywhere.

Humble thoughts go to Beethoven and the 18 recording of his complete symphonies which accompanied me during most of this thesis writing. 


\section{DECLARATION}

This thesis is my original work and has not been submitted, in whole or in part, for a degree at this or any other university. Nor does it contain, to the best of my knowledge and belief, any material published or written by another person, except as acknowledged in the text. 


\section{LIST OF PUBLICATIONS}

Peer-reviewed papers and conference proceedings:

- Probing thermal transport and layering in disk media, S.W Poon, J. Spièce, A.J. Robson, O.V. Kolosov, S. Thompson In: Magnetics Conference (INTERMAG), 2017 IEEE International.

- Field-effect control of graphene-fullerene thermoelectric nanodevices, P. Gehring, A. Harzheim, J. Spièce, Y. Sheng, G. Rogers, C. Evangeli, A. Mishra, B.J. Robinson, K. Porfyrakis, J.H. Warner, O.V. Kolosov, G.A.D. Briggs, J.A. Mol In: Nanoletters, 17(11), 7055-7061, 2017.

- Thermal transport in epitaxial $\mathrm{Si}_{1-x} \mathrm{Ge}_{x}$ alloy nanowires with varying composition and morphology,

A. El Sachat, J.S. Reparaz, J. Spièce, M.I. Alonso, A.R. Goni, M. Garriga, P.O. Vaccaro, M.R. Wagner, O.V. Kolosov, C.M. Sotomayor Torres, F. Alzina In: Nanotechnology, 28(50), 505704, 2017.

- Improving accuracy of nanothermal measurements via spatially distributed scanning thermal microscope probes,

J. Spièce, C. Evangeli, K. Lulla, A.J. Robson, B.V. Robinson, O.V. Kolosov In: Journal of Applied Physics, 124(1), 015101, 2018.

Papers submitted:

- Geometrically enhanced thermoelectric effects in graphene nanoconstrictions,

A. Harzheim, J. Spièce, C. Evangeli, Y. Sheng, J.Warner, A. Briggs, J. Mol, P. Gehring, O.V. Kolosov. Submitted to Nanoletters.

Papers in preparation

- Nanothermal and nanomechanical characterization of bare and metal-covered block copolymer thin films,

A. El Sachat, J. Spièce, C. Evangeli, A.J. Robson, M. Kreuzer, E. Guen, P.-O. Chapuis, S. Gomès, C.M. Sotomayor Torres, O.V. Kolosov, F. Alzina. 
- Measuring thermal transport in buried nanostructures,

J. Spièce, C. Evangeli, A.J. Robson, A. El Sachat, L. Haenel, M.I. Alonso, M. Garriga, B.J. Robinson, M. Oehme, J. Schulz, F. Alzina, C. Sotomayor Torres, O.V. Kolosov.

- Scanning thermal microscopy of 2D materials at cryogenic temperatures,

C. Evangeli, J. Spièce, A. Molina-Mendoza, T. Mueller, O.V. Kolosov.

- Thermal transport in natural heterostructure: a nanoscale study,

C. Evangeli, J. Spièce, A. Molina-Mendoza, T. Mueller, O.V. Kolosov.

This work has also been presented at the following scientific conferences and workshops:

Lead presenter:

- Scanning thermal microscopy and finite elements studies of 2D materials, J. Spièce, B.J. Robinson, O.V. Kolosov, European Materials Research Society Spring Meeting, 2015, Lille, France. (Oral).

- Quantitative nanothermal study of 2D materials by SThM and finite elements simulations,

J. Spièce, B.J. Robinson, O.V. Kolosov, Graphene Week, 2015, Manchester, UK. (Poster).

- Cross-sectional characterization of thermal conductivity in multilayer semiconductor nanostructures - gradient SiGe alloys,

J Spièce, A.J. Robson, B.J. Robinson, F. Alzina, O.V. Kolosov, Materials for Advanced Metalization, 2016, Brussels, Belgium. (Poster).

- Multiparametrical investigation of confined underliquid systems,

J. Spièce, P. Tovee, B.J. Robinson, O.V. Kolosov, 5th Multifrequency AFM Conference, 2016, Madrid, Spain. (Oral). 
- Thermal characterization of cross-sectioned multilayer gradient SiGe alloys, J. Spièce, A.J. Robson, B.J. Robinson, A. El Sachat, M.I. Alonso, M. Garriga, F. Alzina, C. Sotomayor Torres, O.V. Kolosov, Eurotherm Seminar 108 Nanoscale and Microscale heat transfer, 2016, Santorini, Greece. (Poster).

- Comprehensive modelling of probe-sample heat transfer for ambient and vacuum conditions,

J. Spièce, C. Evangeli, A.J. Robson, B.J. Robinson, O.V. Kolosov, Eurotherm Seminar 108 Nanoscale and Microscale heat transfer, 2016, Santorini, Greece. (Oral).

- Structural characterisation of ALD coated porous Si via Beam-Exit CrossSectional Polishing,

A.J. Robson, J. Spièce, C. Evangeli, R. Ritasalo, M. Matvejeff, K. Grigoras, M. Prunnila, O.V. Kolosov, Microscopy and Microscience Congress, 2017, Manchester, UK. (Poster).

- Novel nanoscale method for thermal conductivity measurements, J. Spièce, A.J. Robson, C. Evangeli, O.V. Kolosov, Microscopy and Microscience Congress, 2017, Manchester, UK. (Poster).

Contributing author:

- Scanning thermal microscopy and finite elements studies of 2D materials, B.J. Robinson, J. Spièce, O.V. Kolosov, International Conference on Materials for Advanced Technologies, 2015, Singapore. (Oral).

- Nano-mapping of Surface and Subsurface Physical Properties of 2D materials,

O.V. Kolosov, N. Kay, J. Spièce, G. Alsharif, B.J. Robinson, F. Dinelli, ANM, 2016, Aveiro, Portugal. (Oral).

- Characterisation of local thermal properties in nanoscale structures by scanning thermal microscopy, 
E. Castanon, C. Evangeli, J. Spièce, B.J. Robinson, S. Gomès, O. Kazakova, O.V. Kolosov, Microscopy and Microscience Congress, 2017, Manchester, UK. (Poster).

- Scanning Thermal Microscopy on 2D Materials at cryogenic temperatures, C. Evangeli, J. Spièce, A.J. Robson, N. Kay, O.V. Kolosov, Microscopy and Microscience Congress, 2017, Manchester, UK. (Poster).

- Probing Nanoscale Heat Transport in Liquid Environments - Contact and Non-Contact Immersion Scanning Thermal Microscopy (iSThM)

OV. Kolosov, J. Spièce, B.J. Robinson, Materials Research Society Spring Meeting, 2017, Phoenix, USA. (Oral).

- Quantitative Measurements of Intrinsic Thermal Conductivity of Surface and Buried Nanoscale Layers via Cross-Sectional Scanning Thermal Microscopy - X-SThM,

J. Spièce, C. Evangeli, A.J. Robson, B.J. Robinson, F. Alzina, O.V. Kolosov, Materials Research Society, 201\%, Phoenix, USA. (Poster).

- Geometrically Enhanced Thermoelectric Effects in Graphene Nanoconstrictions,

A. Harzheim, J. Spièce, C. Evangeli, Y. Sheng, J. Warner, A. Briggs, J. Mol, P. Gehring, O.V. Kolosov, American Physical Society March Meeting 2018, Los Angeles, USA. (Oral).

- Scanning Thermal Microscopy of Thermal Interface Materials, J. Spièce, C. Evangeli, A.J. Robson, K. Lulla, B.J. Robinson, R. Aubry, S. Jus, L. Divay, A. Borta Boyon, O. Bezencenet, O.V. Kolosov, Materials Research Society Spring Meeting, 2018, Phoenix, USA. (Poster).

- Exploring 3D Thermal Properties of Nanomaterials, J. Spièce, C. Evangeli, A. El Sachat, L. Haenel, A.J. Robson K. Lulla, B.J. Robinson, M.I. Alonso, M. Garriga, M. Oehme, C. Sotomayor Torres, J. 
Schulze, F. Alzina, O.V. Kolosov, Materials Research Society Spring Meeting, 2018, Phoenix, USA. (Poster).

- Scanning Thermal Gate Microscopy (SThGM)-A New Tool for Nanoscale Exploration of Thermoelectric Phenomena,

J. Spièce, A. Harzheim, C. Evangeli, Y. Sheng, J. Warner, A. Briggs, J. Mol, P. Gehring, O.V. Kolosov, Materials Research Society Spring Meeting, 2018, Phoenix, USA. (Oral).

- Mapping Heat Transport in Van der Waals Nanostructures via Cryogenic Scanning Thermal Microscopy,

C. Evangeli, J. Spièce, B.J. Robinson, A. Molina-Mendoza, T. Mueller, O.V. Kolosov, Materials Research Society Spring Meeting, 2018, Phoenix, USA. (Poster). 


\section{ABSTRACT}

By expanding the limit of miniaturisation to achieve superior materials and devices properties, nanoscience and nanotechnology create new challenges to understand materials behaviour at the nanometre length scales. New phenomena require matching tools that in turn open new investigation perspectives, themselves launching platforms for new discoveries. Amongst the vast realm of nanoresearch, thermal properties have gained interest in the past decades due their crucial importance in technological developments, ranging from mainstream semiconductor microelectronics to cutting edge quantum technologies.

Whereas major standard techniques to measure thermal properties are not efficient for studying nanosstructured materials, Scanning Thermal Microscopy (SThM) offers both sensitivity to nanoscale thermal transport and a spatial resolution down to a few nanometres. This thesis develops fundamental aspects of the SThM technique by increasing its reproducibility and developing an experimental and analytical framework to analyse experimental data.

These developments are then applied to produce quantitative measurements of a wide range of materials from vertically aligned carbon nanotubes to metal covered block copolymers. In brief, we probed the 3D thermal properties distribution of isotropic and anisotropic materials, such as optoelectronic thin film. We also measured low dimensional systems of 2D materials heterostructures from franckeite and graphene on $\mathrm{MoS}_{2}$. Thermoelectric properties of graphene nanoconstrictions are unveiled using a combined three-terminal approach. Additionally, cryoSThM is introduced as a new tool with the ability to measure at temperatures below $150 \mathrm{~K}$

The research presented in this thesis has a two-fold impact. On one hand, major technical SThM challenges are answered and efficient solutions developed. In this respect, cryoSThM and 3D nanothermal probing of materials open radically new routes for further investigations. On the other hand, new insights are gained 
from the extraction of materials properties and the observation of new phenomena. The knowledge gained through this research leads to innovative keys to develop applications in various fields, ranging from heat management to thermoelectric energy conversion. 


\section{Contents}

$\begin{array}{ll}\text { List of Figures } & \text { xxii }\end{array}$

List of Tables $\quad$ xxiii

Glossary and Abbreviations $\quad$ xxiv

$\begin{array}{ll}\text { List of symbols } & \text { Xxv }\end{array}$

\begin{tabular}{lll}
\hline & Outline and motivations & 1
\end{tabular}

\begin{tabular}{lll}
\hline 2 & Background review & 3
\end{tabular}

$2.1 \quad$ Introduction . . . . . . . . . . . . . . . . . . . . 3

2.1 .1 Existing techniques and limitations . . . . . . . . . . . . 4

2.2 Heat conduction at the nanoscale . . . . . . . . . . . . . 6

$2.2 .1 \quad$ Diffusive transport and its nanoscale limit . . . . . . . . 7

2.2 .2 Transport at interfaces . . . . . . . . . . . . . . . 11

2.3 Scanning thermal microscopy $(\mathrm{SThM})$. . . . . . . . . . . . . . . . 12

$2.3 .0 \quad$ Scanning probe microscopy . . . . . . . . . . . . . . . . . . 13

$2.3 .1 \quad$ History and principles of SThM . . . . . . . . . . . . . . 15

$2.3 .2 \quad$ Basic operation possibilities . . . . . . . . . . . . 20

2.3 .3 Heat transfer mechanisms in the tip-sample system . . . . . 25

2.3 .4 What is measured? . . . . . . . . . . . . . . . . . . . . . 29

2.4 Modelling and simulations . . . . . . . . . . . . . . . . . 30

$2.4 .1 \quad$ Finite Element Analysis compared to analytical formulations 31

$2.4 .2 \quad$ Spreading resistance of layered systems . . . . . . . . . 32

2.5 Summary . . . . . . . . . . . . . . . . . . . . . 37

3 SThM experimental models and setups for exploring nanoscale $\begin{array}{ll}\text { heat transport } & 38\end{array}$

3.1 Precise nanothermal measurements via spatially distributed scan-

ning thermal microscope . . . . . . . . . . . . . . . . . . . . 38

$3.1 .1 \quad$ Introduction . . . . . . . . . . . . . . . . . . . . . . . . 38

3.1.2 Combined analytical model and high-precision SThM setup. 41

3.1 .3 Results and discussion . . . . . . . . . . . . . . . 50

3.1 .4 Summary . . . . . . . . . . . . . . . . . . . 55

3.2 Introducing cryoSThM - nanothermal transport measurements at cryogenic temperatures . . . . . . . . . . . . . 56

3.2.1 Vacuum SThM setup for measurements at cryogenic tem-

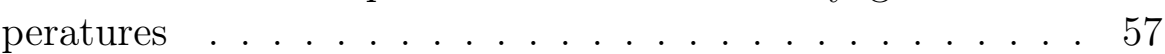

3.2 .2 Measurement model . . . . . . . . . . . . . . . . . 59 
$3.2 .3 \quad$ CryoSThM demonstration . . . . . . . . . . . . . . . . 62

3.2 .4 Summary $\ldots \ldots \ldots \ldots \ldots$

3.3 Dynamic SThM: quasi non-contact method for multiparametrical

investigation $\ldots \ldots \ldots \ldots \ldots \ldots \ldots \ldots$

3.3 .1 Introduction $\ldots \ldots \ldots \ldots \ldots$

3.3 .2 Methodology . . . . . . . . . . . . . . . . . 70

3.3 .3 Results . . . . . . . . . . . . . . . . . . . . . . 72

3.3 .4 Summary . . . . . . . . . . . . . . . . 75

4 Quantitative thermal transport measurements in nanostructures 76

4.1 Principles and applications of layer analytical model . . . . . . . 76

4.1 .1 Silicon oxide steps $\ldots \ldots \ldots \ldots \ldots$. . . . . . . . . . 81

$4.1 .2 \quad$ ALD aluminium oxide layers . . . . . . . . . . . . . . . . 82

4.2 Thermal transport in metal covered block copolymers . . . . . . . 83

4.3 Summary $\ldots \ldots \ldots \ldots \ldots \ldots$

5 Three dimensional mapping of thermal properties 95

5.1 Introduction . . . . . . . . . . . . . . . . . . . . 95

5.2 Combining BEXP and SThM . . . . . . . . . . . . 97

5.3 Quantitative measurements of thermal conductivity and interface

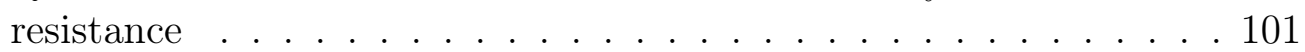

5.4 Thermal transport in anisotropic media . . . . . . . . . . . 105

5.5 Characterisation of Thermal Interface Materials . . . . . . . . . 109

5.5 .1 Buried morphology and structure . . . . . . . . . . . . 110

5.5 .2 Nanoscale thermal mapping of full interface . . . . . . . . 113

5.5.3 Perspectives on nanothermal characterisation of TIM . . . . 121

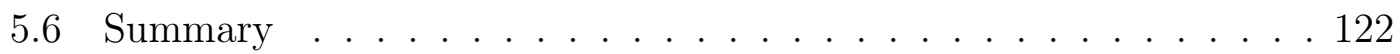

6 Nanoscale thermal transport in low dimensional materials 124

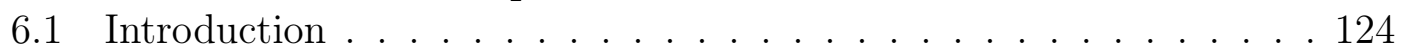

6.2 Heat transport in two dimensional materials and their heterostructures 124 $6.2 .1 \quad$ Franckeite $\ldots \ldots \ldots \ldots \ldots \ldots$

6.2 .2 Heterostructure of $\mathrm{MoS}_{2}$ on graphene . . . . . . . . . . 130

6.3 Temperature dependent thermal resistance of length varying vertically aligned carbon nanotubes $\ldots \ldots \ldots \ldots . \ldots . \ldots . \ldots 136$

$6.3 .1 \quad$ Sample preparation and description . . . . . . . . . . . 137

6.3 .2 Thermal resistance measurement and models . . . . . . . . 139

6.3 .3 Low temperature thermal transport . . . . . . . . . . . . . 144

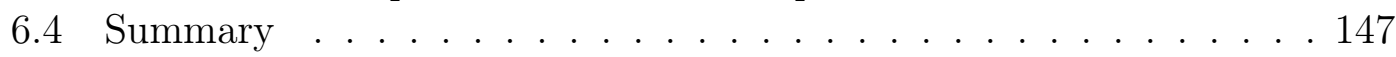

7 Thermoelectric phenomena in graphene constrictions 148

7.1 Introduction . . . . . . . . . . . . . . . . . . 148

7.2 Microheater thermometry . . . . . . . . . . . . . . . . . . . . 149

7.2 .1 Temperature mapping of the junction heater . . . . . . . 149

7.2 .2 Null-point measurement of heater excess temperature . . . 152

$7.2 .3 \quad$ Low temperature thermometry $\ldots \ldots \ldots \ldots$. . . . . . 154

7.3 Exploring thermoelectric effects in graphene constrictions . . . . . 157

7.3 .1 Thermometry measurement principles. . . . . . . . . . . 158

7.3 .2 Scanning Thermal Gate Microscopy (SThGM) . . . . . . . 158 
7.3 .3 Main results . . . . . . . . . . . . . . . . . . . . . . . 159

7.4 Summary . . . . . . . . . . . . . . . . . . . . 165

8 Conclusion and perspectives 166

8.1 Major achievements of this thesis . . . . . . . . . . . . 166

8.2 Perspectives and challenges for nanoscale thermal measurements . . 168

\begin{tabular}{lr}
\hline A Appendices & $\mathbf{1 7 0}$
\end{tabular}

A.1 Estimation of the contact radius . . . . . . . . . . . . . . . . . 170

A.2 Conical tip temperature distribution model . . . . . . . . . . . . . . 171

A.3 Description of experimental setups . . . . . . . . . . . . . . . . . . 173

A.3.1 General SThM setup components and uses . . . . . . . . . . 173

A.3.2 SThM box . . . . . . . . . . . . . . . . 175

A.3.3 Laser management . . . . . . . . . . . . . . . . . . . . . . . 178

A.4 Python code for approach-retract curve analysis . . . . . . . . . . . 179

\begin{tabular}{lr}
\hline References & 184
\end{tabular} 


\section{List of Figures}

2.1 Effect of nanostructuring on thermal transport: thermal conduc-

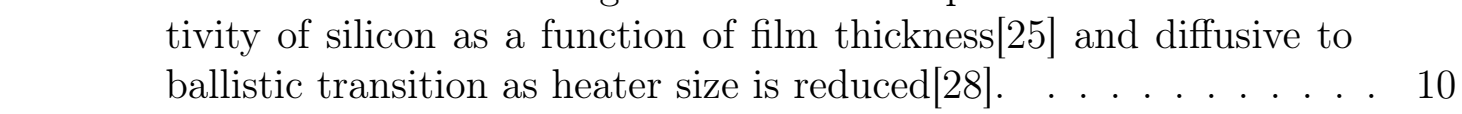

2.2 Schematic tip-sample forces as the probe is approached (blue) to and retracted (red) from the sample. When the tip is in contact with the sample, it obeys to Hooke's law. . . . . . . . . . . . . . . . 14

2.3 Schematic of the UFM operation principles. The sample is oscillating vertically at ultrasonic frequencies by a piezotransducer and the response is measured in the laser deflection signal. . . . . . . . . . . 15

2.4 Standard SThM components using the AFM force feedback loop for positioning and a thermal control unit, usually composed of a Wheatstone bridge, for measuring the thermal probe electrical resistance|8|. . . . . . . . . . . . . . . . . . . 16

2.5 (a) SEM image of the KNT probe 8$]$ with the Pd film joining at the tip end with an apex radius of $50 \mathrm{~nm}$ and (b) Burnt KNT probe after excessive current was applied to the Pd film. . . . . . . . . . 18

2.6 SEM of the doped silicon probe with high doped, low electrical resistance, Si regions and a low doped, high electrical resistance, Si implant used as a heater. . . . . . . . . . . . . . . 19

2.7 Typical Wheatstone bridge for a SThM probe. $R_{a}$ and $R_{b}$ are fixed resistors and $R_{e}$ is variable to balance the probe resistance. The bridge is biased by $V_{i n}$ and the bridge output is the difference between $A$ and B. . . . . . . . . . . . . . . . . . . . . . . . . . . 21

2.8 Schematic of the probe-sample system. Some heat $Q$ is supplied at the sensor which is at $T_{\text {sensor }}$. Sample and microscope are at $T_{\text {sample }}$ and $T_{m}$, respectively. Heat can flow through the cantilever thermal resistancce $R_{p}$ and through the tip-sample thermal resistance $R_{t s}$. . 22

2.9 Null-point method principles $\mid 68]$. Two measurements are performed one just before contact and one in contact. This allows to eliminate the parasitic air heat transfer, $Q_{\text {air }}$, contribution and measure sample-tip heat transfer, $Q_{t s} . \ldots \ldots \ldots \ldots$

2.10 Immersion SThM setup design| $88 \mid$. The liquid forms a meniscus between the liuquid holder and a glass slide above the SThM tip. . 25

2.11 Schematics of the heat transfer mechanisms in the tip-sample system at different lenght scales|8|. The main components are diffusive and ballistic conduction through the surrounding gas, conduction through mechanical contact and through the water meniscus. . . . . 26 
2.12 Model of a heating spot of radius $a$ on a half-plane coating of thickness $t$ on a substrate with boundary conditions|102]. Note that the system has cylindrical symmetry. $\ldots \ldots \ldots \ldots 33$

2.13 Transformation from an orthotropic system to effective isotropic properties $|107|$ and multilayers fluxtube model|108|. . . . . . . . . . 35

3.1 Finite Element Simulation of (a) the self-heated probe and (b) the sample-heated probe with the same temperature of $65 \mathrm{~K}$ (as represented by the color scale). (c) Schematic representation of the probe and the main sources of uncertainty. . . . . . . . . . . . . . 40

3.2 Thermal resistance network with spatially distributed heater and measured probe temperature as a function of tip-sample surface distance. Sample, microscope, and surrounding air heat baths are at temperatures $T_{S}, T_{M}$, and $T_{a i r}$, respectively. Heat produced $Q$ in the heater at the position $l$ is exchanged through 3 channels: $Q_{B}$ through the cantilever resistance $R_{B}$, Qair through the surrounding air resistance $R_{a} i r$, and $Q_{S}$ through the air gap proximity resistance $R_{p}$ and through the contact resistance $R_{x}$. The average heater temperature $\hat{T}_{h}$ is obtained by averaging the temperature at each position $l$ between the tip apex temperature $T_{t}$ and the temperature at the cantilever junction $T_{B} \ldots \ldots \ldots \ldots$. . . . . . . . . . . . . . 43

3.3 Ambient SThM setup with Peltier modules and sensors for temperature control. . . . . . . . . . . . . . . . 46

3.4 Approach (blue) - Retraction (red) SThM curves acquired with PdRP in ambient (a) and vacuum (b) conditions on a $\mathrm{SiO}_{2} / \mathrm{Si}$ sample. Inset: Thermal response $(d V / V)$ obtained when aligning the laser at 3 positions $(\mathrm{A}, \mathrm{B}, \mathrm{C})$ and compared to response without laser. 47

3.5 Approach (blue) - Retraction (red) force curve (top) andSThM curve (bottom) acquired simultaneously with PdRP in ambient conditions on a aluminium oxide sample. Note the periodic noise observed in both signals due to laser interferences. . . . . . . . . . . . 48

3.6 (a) Temperature of the microscope (red filled circles) and sample (yellow open cirles) vs time and (b) the corresponding measured SThM signal (excess probe temperature) (V) for tip out of contact (blue open circles) and in-contact (red filled circles) during consecutive point contacts on Si sample vs time. (c) Thermal response $(d V / V)$ acquired from consecutive point contacts at controlled (constant sample-microscope temperature) (green filled circles) and noncontrolled (red open circles) sample temperature. $\quad$. . . . . . . . . . 49

3.7 (a) Heater temperature distribution for the self-heated case (triangles) and heating through the tip (circles) as obtained from finite element modelling (open circles and triangles) and analytical model (filled circles and triangles). (b) Values of computed temperature rise obtained for the same electrical reading: color of the heater at the edge of the probe schematically represents temperature distribution in the probe. The two temperatures are often considered to be the same. . . . . . . . . . . . . . . . . 53 
3.8 Modelled temperature rise dependence on the electrical resistance for the average heater temperature (dotted blue) and the tip temperature (green line). . . . . . . . . . . . . . . . . . . . 54

3.9 Experimental (solid circles) and model (solid lines) results for the thermal response $d V / V$ as a function of sample temperature in vacuum (left) and in air (right) for three average heater excess temeprature $(5 \mathrm{~K}, 8 \mathrm{~K}$ and $17 \mathrm{~K})$. Dashed lines in panel (b) are a guide for

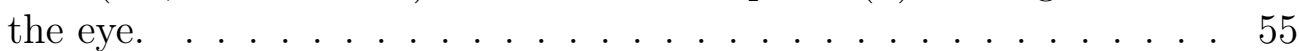

3.10 (a) Vacuum SThM chamber with cooling capabilities. (b) cryoSThM sample holder thermally insulated from the surrounding via Teflon

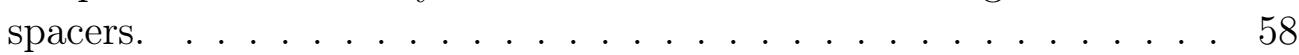

3.11 Schematic diagram of the cryoSThM system in vacuum. The sample holder is connected to a Dewar via a copper braid. The probe heater is at the temperature $T_{h}$ thermally connected to the microscope at temperature $T_{M}$ through the probe thermal resistance $R_{p}$ and to the sample at temperature $T_{S}$ through $R_{X}$ that is made of the conical tip thermal resistance $R_{\text {tip }}$; the interface resistance $R_{i n t}$ and the spreading resistance of the sample $R_{s p r} . \ldots \ldots \ldots \ldots$. . . . . . 60

3.12 Measured thermal resistance in contact with $\mathrm{SiO}_{2}$ (left) and $\mathrm{Si}$ (right) at various sample temperatures with two probe heater temperatures at $350 \mathrm{~K}$ (blue) and 450K (red). The measurement on silicon oxide can be modelled by a standard diffusive model (green dotted line) but the silicon results require more interpretation. For silicon oxide, the two heater temperatures do not coincide highlighting that the hot probe heats the sample and therefore changes the thermal conductivity locally. For silicon, this is not observed as silicon as a much higher thermal conductivity. . . . . . . . . . . . . 63

3.13 SThM response $d V / V$ measured in vacuum as a function of sample air exposition time for two heat temperatures (black and red dots). As time increases, the response diminishes which transposes in an increase of the measured thermal resistance. . . . . . . . . . . 66

3.14 Temperature distribution in a conical tip using a diffusive model for two sample temperatures, $300 \mathrm{~K}$ (blue) and $150 \mathrm{~K}$ (green). Most of the temperature drop within the tip occurs within the first $100 \mathrm{~nm} . \quad 67$

3.15 Custom under-liquid SThM probe holder. A glass disk is placed above the probe in order to create a meniscus covering the probe and the sample allowing stable refraction of the laser beam. . . . . 70

3.16 Schematic representation of the liquid Teflon holder containing the piezotransducer and the sample. . . . . . . . . . . . . 71

3.17 Topographical, SThM (thermal) and UFM (nanomechanical) responses with UFM off and on, in ambient and dodecane liquid environments. All channels are captured simultaneously using a KNT probe operating in active mode. The sample is an exfoliated multi-layer graphene flake on a $\mathrm{SiO}_{2}$ substrate. . . . . . . . . . . . 72

3.18 SThM (plain lines) and UFM spectroscopic (dotted lines) responses as a function of normal force during probe approach to $\mathrm{SiO}_{2}$ substrate in dodecane immersion for various oscillation amplitude. The quasi non-contact regime is signified by the orange shaded region. . 73 
4.1 SThM response $d V / V$ as a function of bulk sample thermal conductivity for metallic (red) and non-metallic (black) samples measured in ambient air conditions. Even within one type of sample, it is difficult to extract a link between thermal conductivity and SThM

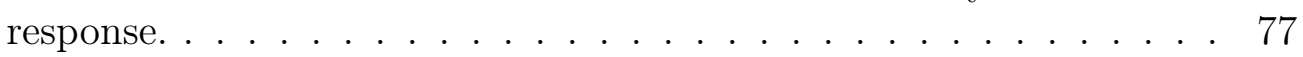

4.2 Optical microscope image of the silicon oxide steps. Each step is around $10 \times 10 \mu \mathrm{m}^{2}$ and its thickness indicated in $\mathrm{nm} . \quad \ldots . . .79$

4.3 Oxide steps thermal resistance measured and the fitted curve using the spreading resistance model with the following parameters: $R_{\text {int }}=2.6 \times 10^{6} \mathrm{KW}^{-1}, a=65.5 \mathrm{~nm}, k=1.0 \mathrm{Wm}^{-1} \mathrm{~K}^{-1}$ and $r_{\text {int }}=4.91 \times 10^{-8} \mathrm{~m}^{2} \mathrm{KW}^{-1} . \ldots \ldots \ldots \ldots \ldots$. . . . . . . 81

4.4 Aluminium oxide layers thermal resistance measured and the fitted curve using the spreading resistance model with the following parameters: $R_{\text {int }}=2.1 \times 10^{6} \mathrm{KW}^{-1}, a=130 \mathrm{~nm}, k=1.81 \mathrm{Wm}^{-1} \mathrm{~K}^{-1}$ and $4.95 \times 10^{-8} \mathrm{~m}^{2} \mathrm{KW}^{-1} . \ldots \ldots \ldots \ldots . \ldots \ldots$

4.5 (a) Schematic illustration of the fabrication process. SEM images of (b) the fabricated microphase separated BCP film on Si substrate and the BCP templates with nominal Cr coatings of (c) $1 \mathrm{~nm},(\mathrm{~d})$ $2 \mathrm{~nm}$ and (e) $5 \mathrm{~nm}$. The darker regions are the PEO domains that were degraded, and the lighter region the PS matrix. SEM images of (f) the fabricated BCP template after the solvent annealing showing the modified morphology and the BCP templates with (g) $2 \mathrm{~nm}$ and (h) $5 \mathrm{~nm}$ Cr layers $\ldots \ldots \ldots \ldots \ldots$. . . . . . . . . . . . . . . . 84

4.6 (a),(c) Topography and (b),(d) thermal resistance images of the nominal BCP templates. Scale bars are $100 \mathrm{~nm}$. PS and PEO regions are indcated by arrows. . . . . . . . . . . . . 85

4.7 (a),(c) Topography and (b),(d) thermal images of the BCP template with $1 \mathrm{~nm}$ Cr layer. Topography and thermal images of the modified BCP templates with 2 (e),(g) and (f),(h) $5 \mathrm{~nm}$ Cr layers, respectively. 87

4.8 High resolution (a) topography and (b) thermal images of the modified BCP template in vacuum environment. (c) Topography and (d) voltage signal profiles along the dashed lines in the images.. . . 90

4.9 Total tip-sample thermal resistance variations as a function of the Cr thickness (black stars) and the two layers on substrate analytical model. The inset graph shows the percentage of the thermal resistance variations between PS and PEO in the BCP films with the pillar (blue spheres) and lamellae (green) morphology. . . . . . 92

5.1 (a) Schematic beam-exit cross-section polishing (BEXP) principles. Ar ions are impinging the sample surface at shallow angle $\left(\sim 5^{\circ}\right)$ creating a SPM friendly surface. (b) Thermal resistance as a function of height starting from the first SiGe layer. Inset: 3D topography overlaid with SThM response. Arrow indicates the direction of the average thermal resistance profile. . . . . . . . . . . . . . 98

5.2 Thermal spreading resistance computed for a constant (red cirlces) and an increasing (blue triangles) layer thermal conductivity and the modelled increasing layer thermal conductivity distribution of SiGe. . . . . . . . . . . . . . . . . . . . . 100 
5.3 (a) Topography image of the BEXP polished $300 \mathrm{~nm}$ silicon oxide sample. The image is flattened on the oxide area. (b) Total thermal resistance map of the $300 \mathrm{~nm}$ silicon oxide on silicon. (c) Average profile along the green arrow direction in (b) fitted with spreading resistance model. Inset is a schematic view of the SThM scanning along the polished sample with increasing thickness. . . . . . . . . . 101

5.4 Comparison of air and vacuum measurements of the same sample. Top: thermal maps of the same sample in air and vacuum. Bottom: SThM profiles normalized to the substrate and top surface measurement . . . . . . . . . . . . . . . 103

5.5 Thermal maps and resistance profiles for the $60 \mathrm{~nm}$ spin-on carbon (left) and $10 \mathrm{~nm}$ spin-on glass samples (right). The fitted data is shown in red. . . . . . . . . . . . . . . . . . . . 105

5.6 Thermal resistance as a function of height for as-grown (blue) and annealed (green) samples. Different slopes of the GeSn region are indicated and are possibly due to different crystal quality in this

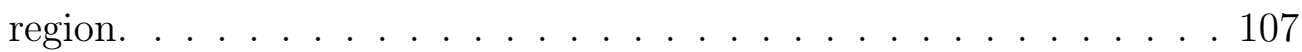

5.7 Left: Finite Element model geometry with several steps mimicking the cross-sectioned surface. Right: thermal conductivity profile across the model steps . . . . . . . . . . . . . . . . . . 108

5.8 Polished $\mathrm{Si}_{1-x} \mathrm{Ge}_{x}$ on silicon sample. Modelled and measured thermal resistance as a function of height and thus increasing Ge composition are compared. . . . . . . . . . . . . . . . . . . 109

5.9 Images of the full interface polished with BEXP. (a) Large view of whole layers, from left to right: capping oxide, polymer, CNTs, seed layer, silicon, scale bar is $50 \mu \mathrm{m}$. (b) UFM image detailing the CNTs-polymer mixing, scale bar is $2 \mu \mathrm{m}$. (c) Detail of the seed layer-CNTs interface, scale bar is $2 \mu \mathrm{m}$. (d) Detail of CNTs region highlighting different CNTs orientation, scale bar is $5 \mu \mathrm{m}$. (e) Detail of CNT bundles. Scale bar is $500 \mathrm{~nm}$. . . . . . . . . . . . . . . 112

5.10 3D topography overlaid with UFM signal. . . . . . . . . . . . . . . 113

5.11 Perpendicular BEXP schematic, not to scale. The sample is mounted on its edge and the polished side is accessible with the SThM probe. 114

5.12 SEM image of the full TIM as obtained from perpendicular nanocross-sectioning. The scale bar is $2 \mu \mathrm{m}$. The dip between the polymer and copper is observed. . . . . . . . . . . . . . . . . 115

5.13 Topography (left) and SThM (right) maps of the BEXP perpendicularly polished sample. . . . . . . . . . . . . . 116

5.14 Optical microscope image of the TIM sample prepared for TEM imaging. . . . . . . . . . . . . . . . . . . . . 117

5.15 Topography (left) and SThM (right) maps and profile of the TEMlike prepared TIM sample. . . . . . . . . . . . . . . . . . . 118

5.16 (a) Schematic of the FIB section sample experiment. While applying a temperature gradient on both sides, the temperature distribution is measured along the TIM. (b) SEM image of the FIB section. . . . . . . . . . . . . . . . . . . 119

5.17 TIM temperature distribution setup and voltage bias applied on the heating resistor. . . . . . . . . . . . . . . 120 
5.18 Topography (top left) and SThM (top right) temperature maps of the FIB sectioned sample when the probe is in- and out-of-contact with the sample. Schematic applied voltage on the resistor to create a temperature gradient (bottem left). Resulting SThM map (bottom right) obtained by substracting the non-contact signal from the in-contact one. . . . . . . . . . . . . . . . . 121

6.1 Optical microscope image of the Franckeite flake with various numbers of layer and franckeite unit cell structure where $\mathrm{M}$ can be $\mathrm{Pb}$, $\mathrm{Sn}$ or Sb, N is either $\mathrm{Sn}$ or Fe and $\mathrm{X}$ is S. . . . . . . . . . . . . . . . 126

6.2 Topography (left) and SThM (right) maps of an exfoliated franckeite flake. Several thickness can be observed with different thermal response. As the franckeite thickness increases, its thermal resistance increases as well. This behaviour is the signature of a lower thermal conductivity material than the silicon oxide substrate. . . . 127

6.3 Thermal resistance received from the SThM meaurements as a function of franckeite thickness for temperatures from 156 to $301 \mathrm{~K}$. The experimental data is plotted with symbols while the spreading resistance model of Eq. 4.4 is shown in dashed lines. . . . . . . . . . . . 129

6.4 Interface thermal resistance $r_{\text {int }}$ and thermal conductivity of franck\begin{tabular}{|c|c|c|}
\hline eite as a function of temperature as obtained from the fitting of Eq. \\
\hline
\end{tabular} 4.4 on the data shown in Fig. 6.3. . . . . . . . . . . . . . . . 130

6.5 Optical microscope image of the $\mathrm{MoS}_{2}$ on graphene heterostructure. 131

6.6 Topography and SThM images of $\mathrm{MoS}_{2}$ on graphene heterostructure obtained at $220 \mathrm{~K}$. The graphene has a lower resistance than the $\mathrm{MoS}_{2}$ and it appears that graphene lower the resistance of the $\mathrm{MoS}_{2}$ when it is below. Scale bar is $500 \mathrm{~nm} . \ldots \ldots \ldots . \ldots 132$

6.7 Thermal resistance as a function of sample temperature for the different areas of the sample. A general increase of the thermal resistance is observed for all regions. . . . . . . . . . . . . . . . 133

6.8 Measured interface thermal resistance as a function of sample tem-

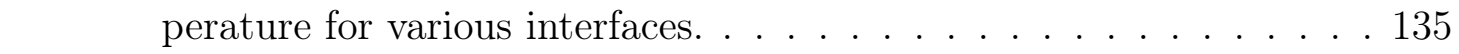

6.9 (a) SEM imaging of the nanotubes bundles with increasing height, scale bar is $1 \mu \mathrm{m}$. (b) Magnified SEM image of the CNT bundles.

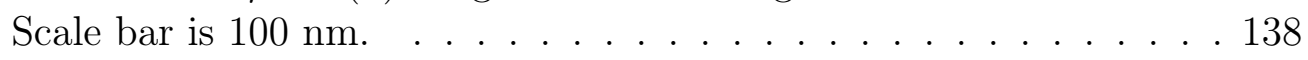

6.10 (a) Top view SEM image showing around $28 \pm 2 \%$ coverage of CNTs. Scale bar is $200 \mathrm{~nm}$. (b)-(c) Topography (left) and SThM (right) maps of the nanotubes bundles. Scale bars are $100 \mathrm{~nm}(\mathrm{~b})$ and 50 $n m(c) . \ldots \ldots \ldots \ldots \ldots \ldots \ldots$

6.11 (a) Thermal resistance measured as a function of nanotubes height and fitting models applied. Inset shows the thermal resistances included between the sensor and the heat sink. SThM (b) and UFM (c) maps of the silicon-nanotubes interface, scale bars are $1 \mu \mathrm{m} .140$

6.12 Nanotubes bundles thermal resistance as a function of their height for low temperature $(\sim 200 \mathrm{~K})$ and room temperature $(\sim 300 \mathrm{~K})$. Inset: bundle thermal conductivity assuming 1D transport for both temperatures. Dashed lines are the ballistic limits. . . . . . . . . . . 146 
7.1 SThM temperature maps for 4 different bias voltages on the junction \begin{tabular}{|l|}
\hline heater. From left to right: $1.5 \mathrm{~V}, 1.3 \mathrm{~V}, 1.06 \mathrm{~V}$ and $0.75 \mathrm{~V}$. The \\
\hline \hline temperature scale on the images is the excess temperature with \\
\hline \hline
\end{tabular} respect to the surrounding and is a guide value. . . . . . . . . . . . 150

$7.2 \quad$ SThM traces along the junction direction for various heater voltages. The temperature gradient was estimated in the graphene junction

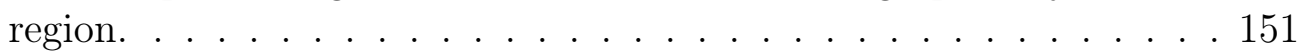

7.3 SThM images and line traces of $14 \mathrm{~Hz}$ and $70 \mathrm{~Hz}$ square pulses applied to the heater. For $70 \mathrm{~Hz}$, thermal equilibrium is not reached as seen in the SThM signal. . . . . . . . . . . . . . . . . 152

7.4 Left: example of a typical SThM scan showing approach (blue) and retract (red) SThM signal. Right: temperature jump as a function of probe excess temperature for two heater temperatures. The green arrow highlights the temperature difference sensed by the probe between heated and non-heated sample. . . . . . . . . . . . . 153

7.5 Finite element simulation of the SThM probe temperature. Weighted tip heater temperature is around $307 \mathrm{~K}$ that is around $14 \mathrm{~K}$ above the ambient $\mathrm{T}$, whereas the probe tip temperature is around $323 \mathrm{~K}$ that is $30 \mathrm{~K}$ above the ambient T. . . . . . . . . . . . . . . . . 154

7.6 (a) Experimental setup with $T$ controlled Peltier stage. (b) Experimental measurement and (c) explanation of the principle of additive approach to the calibration of the absolute rise of the sample temperature due to the heater. A rectangular heating voltage on the heater results in a change of the SThM signal $\Delta V_{h}$, the stage temperature was then increased by $\Delta T_{0}=5 \mathrm{~K}$ leading to a shift of SThM response by $\Delta V_{s t} . \ldots \ldots \ldots \ldots \ldots$. . . . . . . . . . . 155

7.7 Topographical (a) and thermal (b) images of the active heater with the corresponding profiles (c, d) along the lines shown in the images. 157

7.8 Topography of a few layers graphene bowtie constriction. . . . . . . 160

7.9 Peltier and Joule heating images of a few layers graphene bowtie constriction. Scale bar is $500 \mathrm{~nm}$. . . . . . . . . . . . . . 161

7.10 Thermovoltage map of a few layers graphene bowtie constriction. Scale bar is $500 \mathrm{~nm} . \ldots \ldots \ldots \ldots 2 \ldots \ldots \ldots$

7.11 Topography and Joule heating images of a few layers graphene bowtie constriction. . . . . . . . . . . . . . . 163

7.12 Peltier, Joule heating and thermovoltage images of the long ribbon geometry. Scale bar is $200 \mathrm{~nm}$. . . . . . . . . . . . . . 163

7.13 Peltier, Joule heating and thermovoltage images of the mutliple islands geometry. Scale bar is $500 \mathrm{~nm}$. . . . . . . . . . . . . . 164

A.1 Doped silicon tip SEM image used for estimation of the contact area. Scale bar is $200 \mathrm{~nm} \ldots \ldots \ldots$. . . . . . . . . . . 170

A.2 Comparison of topography and thermal signal to estimate the tip contact radius from $|113| . \ldots \ldots \ldots \ldots$. . . . . . . . . . . . . . . .

A.3 Schematic of the conical tip. . . . . . . . . . . . . . . . . . . . 172

A.4 Principals SThM components. . . . . . . . . . . . . . . . . . . . . 174

A.5 SThM box with precise amplification. . . . . . . . . . . . . . . . . . 175

A.6 $\quad$ SThM box circuit with preamplifier. . . . . . . . . . . . . . . . . . . 177

A.7 $\quad$ Laser blocking nanopaddle . . . . . . . . . . . . . . . . . . . . . . . 178 
A.8 HF etched and non-etched silicon wafer with water droplet showing different contact angle. . . . . . . . . . . . . . . . . . . 182 


\section{List of Tables}

3.1 Measured nominal values of the model parameters. . . . . . . . . . 51

4.1 Parameters of the 2 layers spreading resistance model. . . . . . . . . 89

5.1 parameters obtained by fitting equation 4.4 to experimental data. For silicon oxide, literature values are used to obtain $a$ and $R_{\text {int }}$. Bold values are assumed from literature and italic value is derived from the $\mathrm{SiO}_{2}$ calibration. . . . . . . . . . . . . . . . . . 105

6.1 Thermal conductivity obtained with the two models applied. . . . . 143

7.1 Temperature gradient for the 4 bias voltages as obtained by linear fit and slope measurement. . . . . . . . . . . . . . . . . 151 


\section{Glossary and Abbreviations}

AFM Atomic force microscopy

ALD Atomic layer deposition

BCP Bloc copolymer

BEXP Beam-exit cross-sectional polishing

CNT Carbon nanotube

cryoSThM SThM performed at cryo temperatures.

FEA Finite element analysis.

FMM Force modulation microscopy.

FIB Focused ion beam.

iSThM immersion SThM.

MBE Molecular beam epitaxy.

MFP Mean free path.

PEO Polyethylene oxide.

PS Polystyrene.

SEM Scanning electron microscopy.

SPM Scanning probe microscopy.

SThM Scanning thermal microscopy.

TEM Transmission electron microscopy.

TIM Thermal interface material

SThM response Quantity measured during an SThM experiment.

UFM Ultrasonic force microscopy.

2D materials Materials composed of a single atomic layer. 


\title{
List of symbols
}

\author{
$T$ Temperature
}

$k$ Thermal conductivity with unit $\mathrm{Wm}^{-1} \mathrm{k}^{-1}$

$Q$ Heat transferred with unit W

$\Lambda$ Phonon mean free path

$a$ Contact radius at the SThM tip (unless stated otherwise)

$K n$ Knudsen number, often computed as $\Lambda / a$

$R$ Thermal resistance (unless stated otherwise) with unit $\mathrm{KW}^{-1}$

$d V / V$ SThM response, relative jump in SThM signal between signal just before contact and signal in contact

$t$ Thickness

$r_{\text {int }}$ Interface thermal resistance with unit $\mathrm{m}^{2} \mathrm{KW}^{-1}$

$S$ Seebeck coefficient

$\omega$ Frequency 


\section{Outline and motivations}

Very few methods are available to investigate nanoscale heat transport. Moreover these methods often lack reliability, spatial resolution or both. Even simple geometries such as thin films on substrate present many challenges to traditional techniques for thermal conductivity measurements. As the need to study nanothermal properties creates challenges on both scientific and technological sides, there is a pressing demand for a suitable tool able to address these questions. While Scanning Thermal Microscopy (SThM) was first developed at the microscale, nanoprobes are now able to measure local thermal properties down to few nanometers. However, many open questions remain on both the interpretation of SThM measurements and their ability to quantify nanomaterials properties.

Achieving stable and reproducible results is crucial in any method. The number of influencing parameters in the SThM nanothermal system create many uncertainties and renders experiments difficult to compare. Even though qualitative results are easily obtained, as with many other scanning probe microscopes, there is a need for quantitative information which SThM struggles to provide. Opportunities are almost limitless in research and developments for SThM applications but these hurdles create barriers to its implementation.

The main goal of this thesis is to create and demonstrate a comprehensive framework in approaching SThM measurements combining both analytical and experimental developments of SThM systems. This is realised through three major achievements. The first combines SThM with a nano-cross-sectioning tool allowing measurements of materials nanothermal transport in 3 dimensions. The second creates a temperature controlled cell for stable and reproducible measurements. The last develops a novel measurement setup enabling to study nanoth- 
ermal transport at cryogenic temperatures. The combination of these approaches achieves quantitative measurements of various nanostructured materials.

While chapter 2 reviews the literature regarding the main concepts used in this thesis, namely nanothermal transport, SThM principles and thermal resistance modelling, chapter 3 exposes comprehensive frameworks essential to SThM quantitative measurements. We then apply these frameworks in chapter 4 and extract thermal properties quantitatively from measurements of standard materials and novel nanomaterials. Chapter 5 further develops ideas laid down in chapter 4 and adds a third dimension to the map by $3 \mathrm{D}$ probing material properties. Using concepts and systems developed before, we present in chapter 6 quantitative SThM measurements of low dimensional materials such as carbon nanotubes and 2D materials. Finally, chapter 7 takes another angle and looks at thermometry of graphene thermoelectric devices. This last chapter opens new areas for SThM exploration of novel physical phenomena. 


\section{Background review}

\subsection{Introduction}

The quest of nanotechnology can be divided in two essential parts: the continuing down sizing of the systems of interests on one hand, and the hope of discovery of novel properties appearing at the nanoscale on the other hand. Both trends coexisted since the early times of nanoscale research with, for example, the invention of Scanning Tunneling Microscopy (STM)[1], which enables materials study and manipulation at the atomic level, and the discovery of electronic properties of silicon paving the way for our contemporary computer era.

Since then, many techniques were developed both for the fabrication and the characterization of material and devices properties. They highlighted the enormous potential of materials when prepared in a particular configuration. A major branch of those techniques is formed by the scanning probe microscopy (SPM). Atomic force microscopy (AFM)[2], Scanning Thermal Microscopy (SThM) 3] and Ultrasonic Force Microscopy (UFM) [4] will be used in our work.

Although, as confinement and size reduction of materials and devices opened numerous possibilities and created ever smaller or faster computers, limitations were also discovered. Heat management, electrical conductivity or band gap limits are examples of issues facing the future development of electronics. This leads to the search for new materials, such as two-dimensional (2D) materials, which have the advantages of being atomically thin and robust, and promises of tunable properties arising from the diversity of $2 \mathrm{D}$ materials present in nature or that can be synthesised. 
Major issues stall the development of nanoscale systems due to a lack of understanding of thermal phenomena. Hot spots creation in transistors arrays [5], or reduction of the thermal conductance in nanostructured materials $\mid 6]$ are examples that limit further developments and improvements of device efficiency. Theoretical models are often limited as the ballistic nature of thermal transport needs to be accounted for and the diffusive approximation is not verified|7].

To address these challenges, a tool was developed based on the generic AFM principles $[8]$. The Scanning Thermal Microscope provided the ability to resolve thermal properties with high sensitivity at high spatial resolution. In many cases, this high spatial resolution is needed as nanostructures are below resolution (the diffraction limit) of standard optical techniques 8. Also, this nanostructuring of matter creates new phenomena difficult to assess with other techniques.

This literature review can be divided in three interlinked parts. The first part focuses on heat transfer at the nanoscale and how it differs from the macroscale. The second one deals with Scanning Thermal Microscopy and explains SThM measurements. Finally, we review two approaches to thermal resistance modelling that will be used in this thesis: Finite Element Analysis (FEA) and analytical expressions of the thermal spreading resistance.

\subsubsection{Existing techniques and limitations}

In order to gain insights about the heat transport properties of a sample, several techniques are available and are usually based on a temperature depend phenomena. Two categories can be generally described: electrical and optical methods. We review some most common techniques and expose their limitations.

Electrical methods Using a microfabricated sensor, such as gold lines or Pt resistors, precise measurements can be performed on a sample at any time. The sensor can also be buried within the architecture, if needed, such as in packaged devices. Fabrication processes allow sensors to be very localized or, if necessary, global. However, detailed pictures of the temperature cannot be obtained and in the case 
that heat sources are smaller than the sensor dimensions, it does not provide a clear understanding of the temperature distribution of the device. Furthermore, embedding sensors within or on top of a sample requires extra fabrication processes which can be costly, time consuming and often impossible to realise.

Optical methods Optical techniques can provide a more versatile tool for thermometry if only surface and near-surface properties are of interest. Three categories can be drawn|9]: time-domain techniques, frequency-domain techniques and spectroscopy techniques. Time-domain techniques include notably time-domain thermoreflectance (TDTR) and the laser flash method. Using TDTR, Cahill et al. measured interface thermal conductance between dissimilar materials 10 and, more recently, the thermal conductivity of intercalated molybdenum disulfide thin film[11. The laser flash method is usually used for bulk materials 12 as small thicknesses and interfaces are more challenging for this method. However, it can be applied to nanoengineered materials such as thermal interface materials using graphene-polymer composite 13.

Frequency-domain techniques include numerous methods amongst which frequencydomain thermoreflectance (FDTR) which can be easier to implement than its timedomain counter part $[9]$ and photothermal emission method. FDTR was used when Malen et al. [14, 15] investigated phonon mean free path contributions to thermal conductivity in crystalline semiconductors. Using the photothermal method, Chen et al.[16] introduced a microscopy technique based on plasmon generation. Their setup allows high temporal resolution $(10 \mu \mathrm{s})$.

Many other optical techniques were developed and this space is not the place to deeply review each of them. We mention a last one often encountered in the literature: Raman thermography. The basic principle uses the dependence of the scattered photon spectra on the crystal temperature. Balandin et al. used Raman techniques to measure thermal conductivity of $2 \mathrm{D}$ materials 17,18 . One of the drawbacks of using Raman for thermal properties measurements is the need to know the absorbed power in the sample. This often limits its use to suspended 
materials as in this case heat flow can be considered in only two dimensions 19 .

All optical techniques suffer a major hurdle which is the spatial resolution limitation due to diffraction[20]. Optical methods are under the Rayleigh criterion constraint $(\lambda / D$, where $\lambda$ is the wavelength and $D$ the characteristic size of an object to observe) which typically doesn't allow objects smaller than $100 \mathrm{~nm}$ to be resolved. This issue is easily solved when using Scanning Thermal Microscopy as the resolution is typically limited by the size of the solid-solid contact and therefore very fine structures can be imaged. Also, SThM does not require a long fabrication process compared to electrical methods and is suitable for many sample types. As we will show in this thesis, it provides a versatile solution for thermal analysis such as local temperature or thermal conductance. Nevertheless, as is often the case, the best approach is to combine several techniques and correlate their results as for example in Gehring et al.21 where graphene-fullerene thermoelectric nanodevices were investigated both using SThM, electrical and analytical methods.

\subsection{Heat conduction at the nanoscale}

If, somewhere in space, there exists a temperature difference between a lower and higher temperature spots, energy transfer will spontaneously occur. This energy transfer is the subject of heat transfer physics that aspires to describe in detail all the transport processes from this temperature difference. Heat transfer is possible through many mechanisms and media. In macroscopic scales, it is often considered that conduction transports energy through lattice vibrations or electrons in solids. In liquids, it is convection that transports heat through fluid movement. Finally, heat can be transfered by radiation with photons. These phenomena have been widely studied and are well described in textbooks for macroscopic scales 22]. When the system dimensions are reduced, more mechanisms need to be considered. Also, laws governing macroscopic scales are not necessarily valid when reaching microscopic and nanoscopic scales. Near-field radiation can increase the heat transfer by orders of magnitude 23 compared to far-field. In fluids and solids, conduction 
enters different transport regimes and can become ballistic. However, there is no general single limit for the different regimes as they are dependent on materials properties and the temperature. In this section we briefly review how conduction changes at the nanoscale and upon which conditions. We limit this review to heat conduction in solid materials as it is the most relevant for the rest of this study.

\subsubsection{Diffusive transport and its nanoscale limit}

At the macroscale, thermal conduction is governed by Fourier's law which can be written for an homogeneous medium as 24:

$$
\phi=-k \nabla T
$$

where $\phi$ is the heat flux, $k$ the thermal conductivity and $T$ the temperature. For a transient temperature distribution, one can derive the following equation

$$
\nabla(k \nabla T)+\dot{q}=\rho c_{p} \frac{\partial T}{\partial t}
$$

where $\dot{q}$ is the volumetric thermal energy generation rate, $\rho$ is the density and $c_{p}$ the heat capacity. If there is no internal energy conversion and if the thermal conductivity can be assumed to be temperature independent, we obtain at steady state:

$$
\nabla^{2} T=0
$$

which is known as Laplace's equation. With sufficient initial temperature distribution and boundary conditions, it can be solved both analytically for simple cases and numerically in more complex geometries.

These equations are under the assumption of local equilibrium and allow the definition of temperature

$$
T=\left(\frac{\partial U}{\partial S}\right)_{V, N}
$$

as the partial derivative of the system internal energy $U$ with respect to entropy 
$S$ at constant volume $V$ and number of particle $N$. When dealing with small systems, in time or space, Fourier's law is invalidated as non-equilibrium processes are involved. For such systems, the Boltzmann transport equation (BTE) describes transport phenomena at small length scales and time scales. In fact, Fourier's law and macroscopic equations can be derived for the BTE. A detailed analysis of transport equations would be beyond the scope of this work.

Heat and temperature are local quantities accounting for the energy of a small volume. When letting this local energy reach equilibrium, it needs to dissipate through the media. In condensed matter, this is generally realised via two energy carriers, phonons and electrons 22. Phonons are quantized modes of vibrations. Particularly evident in crystalline solids, heat is transferred through atomic vibrations. Atoms oscillate around their equilibrium position in the lattice. As we will see, at the macroscale, phonons have a particle-like behaviour and heat is transfered as a diffusion process involving many scattering events. At micro and nanoscales, phonons behave as waves and can travel ballistically across the media as scattering tends to be diminished. Similarly, in metals, heat conduction is realised mostly with electrons. Phononic conduction also contributes but generally electronic conduction dominates 22. In metals, the electronic thermal conductivity is generally obtained from the Wiedemann-Franz law relating electrical conductivity $\sigma_{e}$ to thermal conductivity $k_{e} \mid 22$

$$
k_{e}=N_{L} \sigma_{e} T=2.442 \times^{-8} \sigma_{e} T
$$

where $N_{L}$ is the Lorenz number, supposed to be constant for metals. However this relation doesn't hold when low temperatures are reached.

When considering nanoscale systems, different mechanisms have to considered compared to large scale objects $[6]$. As the system sizes are reduced, boundaries start to dominate due to carrier collisions with interfaces. If a heat generating device is sufficiently small, it can experience an extra resistance arising from the phonon's density variation in the device. Finally, nanostructuring materials alters 
their phonon spectra. All these fundamental differences play a key role in understanding the temperature distribution in electronic devices and for developing new technologies.

When phonons are treated as quasi-particles, a mean free path (MFP) is associated with the thermal conductivity. In a bulk medium, assuming that all modes carry the same energy (grey medium assumption)[15], we have:

$$
k=\frac{C v \Lambda}{3}
$$

where $C$ is the specific heat per unit volume and $v$ is the speed of sound. In the bulk, $\Lambda$ depends on various intrinsic phenomena such as phonon-phonon scattering and impurity scattering. In a real crystal, phonon dispersion needs to be taken into account and it gives a more realistic MFP as it considers the frequency dependence of the specific heat and the group velocity 22 :

$$
k=\frac{1}{3} \sum_{p} \int C(\omega) v(\omega) \Lambda(\omega) d \omega
$$

A key number for estimating limits to the diffusive approximation is the ratio of the phonon mean free path $\Lambda$ to a characteristic physical length $L$, the Knudsen number: $K n=\Lambda / L$. This length depends on the system and can be the film thickness for a thin film. When $K n<1$, diffusive transport still holds but when $K n>1$ ballistic contributions need to be included, as schematised on Fig. 2.1. Two physical effects that will be observed and discussed in this work affect the Knudsen number: the size effect on the MFP and the nanoscale size of the heat source. In the first case, size reduction effectively increases phonon scattering. This is typically observed as a reduction in thermal conductivity. As more scattering events occur, less phonon modes are available to carry heat away and therefore thermal conductivity follows. Fig. 2.1 a shows the thermal conductivity reduction as the film thickness is reduced[25], a phenomena observed in nanostructured heat sources such as nanodevices. If the device size becomes comparable or smaller than 
the substrate phonon MFP, heat spreading is less efficient. This can lead to device failure and is therefore of great interest in device fabrication. One can think of this effect as a gas flowing through a constriction. Prasher 26, 27] modelled heat conduction through a nanosized constriction with such a model. As the heat source size is similar to the phonon MFP, less modes become populated or it becomes harder to populate various modes and this effectively creates a resistance.
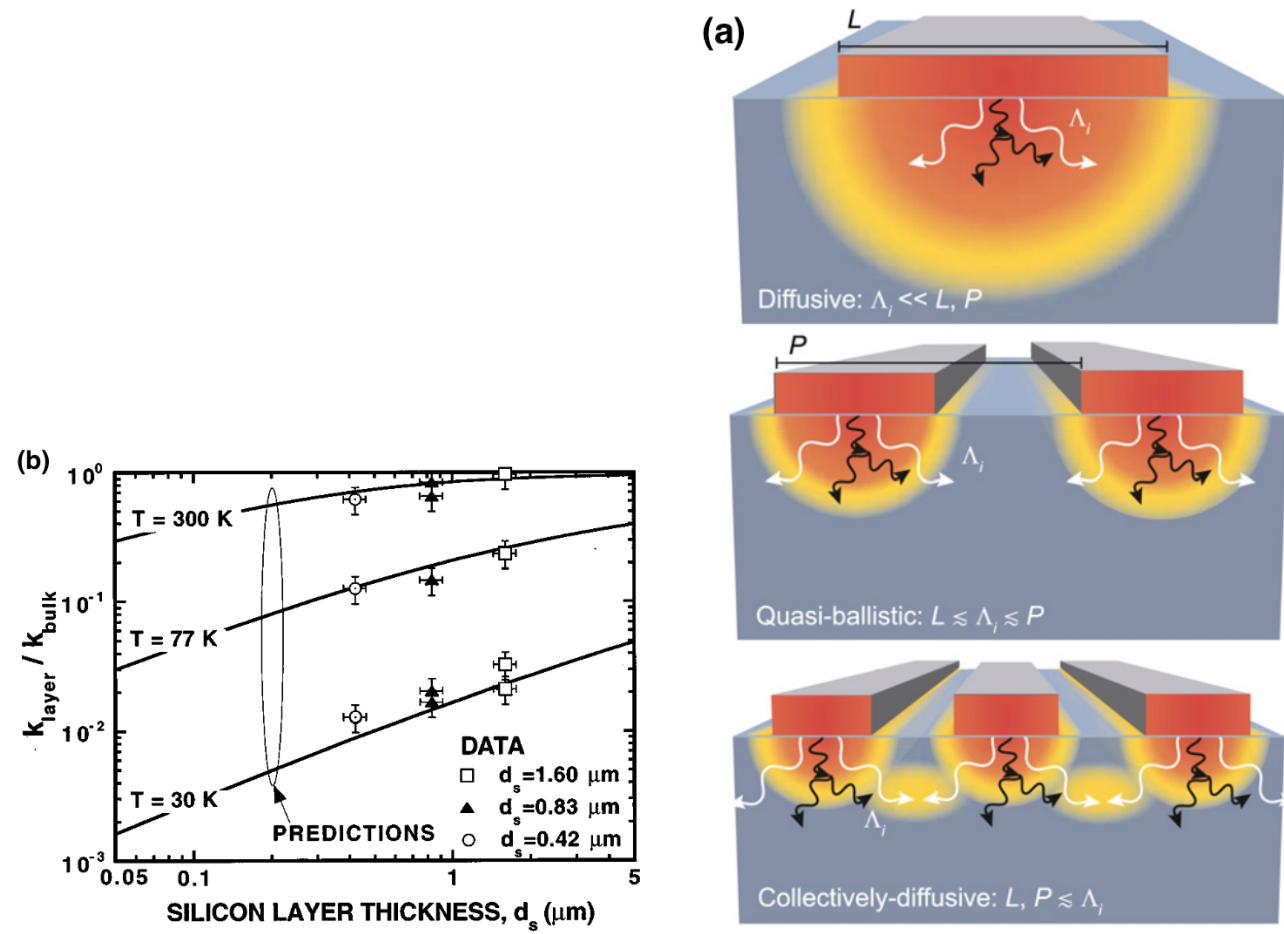

Figure 2.1: Effect of nanostructuring on thermal transport: thermal conductivity of silicon as a function of film thickness 25 and diffusive to ballistic transition as heater size is reduced $[28]$.

Understanding the contribution to conductivity of the various phonons is therefore crucial. For example, in silicon, long MFP $(>1 \mu \mathrm{m})$ contribute mostly to thermal conductivity 15. Therefore, nanostructuring breaks down long MFP phonons while short MFP are unaffected. Exploring the thermal conductivity contribution of the different MFP lengths then helps in understanding the thermal conductivity reduction of nanostructures 29. At the nanoscale, Fourier's law overestimates thermal conduction when the heat source is smaller than the MFP. In this case, the characteristic size to estimate the Knudsen number is the dimension of the heat source. Hoogeboom et al.[28] unveiled a new transport regime using multiple heat 
sources. They showed that when heat sources are closely arranged, they can have a diffusive-like transport as long MFP phonons interact as if they were coming from the same heat source (Fig. 2.1p).

More generally, as explained by Siemens et al.|30| a reasonable correction of Fourier transport is obtained by summing diffusive and ballistic components. In fact, this can be thought as having a geometrical transformation by adding length and MFP $L \rightarrow L+\Lambda$. Similarly, Prasher 27] derived a useful model including both diffusive and ballistic components of a constriction resistance:

$$
R_{c}=\frac{1}{2 k a}\left[1+\frac{8}{3 \pi} K n\right]
$$

where $k$ is the thermal conductivity of the material and $a$ the constriction dimensions.

\subsubsection{Transport at interfaces}

As two media are joined, an interface is created. Interfaces give rise to various phenomena and are typically obstacles for transport, both electrical or thermal. The study of thermal conductance at interfaces began with experiments on superfluidic helium. Kapitza|31] in 1941 measured a thermal resistance between helium and a solid, the Kapitza resistance. Later, models were developed to explain this resistance and it was understood that it would exist between any materials. Precise control of thermal conductivity was achieved by nanostructuring interfaces to act as phonon-scattering barriers 32. Many models and experiments were reported, however no common framework has appeared to commonly describe thermal transport at interfaces. One of the experimental challenges is to create well-controlled experiments as many parameters enter into play. For example, the roughness down to the atomic level is very difficult to know precisely. Also, the crystalline orientation of the two contacting surfaces cannot be controlled with sufficient certainty.

Similarly to optics, where interfaces refract incoming rays, the difference in densities and sound speeds creates a boundary for the propagating heat carriers. 
Two models are generally used to represent an interface between dissimilar materials, the acoustic-mismatch model (AMM) and the diffusive mismatch model (DMM). The AMM assumes that there is no scattering at interfaces and gives a phonon transmission coefficient from a material $A$ to a material $B[33$ :

$$
t_{a b}=\frac{4 Z_{A} Z_{B}}{\left(Z_{A}+Z_{B}\right)^{2}}
$$

where the acoustic impedance is defined as $Z=\rho c$ with $\rho$ and $c$ the density and sound speed, respectively. Therefore, good thermal propagation over an interface between two materials requires the speed of sound in both material to be similar either side of the interface[34]. If the two materials are identical then $t_{a b}=1$ which doest not represent the system correctly. In the DMM, the assumption is that incoming phonons scatter at the interface independently of where they are coming from. The probability of being reflected or transmitted across the interface then depends on the available phonon density of states on the two sides of the interface. Generally, AMM is valid for long wavelength phonons while DMM is correct at short wavelengths 35 .

\subsection{Scanning thermal microscopy (SThM)}

We saw that nanoscale heat transfer presents many challenges for both theory and experiments and therefore its study is key to deepen our understanding of physical systems as well as to solve current issues face in technological applications. A promising tool for investigating nanoscale heat transfer was developed in 1986 even before the invention of the AFM[3]. First thought as a profiler using heat flux to map a surface topography, similarly to a STM, it rapidly attracted interest for its local heating capabilities. We first review in this part the development and principles of Scanning Thermal Microscopy. Then we compare different modes of operations of the SThM set up including different environment conditions. The tip-sample system is then described with the various heat transfer mechanisms 
at stake. Finally, before addressing the challenge of what SThM measures, we review different methods for quantitative nanothermal measurements. However beforehand, we expose the general principle of scanning probe microscopy.

\subsubsection{Scanning probe microscopy}

To overcome the optical tools limits, the diffraction limit, a new tool needed to be developed. This was realised by Binning and Rohrer[1] in 1982 when they invented the Scanning Tunneling Microscope (STM) and in 1986 were awarded the Physics Nobel prize for the invention. The same year, Binning, Quate and Gerber[2] developed the Atomic Force Microscope (AFM). The STM relies on electron tunnelling between a tip and a surface. This tunnelling current is kept constant by varying the tip-sample distance. The sample surface therefore needs to be conducting. The main advantage of AFM is to remove this necessity as instead of tunnelling current, interatomic forces are kept constant as we will explain later.

Since these early inventions, many scanning probe microscopy (SPM) techniques were developed. Each technique is able to probe on the nanoscale a particular property of a system, such as electrostatic forces, magnetic forces, thermal conductance, stiffness, etc. The basic principle of each SPM technique depends on the feedback mechanism it uses. In this thesis, mainly contact mode AFM was used. Contact mode relies on interatomic forces to keep the tip-sample forces constant. Figure 2.2 shows the tip-sample forces as a function of separation distance. When the tip-sample distance becomes very small, the interaction forces and capillary forces overcome the spring resistance of the cantilever and it snaps-in contact. When the probe pulls out, the attraction forces are generally bigger and therefore a higher separation is needed to break contact. 


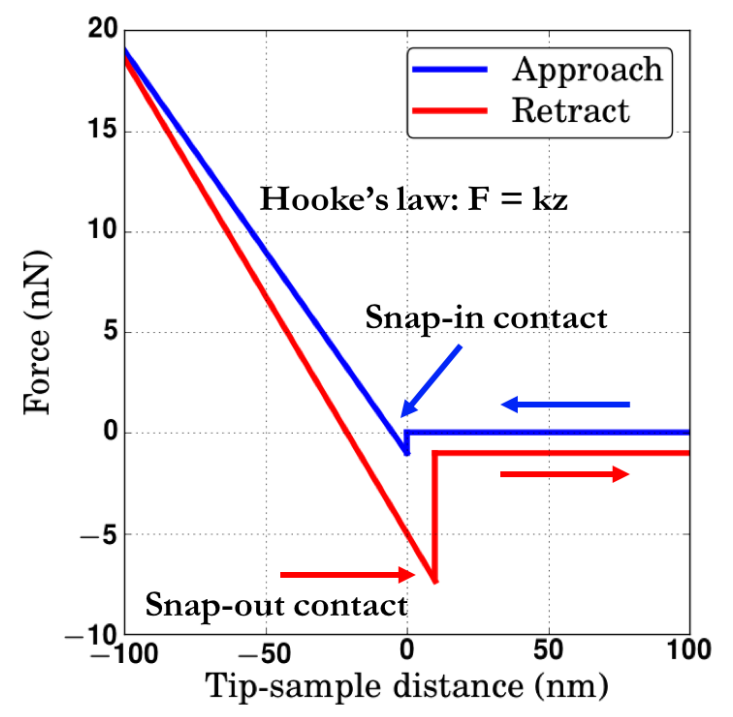

Figure 2.2: Schematic tip-sample forces as the probe is approached (blue) to and retracted (red) from the sample. When the tip is in contact with the sample, it obeys to Hooke's law.

In contact mode, the probe-sample distance is slightly varied to keep the force constant via a feedback loop on the separation. For example, let's assume the probe is moving on the sample surface. If a topographical feature approaches the tip apex, interatomic forces will create first attraction and then repulsion forces. This causes variations on the probe bending and can be observed through the deflection of a laser beam on the back of the cantilever. This deflection is monitored throughout the scanning process. If it changes slightly, the feedback mechanisms will increase or decrease the tip-sample distance to maintain the same deflection signal.

An example of SPM technique resting on the contact mode principle is the Ultrasonic Force Microscopy (UFM) that will be used in the next chapters. UFM allows nanoscale mapping of local material stiffness variations $4,36 \sqrt[38]{38}$. This technique uses the same feedback mechanisms as regular contact mode AFM. However, the sample is oscillated vertically at very high frequencies $(>4 \mathrm{MHz})$ through a piezoeletrical sample holder (see Fig. 2.3p. This creates a vertical displacement from a few angstroms to a few nanometers. The cantilever cannot follow such high frequency modulation and therefore remains stationary slightly above the sample 
surface. As such, UFM is a quasi-non contact technique and it was shown that ultrasonic excitation induces a high reduction in friction forces 39.

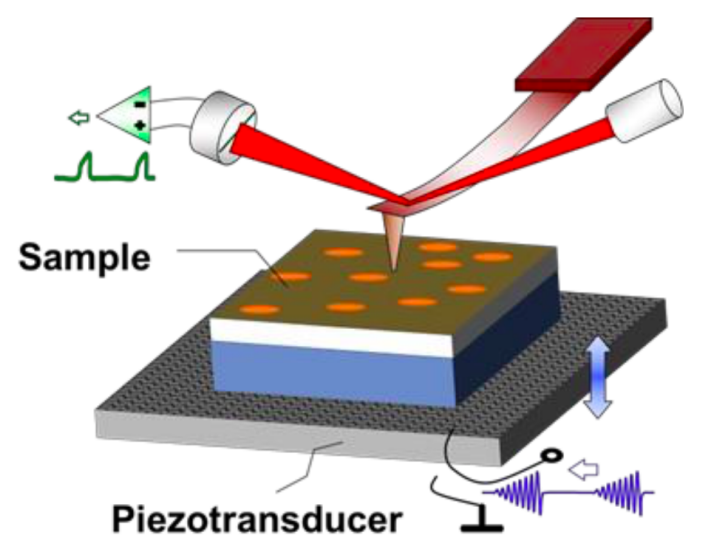

Figure 2.3: Schematic of the UFM operation principles. The sample is oscillating vertically at ultrasonic frequencies by a piezotransducer and the response is measured in the laser deflection signal.

\subsubsection{History and principles of SThM}

To overcome the STM limitation requiring an electrical conductive surface, several techniques were developed using different physical mechanisms to map a surface topography. The first SPM using heat as interacting agent between the probe and the sample was developed by Williams and Wickramasinghe[3]. The method used a heated thermocouple sensor as a nearfield noncontact probe. When the tip-sample distance is changing, the heat flux changes as well and the distance is rectified to maintain the same heat flux. Thanks to the low air thermal conductivity compared to solid materials, this method was possible and almost independent on the sample thermal conductivity. We highlight that the technique didn't require a laser for the feedback. Later, using a similar system, the same group reported imaging thermal conductivity contrast using temperature dependence of the contact potential of Kelvin Probe Microscopy 40 . Combining AFM and a temperature sensor, today's SThM was born.

An SThM consists generally of the following parts 41] (see Fig. 2.4):

- a sharp probe acting as a temperature sensor; 
- a heat source either embedded in the probe itself, external from electromagnetic radiation or part of the sample itself (e.g. a microscale heater);

- a probe-sample force transducer such as a cantilever;

- Feedback systems for the force and/or the thermal probe.

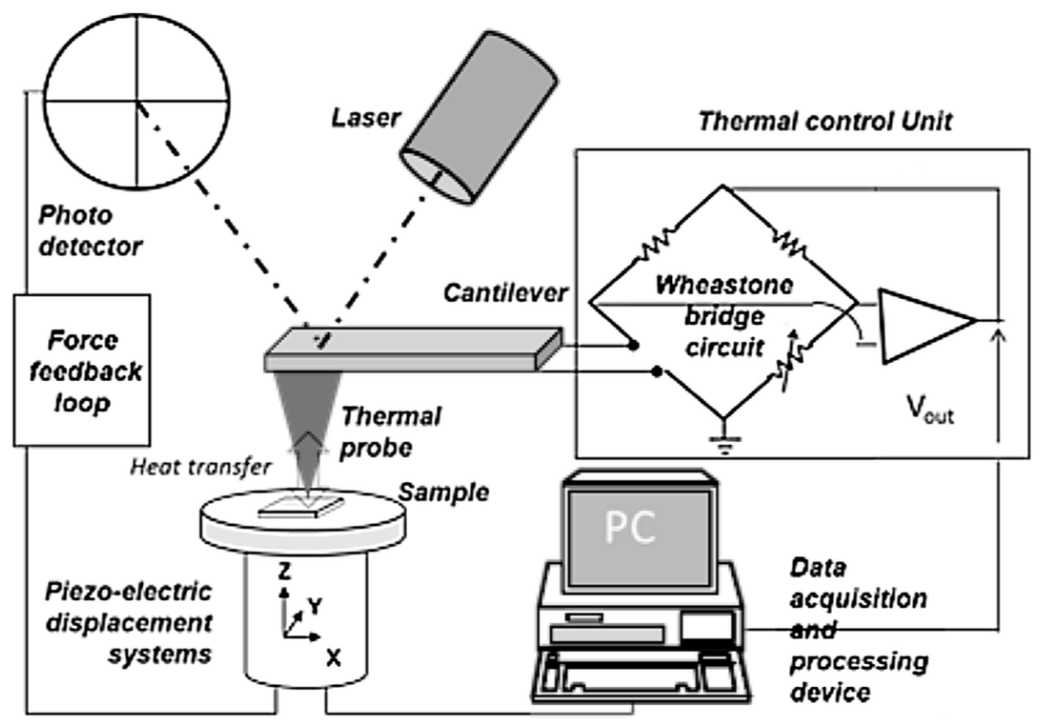

Figure 2.4: Standard SThM components using the AFM force feedback loop for positioning and a thermal control unit, usually composed of a Wheatstone bridge, for measuring the thermal probe electrical resistance 8$]$.

Later, various thermal probes were developed, giving birth to different trends of SThM practice depending on the temperature dependence phenomena used for probing heat transfer. Four probes categories can be outlined: thermocouple, resistive,thermally expansive and fluorescence based 8,42 . We quickly present the first three catergories. Also, we note that recently a cryogenic sensor based on a superconducting quantum interference device (SQUID) was introduced 43.

\section{Thermovoltage probes}

Thermocouple probes are based on thermoelectric principles for the determination of the sensor temperature. Different methods are possible. For example, Sadat et al. 44 used the creation of a junction between a Pt tip and a sample to map 
the temperature field of a metallic surface. However this requires conductive surfaces.Therefore, a thermocouple junction is usually created at the tip apex. For example, a gold-palladium [45] junction or gold-chromium $[46]$ were reported. The main advantage of these probes is the proximity of the sensor with the sample. This proximity enabled measurements of enhanced radiative transport in the extreme near field 23$]$.

\section{Resistive probes}

Quickly after the first SThM developments, resistive probes appeared 47$]$. The Wollaston wire probe 41] has been attractive for many years thanks notably to its electrical and mechanical robustness. However, with the rise of SThM applications in nanosciences, it could not be used for lateral resolution below $\sim 1 \mu \mathrm{m}$ due to its large active area.

All resistive probes operate on a similar principle of temperature dependence of the electrical resistance of a sensor:

$$
R_{p}(T)=R_{p 0}\left(1+\alpha\left(T-T_{0}\right)\right)
$$

where $R_{p 0}$ is the electrical resistance of the probe at a reference temperature $T_{0}$ and $\alpha$ is the temperature coefficient of the electrical resistance. Therefore, the resistive element can serve as just a sensor in passive mode for thermometry or as a heater/sensor in active mode for thermophysical properties measurements. We develop details of the passive and actives modes in the following section.

In this work, all experiments were performed using resistive probes. Therefore, we develop the two types of probes which were used, the palladium probe or KNT probe and the doped silicon probe.

Palladium probes Palladium probes consists of a silicon nitride cantilever (formerly silicon oxide) [48] with gold pads and contacts leading to two palladium films joining at the tip end (see Fig. 2.5a). Thanks to its tall triangular shape 
tip $(10 \mu m)$, the cantilever is partially decoupled from the sample. Also, thanks to its design, most of the heat is generated at the tip, it allows operation in ambient conditions. This is an advantage compared to the doped silicon as we will see. Finally, its $50 \mathrm{~nm}$ radius of curvature and relatively fast time response (few tens of $\mu$ s) have made this probe attractive and widely used. However, as we will develop in chapter 3, a comprehensive model is required to fully account for the dimension of the tip.

Limitations arise from its electrical capabilities. Electron migration limits the maximum current that the palladium film can sustain to around $1.5 \mathrm{~mA}$ [48]. Higher currents lead to resistance drift and ultimately burnt films as seen in figure 2.5b. Also, the cantilever being made of gold films on silicon nitride create an effective bimorph because of the different thermal expansion coefficient of the two materials. Variations of the surrounding temperatures change the laser deflection when used in AFM configuration. New probe designs were developed to counter this effect using a new geometry of the cantilever 49 .
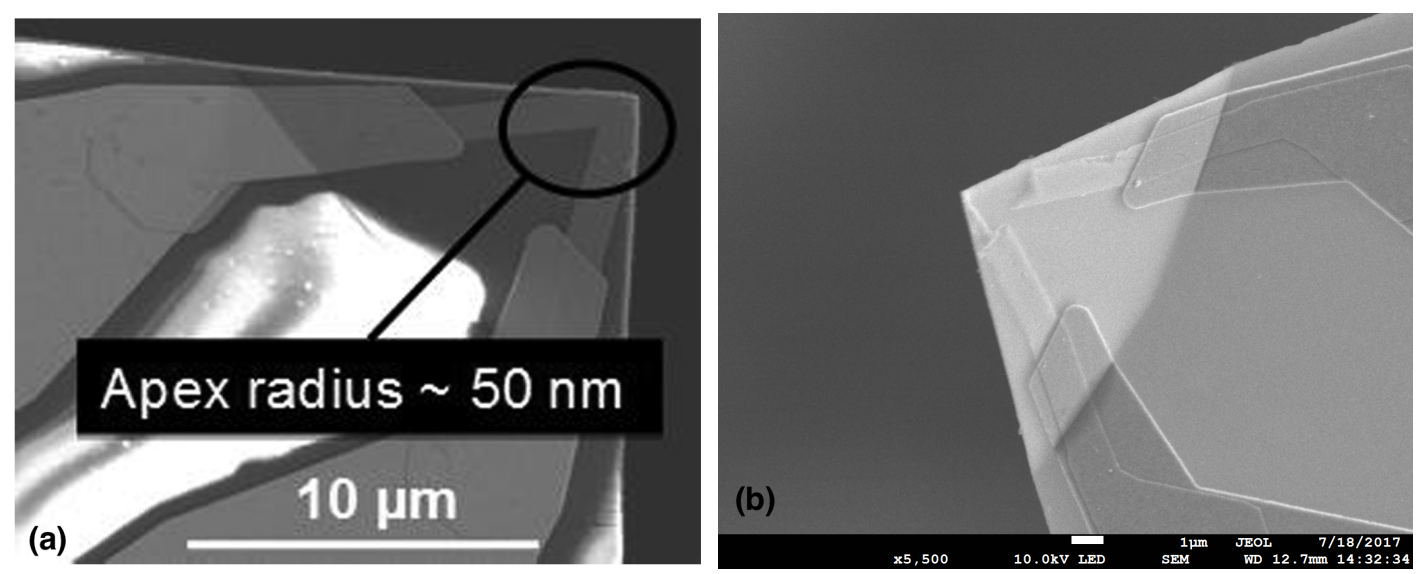

Figure 2.5: (a) SEM image of the KNT probe 8 with the Pd film joining at the tip end with an apex radius of $50 \mathrm{~nm}$ and (b) Burnt KNT probe after excessive current was applied to the Pd film.

Doped silicon probes Initially designed by IBM for nanolithography purposes such as high density data storage, the cantilever is made of two highly doped $\mathrm{Si}$ legs connected at their end to a low doped resistive region (see figure 2.6). Using a similar fabrication process to regular AFM probes, the tip placed below the 
resistive element has a conical shape (around $5 \mu \mathrm{m}$ high). Its apex radius can reach $10 \mathrm{~nm}$. Unlike for the palladium probe, the maximum currents that the doped silicon probe can sustain are much higher and this leads to high excess temperature, more than $150 \mathrm{~K}$ according to the manufacturer. However, at high current, the resistance change is not linear any more and second order corrections need to be taken into account.

Furthermore, the sample is separated from the heating/sensing region by the conical tip which is highly thermally resistive due to its sharpness. Therefore, when used in ambient conditions, the effective volume probed is made of a much bigger volume than the one just below the apex[50]. Vacuum environment is essential for using these probes in SThM at high resolution.

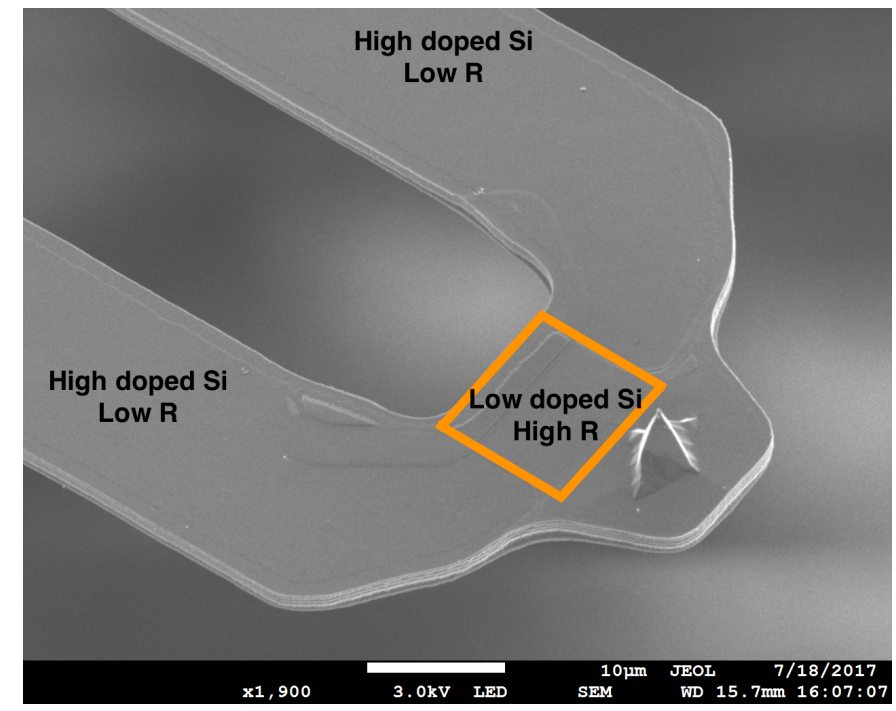

Figure 2.6: SEM of the doped silicon probe with high doped, low electrical resistance, Si regions and a low doped, high electrical resistance, Si implant used as a heater.

\section{Thermal expansion}

A last category of SThM uses thermal expansion for monitoring heating processes. Invented slightly after the first SThM developments by Majumdar et al.[51, 52], it monitored the deflection of an standard AFM probe when an embedded resistor was heated by Joule heating. Later, thermal expansion was implemented using an infrared beam where generally the laser source is pulsed and therefore generates 
a heating oscillation within the sample material. Creating a thermal expansion movement, this oscillation can be detected by the AFM probe deflection 53 . We note that this technique also enables spectroscopic measurements of a sample 54 for example for chemical and biological investigations.

Thermal expansion SThM can also rely just on the probe itself when two metals are used for the cantilever. The different thermal expansion coefficients can create significant bending of the cantilever which in turn can be detected by regular AFM detection $55 \mid$. Because the cantilever bending acts as a sort of mechanical amplifier, very small temperature changes can be detected, as small as $10^{-5} \mathrm{~K}[56$. King and Pop et al. used thermal expansion SThM to detect thermoelectric effects in nanodevices such as graphene[57], carbon nanotube[58], and memory devices[59].

\subsubsection{Basic operation possibilities}

As all the following work has been performed using resistive probes, we explain here the methodology commonly used in SThM setups. Traditionally, the resistive probe is part of a resistor bridge, e.g. a Wheatstone bridge (see figure 2.7). This configuration allows the detection of small resistance variation. Different bridge balance conditions are possible through a parallel variable resistor $\left(R_{e}\right.$ on the figure). The bridge can be balanced when power is applied to the probe or the bridge is balanced at low power. Both DC and AC operations are possible. However, AC setups are believed to be more sensitive, have lower drift and smaller uncertainties 60 . AC measurements can be performed with the $3-\omega \operatorname{method} 61,62$ where the third harmonic of the bridge output can be shown to directly relate to the probe self-heating and therefore the heat losses 63. 


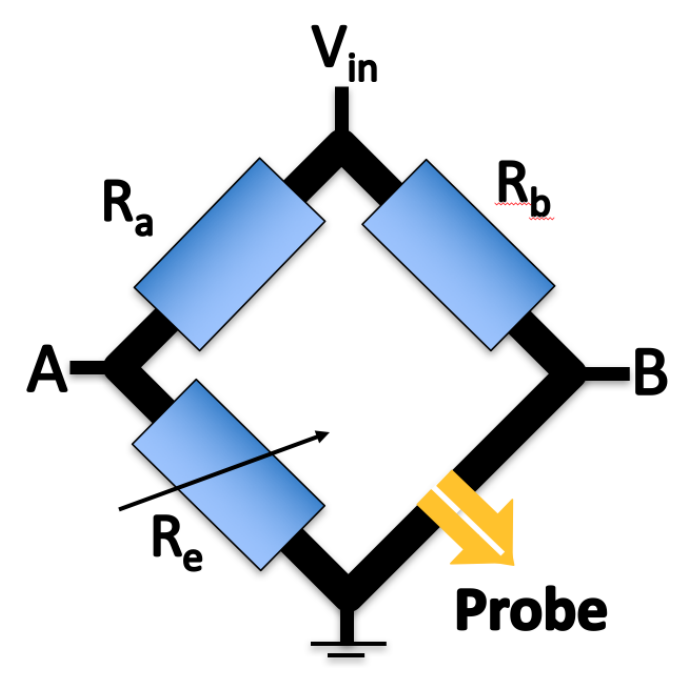

Figure 2.7: Typical Wheatstone bridge for a SThM probe. $R_{a}$ and $R_{b}$ are fixed resistors and $R_{e}$ is variable to balance the probe resistance. The bridge is biased by $V_{i n}$ and the bridge output is the difference between $A$ and $B$.

Three typical applications can be highlighted: thermometry, thermal conductivity/conductance characterization and for local heating purposes 8 . In all these applications and for most SThM setups, the probe-sample system is similar (see figure 2.8). In a common situation the microscope temperature $T_{m}$ and the sample temperature $T_{\text {sample }}$ are equal. Therefore, when some heat $Q$ is provided at the sensor, two heat channels are possible: through the cantilever thermal resistance $R_{p}$ and through the tip to the sample via the tip-sample resistance $R_{t s}$. We develop further the different physical mechanisms for heat transfer between the tip and the sample (see section 2.3.3). 


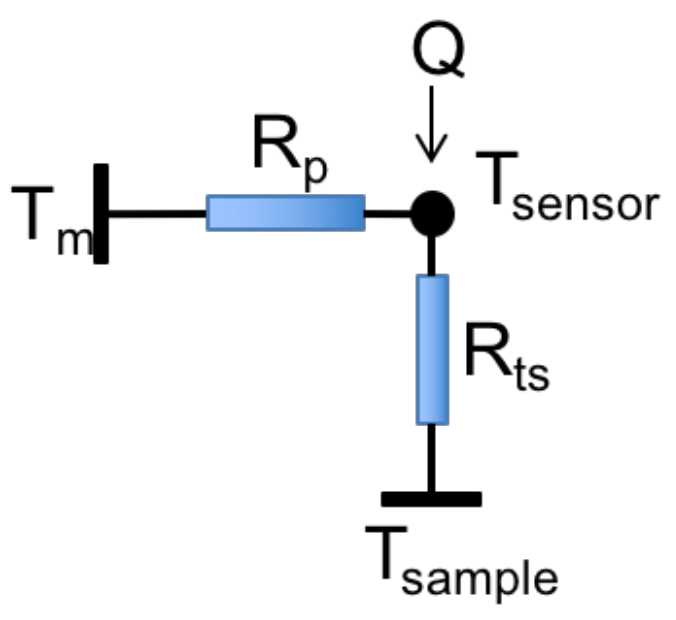

Figure 2.8: Schematic of the probe-sample system. Some heat $Q$ is supplied at the sensor which is at $T_{\text {sensor }}$. Sample and microscope are at $T_{\text {sample }}$ and $T_{m}$, respectively. Heat can flow through the cantilever thermal resistancce $R_{p}$ and through the tip-sample thermal resistance $R_{t s}$.

\section{Thermometry}

For thermometry application, $R_{t s}$ is a significant unknown as it can be difficult to estimate and measure. It also suffers from artefacts such as topography-related contrast 64 which arise due to modulation of the effective probe-sample contact area. In most cases, $R_{t s}$ needs to be calibrated and a calibration factor is applied to extract the sample temperature $T_{\text {sample }}$ such as in Kim et al. [65] even when the sensor is in direct contact with the sample.

To overcome this limitation, different methods were developed: the null-point method $[66]$ and the dual-sensing technique of Menges et al.|67]. The null-point method, which has the advantage of being applicable in air, is based on a double scan approach [68, 69]. The first scan is performed out of contact immediately before the contact and the second scan is in contact with the sample (see figure 2.9 . 

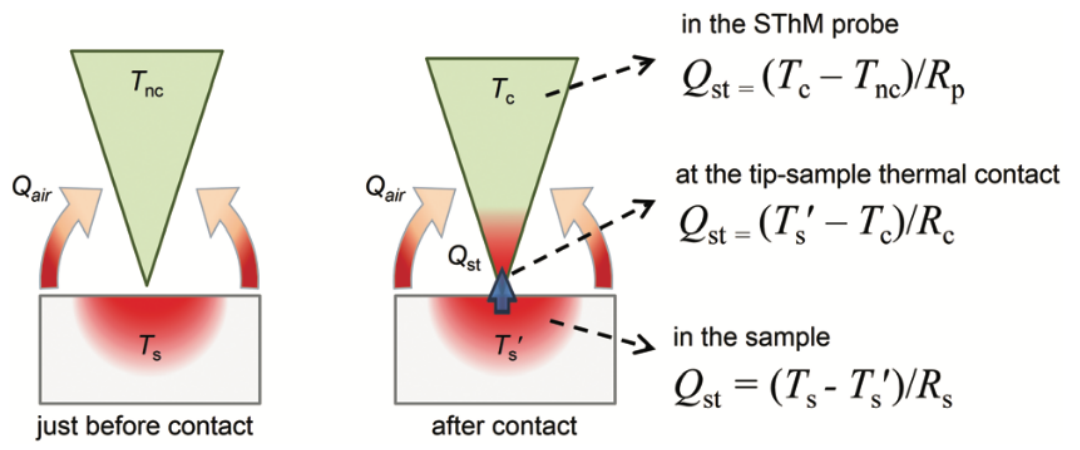

Figure 2.9: Null-point method principles 68. Two measurements are performed one just before contact and one in contact. This allows to eliminate the parasitic air heat transfer, $Q_{a i r}$, contribution and measure sample-tip heat transfer, $Q_{t s}$.

Similar to the null-point method, Menges et al. [70] have mapped the temperature of a silicon nanowire by a double scan technique with a first scan at room temperature and a second one with the nanowire heating the sample. Modulating the temperature of the sample enables the determination of both the thermal resistance and the temperature of the sample. They further improved their technique by applying an $\mathrm{AC}$ bias creating an oscillating temperature distribution and using lock-in detection at the bias frequency, they achieved $7 \mathrm{mK}$ resolution 67,71 .

\section{Thermal conductance measurements}

When the sensor is heated, the heat flux to the sample can be measured. However, the sample temperature is often assumed to be equal to the heat sink temperature. It was shown that this assumption holds for high thermally conductive materials 50 but, for lower ones, it might not be the case, especially when measuring in ambient conditions. For example, polymers get heated by the probe before it enters into contact.

SThM can be a powerful tool to investigate heat transfer in nanostructures. Thin films on substrate $[72,74]$ or even $2 \mathrm{D}$ materials $68,75,76]$ have been investigated. Complex structures can also be scanned as long as the surface is smooth enough to avoid dominance of topography-related artefacts 77.79]. 


\section{Local heating element}

A third application uses SThM in active mode to create a local temperature variation. Most uses reported are for phase change temperature such as glass transition temperature or melting temperature of polymers 41, 80]. Lee et al.|81 used a thermocouple probe to induce local heating in a Si p-n junction and map with nanoscale resolution the Seebeck coefficient. We will show in chapter 7 similar measurements using the SThM as a local heating element allowing to measure thermoelectric effect in graphene constrictions.

\section{Environments}

These modes of operations have been reported in various environmental conditions. Aside from ambient air conditions, pressure dependence has been investigated 82 as well as the impact of humidity 83 and pressure 84 . Vacuum and high vacuum SThM have also been developed. Vacuum operations are usually more quantifiable as both liquid meniscus and air conduction are removed therefore increasing the thermal and spatial resolutions. Kim et al. used an ultra-high vacuum technique and obtained $15 \mathrm{mK}$ temperature resolution and $10 \mathrm{~nm}$ spatial resolution.

Under liquid immersion, SThM has also been demonstrated. A liquid environment provides very gentle scanning and is therefore suitable for soft materials such as biological samples 85 . Forces between the probe and the sample are about 10 times smaller than in air 86$]$. Aigouy et al. [87] developed a setup working in liquid (70\% water and $30 \%$ glycerol) using a fluorescence probe to monitor self-heating of a nickel microheater. This technique thus requires an active sample which limits its uses. More recently, Tovee et al. $[88]$ designed an immersion SThM (iSThM) working with a resistive probe. The design is shown on figure 2.10. In order to avoid corrosion of the probe, they used dodecane as surrounding liquid. In their experiments, they achieved $30 \mathrm{~nm}$ spatial resolution. 


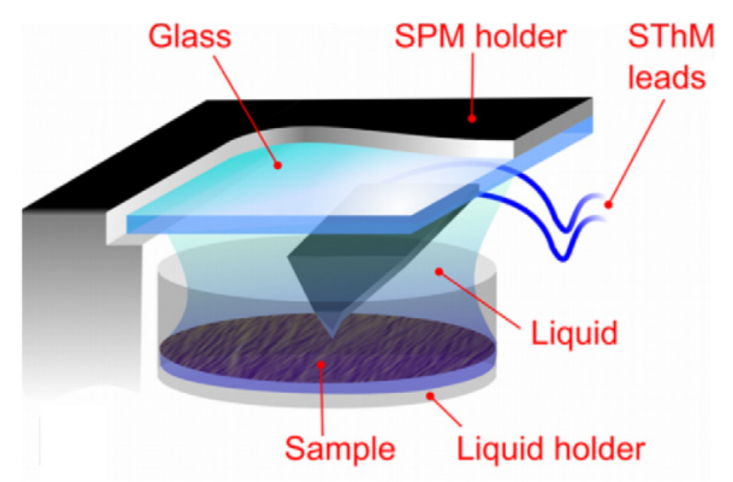

Figure 2.10: Immersion SThM setup design|88|. The liquid forms a meniscus between the liuquid holder and a glass slide above the SThM tip.

\subsubsection{Heat transfer mechanisms in the tip-sample system}

Each system has a different configuration be it a different probe, a different operation mode or environment. However, the physics underlying these systems is, in the majority of cases, similar. We review in this section the different heat transfer mechanisms between the tip and the sample. Four mechanisms are generally present: heat conduction at the solid-solid contact, transfer through the water meniscus, air or surrounding medium heat transfer and thermal radiation. Depending on the environment, some heat transfers can be removed. For example, in vacuum, air is removed and therefore the water meniscus is greatly reduced. In liquid or in a specific atmosphere, water meniscus is removed as well. The SThM output is ultimately depending on these mechanisms and a correct interpretation of the data depends on the understanding of the phenomena at stake. 

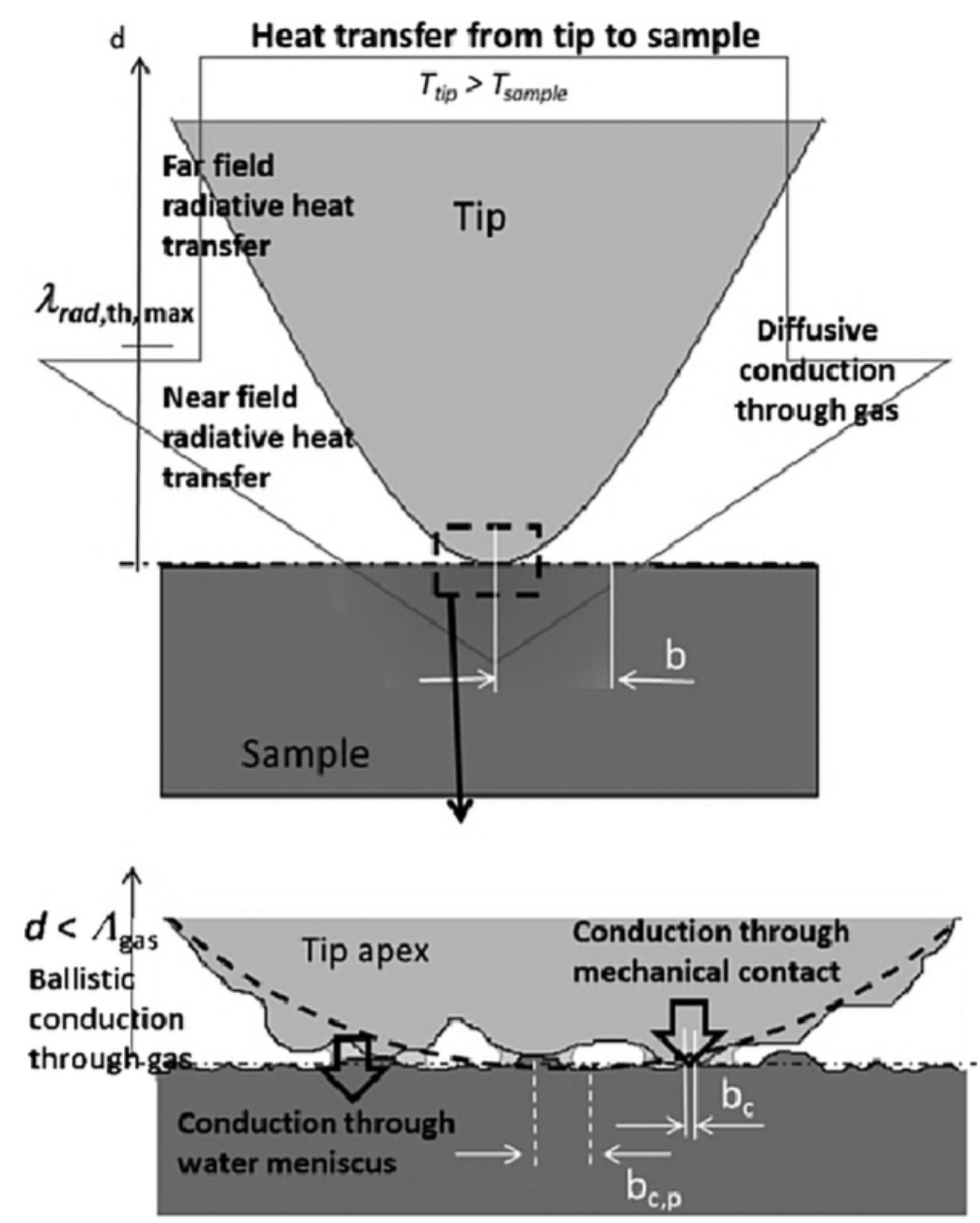

Figure 2.11: Schematics of the heat transfer mechanisms in the tip-sample system at different lenght scales $[8]$. The main components are diffusive and ballistic conduction through the surrounding gas, conduction through mechanical contact and through the water meniscus.

\section{Solid-solid contact heat conduction}

When the mechanical contact is created, heat can be transferred through the solidsolid interface. We already discussed the nanoscale size effect on interface thermal transport earlier. A major challenge for any SThM measurement is to determine the nature of the contact. It is also important to ensure a constant solid-solid junction otherwise signal variations would be difficult to attribute to the contact change or to the probed material itself. Aside from carrier propagation through the interface, crucial experimental factors will affect the solid-solid heat conduction: contact area and sample roughness.

The physical contact area is very difficult to measure and the true nature of the 
contact probably is better represented as a multiasperity contact 8 . Mechanical contact theories can be applied to gain some insights and find approximations of the contact radius. Also, SEM imaging can be a complementing technique to measure the tip apex radius. With the contact radius, the interface thermal resistance can be estimated by normalising the interface resistance per unit area:

$$
R_{\text {int }}=r_{\text {int }} / \pi a^{2}
$$

where $a$ is the contact radius and $r_{i n t}$ is the interface thermal resistance or boundary thermal resistance and has units $\mathrm{m}^{2} \mathrm{KW}^{-1}[8,89]$. This approach is, however, slightly artificial as there is no proof that the macroscopic interface thermal resistance simply scales with the contact area.

Roughness of both tip and sample can strongly influence the thermal transport. In some sense, it corresponds to a contact area issue. Gotsmann and Lantz 90 studied the forces dependence of the tip-sample thermal transport for various tip roughnesses in vacuum. They argue that the thermal transport is quantized across atomic-scale contacts and developed a model taking into account the nanoscale roughness effect. Pettes and Shi 91 studied similar systems for a Si-Si contact and modelled the interface thermal resistance using a nanoconstriction model.

\section{Water meniscus}

As two objects form a contact in ambient conditions, a water meniscus appears due to the presence of water in ambient air and capillary condensation. The size of this meniscus depends on several parameters: materials, humidity and temperature. As this will increase the effective thermal contact between the probe and the sample, it reduces the spatial resolution. Little literature exists on the subject. Shi and Majumdar 92 reported that, for a metal surface, the water meniscus conduction was of the same order of magnitude as the solid-solid conduction. Assy and Gomès 83 studied heat transfer through this meniscus for the doped silicon and the palladium probes. The found that the meniscus conductance was dependent on the 
probe temperature and could reach $6 \%$ of the total conductance for the palladium probe and $4 \%$ for the doped silicon probe.

\section{Gas and surrounding medium heat transfer}

When operating in ambient conditions, air heat transfer is a major component of the tip-sample system and depends on the probe design. For example, the doped silicon probe suffers from poor sensitivity when operating in air[50] because of the distance between the heater/sensor to the sample. Therefore, the palladium probes are more suitable. For a Wollaston wire probe, up to $65 \%$ of the heat transferred to the sample is carried by the surrounding air $[8$. Three mechanisms can occur depending on the heat-sample distance. When considering large distances, air convection transports heat by gas flow. At distances smaller than a few micrometers, heat diffusion becomes the dominant mechanism. Finally, when considering a few hundreds of nanometers, ballistic conduction occurs as the carrier mean free path of air is around $70 \mathrm{~nm}[8]$. For a palladium probe, air heat transfer is in the conduction regime partly diffusive and partly ballistic. It is therefore difficult to model and measure such effects.

\section{Thermal radiation}

As the length scales considered are similar and smaller than the typical wavelength $\lambda$ defined by Wien's displacement law $(\lambda T=2897 \mu \mathrm{m} \mathrm{K})$, classical and near-field radiation transport must be considered. In fact, it has been demonstrated that near-field transfer can be increased by orders of magnitude compared to far-field 23 , 93. However, radiative heat transfer requires particular conditions to acquire intensity such as similar optical spectra of the materials $[94$. Also, when working at room temperature, radiation is considered negligible 42,84 . The importance of radiation in SThM has been and remains under discussion. 


\subsubsection{What is measured?}

Before moving to the last section of this literature review and after this short review of SThM techniques, it is worth thinking about the physical nature of the tip-sample system. When the hot SThM tip enters in contact with a sample, heat propagates to the sample. The amount of heat transferred depends on parameters such as the contact area or the material properties and temperature. An interesting comparison can be made between an SThM probe and our body's temperature perception ability. If we place two blocks of different materials, let's say one of aluminium and one of glass, and ask someone which block is colder, the answer would be the aluminium one. Although, the two blocks are at the same temperature. The skin senses heat fluxes. When contacting the aluminium, because of its larger thermal conductivity than the glass, a greater heat flux occurs than when the finger touches the glass.

This simple example shows that temperature and heat flux measurements have always been deceiving. An SThM experiment output comparing heat conductance of gold and silicon oxide can be surprising and counter-intuitive because of the difficulty to interpret the result itself. As we saw many parameters influence this output and depending on the measurement procedure, different results are possible even in very simple cases. Temperature itself can be hard to define because of some non-equilibrium processes at stake. Also, the temperature distribution across the tip-sample interface is discontinuous because the temperature cannot be defined at the interface. Furthermore, at the nanoscale, when does a thin film become an interface? For example, can we define the temperature of a graphene layer on silicon oxide? The question is whether thermodynamic equilibrium can be assumed. As we will see in the next section, to face these questions, we are often left with classical classical concepts that probably need revision in the future.

Operating in active mode, some reports demonstrated the ability to measure thermal conductivity $8,95,96$. However, the method always include a calibration step against standard samples. There is, however, a bias in such a method. The 
calibration set might just include one type of material, for example only phononic materials. Also, the sample probed might have a different surface state (for example different roughness) than the calibration set and the measurement would then lead to a systematic error. This difficulty to measure thermal conductivity with SThM has two origins:

- Thermal conductivity as a local property is fully defined for bulk materials.

- SThM measures heat fluxes. Depending on the formalism and setup used, the output of an SThM experiment is thermal resistance or conductance between the heater/sensor and the sample heat sink. Sample heat sink temperature and heater temperature are related by the thermal resistance $T_{\text {heater }}=T_{\text {sample }}+R_{t s} Q_{t s}$. The thermal resistance $R(t s)$ is the effective thermal resistance between the sensor and the heat sink and thus comprises several components including the thermal resistance of the sample itself which in many cases is the goal of the SThM measurement.

\subsection{Modelling and simulations}

Since SThM measures thermal resistances, it is of interest to attempt some modelling of such resistances. Even if thermal resistance can provide crucial information about nanoscale heat transfer, it can also be used to extract other quantities such as thermal conductivity or interface resistance. The process is therefore in two steps. First, the thermal resistance is measured and only then it can be modelled to extract other quantities. The quantities extracted represent often effective quantities as they are obtained through a model which might not represent accurately the physics of the system. We address two modelling techniques in this section as they are the ones used in the rest of this thesis: finite elements modelling (FE) and analytical formulations. 


\subsubsection{Finite Element Analysis compared to analytical formu- lations}

As computing power increased drastically, it is now possible to create complex FE models including sophisticated geometries assigning various materials to different regions. For example, the full probe-sample system can be modelled, for example, to study radiation thermal transport|93], immersion SThM and probe sensitivity in various environments [50, 88], time-constant of the probe and tip-sample resistance 65] or even topography-related arterfacts 97. Thus FE modelling is becoming a necessary tool for investigating tip-sample systems complementary to analytical analysis as it handles difficult geometries and multiphysical problems. In many cases, these models are still based on macroscopic laws and thus do not represent the exact nature of the system. For example, air heat transfer modelling is challenging when dealing with micrometer length scales. Effective parameters can be introduced to account for some nanoscale effects.

However, the specificity and complexity of these models and FE tools make them still difficult to use on a daily basis. As the goal of the SThM measurements modelling is to extract quantitatively physical properties, an alternative is to model only heat propagation within the sample. This reduces the computing time and removes some difficult aspects of FE for nanoscale systems such as effective parameters for the air heat transfer. For example, El Sachat et al.98 used FE models to compare with experimental data and extract thermal conductivities of $\mathrm{Si}_{1-x} \mathrm{Ge}_{x}$ nanowires. Martinek et al. developed FE models to fit onto SThM of supported and suspended thin films 99 .

Modelling only the sample can be an attractive approach as various geometries are possible even complex multilayer packaging for light-emitting diode 100 . Nevertheless, FE is relatively time consuming, especially if parameters analysis is required. Large meshes are also required even for simple film-on-substrate geometry. An alternative is provided by analytical formulations which, as we will see, can also be complex but, for simple cases, are more efficient and straightforward. 


\subsubsection{Spreading resistance of layered systems}

Heat spreading analysis is crucial for the packaging industry 101 and started in the 1950s for applications in electronics. However, it requires advanced mathematical treatment. We will explain the solution for the semi-infinite case of a single layer on a substrate as derived by Dryden[102]. Later, Yovanovich 103 and Muzychka 104 developed this solution for various systems.

The model uses cylindrical symmetry as in figure 2.12. The heat spot has a radius $a$ and is in perfect thermal contact with an infinite half-plane with a material 1 of thickness $t$ and thermal conductivity $k_{1}$. This layer rests on a substrate of material 2 with a thermal conductivity of $k_{2}$. The equation to solve is the Laplace equation for the temperature $T$ in both regions.

$$
\begin{array}{ll}
\nabla^{2} T_{1}=0 & r>0,0<x<t \\
\nabla^{2} T_{2}=0 & r>0, x>t
\end{array}
$$

In an axisymmetric coordinates system, the Laplacian is

$$
\nabla^{2}=\frac{\partial^{2}}{\partial r^{2}}+\frac{1}{r} \frac{\partial}{\partial r}+\frac{\partial^{2}}{\partial x^{2}}
$$

Then, requiring continuity of the temperature and heat flux at the interface between the two materials

$$
\begin{array}{rr}
T_{1}=T_{2} & r>0, x=t \\
k_{1} \frac{\partial T_{1}}{\partial x}=k_{2} \frac{\partial T_{2}}{\partial x} & r>0, x=t
\end{array}
$$

Boundary conditions are also required at the surface:

$$
\begin{array}{cl}
\frac{\partial T_{1}}{\partial x}=-f(r) & a>r>0, x=0 \\
\frac{\partial T_{1}}{\partial x}=0 & r>a, x=0
\end{array}
$$


with the isothermal flux distribution $f(r)$ :

$$
f(r)=\frac{Q}{2 \pi a\left(a^{2}-r^{2}\right)^{1 / 2}}
$$

and assuming that the temperature field vanishes as we go away from the heat source, that is:

$$
\left(r^{2}+x^{2}\right)^{1 / 2} \rightarrow \infty \Rightarrow T_{1} \rightarrow 0 \quad \text { and } \quad T_{2} \rightarrow 0
$$

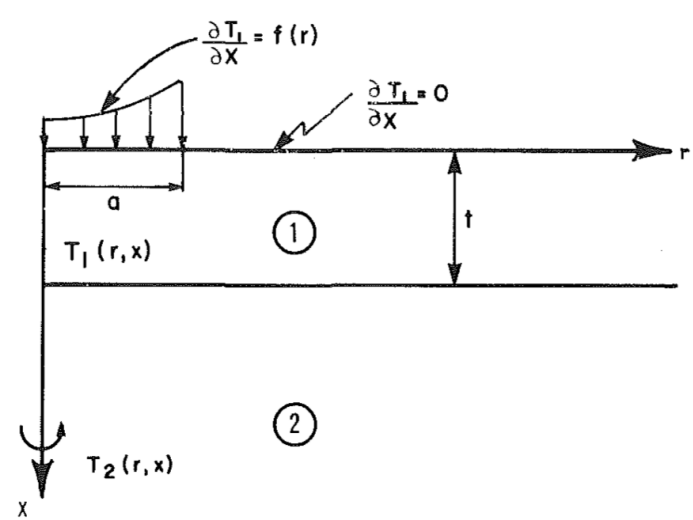

Figure 2.12: Model of a heating spot of radius $a$ on a half-plane coating of thickness $t$ on a substrate with boundary conditions 102. Note that the system has cylindrical symmetry.

Then using a Hankel transform (details can be found in the references) on the Laplace equations and continuity and boundary conditions, the temperature field in the first material is described by

$$
T_{1}(r, x)=\int_{0}^{\infty} \xi \bar{T}_{1}(\xi, x) J_{0}(\xi r) d \xi
$$

where $\bar{T}_{1}$ is the expression for $T$ in the Hankel space and $J_{0}$ is the Oth order Bessel function of the first kind.

The spreading resistance, or constriction resistance $R$, of a hotspot is defined as

$$
R=\frac{\Delta T}{Q}=\frac{\hat{T}}{Q}
$$


where $\Delta T$ is the temperature increase and $Q$ is the heat flux through the hotspot. The temperature increase is equal to the average temperature $\hat{T}$ of the contact spot:

$$
\hat{T}=\frac{1}{\pi a^{2}} \int_{r=0}^{a} T_{1}(r, 0) 2 \pi r d r
$$

We then arrive at the solution for the thermal spreading resistance of a layer on substrate.

$$
R_{s p r}=\frac{1}{\pi k_{1} a} \int_{0}^{\infty}\left[\frac{1+K \exp (-2 \xi t / a)}{1-K \exp (-2 \xi t / a)}\right] J_{1}(\xi) \sin (\xi) \frac{d \xi}{\xi^{2}}
$$

where $J_{1}$ is the 1 st order Bessel function of the first kind and $K$ is defined as

$$
K=\frac{1-k_{2} / k_{1}}{1+k_{2} / k_{1}}
$$

This solution is valid however only for isotropic thermal conductivities and a perfect thermal interface. More complexity can be added when considering interface resistance at the layer-substrate boundary and orthotropic systems. Orthotropic materials are a class of anisotropic where a property is different depending on the direction of measurement. However, orthotropic materials still conserve some symmetries. For example, graphite is an orthotropic material with different in-plane and out-of-plane thermal conductivities. To extend the solution developed above, Muzychka used the method of stretched coordinates 105 107. This elegant method transforms the orthotropic system into an isotropic one thus making it possible to use the same solution already derived.

The transform is sketched in figure 2.13. In the first layer the $z$ direction becomes $\xi=z / \sqrt{k_{1, z} / k_{1, x y}}$ and in the second layer it becomes $\zeta=z / \sqrt{k_{2, z} / k_{2, x y}}$. Then orthotropic conductivities and thicknesses are used to define isotropic ones:

$$
\begin{array}{ll}
k_{1}=\sqrt{k_{1, x y} k_{1, z}} & \bar{t}=t_{1} / \sqrt{k_{1, z} / k_{1, x y}} \\
k_{2}=\sqrt{k_{2, x y} k_{2, z}} & \bar{t}=t_{2} / \sqrt{k_{1, z} / k_{1, x y}}
\end{array}
$$


In the case a of semi-infinite single layer on a substrate, we can include the interface conductance $h_{c}$ in the isotropic thickness and we are left with:

$$
k_{1}=\sqrt{k_{1, x y} k_{1, z}} \quad \bar{t}=t_{1} / \sqrt{k_{1, z} / k_{1, x y}+k_{1} / h_{c}}
$$
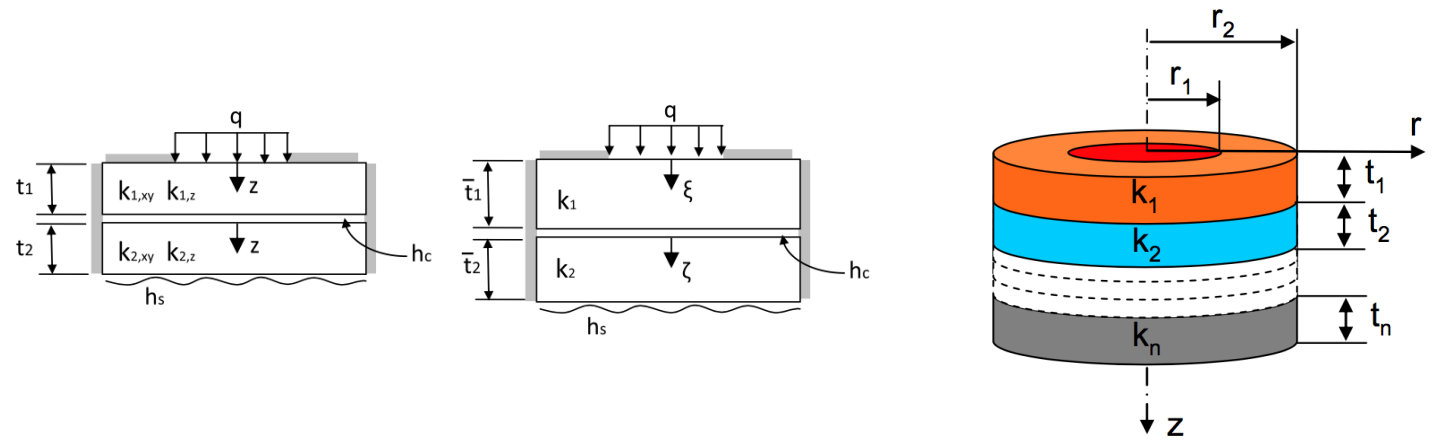

Figure 2.13: Transformation from an orthotropic system to effective isotropic properties 107] and multilayers fluxtube model[108].

Such models were originally derived for compound flux tubes including therefore lateral dimensions with adiabatic boundary conditions on the side of the tube. These solutions are usually not useful for nanoscale SThM measurements as the size of the contact spot is much smaller than the lateral dimensions of the films studied. Hence, the derivation is not developed here. However, they can be of interest as some models were derived for multilayer systems. Palisoc et al. 109 demonstrated a solution for a four layers system, also with embedded heat source 110. However, their solution is not straight forward to implement and requires large computing time rendering it unsuitable for parametric studies 100. Similarly to Muzychka 104, $\mathrm{Ha}[108$ s solved the Laplace equation for a multilayer structure and computed parameters up to four layers tube (see figure 2.13). We simply state the solution here:

$$
R_{\text {multilayer }}=\frac{1}{\pi r_{2}^{2}}\left(\sum_{i=1}^{n} \frac{t_{i}}{k_{i}}\right)+\frac{2}{k_{1} \pi r_{1}} \sum_{l=1}^{\infty} \frac{J_{1}\left(\frac{r_{1}}{r_{2}} \alpha_{l}\right)}{\alpha_{l}^{2} J_{0}^{2}\left(\alpha_{l}\right)} \phi_{n}
$$


with

$$
\alpha_{l}=\frac{\beta_{l}}{4}\left(1-\frac{6}{\beta_{l}^{2}}+\frac{6}{\beta_{l}^{4}}-\frac{4716}{5 \beta_{l}^{4}}+\frac{3902418}{70 \beta_{l}^{4}}\right)
$$

and $\beta_{l}=\pi(4 l+1)$.

We note that Muzychka also derived equations for the thermal resistance of non-circular heat sources and show small dependence on the shape of the hotspot 104. Also, they addressed the question of a moving heat source on an half space which could be interesting for SThM measurements 111. Gholami et al.[112] studied similar systems including several hotspots arbitrarily located.

The spreading resistance models exposed above were directly used by SThM experimentalists to obtain thermal conductivities of layer on substrate systems. The first report we found was Gomes et al.|73| using an analytical solution to study meso-porous silicon. Then, all reports to dates used Dryden's solution or Yovanovich's and Muzychka's adaptations. For example, Menges et al. [113] investigated orthotropic transport in graphene and few layers graphene on different substrates. This work was also used by other groups studying thermal transport in $2 \mathrm{D}$ materials. In very similar systems, Tortello et al. $[76]$ studied reduced graphene oxide flakes and Hwang et al.|68], suspended graphene disks and supported graphene on silicon oxide. Sadeghi et al.|114 used the same model to analyse heat spreading of graphene devices on flexible substrate. Finally, Juszczyk et al. $|74|$ made a more general use of these solutions for oxide layers. We also note that these analytical solutions can be useful for sample design as for example Ge et al. who developed a topography-free sample for SThM probe characterization. The use of these analytical diffusive models for nanometric samples can be questioned. In many systems diffusive transport might not be relevant or at least combined with a ballistic contribution. However they provide a useful framework of understanding. Using effective quantities is useful to compare different experiments performed in similar conditions. Comparison with other methods can be more challenging but in some case outputs similar results. 


\subsection{Summary}

Heat transfer presents great challenges from an experimental and theoretical point of view. Various techniques were developed trying to gain insights into nanoscale systems. We reviewed the main differences between macroscale and nanoscale heat transfer. Nanoscale SPM techniques and SThM demonstrated their strength to address commonly faced issues of standard techniques. Various types of SThM and the different environments were explained as well as the various components of a SThM measurement. However, SThM still demands experimental developments and theoretical understanding in order to achieve quantitative and reproducible measurements. Once a good understanding of the measurement process is established, various modelling approaches are available. We limited the approach to spreading resistance analysis of simple geometry and finite elements modelling. As we demonstrate in the following chapters, a combination of SThM setup developments, measurements procedures and modelling results provide a robust approach for quantitative nanothermal measurements of nanostructures in SThM. 


\title{
3. SThM experimental models and
}

\author{
setups for exploring nanoscale heat
}

\author{
transport
}

\subsection{Precise nanothermal measurements via spa- tially distributed scanning thermal microscope}

\subsubsection{Introduction}

Over the last two decades scanning probe microscopy has become an indispensable tool supporting developments in the nanoscience and nanotechnology thanks to its atomic-scale spatial resolution and sensitivity to a wide variety of physical properties. In particular, scanning thermal microscopy (SThM) has enabled measurements of heat transport and temperatures at arbitrary selected points of the probed surface with lateral resolution down to a few nm. SThM's outstanding performance is largely due to a range of nanofabricated probes that are both sensitive and easy to use. From biological applications to active semiconductor devices, SThM is becoming the ultimate tool for probing thermal properties at the nanoscale $7,8,42,92,115$. Among the variety of thermal probes developed, the commercially available family of $\mathrm{Pd}$ resistive probes (PdRP) 45, 48 based on microfabricated SiNx cantilevers is one of the most extensively used amongst the SThM community. The heater of PdRP also functions as a temperature sensing element with its position close to the sample surface and a nanoscale sharp tip 
apex. Combined with a good stability over time, this probe is efficient for both temperature and thermal conductance mapping in contact or semi-contact modes. Moreover, its main advantage is its ability to work in a range of conditions from vacuum [34, 75] and air 49 to liquids 88$]$. Very significantly, its ability to perform relevant measurements of thermal transport in ambient conditions, that has been a limiting factor for other SThM probes[116], render PdRPs as the mainstream probe for nanothermal characterisation, they have been used extensively for a variety of applications from biological studies 117, 118 to soft matter 119 and condensed matter sciences $78,120,121]$.

However, merging the nanoscale resolution and high thermal sensitivity of a PdRP probe comes at a price - the heat flow through the nanoscale sized tip results in a temperature distribution which is detected by a thermal sensing element of several microns long. As a result, any analysis of sensor response must consider the heat transfer inside the spatially distributed heater and a highly heterogeneous probe, and corresponding sensor response have to be analysed in order to allow precise nanothermal measurements, and to, ultimately, enable fully quantitative measurements of nanoscale thermophysical parameters. Here we will: derive a concise model with a minimal number of parameters to correctly interpret the output of SThM measurements; compare these with experimental data of SThM operating in air and vacuum; and demonstrate the modification of the measurement setup allowing improvement of the stability, precision and quantitative interpretation of the measurements to be improved.

The typical PdRP sensor is composed of a $200 \mu \mathrm{m} \mathrm{Si}{ }_{3} \mathrm{~N}_{4}$ cantilever ending with a triangular beak-shaped tip of $14 \mu \mathrm{m}$ length. Two Pd lines $(\sim 1 \mu \mathrm{m}$ wide) are deposited on the sides of this triangular tip and joining at the tip apex. The sensor is included in a precision electrical circuit (usually a Wheatstone bridge) [50] which allows both the measurement of the probes resistance, which is linearly dependent on the temperature averaged over the sensor area, and to apply elevated currents (up to $\sim 1 \mathrm{~mA}$ ) for Joule heating the sensor and hence the probe. An example of 
the effect of finite dimensions of the heater can be seen on Fig. 3.1. which shows the Finite Element Analysis (FEA) of the probe temperature distribution due to Joule self-heating in the Pd lines. Fig. 3.1b shows the probe heated only by the contact with the heated sample. In both cases the temperatures of the tip apex are identical. For the self-heated probe (Fig. 3.1a) the temperature distribution along the length of the triangular tip is approximately linear. At the same time, for the heat flowing through the tip apex, the temperature distribution changes drastically (Fig. 3.1b) with temperature rising predominantly in the area around the heater apex. With the temperature sensing Pd lines extending along the whole length of the triangular tip, it is qualitatively clear that these temperature distributions will result in different probe resistance changes (and hence apparent probe temperatures) for the identical probe tip temperatures. The model developed here allows us to account for this difference.
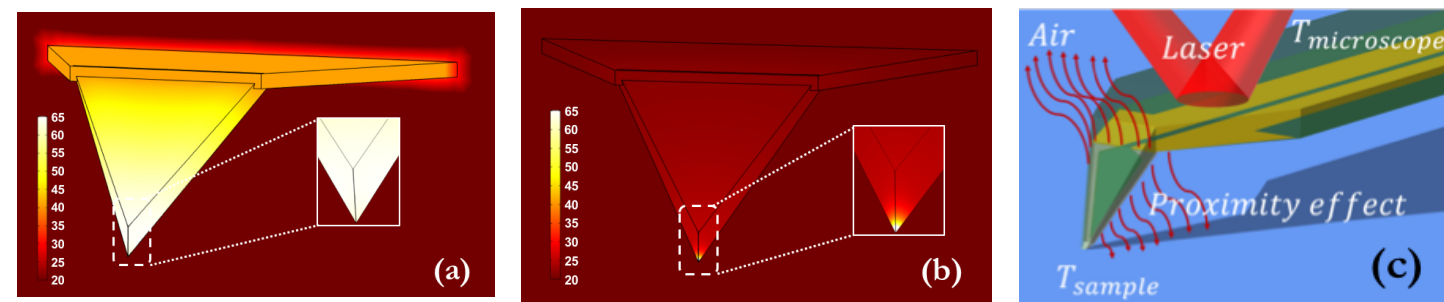

Figure 3.1: Finite Element Simulation of (a) the self-heated probe and (b) the sample-heated probe with the same temperature of $65 \mathrm{~K}$ (as represented by the color scale). (c) Schematic representation of the probe and the main sources of uncertainty.

Here we also consider other experimental parameters which can influence SThM measurements during the experiment namely variations in sample temperature and microscope temperature, affecting the temperature of the base of the sensor (Fig. 3.1c). These temperature variations - often referred to as "ambient temperature" - arise mainly from electrical effects arising from SThM operation, such as the feedback control of the position and laser illumination for positioning. In particular, the temperature variations due to laser illumination on the SThM probe [122 are difficult to quantify $[123$ as they vary depending on: i) the position of the laser beam on the cantilever which affects reproducibility of the measurements; ii) 
the laser source-cantilever distance which is not constant and thus causes variations of the probe temperature as it approaches the sample surface and iii) laser influenced artefacts arising from high topographical features and change of the sample reflectance. Indeed, a rise of $0.1 \mathrm{~K}$ was typically observed over a 30 minute timescale in our experiments as shown below. Such variations present a significant fraction of the probe temperature[48], and even small variations of either of those temperatures can cause significant loss in the measurements reproducibility.

In order to tackle these challenges, we modified our custom-made SThM setup with three elements:

- Monitoring of the sample and the probe mount (microscope) temperatures via high sensitivity calibrated thermistor temperature sensors to enable quantification of the thermal drift in the system and its correlation with the SThM signal variation.

- Active feedback stabilisation of those two temperatures using Peltier modules mounted under the sample and on the probe holder.

- For the single-point measurements, we incorporated a second step in the measurements procedure - firstly measurement with the laser illumination to obtain a force curve and secondly without the laser illumination for thermal characterisation.

\subsubsection{Combined analytical model and high-precision SThM setup}

\section{Analytical model of the SThM sensor}

To allow quantitative SThM measurements, we have built a model assuming a thermal resistance formalism consistent with the system's physics (see Fig. 3.2 a). The model is based on the regular triangular geometry of the probe and linear geometry of the $\mathrm{Pd}$ heater layer, as well as the environmental temperatures influencing the SThM measured signal. We solve the heat equation for the spatially 
distributed heater, with a finite number of input parameters, including power to the heater, temperatures of the sample, probe-holder and the temperature of air cell around the probe. The output of the model is the link between the tip-sample thermal resistance and "apparent" probe temperature measured via the change of the probe total electrical resistance, and its dependence on the input parameters. As the characteristic dimensions of the probe and sample often lie in the transitional regime between ballistic and diffusive heat transport [6], we have formulated measurements in terms of effective thermal resistances, similar to those reported in 124,125 . In Fig. 3.2 , the probe and tip are represented in green and the $\mathrm{Pd}$ film heater as two red strips. $T_{m}, T_{\text {air }}$ and $T_{s}$ are respectively microscope, ambient air and sample temperatures that act as the fixed-temperature heat-sinks for any heat flowing into the probe or generated in the probe heater, $Q_{h}$.

The finite dimensions of the $\mathrm{Pd}$ heater mean that the measured resistance is actually the electrical resistance averaged along the length of the heater. During the calibration procedure, the probe resistance $R_{e l}$ is linked to a calibration temperature $T_{c a l}$ by external uniform heating[50]. The relation established is the following: $R_{e l} \propto \hat{T}_{h}$ where $\hat{T}_{h}$ is the average heater temperature and $\hat{T}_{h}=T_{\text {cal }}$ in this case. For the self-heating, this average temperature differs from the temperature at the tip, $T_{t}$, due to the spatial distribution of the temperature over the heater dimensions (seen qualitatively in Fig. 3.1.). This gives rise to a temperature distribution $T(l)$ in the heater. This distribution should depend on the geometrical and physical specificities of the heater as well as on the environment conditions. 


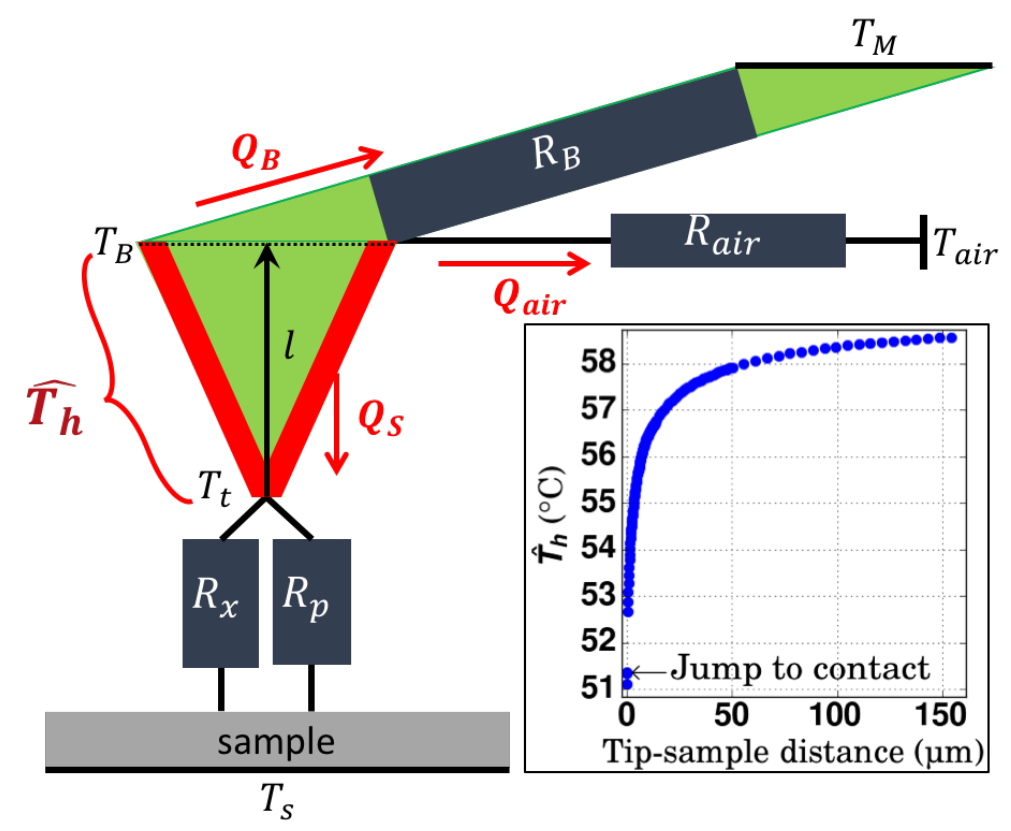

Figure 3.2: Thermal resistance network with spatially distributed heater and measured probe temperature as a function of tip-sample surface distance. Sample, microscope, and surrounding air heat baths are at temperatures $T_{S}, T_{M}$, and $T_{\text {air }}$, respectively. Heat produced $Q$ in the heater at the position $l$ is exchanged through 3 channels: $Q_{B}$ through the cantilever resistance $R_{B}$, Qair through the surrounding air resistance $R_{a} i r$, and $Q_{S}$ through the air gap proximity resistance $R_{p}$ and through the contact resistance $R_{x}$. The average heater temperature $\hat{T}_{h}$ is obtained by averaging the temperature at each position $l$ between the tip apex temperature $T_{t}$ and the temperature at the cantilever junction $T_{B}$

As the heater-sensor is distributed along the cantilever tip, the heat equation needs to describe a triangular system. First we consider the balance between the total heat generated in the heater and the different heat fluxes. Three heat channels are open: 1) through the cantilever base and its thermal resistance $R_{B}$, towards the microscope with temperature $\left.T_{M} ; 2\right)$ through $R_{\text {air }}$ to the ambient environment with temperature $\left.T_{\text {air }} ; 3\right)$ to the sample with temperature $T_{s}$ through both air proximity resistance $R_{p}$ and tip-sample contact resistance $R_{x}$ (where $R_{x}$ is only present when the direct contact between the tip and the surface is established). From the heat equation, we therefore can write

$$
Q_{h}=\frac{T_{B}}{R_{B A}}-\frac{T_{M}}{R_{B}}-\frac{T_{a i r}}{R_{a i r}}+\frac{T_{t}}{R_{x p}}-\frac{T_{s}}{R_{x p}}
$$

where $T_{B}$ is the temperature at the junction between the Pd heater and the can- 
tilever, $\frac{1}{R_{B A}}=\frac{1}{R_{B}}+\frac{1}{R_{a i r}}$ and $\frac{1}{R_{x p}}=\frac{1}{R_{p}}+\frac{1}{R_{x}}$.

It should be noted that the proximity air resistance is strongly dependent on the tip-surface distance in the range of 10-15 $\mu \mathrm{m}$ (as measurement shown in Fig. 3.2 inset) - the same order as the dimensions of the triangular part of the PdRP probe.

To see the main features of this model, we first consider the system in vacuum where $R_{a i r}=R_{p}=\infty$. The linear shape of the heater leads to the equal distribution of the Joule heat produced along the heater length. Due to the symmetry of the system, and the direction of the heat propagation along the axis of the probe, we can establish a link between $T_{B}$ and $T_{t}$ as

$$
T_{B}-T_{t}=a Q_{h}\left(\frac{\ln (1+b)}{b}-1\right)+\frac{a\left(T_{t}-T_{S}\right) \ln (1+b)}{R_{x p}}
$$

where $b=l_{h} / l_{t}$ and $l_{h}$ is the heater length and $l_{t}$ is the length of the truncated tip and $a=\frac{l_{h}+l_{t}}{2 h k r_{b}}$ where $h, k$ and $r_{b}$ are the heater thickness, effective thermal conductivity and half width at the cantilever junction, respectively.

From Eqs. 3.1 and 3.2 , we remove $T_{B}$ and obtain $T_{t}$. Then we can write the temperature distribution in the heater as:

$$
T_{h}(l)=T_{t}-\frac{a Q_{h}\left(l-l_{t}\right)}{b l_{t}}+a\left(\frac{Q_{h}}{b}+\frac{T_{t}-T_{s}}{R_{x p}}\right) \ln \frac{l}{l_{t}} .
$$

Eqs. 3.2 and 3.3 are obtained by integration of the heat equation along the direction $l$

Finally, in order to get the average heater temperature which is the parameter measured during experiment, we need to average this distribution over the heater length:

$$
\hat{T}_{h}=\frac{1}{l_{h}} \int_{l_{t}}^{l_{t}+l_{h}} T_{h}(l) d l
$$

Eq. 3.3 shows that the temperature distribution due to the self-heating is linear along the probe (second term in Eq. 3.3), whereas the temperature distribution in the probe due to the heat produced by the tip (third term in Eq. 3.3 has a non- 
linear logarithmic dependence. Together Eqs. 3.3 and 3.4 provide the temperature of the probe as the function of the probe-sample resistance. It is this resistance that is the ultimate parameter to be measured in SThM experiments.

\section{High precision SThM setup}

The ambient system was based on a Bruker (previously Veeco) Multimode head and Nanoscope III controller. Figs. 3.3 show the AFM/SThM setup. The main feature consists in adding a temperature control module. This module is made of:

- A Peltier plate below the sample.

- A temperature sensor reading this Peltier plate temperature (Pt resistor or thermistor) glued on the plate.

- A Peltier plate on the probe holder controlling the probe base temperature. Note the spring used both for keeping the Peltier in place and also for thermally connecting the Peltier to the surrounding.

- A temperature sensor for the second Peltier plate. This sensor is not glued directly on the Peltier but on the probe holder. This allows to monitor the temperature of the holder if the Peltier is not activated.

This module is controlled using a home-made LabView program which allows to control temperatures via feedback loops. 


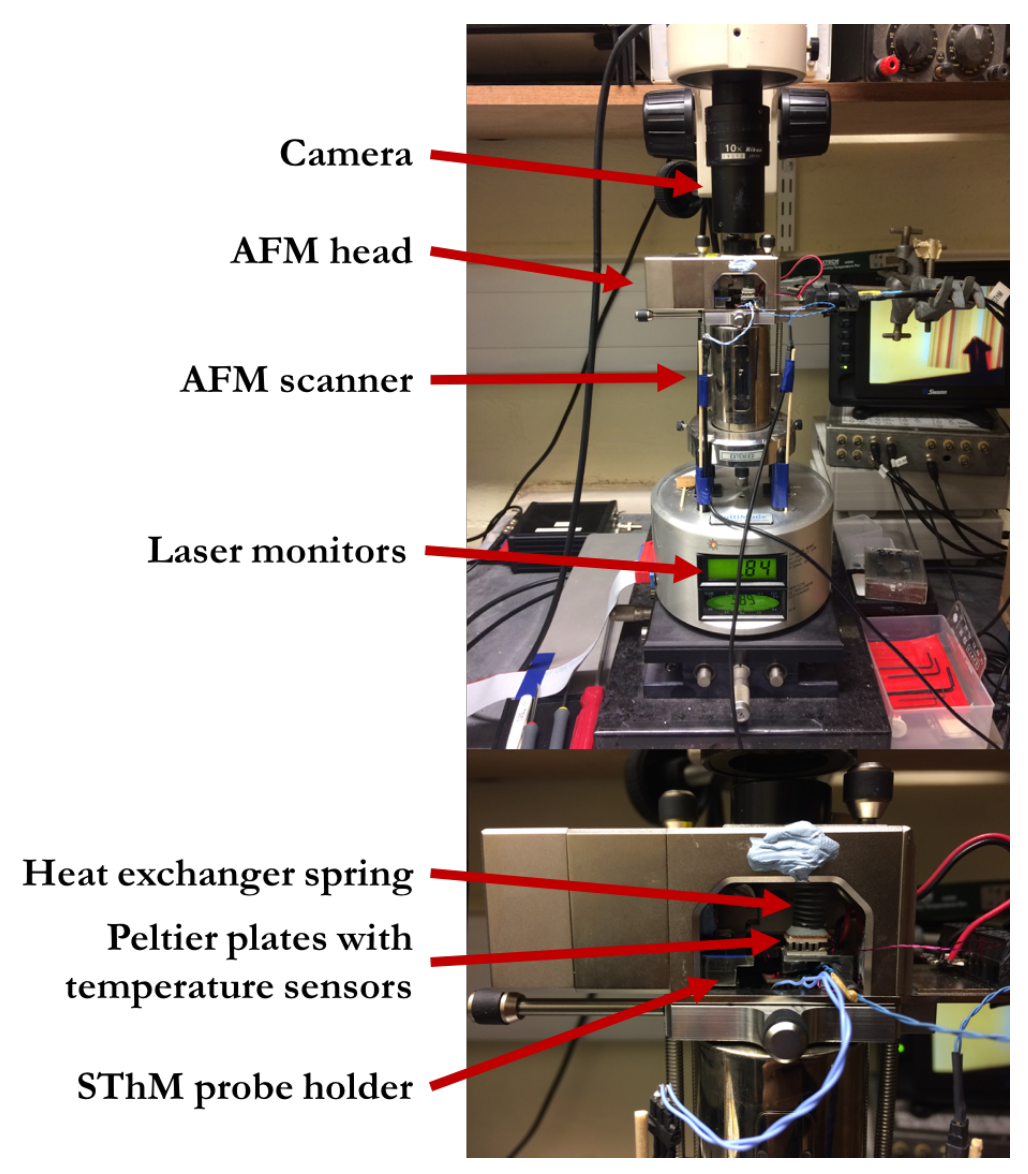

Figure 3.3: Ambient SThM setup with Peltier modules and sensors for temperature control.

In order to compare experimental data with our model, we first need to address several common sources of error that affect PdRP as well as any other SThM probes. We now report an experimental setup and measurement sequence which improves the stability and the precision of SThM measurement.

A set of typical electrical signals measured in an SThM are shown in Fig. 3.4 for ambient (a) and vacuum (b) conditions. The electrical SThM signal ( $V$ as a voltage) is proportional to the resistance of the self-heated probe and is recorded as the probe approaches to and then retracts from the sample surface. The SThM signal is directly proportional to the excess temperature of the heater wth respect to the ambient temperature $(V \propto T)$. When the probe snaps in and solid-solid contact is established, a sharp drop is observed linked with the increase of the heat transport through the $R_{x}$ channel (Fig. 3.2). During retraction, adhesion forces require an increased negative force to rupture the contact, hence the approach and 
retract curves do not overlap, creating hysteresis 126 . In air, adhesion forces are higher mainly due to the liquid meniscus; the increased hysteresis in air can be clearly observed when comparing air and vacuum curves. The voltage is recorded immediately prior to probe-sample contact $V_{n c}$ then as again in contact $V_{c}$. From these two voltages, we extract the SThM response $d V / V$ where $\frac{d V}{V}=\frac{V_{n c}-V_{c}}{V_{n c}}$.

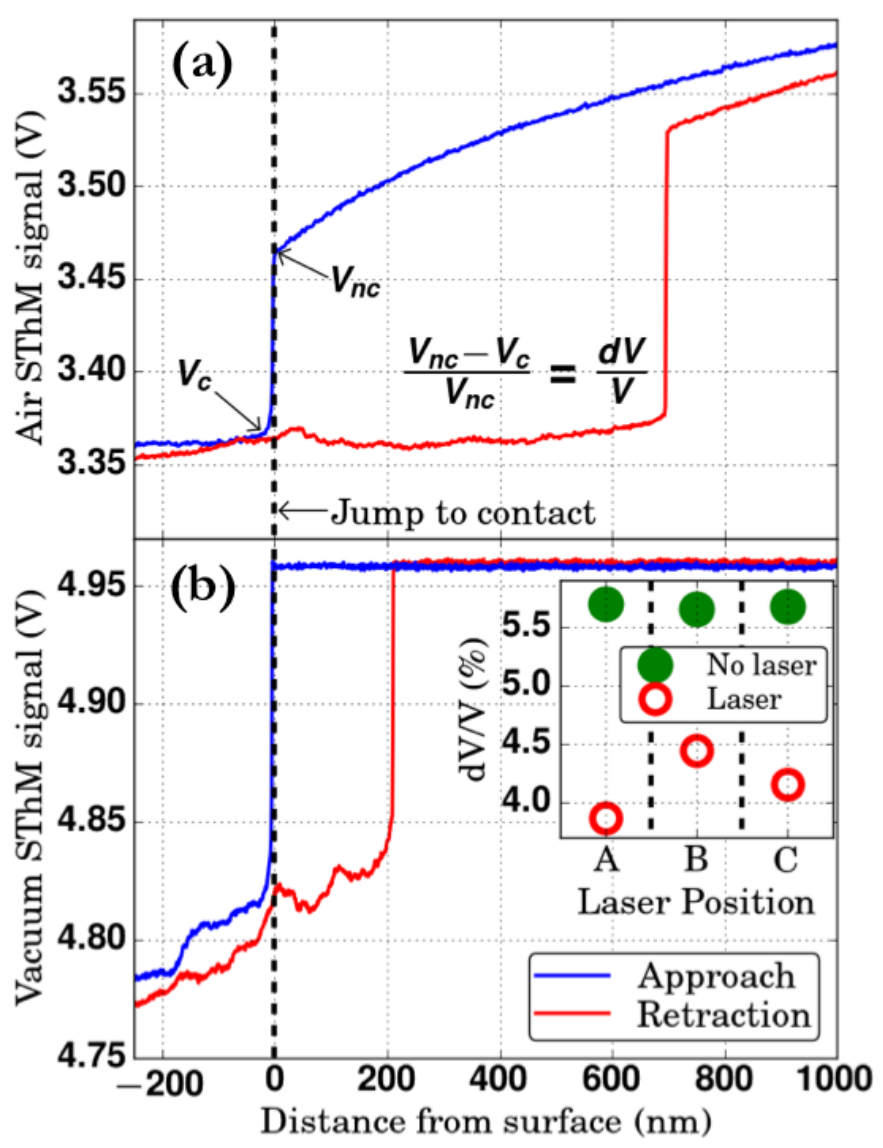

Figure 3.4: Approach (blue) - Retraction (red) SThM curves acquired with PdRP in ambient (a) and vacuum (b) conditions on a $\mathrm{SiO}_{2} / \mathrm{Si}$ sample. Inset: Thermal response $(d V / V)$ obtained when aligning the laser at 3 positions $(\mathrm{A}, \mathrm{B}, \mathrm{C})$ and compared to response without laser.

Whilst, from the model developed above, it can be shown that $d V / V$ depends most strongly on the tip-sample heat transfer, the laser illumination on the back of the cantilever can drastically affect the SThM signals (as mentioned above). The inset of Fig. 3.4 shows SThM responses for different laser positions on the probe with different laser alignments leading to a data scatter error of up to $30 \%$ of the absolute measured value. Additionally, the laser illumination can cause interference with the electrical signals affecting the measurement precision (see 
Fig. 3.5. Therefore, as initially observed by our group 122 and later confirmed elsewhere 123, conducting measurements in the absence of a laser can greatly improve the stability of SThM measurements. In all experiments reported below, the laser was shut off during thermal measurements.

In standard AFM Force-Spectroscopy, the laser deflection on the cantilever is related to the spring constant of both probe and sample. If the same procedure is applied with an SThM probe, both force and thermal signal can be recorded simultaneously as on Fig. 3.5 .

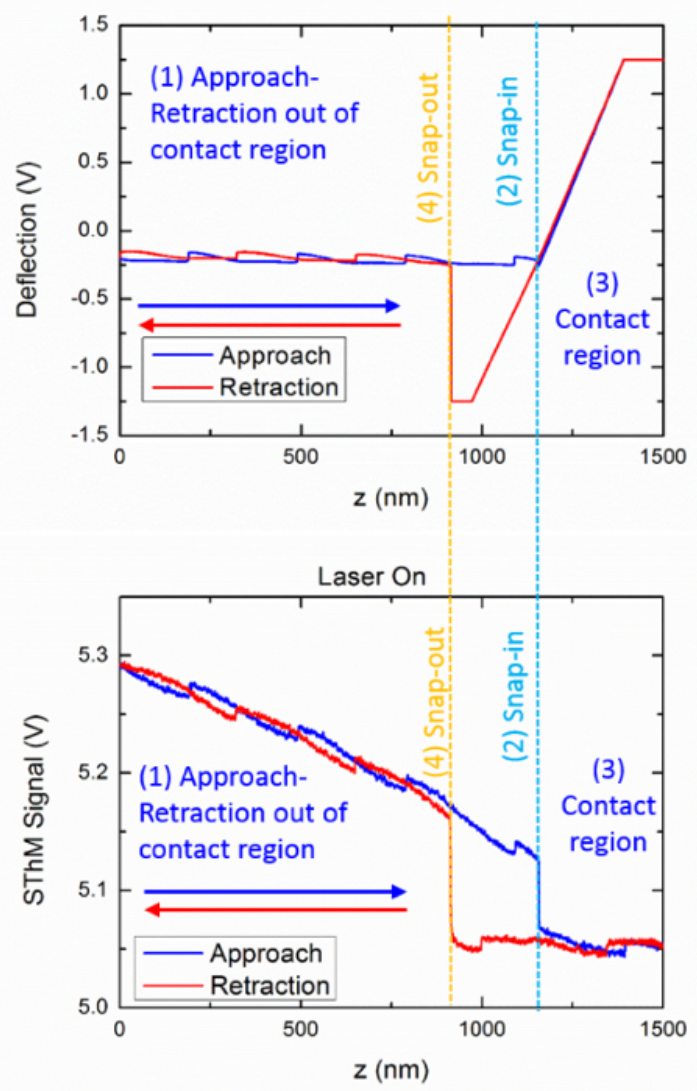

Figure 3.5: Approach (blue) - Retraction (red) force curve (top) andSThM curve (bottom) acquired simultaneously with PdRP in ambient conditions on a aluminium oxide sample. Note the periodic noise observed in both signals due to laser interferences.

Fig. 3.6 shows the effect of the sample and probe base temperatures on the reproducibility of the SThM response. We performed 240 consecutive approachretraction cycles on a $\mathrm{Si}$ sample at the same point while monitoring $T_{s}$ and $T_{M}$. The SThM signals, corresponding to $\hat{T}_{h}$ before (open circles, Fig. 3.6p) and after 
the contact (closed circles, Fig. 3.6b) were measured simultaneously with those of the sample (open circles, Fig. 3.6a) and microscope temperature (closed circles, Fig. 3.6a). One can observe that the thermal signals in- and out-of-contact are changing with time with a similar trend as the sample and microscope temperatures - confirming the model conclusion in Eq. 3.3 .

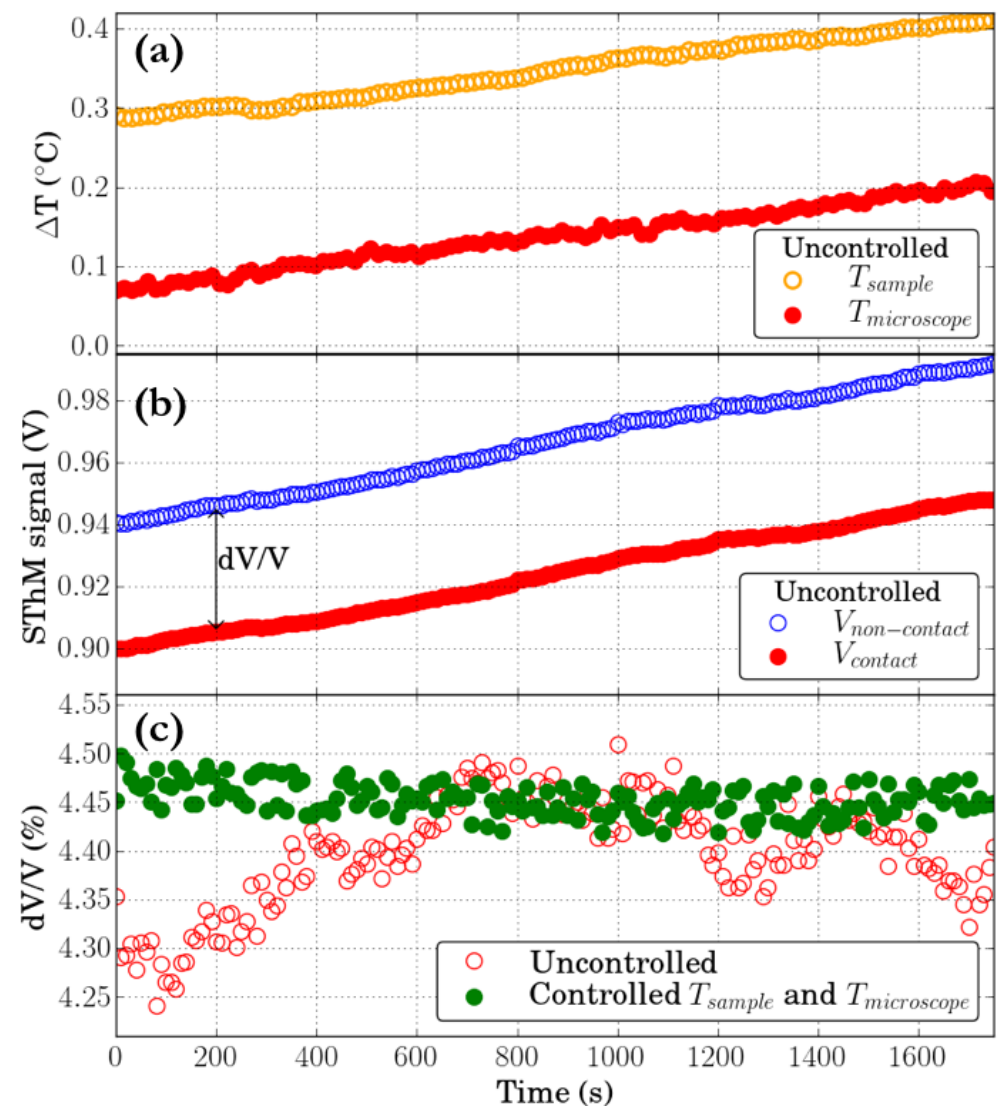

Figure 3.6: (a) Temperature of the microscope (red filled circles) and sample (yellow open cirles) vs time and (b) the corresponding measured SThM signal (excess probe temperature) (V) for tip out of contact (blue open circles) and in-contact (red filled circles) during consecutive point contacts on Si sample vs time. (c) Thermal response $(d V / V)$ acquired from consecutive point contacts at controlled (constant sample-microscope temperature) (green filled circles) and non-controlled (red open circles) sample temperature.

During an experiment, the thermal response is expected to be constant at the same point of a sample, when taking consecutive measurements. Instead, in the SThM setup with uncontrolled sample and probe base temperatures, we observe that the thermal response varied with time (see Fig. 3.6., open circles). To compensate for this temperature drift, we used Peltier plates mounted underneath 
the sample and on the probe holder, enabling a close loop control of temperatures $T_{s}, T_{M}$ via thermistors attached to the sample and the probe base. The repeated measurements in this setup (Fig. 3.6. filled green points) showed about 5-10 fold stability increase over the same measurement time. These results indicate that keeping the sample and probe holder temperatures constant leads to significantly more reproducible SThM measurements.

\subsubsection{Results and discussion}

\section{Experimentally measured model parameters}

In order to measure accurately the main thermal resistances of the model, a specific procedure needs to be followed. First, we place the probe in vacuum. In this case, $\hat{T}_{h}$ is given by

$$
\hat{T}_{h}=\left(R_{B}+\gamma_{s h}\right) Q_{h}+T_{m}
$$

where $\gamma_{s h}=\frac{l_{h}+l_{t}}{2 h k r_{b} l_{h}}\left[\frac{l_{h}}{2}+\frac{l_{t}^{2}}{l_{h}} \ln \left(\frac{l_{t}+l_{h}}{l_{t}}\right)-l_{t}\right]$. Then, knowing the power $Q_{h}$ injected in the probe and the excess temperature $\hat{T}_{h}-T_{m}$, we can measure the probe total thermal resistance $R_{B}+\gamma_{s h}$. The contribution of the triangular tip with finite dimensions of the heater is often neglected in the literature $127 \cdot \sqrt{129}$ and values reported for the probe thermal resistance just take into account $\gamma_{s h}$. Our approach gives therefore a more precise understanding of the heat transfer within the probe.

Decoupling $R_{B}$ from $\gamma_{s h}$ is difficult experimentally. However, Finite Element analysis previously reported[50] or an analytical model such as the one proposed by Ge et al. 130 , show that $R_{B}$ accounts for around 25 to $50 \%$ of the total thermal resistance measured and $\gamma_{s h}$ for the rest. We measured the total probe thermal resistance to be $R_{B}+\gamma_{s h}=7 \pm 0.5 \times 10^{4} \mathrm{KW}^{-1}$ which gives $R_{B}=1.8 \pm 0.1 \times 10^{4}$ $\mathrm{KW}^{-1}$ and $\gamma_{s h}=5.3 \pm 0.4 \times 10^{4} \mathrm{KW}^{-1}$ assuming $\frac{R_{B}}{R_{B}+\gamma_{s h}}=0.25$.

In air and far away from the sample surface, we have

$$
\hat{T}_{h}=T_{m} \frac{R_{\text {air }}}{R_{B}+R_{\text {air }}}+T_{\text {air }} \frac{R_{B}}{R_{B}+R_{\text {air }}}+Q_{h}\left(\gamma_{s h}+\frac{R_{B} R_{\text {air }}}{R_{B}+R_{\text {air }}}\right)
$$


As we deduced $R_{B}$ and $\gamma_{s h}$ from the previous step, we now can obtain $R_{a i r}$ if we know $T_{\text {air }}$. We obtained $R_{\text {air }}=5 \pm 1 \times 10^{5} \mathrm{KW}^{-1}$.

Finally, if we move the probe toward the sample surface, we notice a drop in the probe average excess temperature (see Fig. 3.2). This arises from the heat conductance through the air gap "proximity" which we called $R_{p}$. Comparing the signal far away from the surface to the one just before snap-in, we can measure $R_{p}$ for a given power $Q_{h}$. As we will discuss in the last section, the proximity effect plays a strong role in measurements performed in air. Measured values for the model parameters $R_{B}, \gamma_{s h}, R_{a i r}$ and $R_{p}$ are given in the Table 3.1.

The final expression for the average heater temperature in air and in contact with the sample is given by:

$$
\hat{T}_{h}=\alpha Q_{h}+\beta T_{m}+\gamma T_{s}+\delta T_{a i r}
$$

where coefficients $\alpha, \beta, \gamma$ and $\delta$ are functions of $R_{B}, R_{a i r}, R_{p}, R_{x}$ and $l_{h}, l_{t}$ which are probe physical and geometrical parameters. These coefficients depend on the probe conditions, namely whether the probe is in contact with the surface or not.

The key consequence of the model summarised in Eq. 3.7 is that it links the key value measured in SThM ("measurand") $\hat{T}_{h}$ with environmental temperatures $T_{m}, T_{s}, T_{a i r}$ and heat (Joule heating in the probe) $Q_{h}$. The proposed calibration protocol - air resistance calibration, vacuum $\alpha, \beta, \gamma$ calibration and air $\delta$ calibration - allow the full quantification of all properties of a particular probe.

\begin{tabular}{|c|c|}
\hline Parameter & Measured value $\left(\mathrm{KW}^{-1}\right)$ \\
\hline$R_{B}$ & $1.75 \pm 0.08 \times 10^{4}$ \\
$\gamma_{s h}$ & $5.25 \pm 0.08 \times 10^{4}$ \\
$R_{a i r}$ & $5 \pm 1 \times 10^{5}$ \\
$R_{p}$ & $7 \pm 1 \times 10^{5}$ \\
\hline
\end{tabular}

Table 3.1: Measured nominal values of the model parameters.

The essential feature of the model developed above is that it accounts for the 
probe geometry. As the heater is distributed on the triangular tip, a temperature gradient is inevitably formed. This gradient is different in and out of contact. It is also different depending on whether the heat is generated within the probe (self-heating case) or is coming from the sample through the tip. In Fig. 3.7a, temperature distributions are displayed for self-heating of the probe and for heat flowing from the sample. One can see that the distributions are drastically different. Note that in Fig. 3.7 a we compared Finite Element modelling to our analytical model (Eq. 3.3) and found a similar trend which supports the analytical model presented.

Furthermore, a key finding of our model is the comparison of heat fluxes to the self-heated probe and to the sample. When power is provided to the heater (via the $\mathrm{Pd}$ film), the excess temperature rises with $\frac{d \hat{T}_{h}}{d Q_{h}}=20.5 \pm 0.5 \mathrm{Km}^{-1} \mathrm{~W}^{-1}$. However, when the same amount of heat is sent through the tip apex, the excess temperature is higher than the self-heating one. In this case, the excess temperature rises with $\frac{d \hat{T_{h}}}{d Q_{s}}=31.9 \pm 0.5 \mathrm{Km}^{-1} \mathrm{~W}^{-1}$. This fundamental difference of more than $50 \%$, is often neglected in the interpretation of SThM results. Thus, a model taking into account the heater dimensions allows to correct a major parameter of the probe and significantly improves the measurement accuracy. 

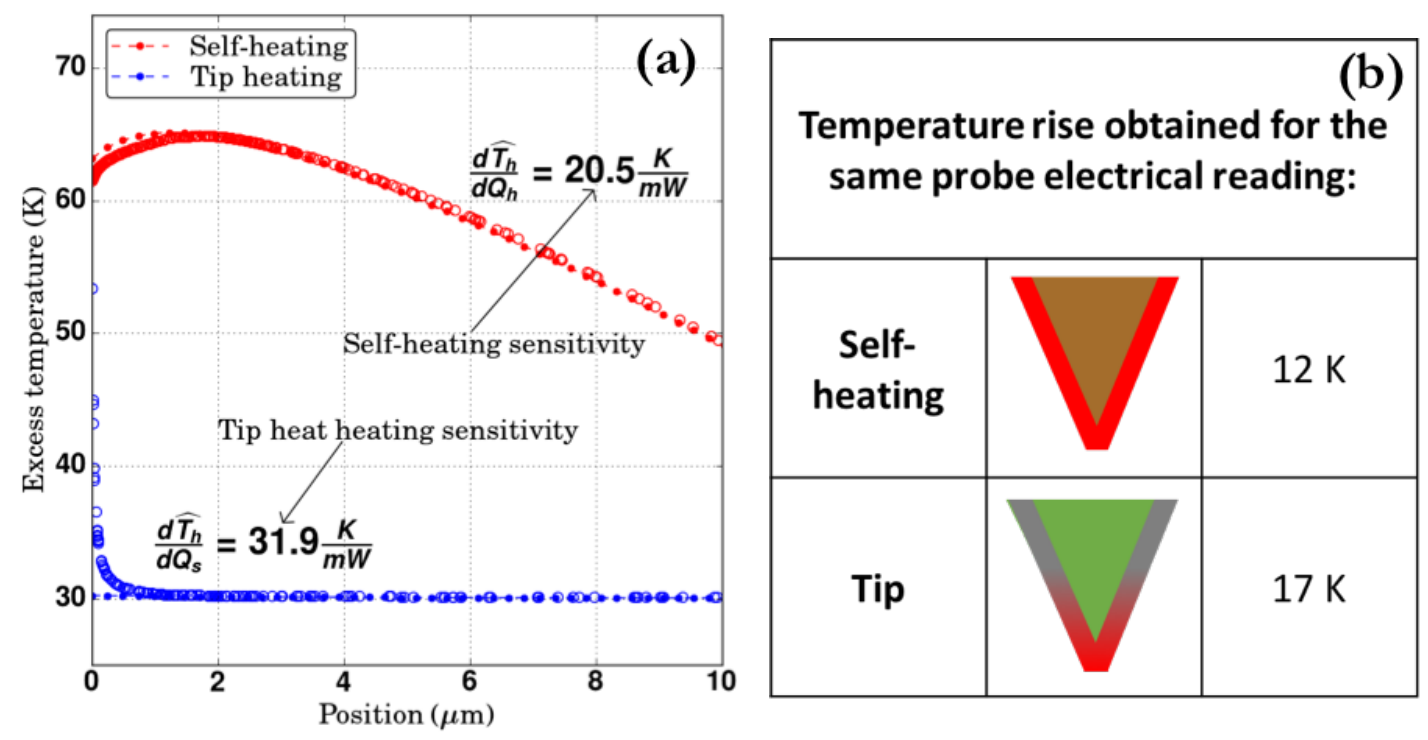

Figure 3.7: (a) Heater temperature distribution for the self-heated case (triangles) and heating through the tip (circles) as obtained from finite element modelling (open circles and triangles) and analytical model (filled circles and triangles). (b) Values of computed temperature rise obtained for the same electrical reading: color of the heater at the edge of the probe schematically represents temperature distribution in the probe. The two temperatures are often considered to be the same.

As we mentioned earlier, in the non-contact case and far away from the sample, the tip temperature $T_{t}$ is always higher than the average heater temperature $\hat{T}_{h}$. In Fig. 3.7b, we compute and compare these two temperatures. For the same electrical reading, e.g. electrical resistance, two different temperatures are obtained. Full curves are shown in Figure 3.8. Importantly, self-heating and tip temperature differ by more than $40 \%$. This model result highlights the importance of taking into account the heater dimensions in order to extract physical quantities of the SThM measurement. 


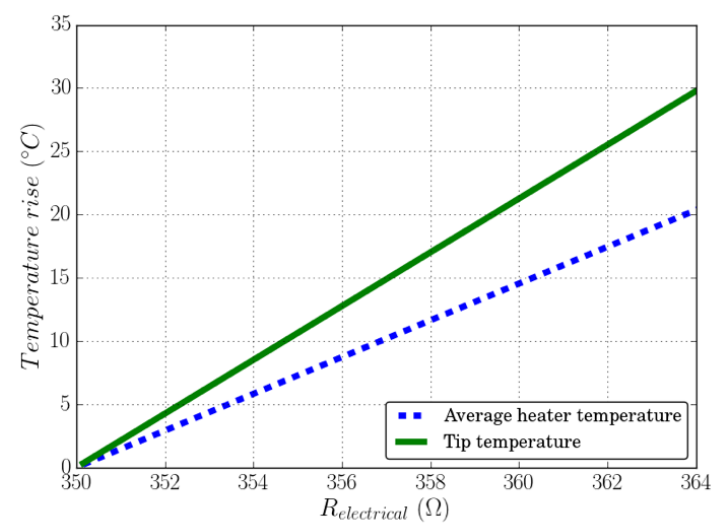

Figure 3.8: Modelled temperature rise dependence on the electrical resistance for the average heater temperature (dotted blue) and the tip temperature (green line).

\section{Model outputs vs multiparametric experiments}

We performed experiments in different conditions in order to compare our model with experimental data. A set of SThM measurements on a single crystalline diamond sample (Element Six, UK) were realised. Diamond was chosen for its high thermal conductivity ( $>1000 \mathrm{Wm}^{-1} \mathrm{~K}^{-1}$ ) and therefore the main component of the thermal response will be the contact resistance at the probe apex leading to minimal effect of the sample itself. The SThM response $d V / V$ for different powers was measured for 3 sample temperatures from below to above microscope temperature. This experiment was performed both in vacuum and in air environments. Model parameters were then changed to correspond to experimental conditions. The outputs of measurements and model were subsequently compared (Figures 3.9 .

As we see at Fig. 3.9a, a linear trend is obtained in vacuum for both model and experiment. Model results are plotted in solid lines. Noticeably, when the microscope and sample temperatures are equal, the SThM response does not depend on the power injected in the heater. Such measurement conditions (vacuum and equal sample and microscope temperatures) provide the best system for precise and repeatable SThM measurements.

In air, the situation differs drastically (see Fig. 3.9p). The proximity effect 
is playing a key role through the air gap resistance between the heater and the sample surface. To account for this effect, we changed the value of the air gap resistance for the different powers. It is likely that different excess temperatures of the probe would lead to different proximity resistances. We assumed an inversely proportional air gap resistance with input power. As we can see in Fig. 3.9p, when microscope and sample temperatures are equal, the SThM response for different probe temperature deviates by $0.5 \%$. Finally, we also notice that our model does not account fully for this proximity effect as such non-linear effects observed on the experimental data could not be reproduced numerically. This result highlights the importance of the proximity effect in ambient SThM measurements.
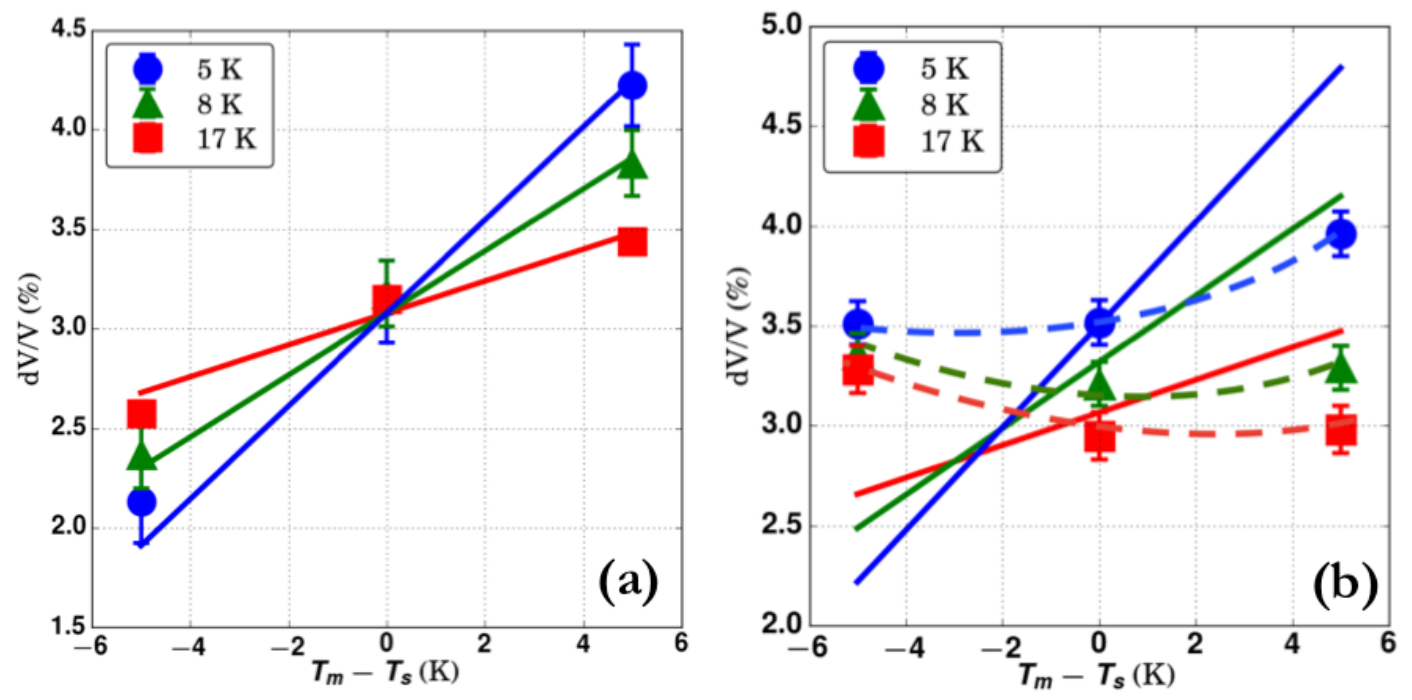

Figure 3.9: Experimental (solid circles) and model (solid lines) results for the thermal response $d V / V$ as a function of sample temperature in vacuum (left) and in air (right) for three average heater excess temeprature (5K, $8 \mathrm{~K}$ and $17 \mathrm{~K})$. Dashed lines in panel (b) are a guide for the eye.

\subsubsection{Summary}

This section explored a sensitivity of SThM based on the most widely used commercial metal resistive probe capable to operate in air, vacuum and liquid environments. By building a simple semi-analytical model, we demonstrated the importance of taking into account the finite dimensions of the SThM sensor. The model and experiments show that, with the same amount of heat, the probe selfheating and heating through the tip result in different probe response as large as 
$50 \%$ for a typical probe. Furthermore, crucial parameters impacting the experimental results were found - namely the influence of the laser providing position feedback in typical SThM setup, and small variations of the sample and microscope temperatures, often assumed to be equal, were shown to significantly affect the measurements reproducibility and precision.

By adding Peltier temperature control of the sample and the probe holder, we substantially decreased data scatter from $5.5 \%$ to $0.5 \%$. Allowing two step measurements - first with a laser to monitor the sample topography and then blocking the laser for SThM measurements - further improved the SThM reproducibility as the laser was found to add up to $30 \%$ to the data scatter. Overall, we obtained good agreement between our model and experiments when the system is under vacuum. In air, we could partially reproduce the experiment by changing the proximity thermal resistance. This result shows the importance of the proximity effect in scanning thermal microscopy measurements which requires more precise modelling.

Both the modelling and experimental aspects of this study allow a significant improvement in precision of current scanning thermal microscopy techniques. The increased precision and interpretation of the SThM measurements may provide significant insight into the mainstream semiconductor sector looking for nanoscale heat dissipation in advanced processors, novel nanostructured thermoelectrics and thermal interface materials.

\subsection{Introducing cryoSThM - nanothermal trans- port measurements at cryogenic temperatures}

The model presented above demonstrated the crucial impact of the ambient air on the experimental outputs. Also the impact of the probe sensor geometry was highlighted. In this section, we demonstrate SThM measurements at cryogenic temperatures. As cryogenic environment benefits from vacuum conditions, we 
use a simpler system working in vacuum environment and with a doped silicon probe. Temperature dependent studies are of great interest as thermal properties are likely to vary with temperature. Promises of new thermal phenomena drove the development of this cryoSThM.

Here we derive the impact on the sample temperature on the SThM response in the first part and we show how the thermal resistance between the sensor and the sample heat sink is linked to the SThM response. In the second part, we demonstrate this model on bulk materials of known properties and discuss the different effects of the sample temperature on the thermal resistance measured.

\subsubsection{Vacuum SThM setup for measurements at cryogenic temperatures}

SThM measurements performed in vacuum can be realised with the doped silicon probe providing better spatial resolution and increased sensitivity to high thermal conductivity materials 50 . Turbomolecular and ions pumps ensure high quality vacuum while the SThM microscope is suspended on a magnetic damping system for minimal mechanical noise (see Fig. 3.10a). 


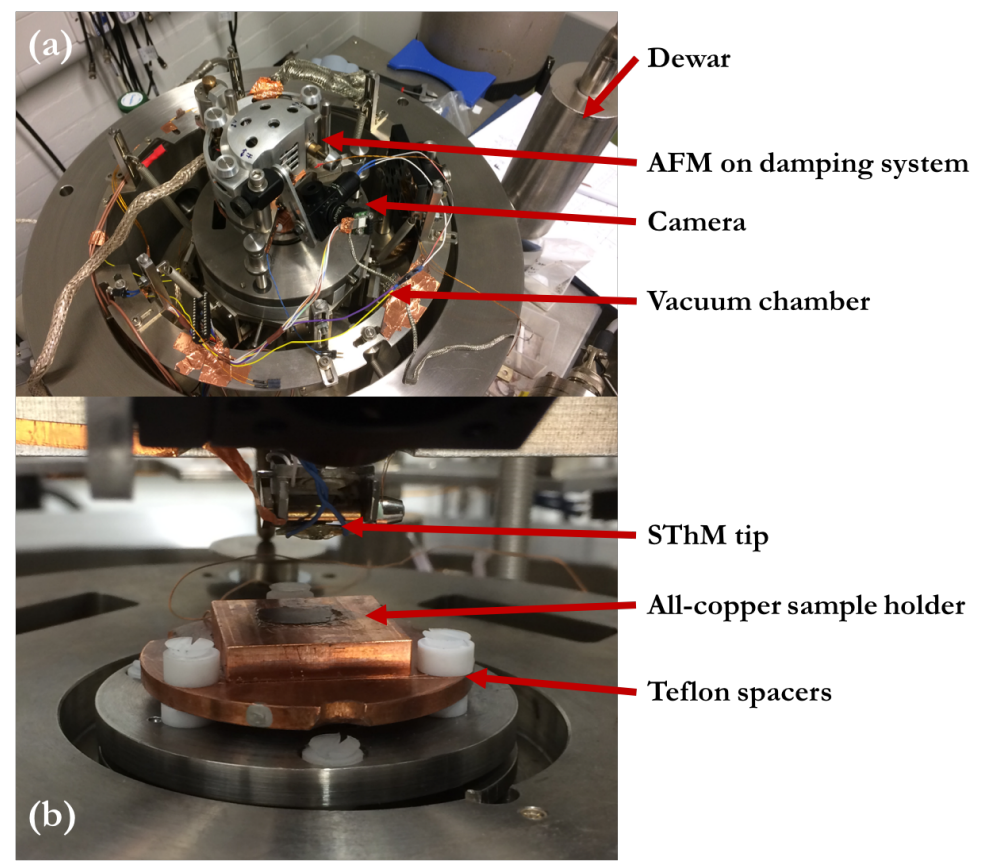

Figure 3.10: (a) Vacuum SThM chamber with cooling capabilities. (b) cryoSThM sample holder thermally insulated from the surrounding via Teflon spacers.

As we see in Fig. 3.10a, a liquid nitrogen dewar is connected to the vacuum chamber. This dewar connects to a copper braid that is thermally linked to the sample holder. Personal Protection Equipment (PPE) must be worn when manipulating the liquid nitrogen.

When the sample is cooled down, the pressure will be greatly reduced compared to ambient temperature pressure. This is due to the braid acting as a cryo-pump. In such a case, the turbomolecular pump can be switched off with the ion pump keeping the vacuum. That will further reduce mechanical noise on the SThM images.

Fig. $3.10 \mathrm{p}$ shows the sample holder for low temperatures measurements. This all copper holder is connected to the copper braid and therefore linked to the liquid nitrogen dewar. In order to thermally separate it from the microscope stage system, plastic screws and spacers are used. The temperature on the sample holder is obtained from the calibrated resistance measurement of a Pt resistor and read through a LabView program. Due to the several components and connections between the liquid nitrogen and the sample stage, the lowest temperature ever 
recorded at our lab was $140 \mathrm{~K}$. Surface mounted resistors were placed below the sample stage and used as controllable heaters to stabilise the sample temperature.

Low temperature SThM measurements have to be performed in good quality vacuum to avoid contamination of the sample surface. As the braid and sample are cooling down, they act as a cryo-pump and any residual moisture or contamination will condensate on the cold surfaces. Therefore, it is important to ensure that the pressure is below $1 \times 10^{-6}$ Torr and in the best case, that the vacuum chamber is clean. This can be improved by baking the whole system regularly.

CryoSThM experiments are usually performed as follows:

- Prepare the system and sample, and pump the chamber down until $1 \times 10^{-6}$ Torr is reached.

- Perform room temperature measurements on the sample that will be used as control.

- Pour liquid nitrogen in the dewar. Keep the sample at room temperature while the braid is cooling down to avoid the sample acting as a cryo-pump.

- Perform low temperature experiments and use surface a mounted resistor to gradually increase the sample holder temperature if desired.

- Finally, perform room temperature measurements again to control repeatability of the experiment.

\subsubsection{Measurement model}

As we pointed out in the literature review, the doped silicon probe sensor can be represented by a simple point heater model without considering its geometrical dimensions $[70 \mid$. This is due to the relative size and position of the heater located at the end of two silicon legs and on top of a triangular tip. When the probe is under an applied power load (e.g. $\sim 1.2 \mathrm{~mW}$ ), it gives rise to an excess temperature (e.g. $\Delta T \sim 100 \mathrm{~K})$ with respect the probe base temperature. This excess temperature 
then relates to the SThM output voltage, $V$, by a calibration factor $\alpha$ :

$$
\Delta T=\alpha V
$$

When out-of-contact, the only heat transfer channel is through the probe and cantilever themselves. We have $\Delta T_{n c}=\alpha V_{n c}=Q \times R_{p}$ where $R_{p}$ is the thermal resistance of the cantilever. Knowing the excess temperature and total power $Q$, we measure $R_{p}=5.4 \pm 0.1 \times 10^{4} \mathrm{KW}^{-1}$.

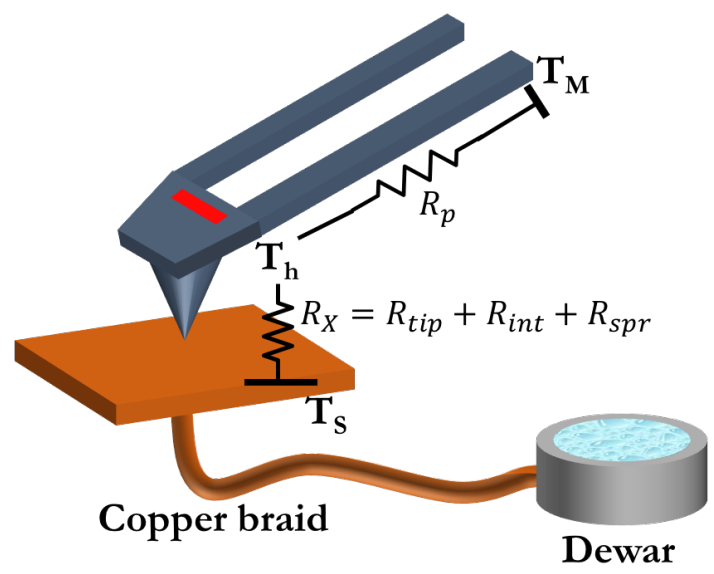

Figure 3.11: Schematic diagram of the cryoSThM system in vacuum. The sample holder is connected to a Dewar via a copper braid. The probe heater is at the temperature $T_{h}$ thermally connected to the microscope at temperature $T_{M}$ through the probe thermal resistance $R_{p}$ and to the sample at temperature $T_{S}$ through $R_{X}$ that is made of the conical tip thermal resistance $R_{\text {tip }}$; the interface resistance $R_{\text {int }}$ and the spreading resistance of the sample $R_{s p r}$.

When the probe is brought into contact with a sample, a new heat transfer channel is open going from the heater to the sample heat sink. If both the probe base and the sample heat sink are at the same temperature then we can write:

$$
\Delta T_{c}=Q \times R_{e q}=Q \times \frac{R_{p} R_{X}}{R_{p}+R_{X}}
$$

where $R_{e q}=\frac{R_{p} R_{X}}{R_{p}+R_{X}}$ and where $R_{X}$ is the thermal resistance of the opened heat transfer channel. This thermal resistance is made of several components in series. First, the constriction resistance of the sharp conical tip $R_{t i p}$. Then, the interface resistance between the tip apex and the sample surface $R_{\text {int }}$. Finally, the spreading 
resistance of the sample $R_{s p r}$ which might be made of several components. Finding $R_{X}$ is the last step of the experimental measurement. From there, a model is always needed.

By bringing the probe in and out of contact while its temperature is recorded, we obtain the normalized temperature change between out and in contact:

$$
\frac{\Delta T_{n c}-\Delta T_{c}}{\Delta T_{n c}}=\frac{V_{n c}-V_{c}}{V_{n c}}=\frac{R_{p}-R_{e q}}{R_{p}}=\frac{R_{p}}{R_{p}+R_{X}} .
$$

As we can see, the SThM response $d V / V$ depends only on the probe thermal resistance and the measured thermal resistance which can then be obtained directly.

This general introduction assumed that all heat sinks where at the same temperature: $T_{M}=T_{S}=T_{0}$. In the general case (see Fig. 3.11), this might not always be true, especially when dealing with cryogenic systems. In such a system we have the following relation for the heat conservation:

$$
Q_{h}=Q_{B}+Q_{S}
$$

where $Q_{h}$ is the total heat generated in the probe heater and where $Q_{B}$ and $Q_{S}$ are heats going to the probe base and sample heat sinks respectively.

If we call $T_{h}^{n c}$ and $T_{h}^{c}$, the absolute heater temperature out of- and in-contact with the sample, respectively, we can write for the non-contact and in-contact cases:

$$
Q_{h}=\frac{T_{h}^{n c}-T_{M}}{R_{p}} \quad \text { and } \quad Q_{h}=\frac{T_{h}^{c}-T_{M}}{R_{p}}+\frac{T_{h}^{c}-T_{S}}{R_{X}} .
$$

By rearranging, we find the heater temperature out and in contact:

$$
T_{h}^{n c}=R_{p} Q_{h}+T_{M} T_{h}^{c}=\left(\frac{R_{p} R_{X}}{R_{p}+R_{X}}\right)\left(Q_{h}+\frac{T_{M}}{R_{p}}+\frac{T_{S}}{R_{X}}\right)
$$

We are not measuring absolute temperatures but only excess temperatures with respect to the temperature at which the bridge balance was realized. This temperature is $T_{M}$. So for both in and out contact we have: $T_{h}^{c, n c}=\alpha V_{c, n c}+T_{M}$ 
We can now write the equivalent expression as Eq. 3.10 taking into account the sample and microscope temperatures:

$$
\frac{V_{n c}-V_{c}}{V_{n c}}=\frac{T_{h}^{n c}-T_{M}-T_{h}^{c}+T_{M}}{T_{h}^{n c}-T_{M}}=\frac{1}{R_{p}+R_{X}}\left(R_{p}+\frac{T_{M}-T_{S}}{Q_{h}}\right)
$$

or rearranging for $R_{X}$ :

$$
R_{X}=\frac{V_{n c}}{V_{n c}-V_{c}}\left(R_{p}+\frac{T_{M}-T_{S}}{Q_{h}}\right)-R_{p}
$$

As we can see, if $T_{M}=T_{S}$, Eq. 3.15 simplifies to Eq. 3.10. Otherwise, the temperature difference between the microscope and the sample has to be taken into account in the measured thermal resistance.

\subsubsection{CryoSThM demonstration}

To demonstrate the cryo-SThM and assess the temperature effect on the measurements, we used two bulk samples with known thermal properties: a $280 \mathrm{~nm}$ silicon oxide film on silicon and a silicon wafer. Silicon oxide thermal conductivity is reducing with decreasing temperature 131 whereas silicon thermal conductivity first increases when temperature decreases, up to $T_{\text {sample }} \approx 30 \mathrm{~K}$ where it starts decreasing 132. As we only reach a minimum of $140 \mathrm{~K}$, we consider that silicon thermal conductivity only increases with reducing temperature. Note that this increasing conductivity arise from a general increase of the phonon mean free path. In fact, as temperature decreases, the Umklapp and isotope scattering length increase drastically[133] therefore increasing the phonon mean free path. This will play a crucial role as we will observe.

We performed measurements of the SThM response $d V / V$ for silicon oxide and silicon in the range of 140 to $300 \mathrm{~K}$. These materials were chosen for their different thermal conductivity behaviour with temperature. Using the model derived above, we obtained the thermal resistance measured between the heater and the sample heat sink. Results are shown in Fig. 3.12. For silicon oxide, we observe a general 
increase with decreasing temperature while for silicon, first resistance increases and then decreases after a small plateau between 170 and $220 \mathrm{~K}$. In order to correctly interpret these results, it is important to remember what components create this thermal resistance. Between the heater and the sample heat sink there are three main thermal resistances: the conical tip resistance, $R_{t i p}$, the interface between the tip and the sample, $R_{\text {int }}$, and the spreading resistance within the sample, $R_{\text {spr }}$ :

$$
R_{X}=R_{t i p}+R_{\text {int }}+R_{\text {spr }}
$$
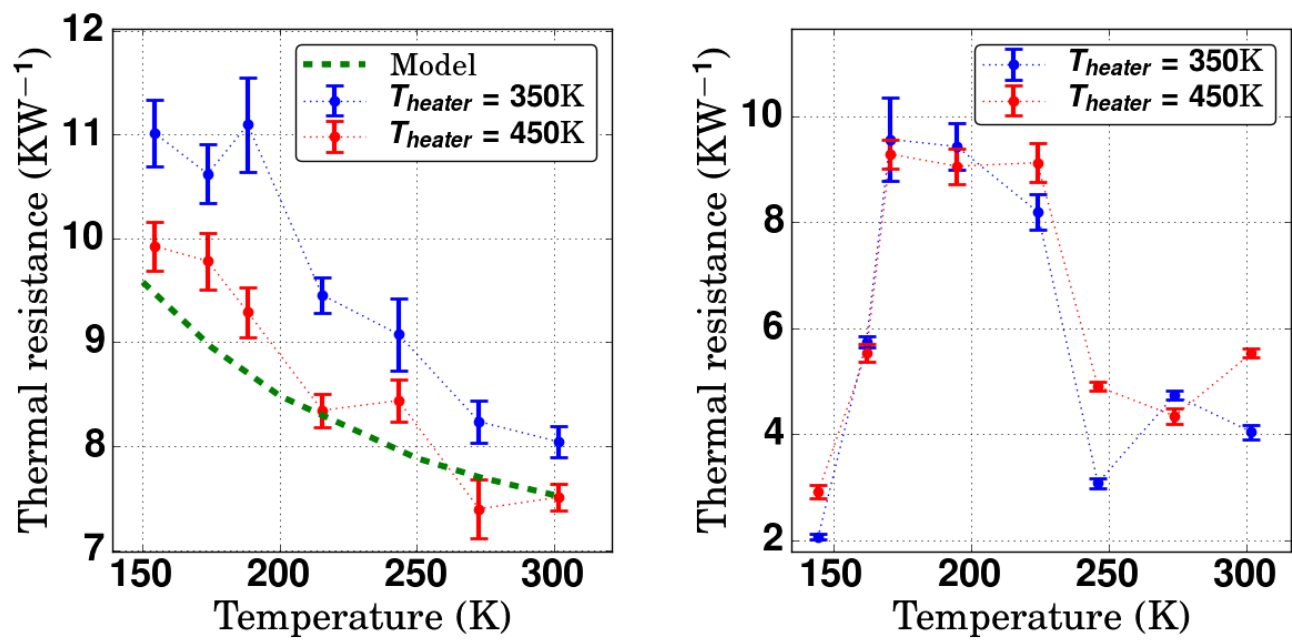

Figure 3.12: Measured thermal resistance in contact with $\mathrm{SiO}_{2}$ (left) and $\mathrm{Si}$ (right) at various sample temperatures with two probe heater temperatures at $350 \mathrm{~K}$ (blue) and 450K (red). The measurement on silicon oxide can be modelled by a standard diffusive model (green dotted line) but the silicon results require more interpretation. For silicon oxide, the two heater temperatures do not coincide highlighting that the hot probe heats the sample and therefore changes the thermal conductivity locally. For silicon, this is not observed as silicon as a much higher thermal conductivity.

Given the last two components of the measured thermal resistance, it is difficult to decouple the effect of one or the other on the SThM signal change. The spreading resistance in a bulk system is inversely proportional to thermal conductivity $k\left(R_{s p r}=1 / 4 k a\right.$ with $a$ the contact radius). This is observed on the silicon oxide where resistance increases with reducing temperature due to the decreasing silicon oxide thermal conductivity. We plotted $R_{t i p}+R_{\text {int }}+1 / 4 k_{S i O x}(T) a$ using temperature dependent thermal conductivity values from literature 131. Esti- 
mating the contact radius $a=40 \mathrm{~nm}$ from SEM imaging (see Appendices) and assuming $R_{t i p}+R_{\text {int }}=3.5 \times 10^{6} \mathrm{KW}^{-1}$, the measured thermal resistance variation corresponds well to the change of temperature dependent thermal conductivity, as observed on Fig. 3.12 .

For silicon, the results obtained require more interpretation. The first increase in the measured resistance can be attributed to the increasing phonon mean free path. As the tip radius was measured to be around $40 \mathrm{~nm}$ by SEM, it is much lower than the bulk phonon mean free path of silicon[133]. Furthermore, as we mentioned earlier, when temperature reduces, scattering lengths increase and thus the mean free paths become larger[15]. In silicon, the average mean free path at room temperature is around $300 \mathrm{~nm}$ while at $150 \mathrm{~K}$ it increases to around $1 \mu \mathrm{m}$. The $40 \mathrm{~nm}$ radius heat source is then smaller than these mean free path values and ballistic effects need to be accounted for.

Following Prasher 27 model for a nanoscale constriction, a nanoscale heat source will experience a ballistic resistance at the contact when the mean free path is comparable or bigger than the heat source dimensions. Diffusive and ballistic components of the constriction resistance can be added to obtain a realistic estimate:

$$
R_{c}=\frac{1}{2 k a}\left(1+\frac{8}{3 \pi} \frac{\Lambda}{a}\right)
$$

where $\Lambda$ is the phonon mean free path. It follows that if the mean free path increases, so will the constriction resistance.

However, when temperature is further reduced, we obtained a reduction of the measured resistance below $200 \mathrm{~K}$. We provide possible qualitative explanations. First, as explained by Hoogeboom et al.|28], a nanoscale heat source creates an effective phonon modes suppression filter. Phonon modes with mean free path smaller than the heat source will be effectively suppressed. Therefore, to understand the temperature behaviour, the full phonon spectrum needs to be examined as in Wang et al.[134], where they computed the temperature dependence of various modes. 
Linked to this first explanation, a second inspection of Eq. 3.17 provides a possible answer. The ballistic contribution is $R_{b a l l}=\frac{4}{3 \pi a^{2}} \frac{\Lambda}{k}$ is proportional to $\frac{\Lambda}{k}$. In our range of temperatures, both $\Lambda$ and $k$ increase. So, in a general framework, the variation of $R_{\text {ball }}$ depends on the relative changes of $k$ and $\Lambda$. It could be that in different temperature range, different relative changes are received. Furthermore, various phonon modes have various temperature dependence and thermal conductivity contributions. Inspection of the phonon spectrum, mean free path variations and thermal conductivity contribution could provide an answer for the behaviour observed.

Also, the tip and the silicon sample are covered by a native silicon oxide layer of few nanometers. The effect of the native oxide on the resistance is difficult to estimate. It could play a simple interface role but also could act as an extra filter on the phonon propagation, increasing or reducing the phonon modes associated with a given mean free path. We performed an experiment on a silicon sample etched by hydrofluoric acid, measuring its SThM response after various exposure time to ambient air, and therefore increasing native oxide thickness. Results are shown in Fig. 3.13. We obtained a reduction of the SThM response in vacuum which transposes in an increase of the thermal resistance measured. This highlights the effects of the native oxide on the resistance measured. Note that the non-etched response obtained in Fig. 3.13 doesn't follow the trend measured. This can be attributed to etching effect on the surface state. 


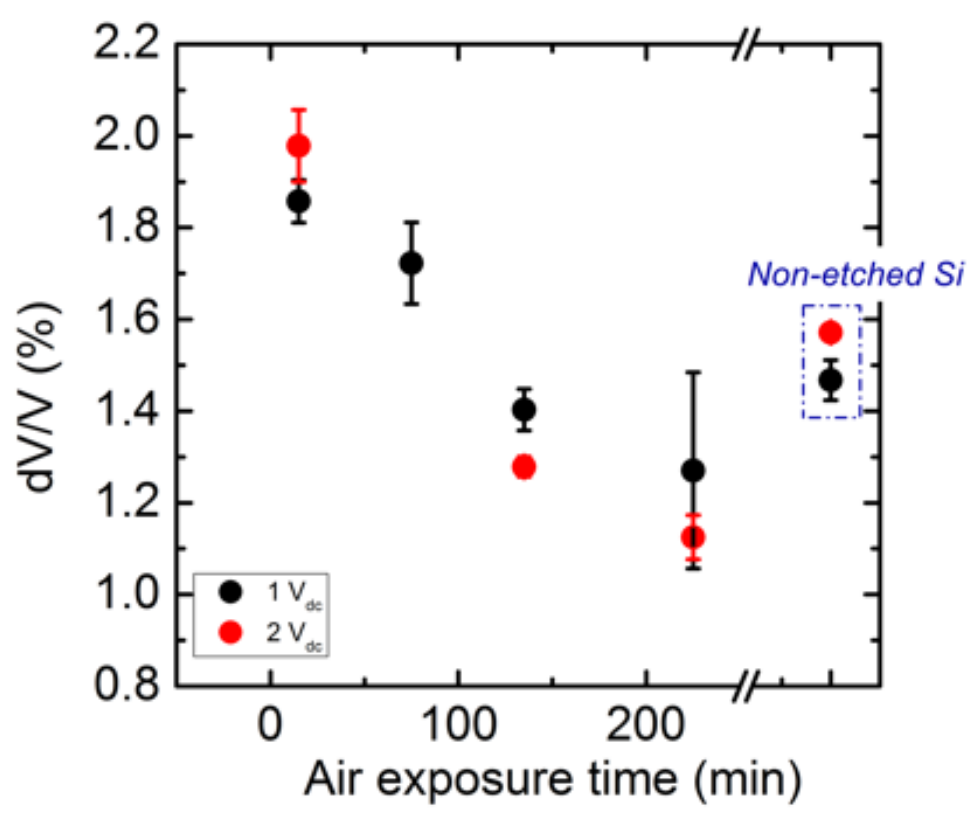

Figure 3.13: SThM response $d V / V$ measured in vacuum as a function of sample air exposition time for two heat temperatures (black and red dots). As time increases, the response diminishes which transposes in an increase of the measured thermal resistance.

Finally, we discuss the contribution of the silicon tip resistance, $R_{t i p}$. For a tip in contact with a sample, almost $90 \%$ of the temperature gradient occurs in the first $100 \mathrm{~nm}$ from the sample within the tip 89. This can be seen on Fig. 3.14 where we used a diffusive model to obtain the temperature distribution in the conical tip in contact with a sample at two different temperatures. The model is presented in the appendices. Therefore, when the tip is in contact with a cooled sample, the portion of the silicon tip that is at cryogenic temperature is small, on the order of $200 \mathrm{~nm}$. In this small part of the tip, thermal transport is dominated by interfaces and the phonon mean free path will be dependent on the probe geometry. Hence, we don't expect to have a strong effect of the cryogenic temperature on the tip thermal resistance. 


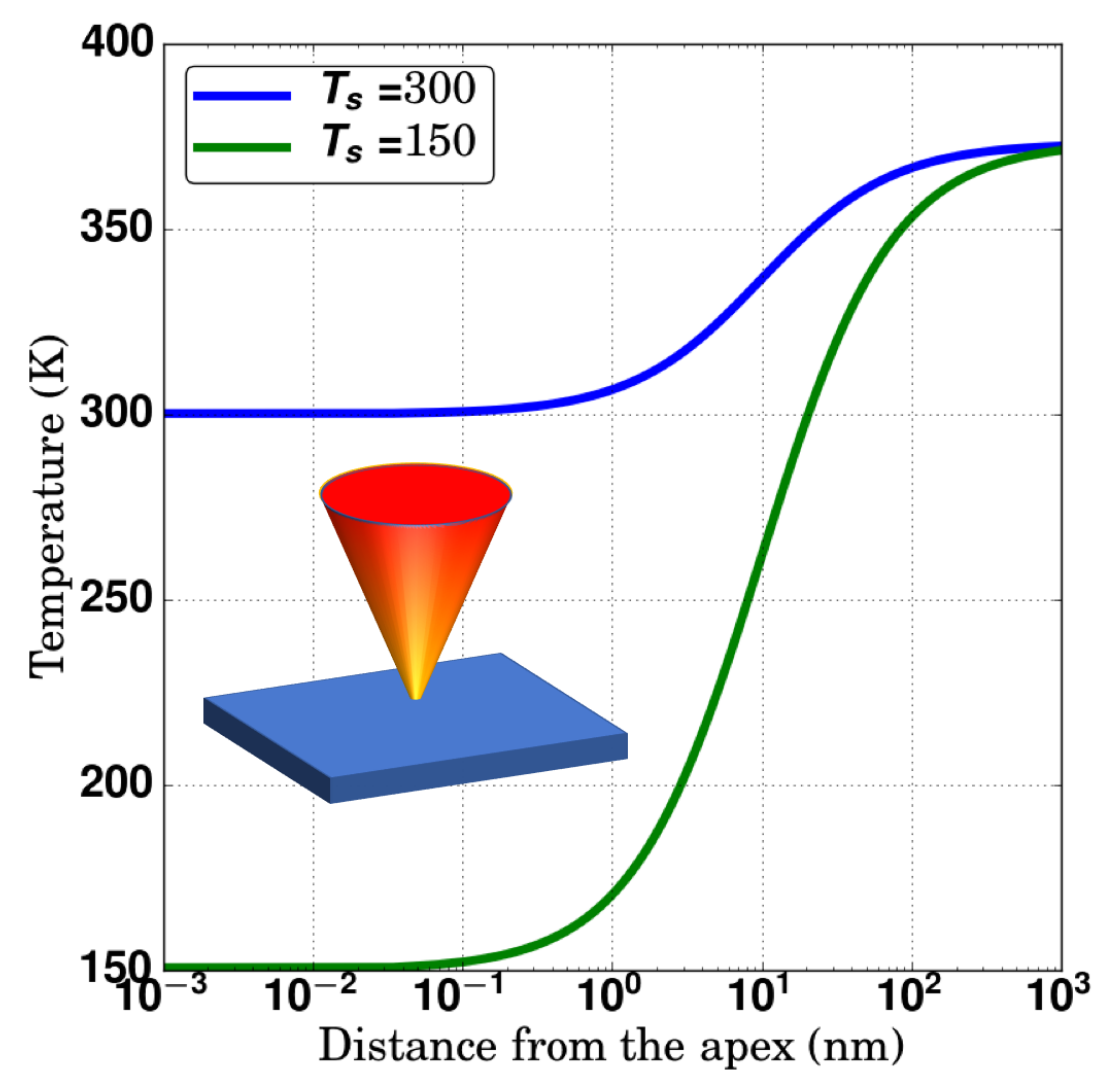

Figure 3.14: Temperature distribution in a conical tip using a diffusive model for two sample temperatures, $300 \mathrm{~K}$ (blue) and $150 \mathrm{~K}$ (green). Most of the temperature drop within the tip occurs within the first $100 \mathrm{~nm}$.

However, to investigate the effect of local sample heating by the probe, we performed measurements using two heater temperatures: 350 and $450 \mathrm{~K}$. It has been shown[50], that samples with high thermal conductivity do not get heated locally by the hot tip. However, for low thermal conductivity samples, some heating is expected. This is observed on the measurements performed on silicon and silicon oxide (see Fig. 3.12). On the silicon, the two heater temperatures produce similar resistance measurements while, on the silicon oxide, the measurements performed with $T_{\text {heater }}=450 \mathrm{~K}$ are lower than measurements with $350 \mathrm{~K}$. We attribute this result to local sample heating from the probe. The silicon high thermal conductivity renders it difficult to heat locally with a nanoscale probe. Thus, the same resistance is produced. The situation is different for the silicon oxide. When the probe is hotter, some volume of the sample gets heated and the thermal con- 
ductivity is no longer the conductivity of the sample heat bath. Higher heater temperature means increase of sample temperature for $\mathrm{SiO}_{2}$ and therefore lower thermal resistance probed.

\subsubsection{Summary}

In this section, we developed and demonstrated cryoSThM measurements. The setup was described and measurements procedure briefly introduced. A dedicated measurement model was developed to translate SThM response $d V / V$ into measured thermal resistance to account for the sample temperature. Then, we demonstrated the cryoSThM measurements of bulk silicon and silicon oxide samples. While measurements of silicon oxide were coherent with the temperature change of thermal conductivity reported in the literature, silicon results were more challenging to interpret. We provided here qualitative explanations and basic mechanisms that can explain the trend received.

We believe that further experiments are needed to fully understand temperature resistance variations of high mean free path materials. The challenge is to understand the various phonon modes contributions to the conductance arising from a heat source smaller than the average phonon mean free path. However, for low thermal conductivity and low mean free paths materials, cryoSThM provides a powerful tool for nanothermal properties investigations. In the next chapters, results using this setup are presented and further demonstrate cryoSThM possibilities.

\subsection{Dynamic SThM: quasi non-contact method for multiparametrical investigation}

In this section, we demonstrate the principle of quasi-non-contact immersion Scanning Thermal Microscopy (iSThM). We show that by combining standard thermal measurements with the principle of Ultrasonic Force Microscopy (UFM) - by def- 
inition a technique which can only operate in the quasi-non-contact regime - we can simultaneously map the thermal and nanomechanical properties of an immersed sample. We show that the application of ultrasound does not significantly affect the thermal response and, furthermore, that via correlation of iSThM and UFM responses during force spectroscopy measurements we can identify optimum conditions for measurements. However influence of ultrasonic displacement and thermal conductance through liquids on measurements has presented interesting challenges to quantitatively understand probe-sample interactions. This requires further complementary modelling.

\subsubsection{Introduction}

Scanning Thermal Microscopy (SThM) is the leading tool for nanoscale mapping of thermal properties, but it is often negatively affected by unstable tip-surface thermal contacts. It would be very tempting to use liquid immersion in SThM to improve both the thermal contact between the probe tip and the sample as well as contact uniformity. The approach would be somewhat similar to one used in ultrasonic imaging where dedicated gel is used to achieve better acoustic coupling or optical microscopy where immersion reduces light reflection, refraction and scattering at the interfaces. This approach is called immersion Scanning Thermal Microscopy (iSThM) and has previously been successfully demonstrated 88 .

Non-contact approaches to SThM are desirable as they provide a method of significantly reducing topographical artefacts which can adversely affect thermal conductivity contrast. However, non solid-solid contact methods are inherently limited for non-flat samples due to problems of accurate probe-sample separation; furthermore mechanical information relating to the sample is not available without solid-solid contact.

Here we propose to combine the uniform thermal contact and massively reduced probe-sample mechanical adhesion 135 of iSThM with the quasi non-contact methodology of Ultrasonic Force Microscopy (UFM). This approach will allow us 
to simultaneously map the thermal and nanomechanical properties of sample and directly correlate contributions to the thermal response arising from intrinsic material properties as well as buried or sample-substrate interfaces. We note that under-liquid UFM feasibility has already been demonstrated 135, 136].

\subsubsection{Methodology}

Ambient and under-liquid (dodecane) SThM measurements were performed using a Bruker Multi-Mode, Nanoscope III with a custom "half-moon" under-liquid SThM probe holder shown in Figure 3.15 and an already described PdRP (Kelvin Nanotechnology).

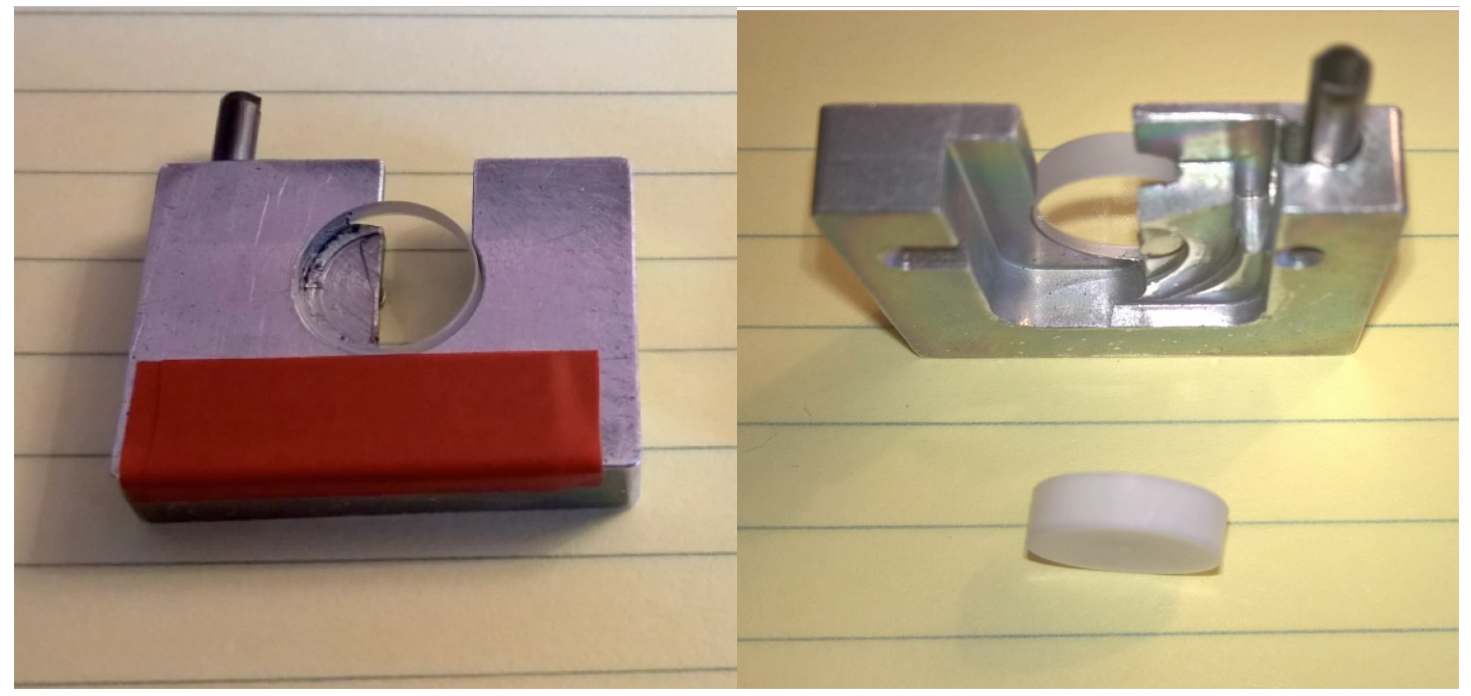

Figure 3.15: Custom under-liquid SThM probe holder. A glass disk is placed above the probe in order to create a meniscus covering the probe and the sample allowing stable refraction of the laser beam.

In both ambient and under-liquid operations, the thermal probe represents part of a balanced Maxwell electric bridge with a $4 \mathrm{~V}_{\mathrm{AC}}$ signal at $91 \mathrm{kHz}$ frequency, provided by a precision function generator (Model 3390, Keithley instruments), used for resistance measurements and a DC offset providing probe Joule self-heating [50]. The probe's electrical resistance was recorded as a function of applied voltage and temperature, using the method described elsewhere 75,88 . For UFM, samples were mounted on a piezoceramic transducer (Physik Instrumente) using a thin layer of water-soluble high temperature wax resulting in good coupling of longi- 
tudinal and shear ultrasonic vibrations at frequencies up to several tens of MHz. The sample-piezotransducer system was then bonded inside a Teflon bowl (Figure 3.16) which in turn was mounted on a metallic disk.

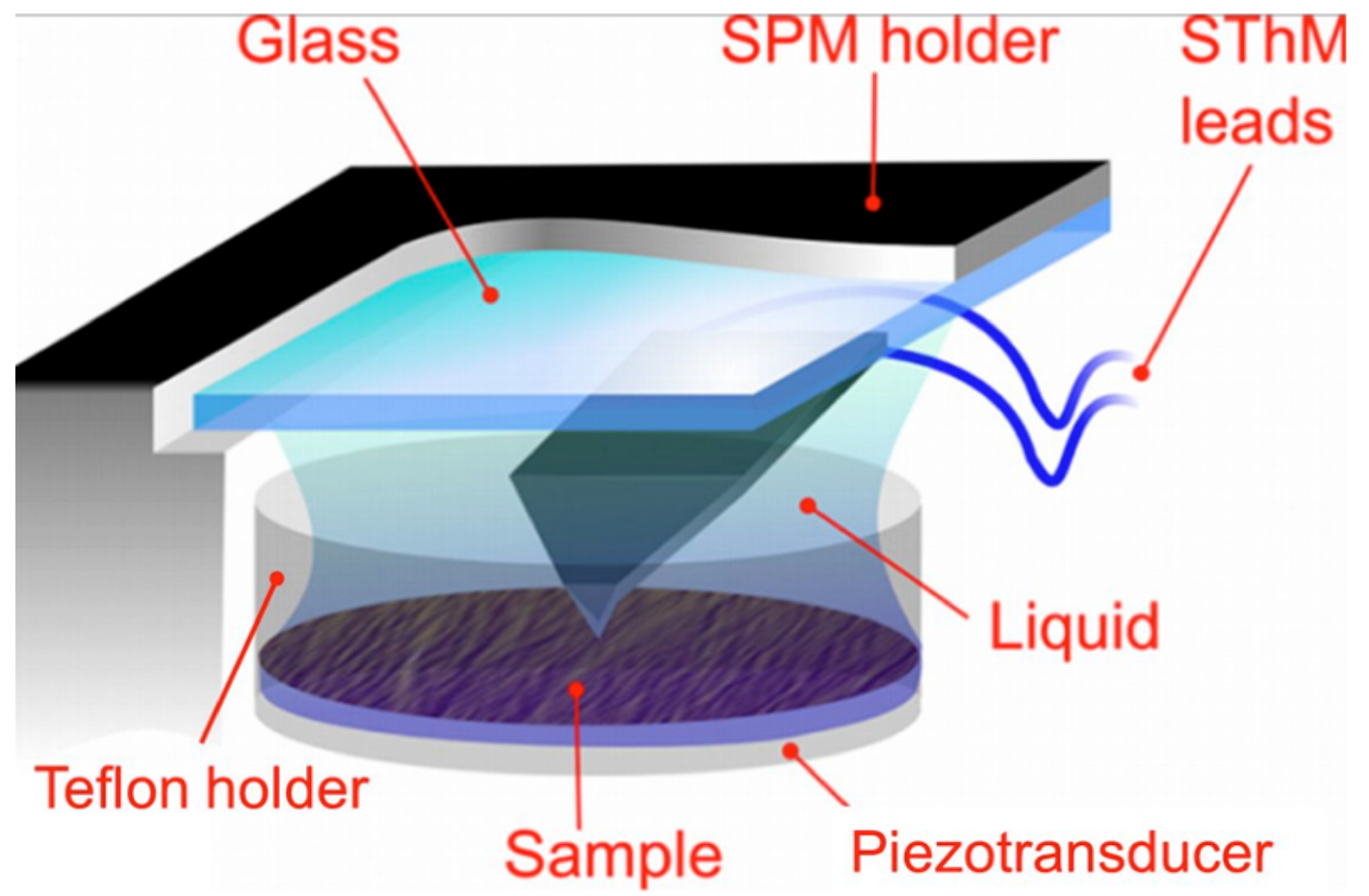

Figure 3.16: Schematic representation of the liquid Teflon holder containing the piezotransducer and the sample.

Transducer mounted samples were oscillated at typically $4 \mathrm{MHz}$, well above any cantilever resonances, with a peak to peak amplitude of $1,2,3$ or $4 \mathrm{~V}$ resulting in vertical displacements $a$ of $c a .0 .5,1,1.5$ and $2 \mathrm{~nm}$, respectively, as determined by laser Doppler vibrometer (Polytec Inc). These result data are consistent with previously reported values of displacement 36,137$]$. To enable detection of the $4 \mathrm{MHz}$ oscillation, the signal amplitude was modulated with a gated saw-tooth shaped waveform of $1.7 \mathrm{kHz}$, the cantilever deflection at this modulation frequency corresponds to the nonlinear UFM response and was subsequently detected by a lock-in amplifier (Stanford Research Systems, SRS-830) as well documented in literature 4, 137] . In addition to image channels (topography, SThM and UFM), we collected normal force spectroscopy (approach-retract curves 138, 139]) enabling quantitative comparison of the nonlinear and thermal responses and identification of the quasi-non-contact regime. 


\subsubsection{Results}

Figure 3.17 shows the simultaneous scanning of topography, UFM and SThM for an exfoliated multi-layer graphene flake on $\mathrm{SiO}_{2}$ substrate in ambient conditions and immersed in dodecane solution. Furthermore, for comparison, the imaging for both environments without UFM switched on are also given.
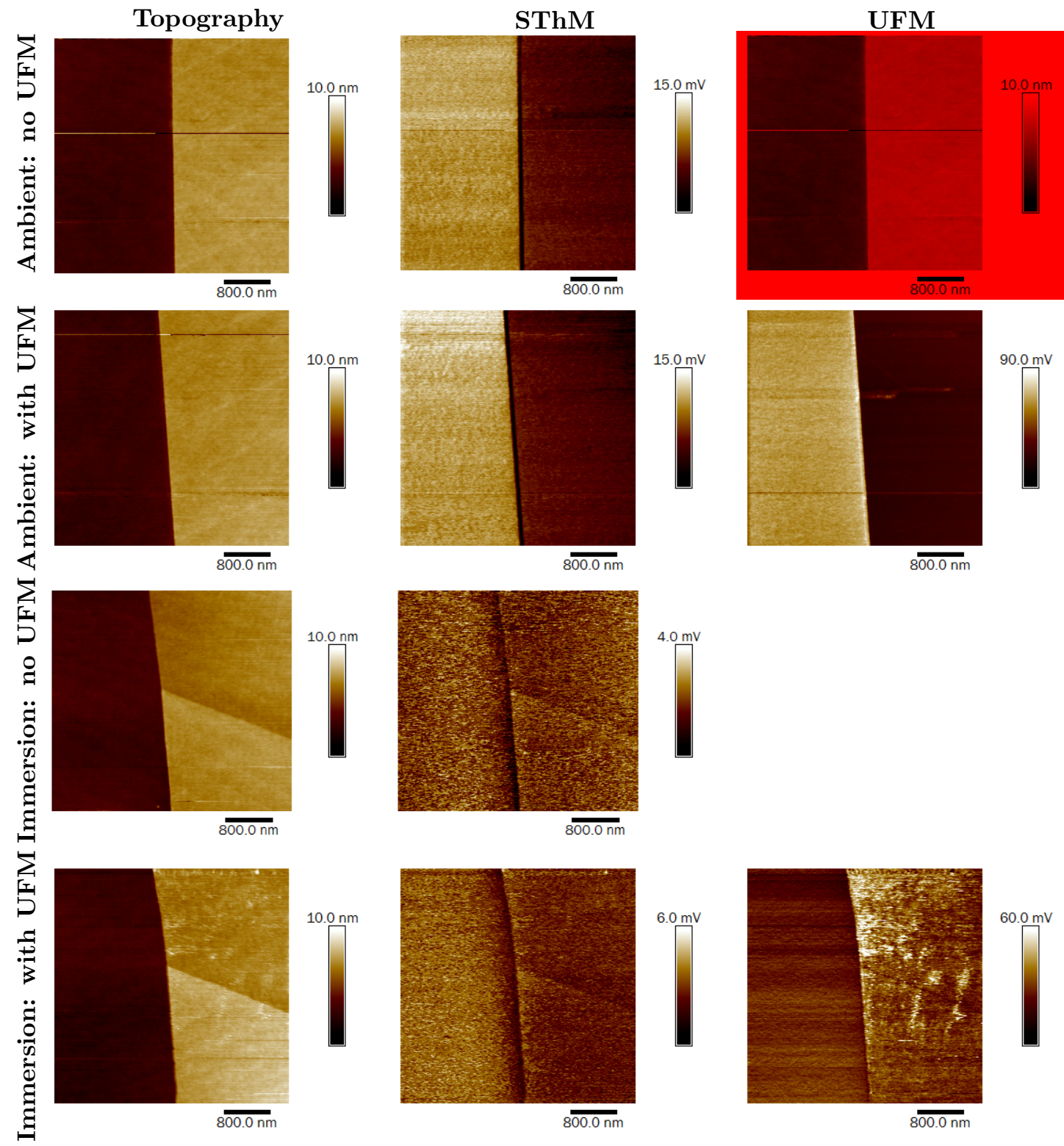

Figure 3.17: Topographical, SThM (thermal) and UFM (nanomechanical) responses with UFM off and on, in ambient and dodecane liquid environments. All channels are captured simultaneously using a KNT probe operating in active mode. The sample is an exfoliated multi-layer graphene flake on a $\mathrm{SiO}_{2}$ substrate.

We clearly see the feasibility of both iSThM and the simultaneous monitoring of both thermal and nonlinear nanomechanical responses in ambient and immersed 
conditions. We elucidate the nature of the probe-sample interaction, whilst immersed, via approach-retract spectroscopy of all three channels, shown in Figure 3.18. Here we show the responses obtained on $\mathrm{SiO}_{2}$ however graphene results are comparable.

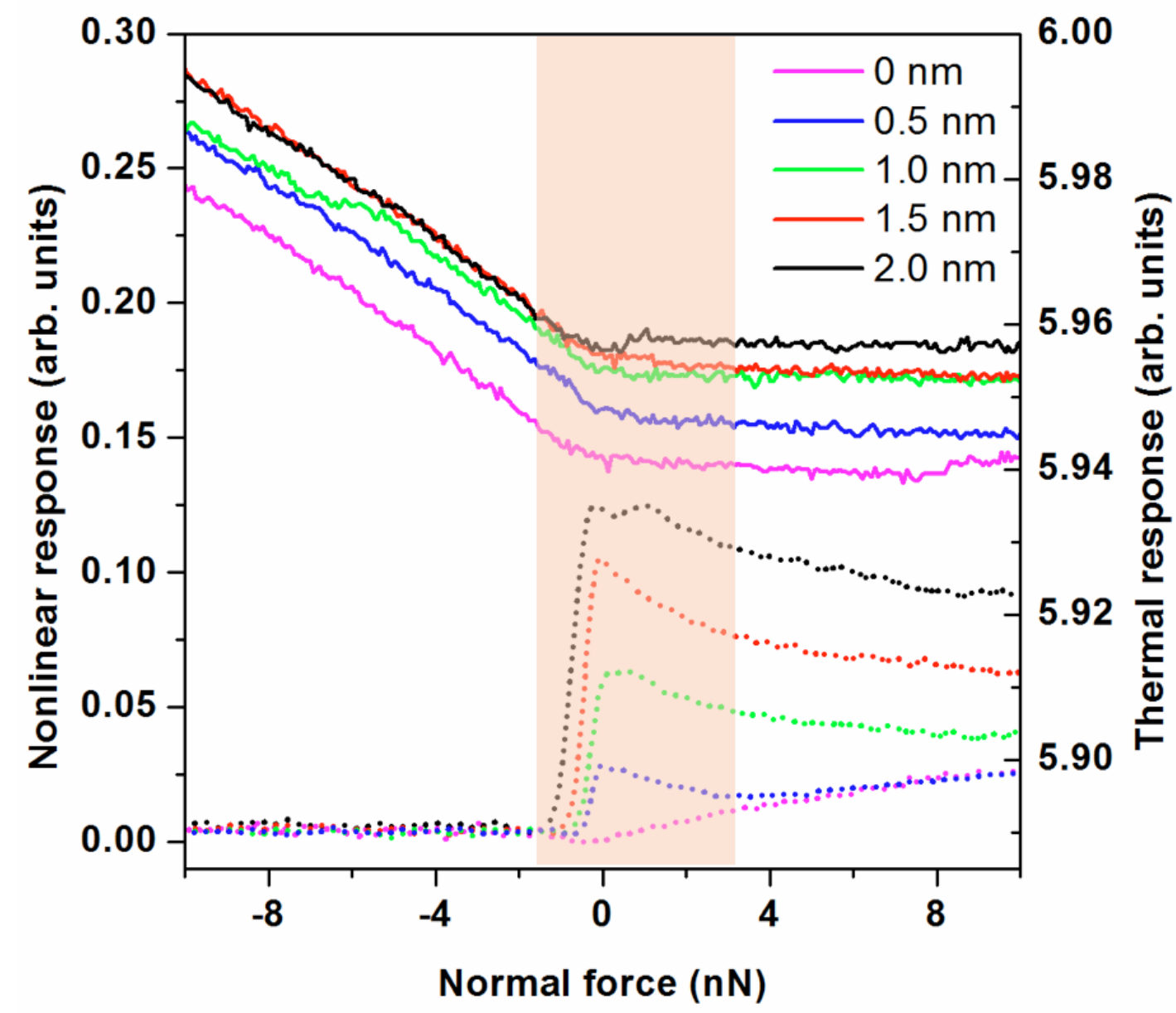

Figure 3.18: SThM (plain lines) and UFM spectroscopic (dotted lines) responses as a function of normal force during probe approach to $\mathrm{SiO}_{2}$ substrate in dodecane immersion for various oscillation amplitude. The quasi non-contact regime is signified by the orange shaded region.

Forces were calculated from the linear region of the approach-retract curve which demonstrated no discernible snap-in and negligible $(<5 \mathrm{nN})$ adhesion forces during retraction; the measured cantilever z-position is used to correlate the different channel responses. During SThM approach, we observe a smooth transition between non-contact and contact regions (here $0 \mathrm{nN}$ corresponds to the sample surface position) due to lack of snap in and uniform thermal conductance to the liquid. Significantly, we can identify peak nonlinear response in the UFM channel 
close to the onset of solid-solid probe-sample contact, which reduces at higher normal forces as expected. Unlike ambient conditions, UFM spectroscopy response does not return to zero at higher normal forces due to acoustic pressure exerted on the cantilever in viscous dodecane[136]. By definition, UFM nonlinear response is only observed in the quasi-non-contact regime, we can therefore accurately identify the normal forces necessary to achieve the equivalent quasi-non-contact iSThM, indicated by the orange shaded region in Figure 3.18 .

In UFM spectroscopy measurements, the sample is vibrated in the oscillating (harmonic) way at high frequency (HF) well above cantilever free and contact resonances. Due to the extreme dynamical rigidity of the cantilever the vibration of the sample is not transferred to the cantilever and therefore one can safely assume that the tip-surface distance is also oscillated at the same ultrasonic frequency $\mathrm{f}$ and the amplitude a. Due to the highly nonlinear dependence of the interaction force on the tip-surface distance 36,37 such oscillation is "rectified" producing an additional "ultrasonic" force that can be defined as a function $F_{m}\left(h_{1}, a\right)$ dependent on the initial indentation $h_{1}$, the ultrasonic amplitude $a$ and original force-vsdistance dependence in the absence of the ultrasonic vibration $F(h)$. The new force $F_{m}$ can be calculated as follows:

$$
F_{m}\left(h_{1}, a\right)=\frac{1}{T} \int_{T} F\left(h_{1}-a \cos (2 \pi f t)\right) d t
$$

where $F(h)$ is the force dependence on the indentation depth without ultrasonic vibration and the integral is taken over a period $T=1 / f$. The initial cantilever deflection $z_{0}$ is defined by set force $F_{0}=k_{c} z_{0}$ where $k_{c}$ is the cantilever spring constant, however the additional ultrasonic force leads to an additional cantilever deflection $z_{a}$ such that new equilibrium position of the cantilever $z=z_{0}+z_{a}$. From Figure 3.18 , this change in equilibrium position may have a small offset effect on SThM without altering either its relative response (non-contact to contact) or shape, however it is more likely that this offset arises due to the acoustic pressure exerted on the cantilever by the ultrasonic oscillations of the sample. 
Further quantification using a combined nanomechanical indentation model (comprising three components describing the classical Hertzian contact force between a sphere and flat plane $\left(F_{\text {Hertz }}\right)[140]$, Van der Waals adhesive 141] between tip and sample $\left(F_{D M T}\right)$ (the Derjaguin-Muller-Toporov model) and short-range hydration forces in water, $\left(F_{H y d}\right)[142$ and nanoscale thermal contact models will be explored.

\subsubsection{Summary}

- We have demonstrated quasi non-contact SThM in ambient and liquid immersion (iSThM) environments via the principle of UFM allowing significant reduction of the shear (friction) forces without affecting the SThM signal.

- We show that a small offset in thermal response is experienced during combined scanning which we believe is due to acoustic pressure acting on the cantilever.

- By monitoring the peak-nonlinear response we can accurately identify the optimum applied normal force between probe and sample to achieve quasinon-contact scanning.

- Working in this optimal regime allows simultaneous monitoring of both thermal and nanomechanical properties of immersed samples.

- Further investigation of the true nature of the nanoscale thermal contact during ultrasonic oscillation is required and will be performed using a combination of analytical and finite element modelling. 


\section{Quantitative thermal transport}

\section{measurements in nanostructures}

Based on the experimental and analytical framework developed in the previous chapter, we develop here the requirements needed for quantitative comparison and estimation of thermal properties. Using an analytical model for the thermal spreading resistance in the sample, quantities such as thermal conductivity and interface thermal resistance can be deduced from SThM measurements. We apply similar models to relatively simple samples, oxide layers on silicon substrates to demonstrate the principles underlying these models. Finally, metal covered block copolymers thin films are mapped and their thermal resistances measured to extract effective thermal conductivities.

\subsection{Principles and applications of layer analytical model}

The previous chapter demonstrated how to understand an SThM measurement in terms of thermal resistance. Different probes need different models to extract the thermal resistance out of the measured signal. Once a proper model is developed, the interpretation starts. We highlight here again that the measurement output is a thermal resistance. This resistance has to be understood in light of the system particularities.

For example, when measuring bulk samples, the thermal resistance measured depends on three parameters (in a simplified case): the resistance from the sensor 
to the tip apex $R_{\text {tip }}$, the interface resistance between the apex and the sample $R_{\text {int }}$ and the spreading resistance $R_{s p r}$. Let's assume the goal of the measurement is the thermal conductivity of the given sample. This parameter is mostly hidden within the spreading resistance which in the case of bulk sample is expressed as $R_{s p r}=\frac{1}{4 k a}$ with $a$ the contact radius and $k$ the thermal conductivity. Even if we know exactly the contact radius and $R_{\text {int }}$ which is a difficult task on its own, $R_{\text {int }}$ remains coupled with $R_{\text {spr }}$. $R_{\text {int }}$ can be modelled and measured with other techniques but it is never certain that these models and measurements represent the same physics as $R_{\text {int }}$, especially in the case of a nanoscale probe. In summary, extracting material properties from a single measurement is a very difficult task.

Figure 4.1 shows the SThM response $d V / V$ as a function of thermal conductivity of the bulk material. As we observe while measurements are generally reproducible, the two quantities can hardly be correlated. Whether this arises from nanoscale phenomena or different sample surface states is debatable. However, it shows that comparison between different SThM experiments can be difficult, even when using the same setup.

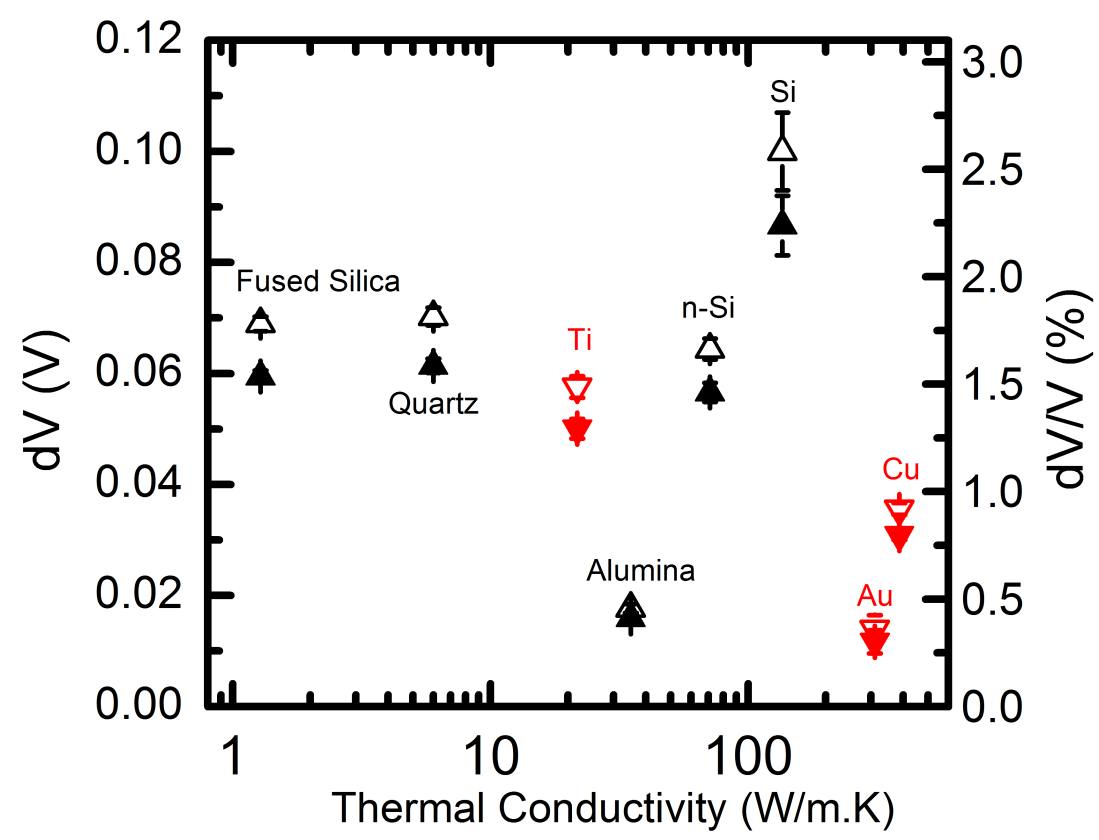

Figure 4.1: SThM response $d V / V$ as a function of bulk sample thermal conductivity for metallic (red) and non-metallic (black) samples measured in ambient air conditions. Even within one type of sample, it is difficult to extract a link between thermal conductivity and SThM response. 
How could then SThM measurement become quantitative and produce material properties quantitative insights? One approach, presented in the next chapter, uses a new technique able to output material properties such as thermal conductivity and interface conductance from a single sample using varied thickness of a sample. This technique can also be used in a simpler form that we demonstrate here.

The background assumption is the following. From two thermal resistance measurements $R_{X_{1}}$ and $R_{X_{2}}$, the variation $\Delta R$ depends only on the spreading resistance variation:

$$
\Delta R=R_{X_{2}}-R_{X_{1}}=R_{t i p}+R_{i n t}+R_{s p r_{2}}-R_{t i p}+R_{i n t}+R_{s p r_{1}}=R_{s p r_{2}}-R_{s p r_{1}} .
$$

For this assumption to be valid, both $R_{t i p}$ and $R_{\text {int }}$ need to be the same for the two samples. If $R_{t i p}$ is likely to stay the similar between various experiments, $R_{\text {int }}$ will depend on many parameters such as surface roughness and chemistry, or material properties. Thus, comparison can only be valid if $R_{\text {int }}$ can safely be assumed the same. This is typically realised when the same materials are creating the tip-sample interface but also when these materials where prepared using the same preparation procedure.

Once this assumption is valid, the measurement difference $\Delta R$ can be understood in terms of material properties. Spreading resistance as we discussed earlier, can be modelled analytically for simple systems whereas more geometrically complex samples can be modelled with finite elements programs as we show in the next chapter. We then proceed to demonstrate how to obtain quantitative estimates of materials properties using these requirements. Two types of samples are used: silicon oxide and aluminium oxide layers. At the end of this chapter, similar models are used for modelling thermal transport in nanostructures.

To present the underlying method used below, we first demonstrate it on simple systems. The first samples "set" is several silicon oxide steps thermally grown on a silicon substrate (see Fig. 4.2 a). These steps are selectively etched to create like a staircase with each step having a different thickness. As these steps were grown 
on the same substrate, they don't really form a sample set composed of different samples. However this sample provide a very useful standard system as it doesn't require extensive experimental manipulation. The second set is made of several aluminium oxide layers grown on a silicon substrate using atomic layer deposition (ALD). In this case, different Si substrates were used for the different thicknesses. The oxide steps sample was fabricated by partners at Glasgow University and Kelvin Nanotechnology while the ALD was performed by Picosun.

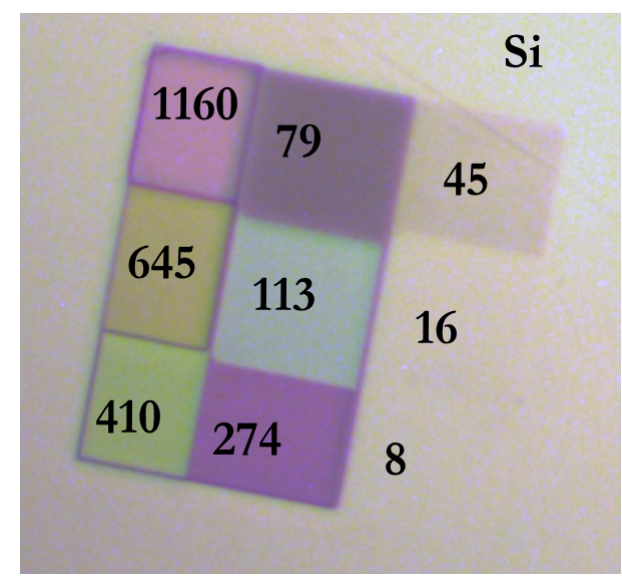

Figure 4.2: Optical microscope image of the silicon oxide steps. Each step is around $10 \times 10 \mu \mathrm{m}^{2}$ and its thickness indicated in $\mathrm{nm}$.

To model such systems of layer on substrate, we introduced the spreading resistance models in the background review chapter 2. We use the analytical expression derived by Yovanovich and Muzychka 103, 105 for an isotropic layer on a isotropic substrate. When the probe contacts the sample surface, heat is transferred to the sample and this thermal resistance from the probe to the sample is measured through the probe's electrical resistance. We can split this resistance into two main components: the interface between the sensor and the sample and the thermal spreading resistance:

$$
R_{X}=R_{\text {int }}+R_{\text {spr }}
$$

Further distinction can be made for the former for example by distinguishing heat resistances of the tip itself and the tip-sample interface. The latter can also 
be split into several components such as the water meniscus conductance 83 and solid-solid contact interface. Here we consider an effective interface resistance including all components. As often in SThM measurements, we consider the tip in contact with the sample surface as a disk of radius $a$. Water meniscus and multi-asperity contact are included in this effective radius which might not then represent the exact physical dimension of the probe-sample contact.

The thermal spreading resistance depends on the structure and materials composing the sample. In the simple case of a bulk isotropic material, it is given by:

$$
R_{s p r}=\frac{1}{4 k a}
$$

with $k$ the thermal conductivity of the material and $a$ the effective contact radius between the probe and the sample.

In the present case, we are dealing with a single layer on a substrate. Therefore, we need to develop a model taking into account the heat spreading in the layer resting on the substrate as well as the interface resistance between the layer and the substrate. Muzychka et al. 105, 107 derived a model using an effective thickness defined by the interface resistance $\left(t_{\text {eff }}=t+r_{\text {int }} k_{\text {layer }}\right)$. Note that this model can be extended to an orthotropic system 113 but here we use a simplified form.

$$
R_{\text {spr }}(t)=\frac{1}{\pi k_{\text {layer }} a} \int_{0}^{\infty}\left[\frac{1+K \exp \left(\frac{-2 \xi t_{\text {eff }}}{a}\right)}{1-K \exp \left(\frac{-2 \xi t_{\text {eff }}}{a}\right)}\right] J_{1}(\xi) \sin (\xi) \frac{d \xi}{\xi^{2}}
$$

where $J_{1}$ is the first Bessel function of the first kind and where $K$ is defined as $K=\frac{1-k_{\text {sub }} / k_{\text {layer }}}{1+k_{\text {sub }} / k_{\text {layer }}}$. If the layer is thick enough, the spreading resistance returns to $\mathrm{Eq} 4.3$.

Thus, assuming that $R_{\text {int }}$ is independent on the thickness, Eq. 4.2 becomes:

$$
R_{X}(t)=R_{\text {int }}+R_{\text {spr }}(t)
$$

with all changes in the measured thermal resistance arising from the thickness 
variation. Eq. 4.4 can then be used to model those changes and extract materials properties.

\subsubsection{Silicon oxide steps}

We measured the SThM response $d V / V$ of each step using the setup described in the previous chapter 3 and converted this response in thermal resistance using the model derived in the same chapter. We can therefore fit the analytical expression from Eq. 4.2 to our data. There are four unknowns: the interface resistance $R_{\text {int }}$, the contact radius $a$, the thermal conductivity of the layer $k$ and the interface thermal resistance between the layer and the substrate $r_{\text {int }}$. Figure 4.3 shows the experimental data and the fitted curve. To fit the model to the experimental data, we applied some reasonable boundaries to the fitting parameters. For example, we would not expect a contact radius smaller than $20 \mathrm{~nm}$ and bigger than $200 \mathrm{~nm}$, or a thermal conductivity bigger than $10 \mathrm{Wm}^{-1} \mathrm{~K}^{-1}$.

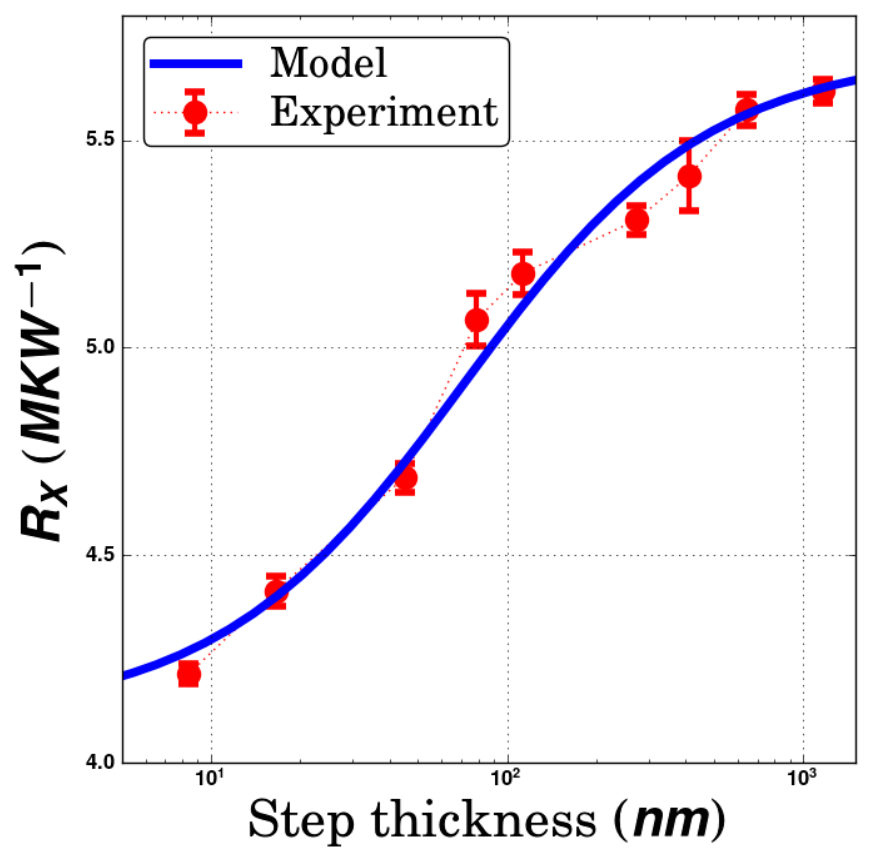

Figure 4.3: Oxide steps thermal resistance measured and the fitted curve using the spreading resistance model with the following parameters: $R_{\text {int }}=2.6 \times 10^{6}$ $\mathrm{KW}^{-1}, a=65.5 \mathrm{~nm}, k=1.0 \mathrm{Wm}^{-1} \mathrm{~K}^{-1}$ and $r_{i n t}=4.91 \times 10^{-8} \mathrm{~m}^{2} \mathrm{KW}^{-1}$.

We obtained a realistic contact radius of $a=65.5 \mathrm{~nm}$ as well as for the ther- 
mal conductivity of the silicon oxide layers $k=1.0 \mathrm{Wm}^{-1} \mathrm{~K}^{-1}$ being in a good agreement with optical technique measurements of similar sample[143. The value of interface thermal resistance obtained $\left(r_{i n t}=4.91 \times 10^{-8} \mathrm{~m}^{2} \mathrm{KW}^{-1}\right)$ is around one order of magnitude higher than that found by Chien et al. 144 but similar to that found by Zhu et al. 145.

\subsubsection{ALD aluminium oxide layers}

A similar experiment was then performed on ALD grown aluminium oxide samples. The sample set consists of 9 samples with thicknesses increasing from $2 \mathrm{~nm}$ to 500 $\mathrm{nm}$. Using the same procedure, we fitted the results with the spreading resistance model. Figure 4.4 shows the results. We received a higher contact radius $a=130$ $\mathrm{nm}$ which is still plausible as a different probe was used and as this effective radius includes all heat transfer effects from the probe to the sample. The thermal conductivity obtained is $k=1.81 \mathrm{Wm}^{-1} \mathrm{~K}^{-1}$ which is close to the value obtained by Al Mohtar et al. 143 on similar samples. Again we note a high interface thermal resistance $r_{i n t}=4.95 \times 10^{-8} \mathrm{~m}^{2} \mathrm{KW}^{-1}$. This high value could arise from a high inhomogeneous interface from the ALD deposition. 


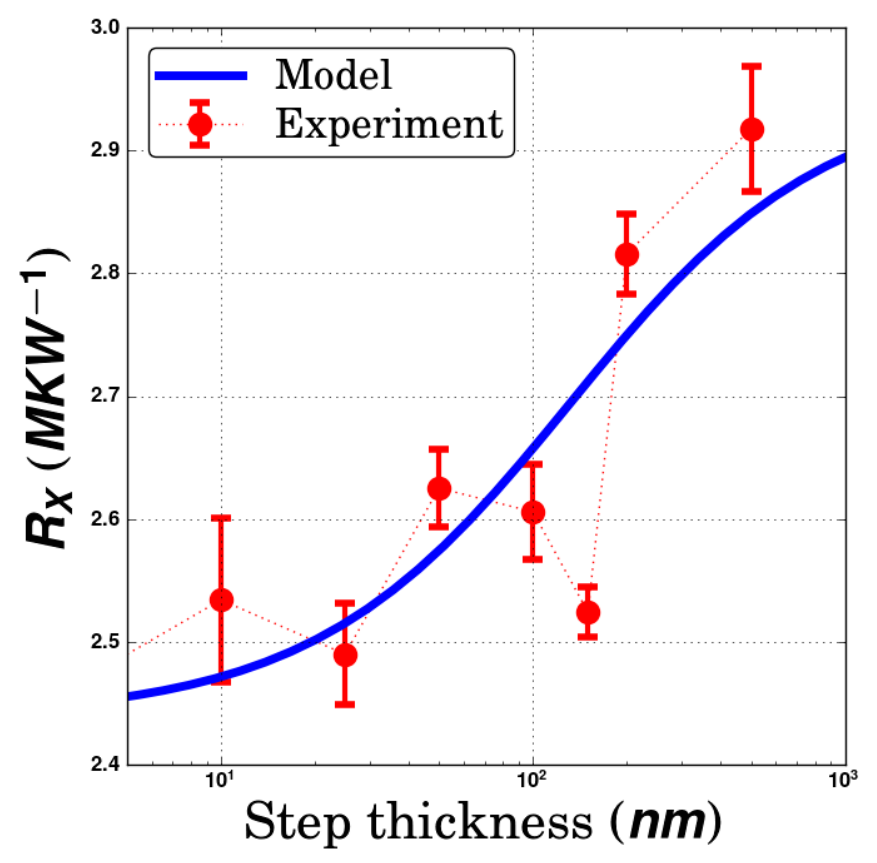

Figure 4.4: Aluminium oxide layers thermal resistance measured and the fitted curve using the spreading resistance model with the following parameters: $R_{\text {int }}=$ $2.1 \times 10^{6} \mathrm{KW}^{-1}, a=130 \mathrm{~nm}, k=1.81 \mathrm{Wm}^{-1} \mathrm{~K}^{-1}$ and $4.95 \times 10^{-8} \mathrm{~m}^{2} \mathrm{KW}^{-1}$.

\subsection{Thermal transport in metal covered block copoly-}

\section{mers}

In this section, we report SThM measurement performed in vacuum $\left(\sim 10^{-6}\right.$ Torr $)$ with the doped silicon probe. First we present results on chromium covered block copolymer layers. These layers are mostly used for nanofabrication process. Therefore knowing their thermal properties can be essential for procedure improvement. These samples were fabricated at the Catalan Institute of Nanoscience and Nanotechnology (ICN2) and the work presented here was performed in partnership with Dr. Alexandros El Sachat and Dr. Francesc Alzina.

The block copolymer (BCP) consisting of mixed polyethylene oxide (PEO) and polystyrene (PS) presents interesting self-arrangement. Depending on the fabrication process, either pillars or lines morphologies are observed. The fabrication is as follows (see Fig, 4.5 a). First, a solution is spin coated on a silicon substrate. Then upon annealing microphase separation occurs in PEO pillars embedded in a 
PS matrix. If during the annealing step, a mixed chloroform and toluene atmosphere is introduced, the microseparation provokes PEO lines formation on a PS layer. Then, BCP layers are covered by a chromium layer by thermal evaporation. The chromium thickness was varied from 0 to $5 \mathrm{~nm}$. SEM images of the different morphologies are presented in Fig. $4.5 \mathrm{~b}-\mathrm{f}$.
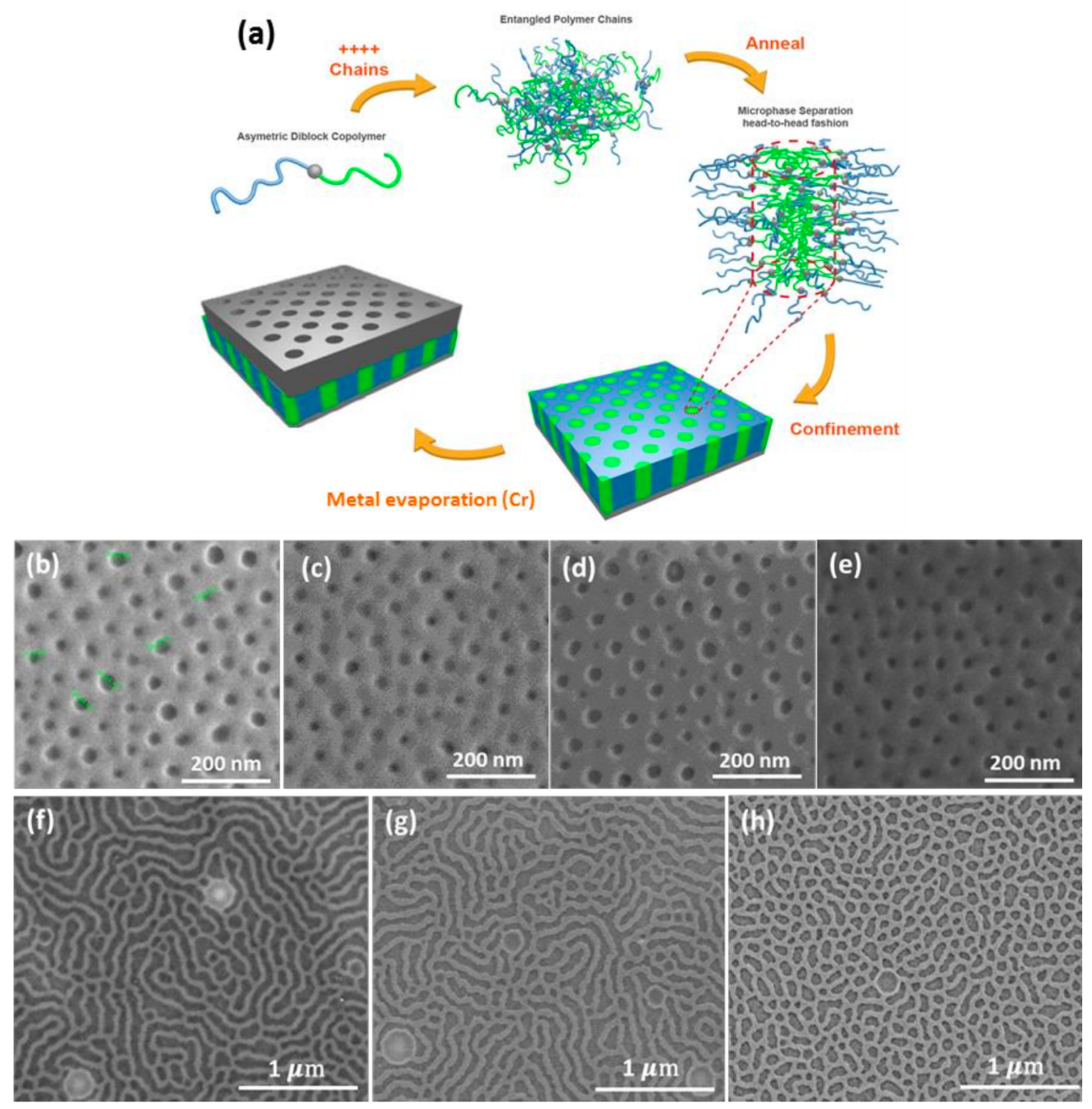

Figure 4.5: (a) Schematic illustration of the fabrication process. SEM images of (b) the fabricated microphase separated BCP film on Si substrate and the BCP templates with nominal Cr coatings of (c) $1 \mathrm{~nm}$, (d) $2 \mathrm{~nm}$ and (e) $5 \mathrm{~nm}$. The darker regions are the PEO domains that were degraded, and the lighter region the PS matrix. SEM images of (f) the fabricated BCP template after the solvent annealing showing the modified morphology and the BCP templates with (g) 2 $\mathrm{nm}$ and (h) $5 \mathrm{~nm}$ Cr layers

First we study individual regions of the BCP films to understand how the interfaces between the two blocks after the reorganization of their chains in the phase separated structure affect the heat dissipation in their volume. In Fig. 4.6 we 
present topography and thermal resistance images of the BCP templates without Cr layer obtained by simultaneous mapping the local variations in height (Figs. 4.6 a,c) and tip-sample thermal resistance (Figs. 4.6b,d). The high-resolution thermal image of the BCP template with the cylinder morphology shows thermal contrast between the PS matrix and the PEO cylindrical domains. The PEO phase of the BCP appears darker than the PS matrix in the thermal image (figure $4.6 \mathrm{~b}$ ), indicating a reduced tip-sample heat flux when the tip is in contact with the PS matrix. However, in the $\mathrm{BCP}$ template with the lamellae morphology the thermal contrast is reversed (see Figs. 4.6).

\section{Topography}
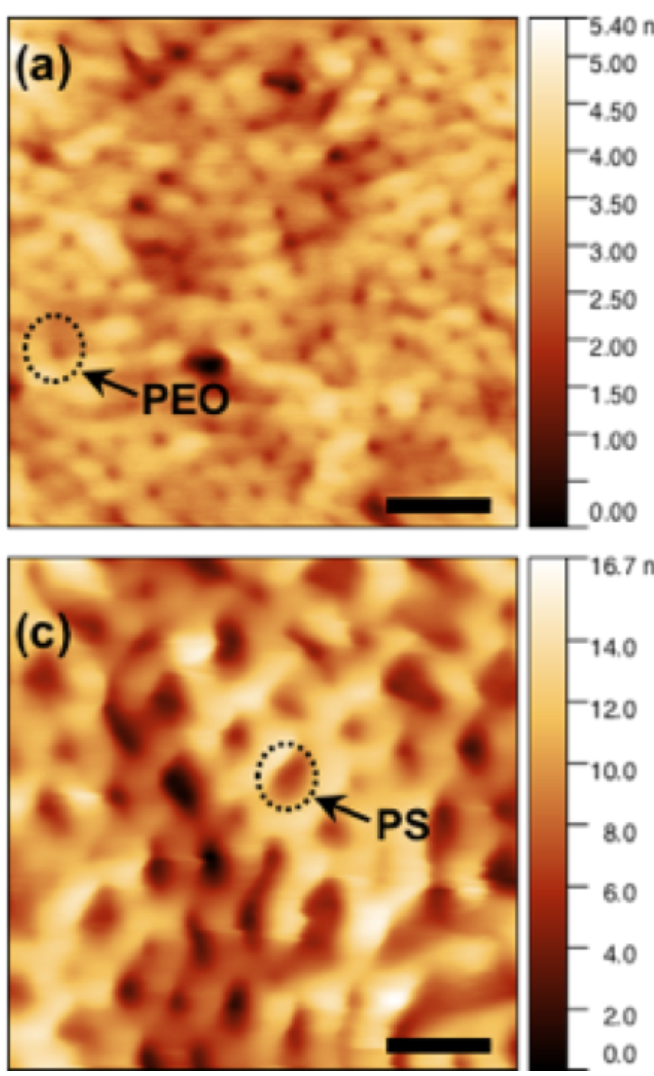

\section{SThM}
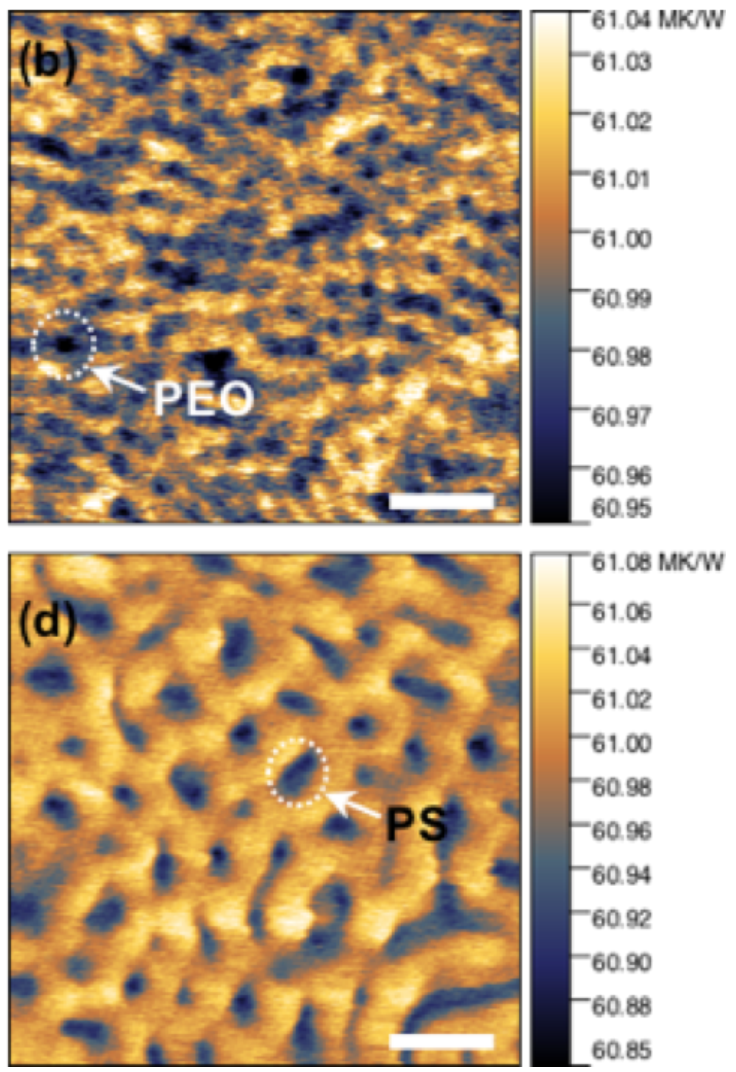

Figure 4.6: (a),(c) Topography and (b),(d) thermal resistance images of the nominal BCP templates. Scale bars are $100 \mathrm{~nm}$. PS and PEO regions are indcated by arrows.

Since the two polymers have similar intrinsic thermal conductivities, the origin of the contrast could be related to a topography artefact. Nevertheless an alternative explanation is possible taking into account that heat spreading to the 
substrate is present and, consequently, affects the tip-sample heat transfer. For a given sample, either the pristine $\mathrm{BCP}$ template or a $\mathrm{BCP}$ with a $\mathrm{Cr}$ layer, if there are no spatial variations of the thermal conductivity and of the Cr layer thickness, the only parameter that can change the heat spreading to the substrate is the thickness of the BCP. As the samples show a marked surface topography with the PEO cylinders thinner that the PS matrix, this BCP thickness modulation results to a related thermal conductance change and in the contrast in the thermal images. Therefore, the thermal contrast is not related to variations of the thermal conductivity between constituent blocks, but to the relative thickness of the different domains and, therefore, to the distance of the heater to the substrate. The same trend has been observed in the BCP templates with different Cr layers as is shown in Fig.4.7. 


\section{Topography}
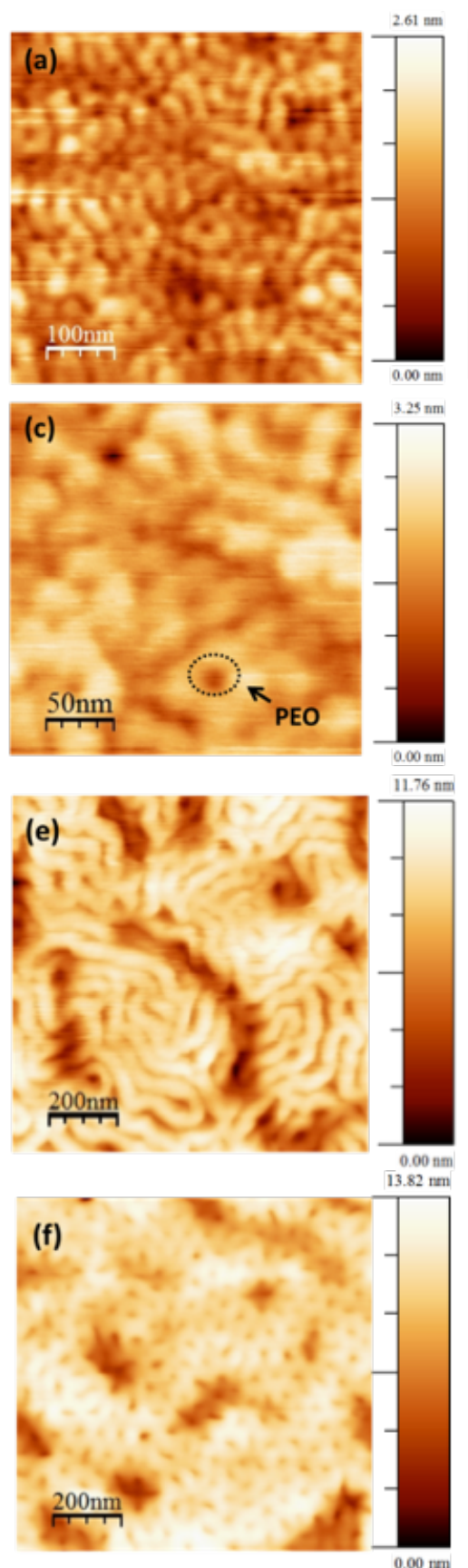

SThM
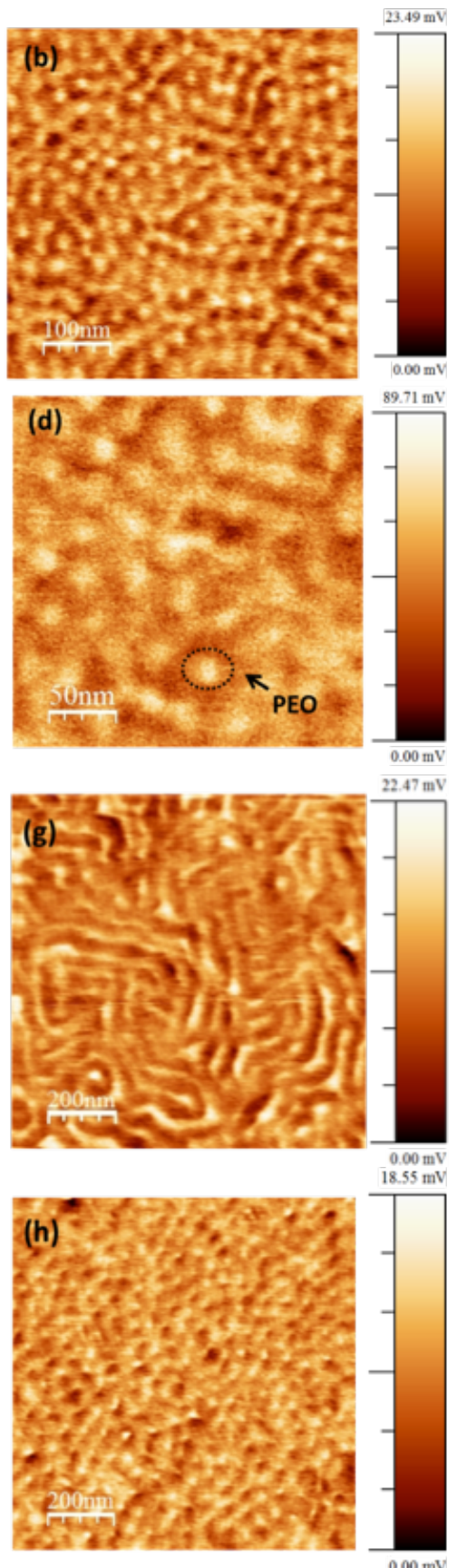

Figure 4.7: (a),(c) Topography and (b),(d) thermal images of the BCP template with $1 \mathrm{~nm}$ Cr layer. Topography and thermal images of the modified BCP templates with 2 (e),(g) and (f),(h) $5 \mathrm{~nm}$ Cr layers, respectively.

Next we apply a model in order to gain more quantitative information regarding the heat dissipation in the BCP films. Particularly, when the probe is in contact with the sample, the heat generated in the SThM heater can only transfer to two heat sinks: the probe cantilever to the microscope and the sample. By monitoring 
the change in probe excess temperature in and out contact, we measure the total thermal resistance between the tip and the sample as explained in chapter 3

Considering the very low thermal conductivity of the BCP films, here we assume the thermal transport as entirely diffusive. This is supported by the high probability of phonon scattering whithin polymer chains. Thus the phonon MFP is considered very small for this system. Therefore, similar analytical expressions as used in previous sections for the estimation of the $R_{s p r}$ can be used. However, in this case, the system is made of two layers on a substrate. A model for multilayer contacts was also derived by Muzychka et al.|104|. The solution was derived for an isothermal heat flux on a tube of radius $b$ which accounts for boundary effects. In our case these effects can be neglected and taking $b$ arbitrarily large corresponds to the half-space solution. The mathematical treatment is heavier than the single layer case. The spreading resistance of a double coated surface is given by the following equation:

$$
R_{\text {spr }}^{2-\text { layers }}=\frac{\Psi}{4 k_{3} a}
$$

with the spreading resistance parameter given by

$$
\Psi=\frac{16}{\pi \epsilon} \sum_{n=1}^{\infty} \frac{\delta_{n} \epsilon}{2} \frac{J_{1}\left(\delta_{n} \epsilon\right)}{\delta_{n}^{3} J_{0}^{2}\left(\delta_{n}\right)} \frac{\phi^{+}}{\phi^{-}} K_{21} K_{32}
$$

where $\phi^{ \pm}=\left(1+K_{21}\right)\left(1+K_{32}\right) \pm\left(1-K_{21}\right)\left(1+K_{32}\right) e^{-2 \delta_{n} \epsilon \tau_{1}}+\left(1-K_{21}\right)(1-$ $\left.K_{32}\right) e^{-2 \delta_{n} \epsilon \tau_{2}} \pm\left(1+K_{21}\right)\left(1-K_{32}\right) e^{-2 \delta_{n} \epsilon\left(\tau_{1}+\tau_{2}\right)}$. We summarize here the main equations and parameters are given in Table 4.1 . 


\begin{tabular}{|c|l|}
\hline$k_{i}$ & $\begin{array}{l}\text { Thermal conductivity of the } i \text {-th layer } \\
\text { with } i=3 \text { corresponding to the substrate } \\
K_{i j}=k_{i} / k_{j} \\
t_{i} \\
\tau_{i}=t_{i} / a \\
\epsilon=a / b \\
\text { Thermal conductivity ratio } \\
\text { Thickness of the } i \text {-th layer } \\
\text { Relative thickness of each coating } \\
\text { Contact spot aspect ratio } \\
\delta_{n}=\frac{\beta_{n}}{4}\left[1-\frac{6}{\beta_{n}^{2}}+\frac{6}{\beta_{n}^{4}}-\frac{4716}{5 \beta_{n}^{6}}+\frac{3,902,418}{70 \beta_{n}^{8}}\right] \\
\beta_{n}=\pi(4 n+1)\end{array}$ \\
$\begin{array}{l}\text { (extremely small in our case) } \\
\text { Approximated eigenvalues of } \\
\text { the characteristic equation } J_{1}\left(\delta_{n}\right)=0 \\
\text { with } n \geq 1\end{array}$ \\
\hline
\end{tabular}

Table 4.1: Parameters of the 2 layers spreading resistance model.

Critical parameters are $t_{1}, t_{2}, k_{1}$ and $k_{2}$, the thicknesses and thermal conductivities of the first and second layer, respectively, $a$, the contact radius of the probe with the sample and $k_{3}$, the thermal conductivity of the substrate. For a Si substrate we have $k_{3}=148 \mathrm{Wm}^{-1} \mathrm{~K}^{-1}[132]$. Then, the $\mathrm{Cr}$ and BCP layer thicknesses, $t_{1}$ and $t_{2}$, have been obtained from the TEM measurements not reported here. The estimation of the contact radius of the probe is more challenging, particularly in soft materials, where the deformation of the sample surface is more likely.

By inspecting the thermal profile shown in Fig. 4.8d, we can see that as the tip is moving from the higher level (PEO) towards the edge of the PS phase, the probe voltage increases. This $13 \mathrm{mV}$ increase of the probe voltage on top of the PS matrix indicates an increase of the heat conduction from the probe to the sample, i.e., a decrease of the tip-sample thermal resistance. However, topographic artefacts have to be carefully distinguished from true variations in the voltage signal that are not related to the geometrical change in tip-sample contact area. Comparing the $20 \mathrm{~nm}$ flat area in the topography line scan in Fig. 4.8. (square red box) and the corresponding thermal signal in Fig. 4.8d, we can estimate the thermal spatial resolution is in the sub-20 $\mathrm{nm}$ range. In this region there is not cross talk between topography and thermal signals, indicating that the contact between the tip and 
the sample does not change significantly and the acquired signal is not related to topography-induced artefact. Taking into account this lateral resolution obtained from the thermal images, we estimated $a=10 \pm 2 \mathrm{~nm}$.

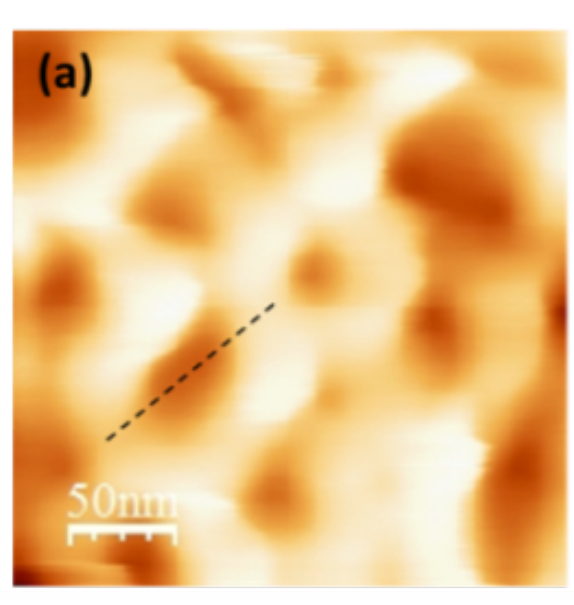

(c)

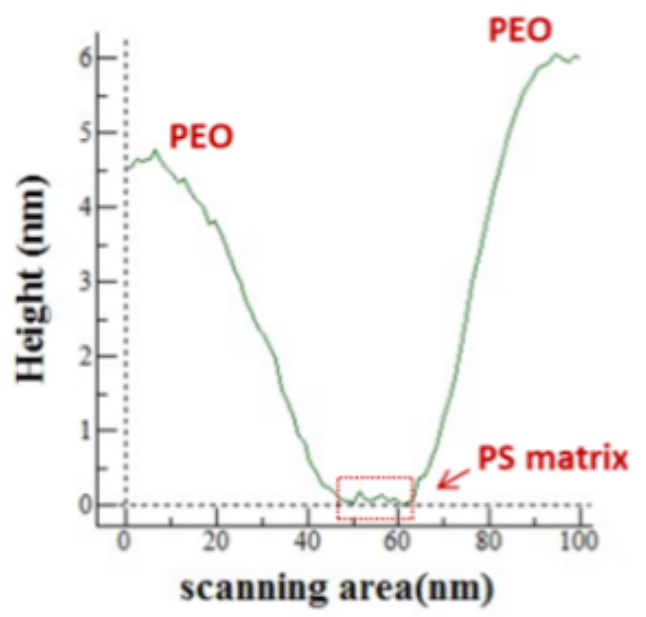

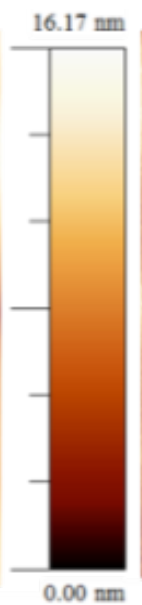

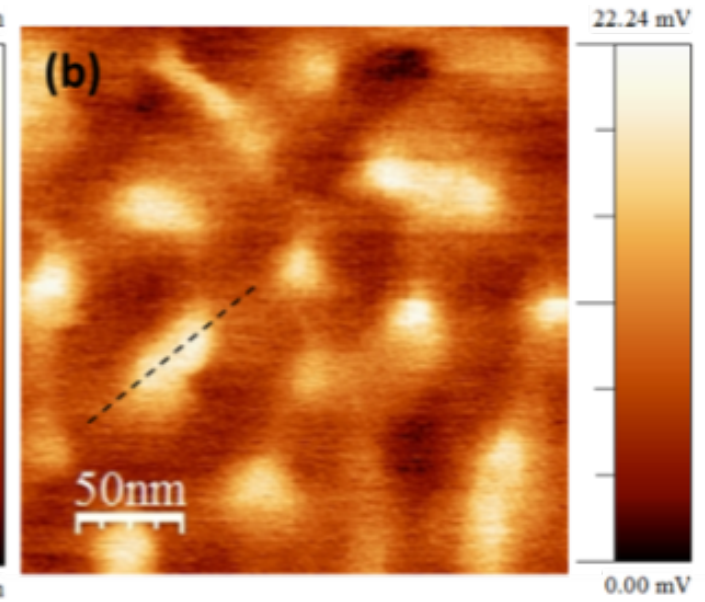

(d)

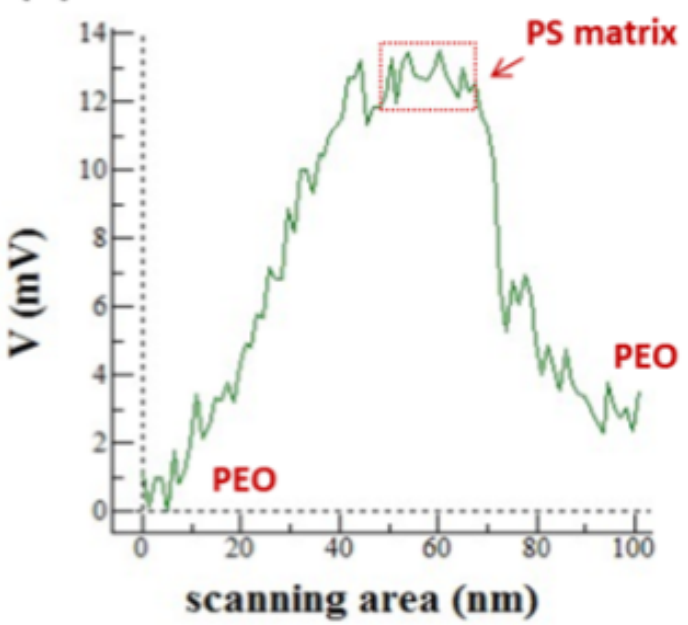

Figure 4.8: High resolution (a) topography and (b) thermal images of the modified BCP template in vacuum environment. (c) Topography and (d) voltage signal profiles along the dashed lines in the images.

For the $\mathrm{BCP}$ thin films without $\mathrm{Cr}$ the unknown parameters are $k_{2}$ and $R_{\text {int }}$ since we can estimate $R_{s p r}$ from the analytical thin-film approximation derived by Dryden 102. This estimation requires the knowledge of the thermal conductivity of the BCP films. Typical values for the individual BCP blocks are ranging from $0.1-0.17 \mathrm{Wm}^{-1} \mathrm{~K}^{-1} 146148$ for PS and $0.2-0.4 \mathrm{Wm}^{-1} \mathrm{~K}^{-1}$ for the PEO 149 . However, the intrinsic thermal conductivity of the polymers can be affected by many factors such as molecular weight 146 or confinement 150, 151. Furthermore, when subject to pressure, as it is the case under a sharp tip, the polymer thermal 
conductivity can be increased 89$]$. In the case of our BCP films, it is challenging to estimate this increased thermal conductivity. Therefore, we use a weighted average (considering the volumes of PS and PEO) thermal conductivity $k_{\text {eff }}$ and similarly, weighted average BCP film thickness $t_{e f f}$. With these values we estimated $R_{s p r}$ between $7 \times 10^{6} \mathrm{KW}^{-1}$ and $4.4 \times 10^{7} \mathrm{KW}^{-1}$. Then, we used the last unknown $R_{\text {int }}$ to reproduce the experimental data. We note that this model assumes perfect interfaces in between the layers and between the second layer and the substrate.

The measurements show that by increasing the Cr layer thickness, the thermal resistance decreases (Fig. 4.9). For the BCPs with the metal coating the unknown parameters are $k_{1}$ and $R_{\text {int }}$. By fitting these parameters to the trend observed in the experiment, we obtained $k_{1}=1.7 \pm 1 \mathrm{Wm}^{-1} \mathrm{~K}^{-1}$ as shown in Fig. 4.9 showing the total thermal resistance variations of the SThM signal. Note that we measured negligible differences in the thermal resistance between the first and the second set of samples. By analysing the thermal images and taking into account $R_{\text {int }}$ and the injected power in the probe, we estimated in percentage of the thermal resistance variations when the tip is in contact with the PEO and PS matrix (inset graph in Fig. 4.9p. We note that these values include the error derived by topography related changes of the tip-sample contact geometry and film thickness modulations. 


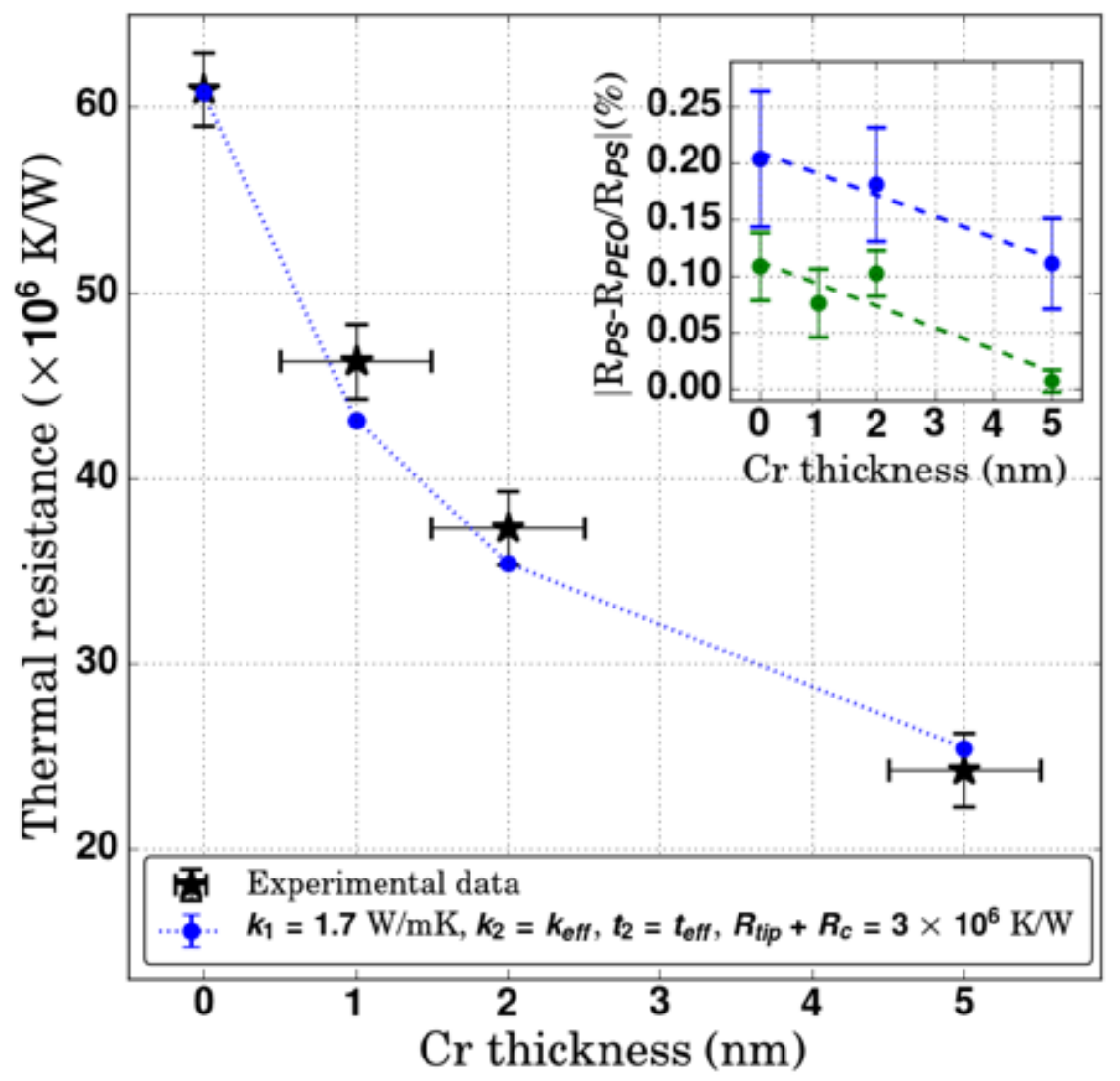

Figure 4.9: Total tip-sample thermal resistance variations as a function of the Cr thickness (black stars) and the two layers on substrate analytical model. The inset graph shows the percentage of the thermal resistance variations between PS and PEO in the BCP films with the pillar (blue spheres) and lamellae (green) morphology.

Considering the small tip apex of the thermal probe, it is reasonable to assume that the tip-sample thermal exchange is localized at the point contact. Particularly, in high vacuum experimental conditions the heat transfer through the liquid meniscus and air is eliminated and, neglecting the conductance due to radiation, the dominant heat transfer mechanism is the conduction due to the mechanical contact of the probe with the sample. Here, the heat source size is comparable to the thickness of the Cr layer and the influence of the Cr layer dominates over the substrate. Therefore the increase of the Cr layer enhances heat dissipation or conductance within the sample giving the trend seen in figure 4.9. Furthermore the decrease of the thermal resistance seen in the measurement on the sample with $1 \mathrm{~nm}$ Cr layer compared to the measurement on the pristine sample indicates that 
the heat spreading due to the presence of the metal dominates over the Cr-BCP interface thermal resistance. Finally, the fact that the total thermal resistance measured in the pristine samples without the metal coating remains the same, indicates that the intrinsic thermal conductivity of the BCP film does not change or cannot be detected after the reorganization of the block copolymer chains into aligned lamellae.

\subsection{Summary}

In this chapter, we explained the necessity and the main assumptions needed for thermal resistance measurements. Under specific conditions, SThM measurements of thermal resistance can be compared. Then, when comparison is possible, an appropriate model of the thermal resistance between the sensor and the sample heat sink is needed. This chapter demonstrated models based on spreading resistance analysis of single and double layers on a substrate as well as a simple resistances network. We first obtained quantitative values for oxide layers (silicon and aluminium oxides) demonstrating the principle of using spreading resistance models. Finally, for metal-covered block copolymers a double layer model was introduced and fitted to the experimental data.

It is worth noting that our work is based on nanoscale SThM probes in the specific measurements procedures we described. Therefore, some conclusions might not be applicable to a microscale probe such as the Wollaston wire or to different measurements procedures. For example, calibration of the SThM response $d V / V$ on the thermal conductivity could not be realised in our lab but has been reported for the Wollaston wire 73,96 and nanoscale probe 152 .

However, measurements uncertainties can arise in SThM practice as we have shown in the chapter 3 . Hence, a further increase of the SThM precision and reproducibility can be achieved by minimising experimental operations such as sample changing or probe repositioning. A sample such as the silicon oxide steps provides already a good improvement but requires to change the probe position 
for each step. In the next chapter, we present a powerful method taking the ideas reported in this chapter one step further. 


\section{Three dimensional mapping of thermal properties}

In this chapter, we demonstrate that the combination of a unique cross-sectional polishing tool and SThM provides many advantages for nanothermal investigations of buried materials properties. From isotropic and anisotropic thin films, to thermal interface materials, nanothermal properties are extracted using appropriate models.

\subsection{Introduction}

As nanomanufacturing enters commercial markets, rapid and versatile investigations of materials and devices on the nanoscale become a key requirement. Common microscopy tools reach their limit when measurement of specific physical properties at the nanoscale is needed and standard techniques cannot address challenges faced by nanofabrication processes. One of the key characteristics of materials at the nanoscale is their ability or inability to transfer heat. For example, the microelectronics industry is struggling to dissipate heat generated by hot spots in transistors arrays $[5,153$, stalling Moore's law for increase of processor speed for more than a decade. Measurements of thermal conductivity in a simple geometry such as thin films on a substrate present many difficulties to traditional techniques for layer thickness smaller than $100 \mathrm{~nm} 24$. In particular, decoupling the thermal conductivity and the effect of interface resistance between the film and the substrate, as well as between the nanoscale probe and the film, is difficult 
and is often not possible.

Furthermore, as device architecture become more complex and using three dimensional (3D) stacks, multilayer processes and 3D geometry to gain space and capacity, a new approach is required to probe thermal transport in buried layers and the interfaces. Standard thermal techniques are limited to either surface or bulk probing and cannot assess thermal transport in buried nanostructures.

Nanothermal microscopy techniques such as scanning thermal microscopy (SThM) are gaining interest as they resolve thermal properties well below the light diffraction limit 8, 154]. However, accessing intrinsic and subsurface properties remains challenging. Several groups $77,78,155,157$, have probed temperature and heat conductance in 3D using SThM. While Park et al. 78 reported measurements of ErAs/GaAs MBE superlattices with 6 nm RMS roughness, Juszczyk et al. 77] used craters in photonic structures to access subsurface materials. Cleaving devices is also possible to probe subsurface as demonstrated by Jung et al. 155 where a LED was cut to map its temperature distribution. All methods reported lack reproducibility, adaptability to different structures and most prominently ill-defined surface which create major hurdles for SThM measurements.

To address these challenges, we used a unique nano-sectioning tool using Arion beams impinging on the side of a sample at shallow angle $\left(\sim 5^{\circ}\right)$ and exiting through the sample surface, beam-exit cross-sectional polishing (BEXP) [158, 159]. The cross-sectioned surface obtained has an open angle wedge-like geometry and sub-nm surface roughness making it easily suitable for studies via standard scanning probe microscopy methods (see Fig. 5.1). Equally essential, the glancing beam-angle and inert nature of Ar create negligible surface damage and practically no modification of the measured physical properties of studied materials 158 , 159].

The BEXP process enables the measurements of the SThM response as a function of layer thickness. By analysing the SThM signal of the wedge-shaped section, we were able to extract the thermal conductivity of the layer itself by applying 
an appropriate analytical model. We confirm capabilities of this new method using standard materials and then explore buried thermal conductance of semiconductor and optoelectronic nanostructures, observing the variation in the heat conductance due to local composition and dislocation density. The ease of use of our approach renders it suitable for a broad range of samples and opens new paths for fundamental and applied research.

\subsection{Combining BEXP and SThM}

In the quest of accessing subsurface physical properties with scanning probe microscopy, we used a unique sample preparation tool. By cutting the sample at a shallow angle with Ar ion beams in BEXP configuration as represented on Fig. 5.1 a ), we create an easily accessible surface well suited for scanning probe methods as reported elsewhere 158,160 .

In the case of SThM, the BEXP procedure is particularly powerful as it enables the measurement of a sample thermal resistance as the material thickens from the substrate to the surface. Furthermore, several reports have shown the necessity in SThM experiments to measure samples with several thicknesses to deduce quantitative properties 72 74, 161]. This often requires a change of manufacturing process, preparation of special samples and does not allow measurement of the real devices. The combination of SThM and BEXP drastically reduces these hurdles. Using a single and one measurement step, the thermal resistance of various materials and thicknesses are obtained.

We demonstrate the usefulness and precision of the method on a molecular beam epitaxy (MBE) grown multilayer sample of $\mathrm{Si} / \mathrm{Si}_{x} \mathrm{Ge}_{1-x} / \mathrm{Ge} / \mathrm{Ge}_{0.9} \mathrm{Sn}_{0.1}$ that has potential use in $\mathrm{Si}$ based optoelectronics due to the potential of achieving direct bandgap in such a structure. First, a $100 \mathrm{~nm}$ Ge layer is grown on a silicon substrate. During the process, Si atoms diffuse inside the Ge layer due to the high process temperatures $[162$ and therefore create SiGe alloy of varying concentrations. Si concentration is difficult to estimate in the first few nanometers 
of the Ge layer. However Si concentration decreases with increasing thickness as diffusion does not reach $100 \mathrm{~nm}$ [163]. Then, another $100 \mathrm{~nm}$ Ge layer is grown creating a virtual Ge substrate. Finally, $200 \mathrm{~nm}$ of $\mathrm{Ge}_{0.9} \mathrm{Sn}_{0.1}$ are grown on the Ge virtual substrate. These MBE grown samples were prepared by the Institute of Semiconductor Engineering at the University of Stuttgart and interpretation of the results was realised in collaboration with Linda Haenel and Jorg Schulz.
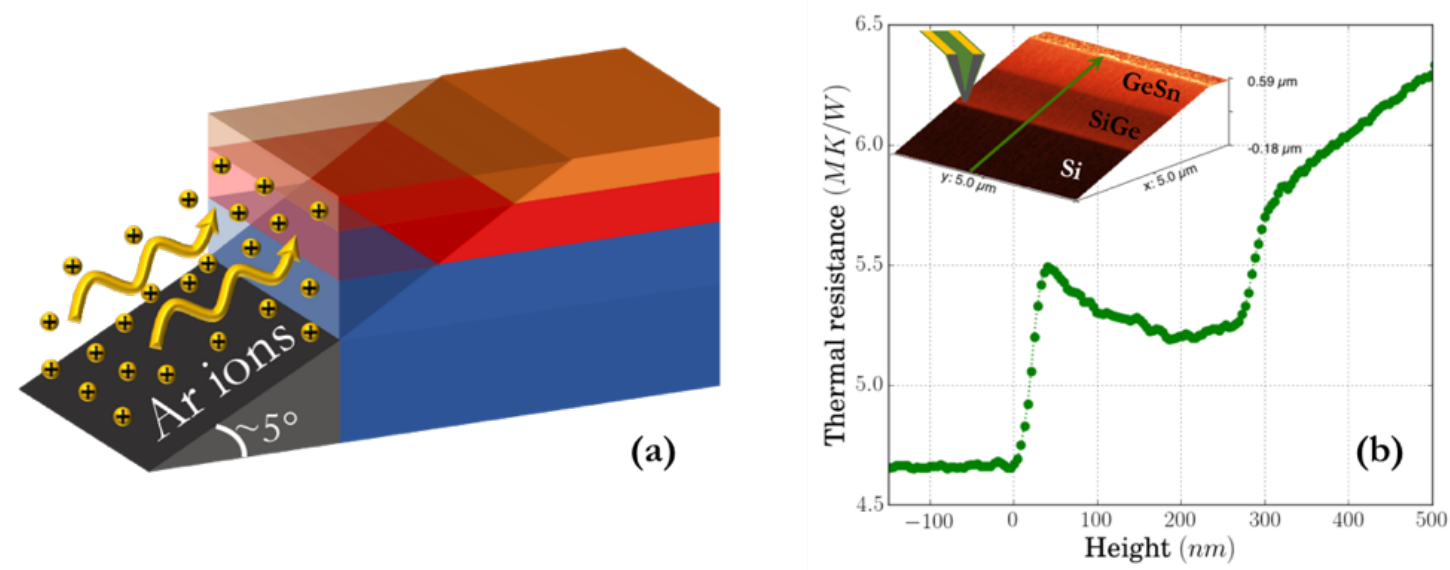

Figure 5.1: (a) Schematic beam-exit cross-section polishing (BEXP) principles. Ar ions are impinging the sample surface at shallow angle $\left(\sim 5^{\circ}\right)$ creating a SPM friendly surface. (b) Thermal resistance as a function of height starting from the first SiGe layer. Inset: 3D topography overlaid with SThM response. Arrow indicates the direction of the average thermal resistance profile.

Thermal properties of $\mathrm{Si}_{1-x} \mathrm{Ge}_{x}$ alloys have been studied extensively. Its thermal conductivity changes drastically with Ge concentration 164 and always reduces compared to the bulk values of both $\mathrm{Si}$ and Ge. When the SThM probe scans across the different layers, the thermal resistance at the tip apex will be affected by the thermal transport happening at a particular nanoscale volume in the 3D space of the sample. This transport depends on the local thermal conductance which is determined by both the local thermal conductance in the material, and for the nano-section, corresponding thickness of the subsurface layer along the z-coordinate.

Fig. $5.1 \mathrm{~b}$ shows the thermal resistance at the tip apex as a function of height inside the sample and a 3D topography representation overlaid with SThM contrast. Three regions corresponding to the silicon substrate, the virtual substrate 
and the $\mathrm{Ge}_{0.9} \mathrm{Sn}_{0.1}$ layer are observed. We can distinguish the relatively low resistance silicon substrate then a sharp increase at the transition to the $\mathrm{Si}_{1-x} \mathrm{Ge}_{x}$ layer with high density of misfit dislocations, followed by a decrease and roughly constant signal in the relaxed dislocation free Ge layer. Finally, as the tip enters the $\mathrm{Ge}_{0.9} \mathrm{Sn}_{0.1}$ layer, the heat resistance increases again.

As we will explain in the following section, the thermal spreading resistance of a layer on a substrate is expected to increase (decrease) if the thermal conductivity of the layer is smaller (larger) than that of the substrate $\left(\frac{k_{\text {layer }}}{k_{\text {substrate }}}<1\right.$ or $>$ 1). Therefore, the resistance lowering observed in the $\mathrm{Si}_{1-x} \mathrm{Ge}_{x}$ region is counter intuitive as, for any Ge content, the thermal conductivity of $\mathrm{Si}_{1-x} \mathrm{Ge}_{x}$ is always lower than that of silicon 164. However, the Ge content is varying from around $80 \%$ to $100 \%$. In this region, the thermal conductivity of $\mathrm{Si}_{1-x} \mathrm{Ge}_{x}$ is increasing.

It can be shown that in such case of an increasing layer thermal conductivity, the spreading resistance will first increase and then reduce as the thickness increases and the probe contacts more thermally conductive material. Using Finite Element modelling with a fine mesh, we can model the spreading resistance of a $40 \mathrm{~nm}$ diameter heat source on the surface. The spreading resistance is defined as

$$
R_{s p r}=\frac{T_{a v}-T_{0}}{Q}
$$

where $T_{a v}$ is the average temperature over the heat source surface and $T_{0}$ is the boundary temperature and $Q$ is the total power set on the heat source. We can then model the spreading resistance as a function of sample thickness probed. Due to the thermal conductivity profile, the material under the heat source has a gradually increasing thermal conductivity. It can be seen in Fig. 5.2 that the spreading resistance is first increasing and then reducing with increasing thickness (blue triangles), whereas with a constant conductivity, the resistance monotonously increases (red circles). 

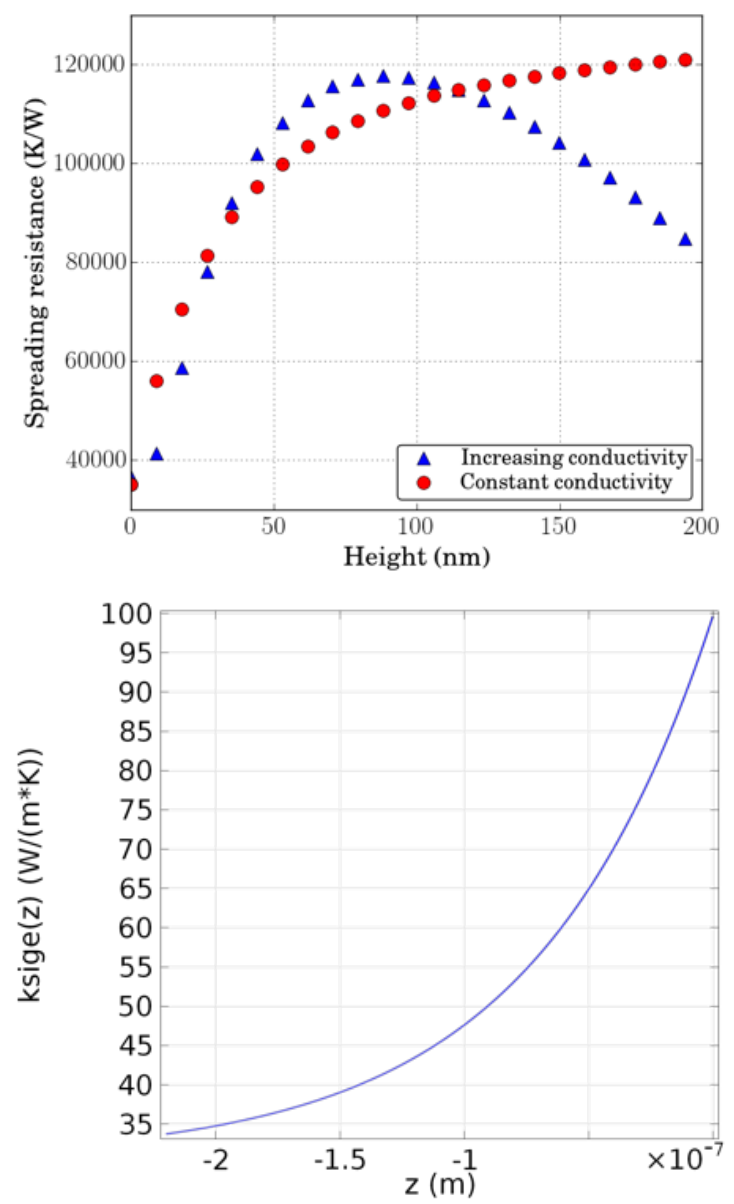

Figure 5.2: Thermal spreading resistance computed for a constant (red cirlces) and an increasing (blue triangles) layer thermal conductivity and the modelled increasing layer thermal conductivity distribution of SiGe.

This explains the trend observed in the SiGe layer and demonstrates the potential applications of the BEXP-SThM combination. It is remarkable therefore that nano-section and SThM allow the detection of variation of the local thermal conductance due to the composition and crystalline defects in the layers with a vertical resolution of around $5 \mathrm{~nm}$ in thickness.

In order to perform quantitative analysis of inhomogeneous and anisotropic gradient $\mathrm{Si}_{x} \mathrm{Ge}_{1-x}$ and $\mathrm{Ge}_{0.9} \mathrm{Sn}_{0.1}$ samples, we present an analytical model for the nanothermal characterization of ultrathin layers and confirm the accuracy of the method using well defined isotropic layered samples. 


\subsection{Quantitative measurements of thermal con- ductivity and interface resistance}

When the probe contacts the sample surface, heat is transferred to the sample and this thermal resistance from the probe to the sample is measured through the probe's electrical resistance. We can split this resistance into two main components: the interface between the sensor and the sample and the thermal spreading resistance (see Eq. 4.2
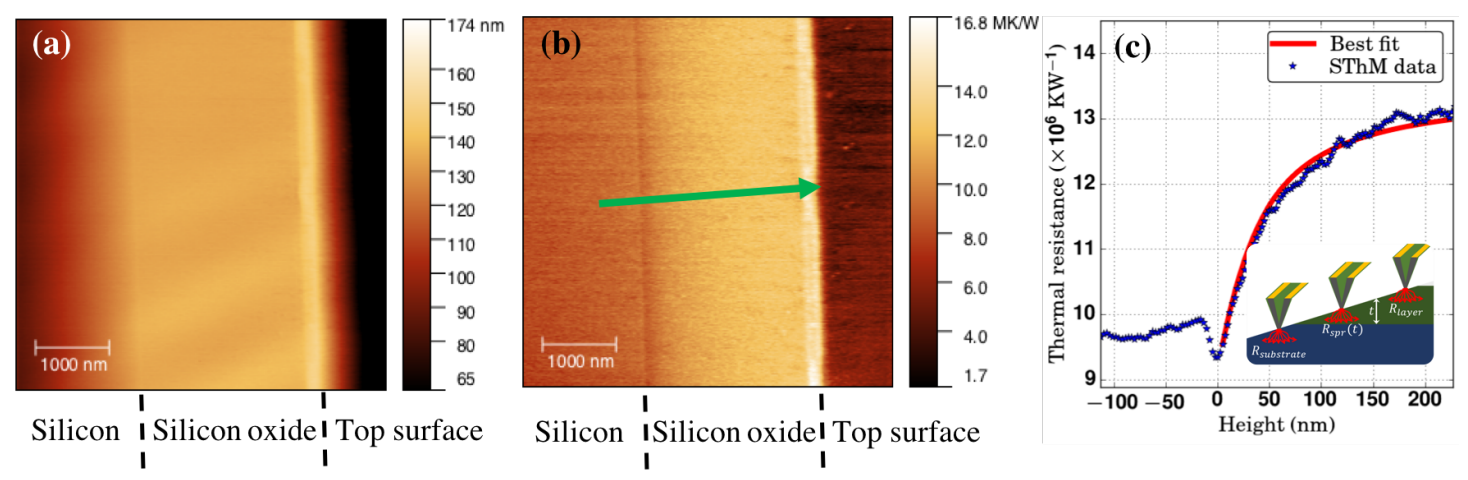

Figure 5.3: (a) Topography image of the BEXP polished $300 \mathrm{~nm}$ silicon oxide sample. The image is flattened on the oxide area. (b) Total thermal resistance map of the $300 \mathrm{~nm}$ silicon oxide on silicon. (c) Average profile along the green arrow direction in (b) fitted with spreading resistance model. Inset is a schematic view of the SThM scanning along the polished sample with increasing thickness.

The thermal spreading resistance depends on the structure and materials composing the sample. In the simple case of a bulk isotropic material, it is given by:

$$
R_{s p r}=\frac{1}{4 k a}
$$

with $k$ the thermal conductivity of the material and $a$ the effective contact radius between the probe and the sample. This resistance corresponds to the substrate response as represented on the inset of Fig. 5.3.

In the present case, we are dealing with a single layer on a substrate. Therefore, we use a model taking into account the heat spreading in the layer resting on the substrate as well as the interface resistance between the layer and the substrate. Such model derived by Muzychka et al. [105, 107] was already introduced (see 
Eq. 4.4). For thin films and nanoscale systems, diffusive models might not be representing the exact physical nature of hte heat transfer. For example, ballistic transport might become relevant. However, this spreading resistance model offers a good framework of understanding and provides useful discussion tools. Thus, the quantities extracted are therefore effective quantities and might not be the physical ones.

To quantify thermal properties such as the thermal conductivity, we investigated standard materials used in various places across the semiconductor industry 165, 166]: $300 \mathrm{~nm}$ silicon dioxide, $60 \mathrm{~nm}$ spin-on carbon and $10 \mathrm{~nm}$ spin-on glass. All layers are resting on a Si substrate. These materials provide a case study and a test system demonstrating principles of the techniques.

After polishing at shallow angle, we obtained a smooth surface easily accessible by our thermal probe. The thermal signal received accounts for the thermal resistance from the probe to the sample. With an appropriate model explained in Chapter 3, we can link this signal to the thermal resistance at the tip apex. This model takes into accounts major heat channels of the system: conductance through the cantilever to the microscope, heat dissipation to the environment through the air and heat transfer through the air gap to the sample. It also includes the geometrical characteristic of the heater shape which creates a different temperature gradient in or out of contact.

To confirm the accuracy of the measurements performed in air, we compared experiments performed in air and vacuum on the same sample (see Fig. 5.4). Similar results were obtained and features observed on the surface. We note a slightly better sensitivity in vacuum which can be attributed to a smaller effective contact area, as expected in vacuum. However, the good agreement between both measurements supports the measurements performed in air as the air thermal resistance variations don't affect the profile measured. 


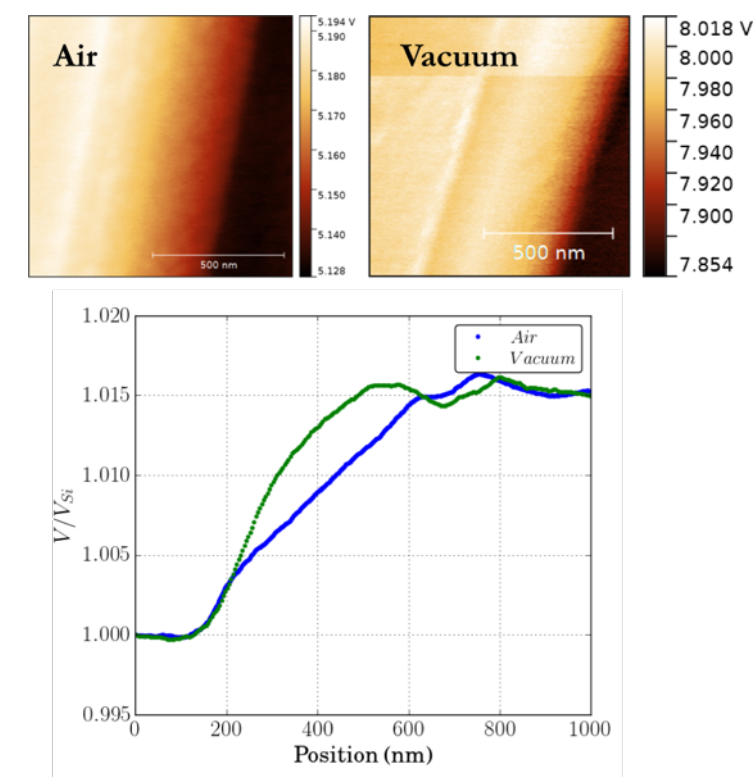

Figure 5.4: Comparison of air and vacuum measurements of the same sample. Top: thermal maps of the same sample in air and vacuum. Bottom: SThM profiles normalized to the substrate and top surface measurement.

Fig. 5.3 a-b show the topography and thermal resistance maps of a $300 \mathrm{~nm}$ thermal oxide on silicon. Silicon response is lower than that of silicon oxide as expected. At the transition between the polished area and the top surface, thermal resistance is varying non-uniformly. This can be attributed to the change of contact area as the angle between the probe and the sample is changing. In Fig. $5.3 \mathrm{k}$, a profile was taken along the green arrow direction of Fig. $5.3 \mathrm{~b}$. This profile is averaged perpendicularly to the arrow direction. An increase of the thermal resistance is obtained with increasing oxide thickness corresponding to an increasing spreading resistance. At the silicon-oxide interface, a dip in the signal can be observed. This can be attributed to the change of the angle due to different polishing $\left(<2^{\circ}\right.$ different $)$ or change of the interface resistance between the apex and the surface.

When the probe is solely in contact with the oxide layer, we can assume that this interface resistance remains constant within the whole layer. In other words, $R_{\text {int }}$ of Eq. 4.2 is constant for the same material. Then we can apply the analytical model describe above (Eq. 4.4) and using unknown parameters as fitting parameters. There are four unknowns in such system: the interface resistance between 
the tip apex and the sample, the effective contact radius, the layer thermal conductivity and the interface thermal resistance between the layer and the substrate. With an appropriate fitting function, as described hereafter, we can remove the tip-sample interface resistance as it is considered constant and deduce its value afterwards.

Eq. 4.4 has a major unknown, typical for any SThM conductance measurement: the effective interface resistance between the probe and the sample, $R_{\text {int }}$. The second terms, $R_{s p r}$ depends on the sample structure and materials and is often the target of the experiment. In our case, the spreading resistance is a function of the thickness $R_{s p r}=R_{s p r}(t)$ and we assume that $R_{\text {int }}$ remains constant for the same material. To remove the contribution of $R_{i n t}$ in the experimental data, we can define a new fitting function accounting only for the spreading resistance effect:

$$
f(t)=R_{X}-R_{X_{0}}=R_{s p r}(t)-R_{s p r}\left(t_{0}\right)
$$

where $t_{0}$ is a given reference thickness. Using $f(t)$ to fit the data removes temporarily $R_{\text {int }}$ as all variations in the data come from the spreading resistance variations only. $R_{\text {int }}$ can be obtained afterwards by simply finding the offset to match the measured resistance.

Then, we can use the $300 \mathrm{~nm}$ oxide sample as a calibration sample for the effective contact radius. By assuming literature values for the thermal conductivity $\left(k_{\mathrm{SiO}_{2}} \approx 1 W / m K 143,167\right)$ and the interface thermal resistance between silicon oxide and silicon $\left(r_{\text {int }_{\mathrm{Si}_{-S i O}}} \approx 1 \times 10^{-9} \mathrm{~m}^{2} \mathrm{~K} / W[143,168]\right)$, we are left only with the effective contact radius. With these parameters, we obtained $a=56.1 \mathrm{~nm}$. We note that the fit quality (see Fig. 5.3.) is reasonable.

Applying the same method and using the calibrated contact radius, we could measure thermal conductivities and the interface resistances of $60 \mathrm{~nm}$ spin-on (SO) carbon and $10 \mathrm{~nm}$ spin-on glass. Results are displayed in Table 5.1. We note a general coherence in the data obtained as spin-on carbon is expected to be more thermally conductive than spin-on glass. Experimental data and fitted curves are 
shown on Fig. 5.5.

\begin{tabular}{|c|c|c|c|}
\hline Fitting parameters & $300 \mathrm{~nm} \mathrm{SiOx}$ & $60 \mathrm{~nm} \mathrm{SO}$ carbon & $10 \mathrm{~nm}$ SO glass \\
\hline$k_{\text {layer }}\left(\mathrm{Wm}^{-1} \mathrm{~K}^{-1}\right)$ & $\mathbf{1}$ & $0.8 \pm 0.1$ & $0.3 \pm 0.1$ \\
$r_{\text {int }}\left(\mathrm{m}^{2} \mathrm{~K}^{-1} \mathrm{~W}^{-1}\right)$ & $\mathbf{1} \times \mathbf{1 0}^{-\mathbf{9}}$ & $4 \pm 2 \times 10^{-9}$ & $2 \pm 2 \times 10^{-9}$ \\
$R_{\text {int }}\left(\mathrm{KW}^{-1}\right)$ & $9.0 \pm 0.1 \times 10^{6}$ & $5.3 \pm 0.1 \times 10^{6}$ & $6.0 \pm 0.2 \times 10^{6}$ \\
$a(\mathrm{~nm})$ & 56.1 & $\mathbf{5 6 . 1}$ & $\mathbf{5 6 . 1}$ \\
\hline
\end{tabular}

Table 5.1: parameters obtained by fitting equation 4.4 to experimental data. For silicon oxide, literature values are used to obtain $a$ and $R_{\text {int }}$. Bold values are assumed from literature and italic value is derived from the $\mathrm{SiO}_{2}$ calibration.
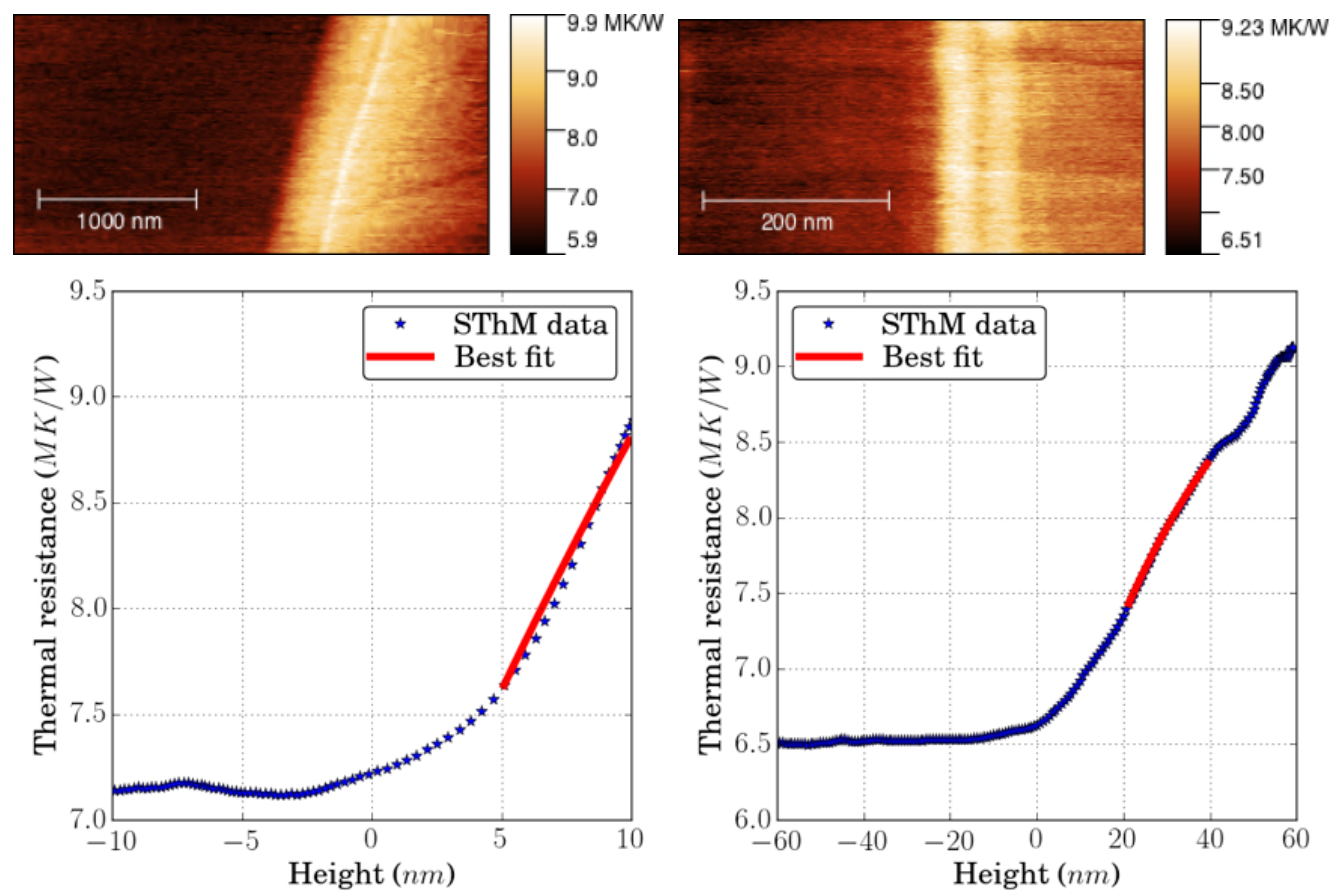

Figure 5.5: Thermal maps and resistance profiles for the $60 \mathrm{~nm}$ spin-on carbon (left) and $10 \mathrm{~nm}$ spin-on glass samples (right). The fitted data is shown in red.

\subsection{Thermal transport in anisotropic media}

The discussion above is valid for isotropic and diffusive systems of layers on a substrate. In some situations, this is not applicable when for example, thermal conductivity is changing with the thickness or when ballistic transport becomes important. In this section, we investigate nanoscale transport in anisotropic systems. First, we qualitatively compare thermal conductivities in anisotropic layers. 
Using FEA, we then quantify the thermal conductance of gradient anisotropic nanolayered materials.

An example of anisotropic system was shown in Fig. 5.1. Carrying on these multi-layered structures, we investigate 2 samples having similar composition but different processing. It was demonstrated that GeSn alloys are metastable 169 171 and their crystal quality can be altered upon annealing at high temperature 172. Sn mobility inside the Ge can increase drastically with temperature and tend to form clusters and segregate 170 . To assess these effects, we compared two samples: one sample was BEXP cross-sectioned as grown and the other one was annealed at $500^{\circ} \mathrm{C}$ prior cross-sectioning and characterization.

Figure 5.6 shows the thermal resistance for these two samples. Note that the resistance was normalized both to $\mathrm{Si}$ and Ge. The increase and further decrease found in the $\mathrm{Si}_{1-x} \mathrm{Ge}_{x}$ region was already discussed in the introduction and is attributed to the reduction of Si concentration in Ge. We can observe a slight difference in the peak of the $\mathrm{Si}_{1-x} \mathrm{Ge}_{x}$ region. The annealed sample is less resistive than its as grown counterpart. This striking result assesses the effect of annealing on the alloy structure. Upon annealing, $\mathrm{Si}_{1-x} \mathrm{Ge}_{x}$ reorganisation occurs and results in a less resistive media.

In the pure Ge region, we obtained an almost flat response which indicates that the spreading resistance isn't affected by the increase of Ge layer thickness. Then, when entering the $\mathrm{Ge}_{0.9} \mathrm{Sn}_{0.1}$ layer, the resistance increases for both samples. This can be expected because of the lower thermal conductivity of GeSn alloys 173 . 174] (between 1 and $10 \mathrm{~W} / \mathrm{mK})$ compared to pure Ge $\left(\sim 20 \mathrm{Wm}^{-1} \mathrm{~K}^{-1}\right.$ for 100 nm film 173, 175]).

However, when looking at the derivative of the thermal resistance in this layer, a difference can be observed between the as-grown sample and the annealed one. The derivative value is higher, for the annealed sample. Therefore, the spreading resistance is increasing faster in the annealed sample indicating a lower thermal conductivity of the annealed GeSn layer compared to the as grown sample. Simi- 
larly to the $\mathrm{Si}_{1-x} \mathrm{Ge}_{x}$ region, we can explain this result by a different GeSn crystal quality. As it is expected, annealing is likely to create clusters of Sn inside the Ge. These aggregates act as phonon scattering elements, hence reducing the thermal conductivity.

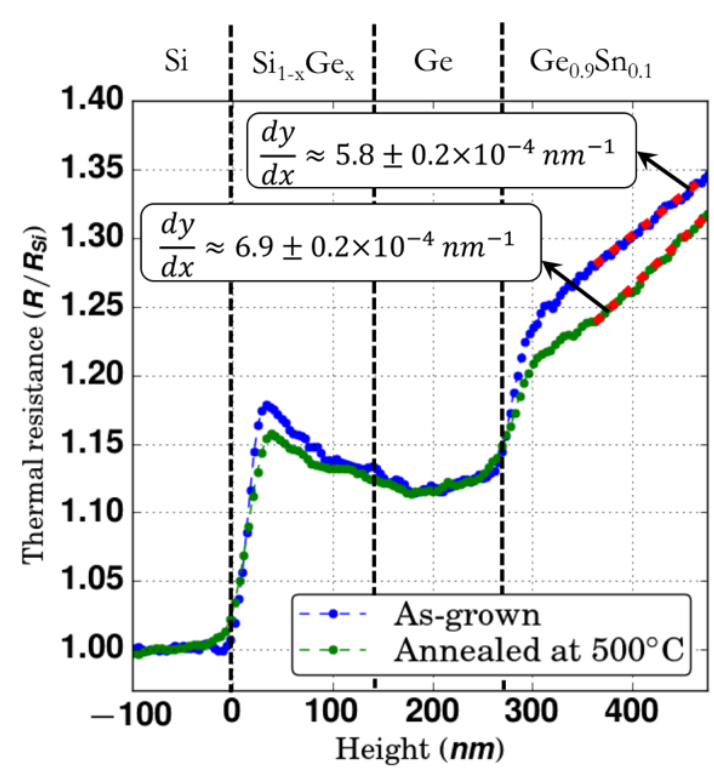

Figure 5.6: Thermal resistance as a function of height for as-grown (blue) and annealed (green) samples. Different slopes of the GeSn region are indicated and are possibly due to different crystal quality in this region.

Modelling anisotropic systems remains challenging especially in cases where ballistic transport becomes important as solutions to Boltzmann transport equation need to be found. We limit the discussion to diffusive systems and address the question of thermal conductivity anisotropy using finite element analysis. Diffusive transport assumption remains valid if the heat source dimension a is bigger than the carrier mean free path (MFP), $l$. That is if the system Knudsen number $(K n=l / a)$ is smaller than $1(K n<<1)$. In our system, phonon MFP is greatly reduced compared to bulk values due to interface scattering and impurities 164. Except for Si where $l \sim 300 \mathrm{~nm}[15]$, MFPs are smaller than $100 \mathrm{~nm}$, the effective contact radius 173 .

To further investigate the anisotropy effect, we cross-sectioned a $\mathrm{Si}_{1-x} \mathrm{Ge}_{x}$ sample with a gradient composition of Ge. The layer was grown on a Si substrate with a Ge concentration going from $0 \%$ at the bottom to $23 \%$ at the top surface, 220 
$\mathrm{nm}$ away from the substrate. We opted to model this sample as a series of steps as sharp angles increase dramatically the computation time. In this model, we assumed a linear increase of the Ge content $\left(x_{G e}\right)$ with the height inside the layer $(t)$ : $x_{G e}=t \frac{0.23}{220}$. We chose an analytical expression for the alloy thermal conductivity from the literature $176-178$ and implemented it in our Finite Element model as shown on Figure 5.7. This sample of gradient $\mathrm{Si}_{1-x} \mathrm{Ge}_{x}$ alloy on a silicon substrate was grown by collaborators from ICN2 in Barcelona.
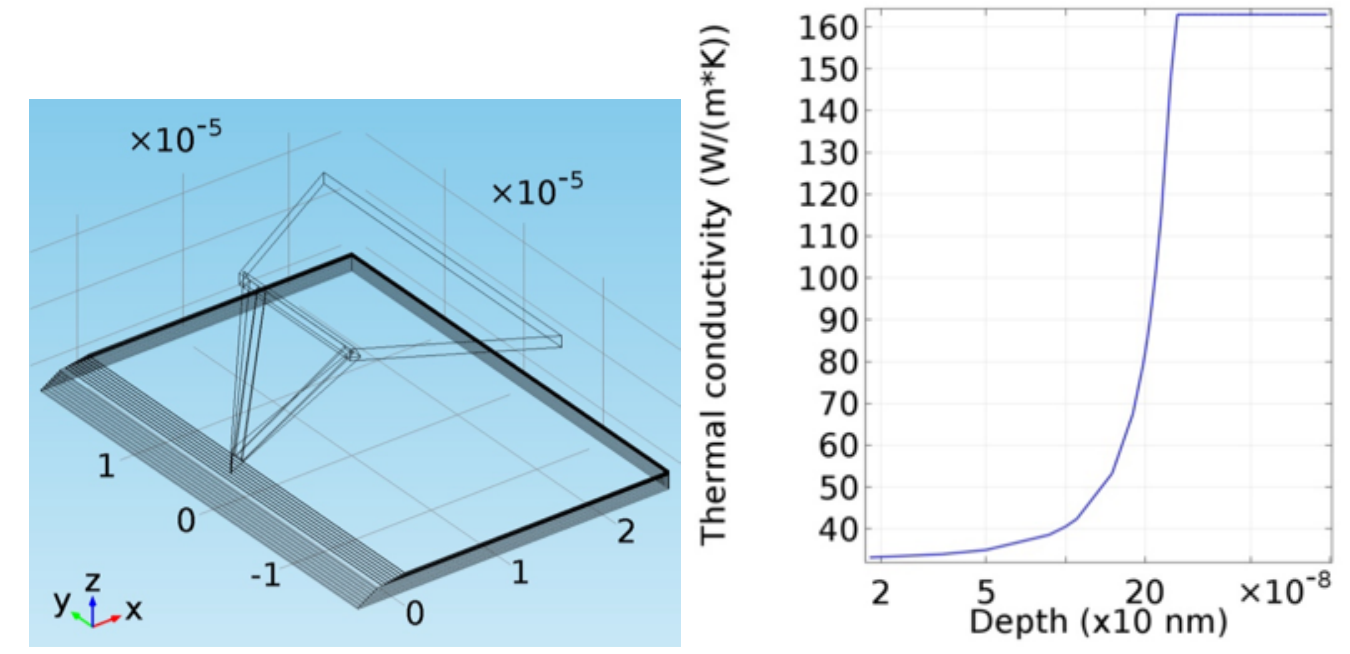

Figure 5.7: Left: Finite Element model geometry with several steps mimicking the cross-sectioned surface. Right: thermal conductivity profile across the model steps

Fig. 5.7 shows the thermal conductivity inside the material from top to bottom set as material property for the first $220 \mathrm{~nm}$. On the top of the layer, the thermal conductivity is that of $\mathrm{Si}_{0.97} \mathrm{Ge}_{0.23}$ and as we go deeper inside the steps, it is increasing toward the bulk Si thermal conductivity value.

In Fig. 5.8, results of the modelling are compared directly to experimental results. The thermal resistance measured from the SThM tip is normalized to $\mathrm{Si}$ thermal resistance for the comparison purpose. The thermal resistance measured here accounts for both contact (interface) thermal resistance between the tip and the sample and the spreading resistance inside the material. We modelled the probe behaviour on different steps corresponding to different heights from the silicon substrate and compute the corresponding thermal resistance. This thermal 
resistance was also normalized to the Si substrate.

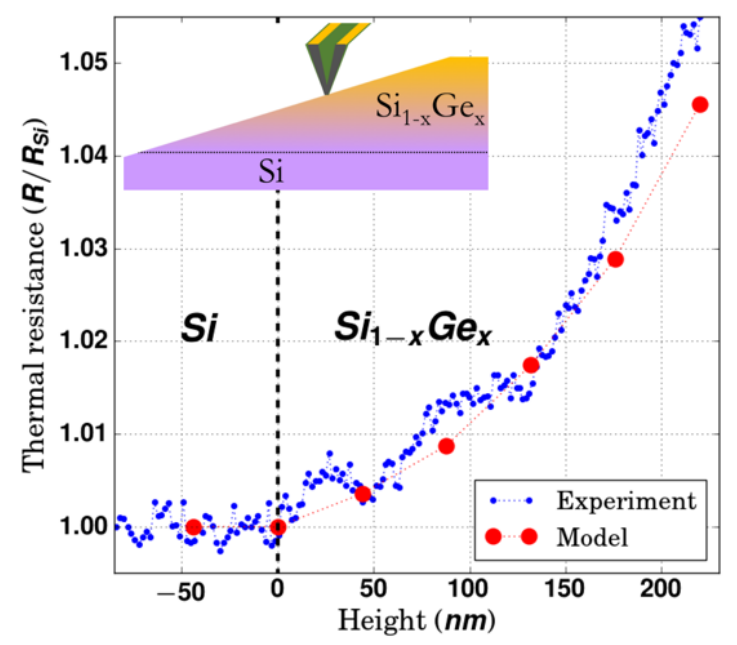

Figure 5.8: Polished $\mathrm{Si}_{1-x} \mathrm{Ge}_{x}$ on silicon sample. Modelled and measured thermal resistance as a function of height and thus increasing Ge composition are compared.

Few comments can be made on the comparison between the model and experiments. First, the observed increases in thermal resistance are similar and can be explained mainly by the distance to the substrate and reduction of thermal conductivity. Then, when reaching the top of the layer, a deviation is seen that can be connected wuth the following reasons. Ge concentration is not known precisely and composition measurements are required for a more detailed knowledge of the Ge content. It is also possible that the Ge content does not increase linearly inside the layer and that some Ge accumulated at the top. Finally, we model thermal conductivity using bulk thermal conductivity values whereas this value can be greatly reduced in thin film 164, 175.

\subsection{Characterisation of Thermal Interface Mate- rials}

Thermal interface materials are used in industry for heat management purposes. They usually help to control over heating in devices by interfacing a hot side to a cold heat sink. In thermal interfaces materials (TIM) developments, carbon nanotubes have attracted numerous studies and prototypes have been realised 
using CNTs relying on their high thermal conductivity. In this section, we study TIM morphology and nanoscale thermal properties using several systems. All TIM investigated here are formed by CNTs grown on a silicon substrate and capped by a polymer layer. This polymer layer is usually covered by another material either for protection or for serving as a heat bath. The samples were prepared at Thales by Odile Bezencenet and collaborators.

First, we used the nano-cross-sectioning BEXP approach described before to unveil buried morphology of the TIM. SEM and UFM were used to describe the nanotubes arrangement and the penetration of the polymer inside the nanotubes layer. Then, combining SThM with various sample preparation techniques, we attempted to study nanothermal properties of TIMs. This last section, even if not fully conclusive, provides a strong background for further nanothermal studies of TIMs.

\subsubsection{Buried morphology and structure}

Internal structure of thermal interface materials is always a major unknown when measuring their physical properties. Interfaces preparation involving several manufacturing steps changes the morphology of its constituents and a precise knowledge of the materials between either side is crucial for improvement.

A common challenge faced when using carbon nanotubes for efficient thermal interfacing is the contact resistance at both ends. It was shown that using a capping polymer layer can significantly improve the thermal coupling between the nanotubes and a copper heat $\sin k 179$. However, control of the CNTs penetration inside the polymer layer is needed to study the coupling efficiency.

Standard cross-sectional tools such as Focused Ion Beam (FIB) and TEM investigations requires long and costly preparations. We therefore used BEXP which used shallow angle to create a smooth wedge-like layer suitable for any standard AFM or SEM technique as explained earlier. This technique also has the advantage, compared to other cross-sectioning tools, to unveil internal morphology over 
a wide area of few $\mathrm{mm}^{2}$, instead in a small region of few $\mu \mathrm{m}^{2}$. Therefore inhomogeneity can be assessed at various places. This is a major bonus as inhomogeneities are likely to differ from one place to another.

To characterize the nano-cross-sectioned TIM, we used SEM imaging and UFM to map nanomechanical properties and gain insights on the internal morphology of the interface. In this case, the interface consists of vertically aligned CNTs on a seed layer and capped with a polymer layer covered by a silicon oxide thin film for protection. SEM images are displayed on Figs. 5.9. First, we observe a high heterogeneity within the nanotubes layer (Fig. 5.9a). The nanotubes that were vertically aligned in the previous section appear mixed with different orientation (Fig. 5.9d and 5.9 ). This can be attributed to the manufacturing process. When the vertically grown nanotubes are covered, due to their relative low bending strength, they can start folding. This is clearly seen on Fig. 5.9k which shows the first part of the nanotubes on top of the seed layer. The tubes almost appear as lying on the substrate, thus showing high bending in this region. 


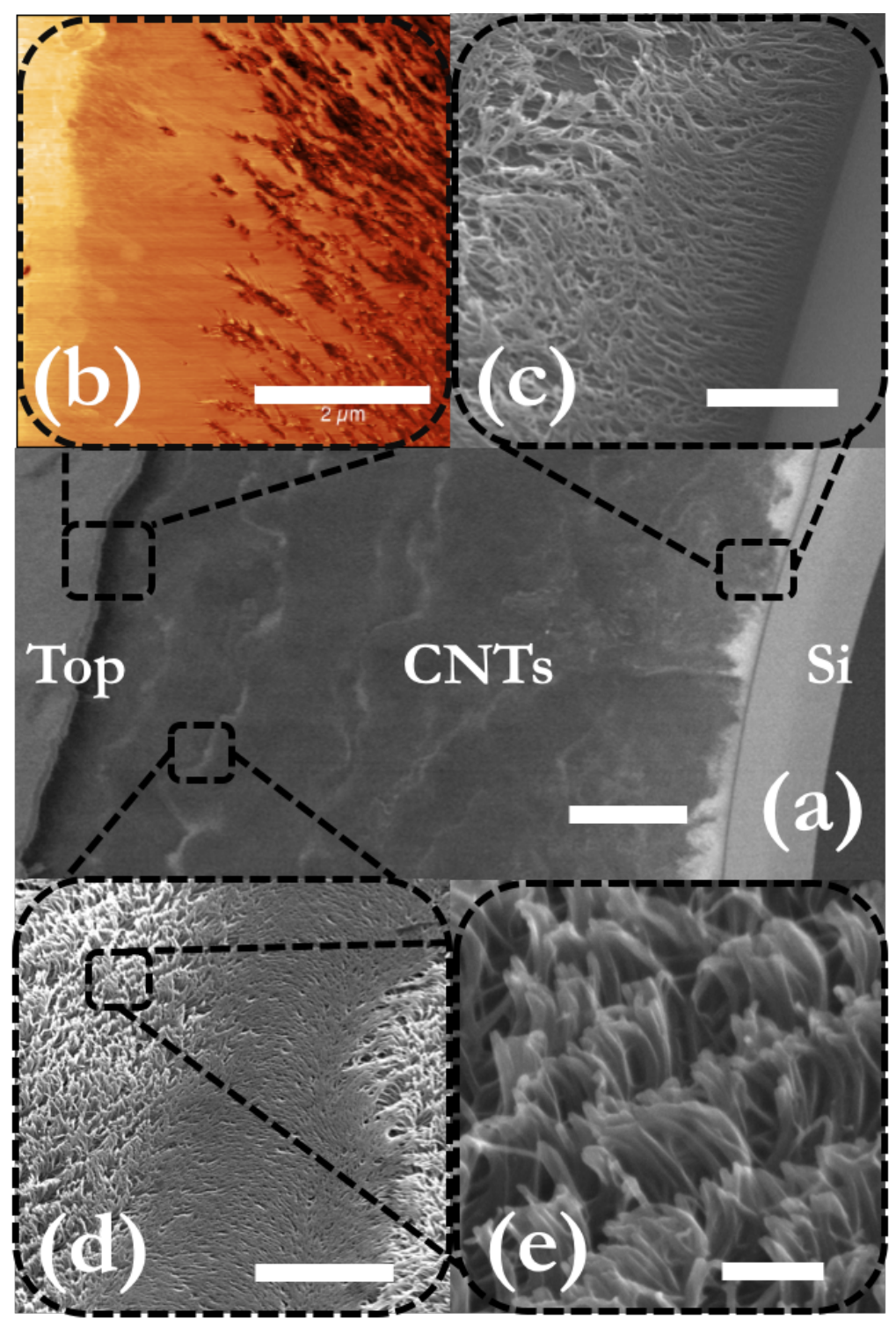

Figure 5.9: Images of the full interface polished with BEXP. (a) Large view of whole layers, from left to right: capping oxide, polymer, CNTs, seed layer, silicon, scale bar is $50 \mu \mathrm{m}$. (b) UFM image detailing the CNTs-polymer mixing, scale bar is $2 \mu \mathrm{m}$. (c) Detail of the seed layer-CNTs interface, scale bar is $2 \mu \mathrm{m}$. (d) Detail of CNTs region highlighting different CNTs orientation, scale bar is $5 \mu \mathrm{m}$. (e) Detail of CNT bundles. Scale bar is $500 \mathrm{~nm}$.

Finally, on Fig. 5.9p, we imaged the interface between the polymer layer and the nanotubes. This interface appears to span around several microns laterally, We imaged this region with UFM to highlight the presence of polymer within the nanotubes. The different layer composing the top of the TIM can be observed. Especially, the polymer layer is observed penetrating the nanotubes at least 300 $\mathrm{nm}$ below the capping layer. This is observed on Fig. 5.10 where a 3D topography 
image is overlaid with UFM contrast. Stiffer material appears brighter. Below the capping layer, the polymer forms an interface with the nanotubes ends. The latter appears interspersed with the polymer layer over several 10s of nanometers. As such, the polymer layer is expected to produce a good thermal contact with the nanotubes layer and enhanced the TIM efficiency.

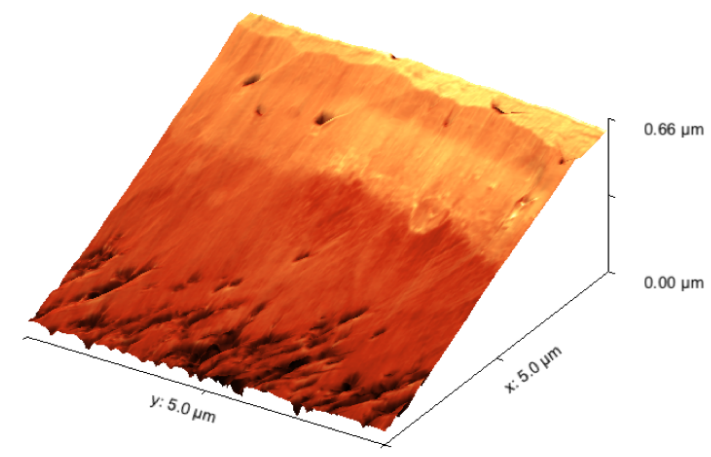

Figure 5.10: 3D topography overlaid with UFM signal.

\subsubsection{Nanoscale thermal mapping of full interface}

After inspecting the internal morphology of the thermal interface, we turn to fully assembled TIM structures. A full TIM is usually composed of two sides thermally connected with composite materials, in this case nanotubes. As nanotubes are grown on a silicon substrate, it serves as one heat bath of the TIM. On the other side, as we saw, a polymer layer is covering the top of the nanotubes for increasing conductance and usually capped with an extra layer for a second heat bath. As we already described, the nanotubes loose their vertical alignment and they form big clusters during this fabrication process. The impact of the manufacturing on the nanothermal properties is a key interest for the TIM efficiency improvement.

In this last section, we attempt to explore these nanothermal properties using three different approaches. As 3D probing and access to buried interfaces are required, all approaches involve a sample preparation step that will be described. First, we used a similar polishing tool as for BEXP but in this case, the polishing angle was set at $\sim 90^{\circ}$ to obtain a perpendicular cross-section. The second sample was prepared using the same process as for a TEM characterization. Finally, the 
third TIM was polished on one of its edges using FIB. Each sample fabrication method offer advantages and disadvantages that we describe. SThM measurements on each sample were not fully conclusive being affected by the tip-sample contact area. However, this work provides a good test bed for future TIM characterisation.

\section{Perpendicular BEXP}

The sample used for perpendicular BEXP was as described above with a copper heat sink over the polymer layer. Fig. 5.11 represents the sample and how it is mounted for SThM characterisation. Note that as there was more material to be polished than in traditional BEXP, the process took around three times longer ( $\sim 15$ hours). However, the resulting surfaces were smooth compared to other methods described later.

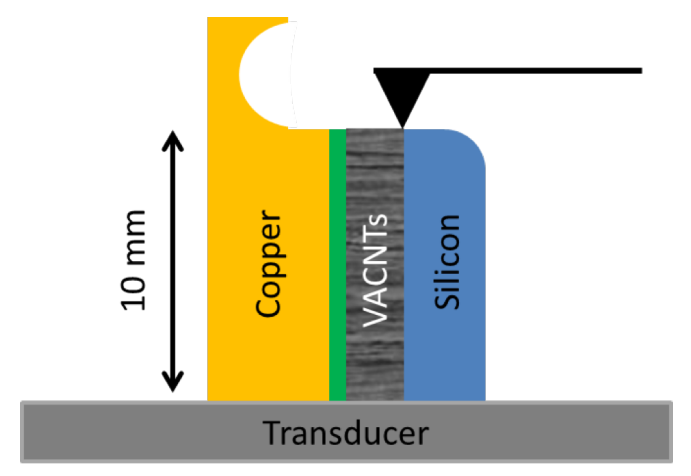

Figure 5.11: Perpendicular BEXP schematic, not to scale. The sample is mounted on its edge and the polished side is accessible with the SThM probe.

We observed a dip between the polymer layer and the copper that could have the following origins (see Fig. 5.12). First, from the TIM fabrication, it is possible that the copper and polymer layer were not fully in contact, especially on the sample where we performed the polishing. This is supported by the depth of this gap which was measured to be at least $400 \mathrm{~nm}$ with AFM whereas the space between the polymer and the copper was around $2 \mu \mathrm{m}$ large.

The other explanation could be that the perpendicular cross-sectioning impacts the interface between the polymer and the copper. As Ar ions impinges the sample from the silicon side, the polymer is removed first, followed by the copper. The 
polymer is expected to be easily polished by these nearly $90^{\circ}$ incident angle ions rays while the copper, formed of heavy atoms will be less affected. Therefore, the ions rays, after having removed the polymer materials strike the heavy copper atoms and could then deflect, or even heat the copper, and affect the polymer below. This way, a dip could be formed at the copper-polymer interface.

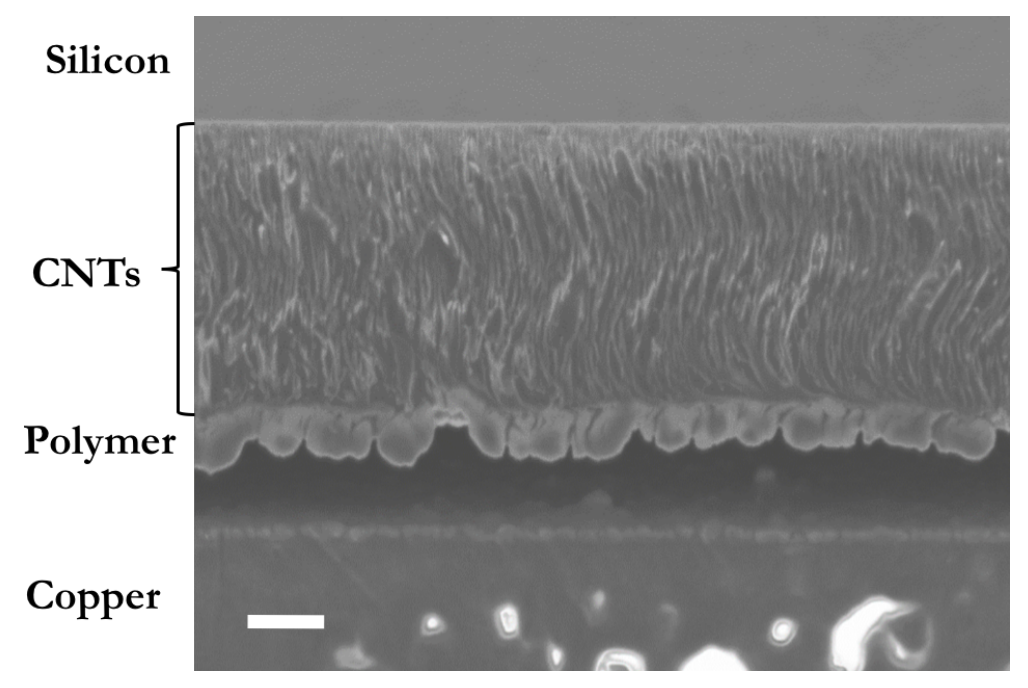

Figure 5.12: SEM image of the full TIM as obtained from perpendicular nanocross-sectioning. The scale bar is $2 \mu \mathrm{m}$. The dip between the polymer and copper is observed.

We performed SThM measurements in ambient environment (see Fig. 5.13). The top of the SThM scan shows a small region of the silicon substrate and at the bottom we observe the polymer layer. The former appears more thermally conductive (darker) than the latter, as expected from the bulk thermal conductivity of these materials. Aside from the disorder already observed in the CNT layer, various SThM signals are received. It is hard to distinguish the components of this signal as topography artefacts are likely to dominate due to the highly inhomogeneous surface of the CNTs. 


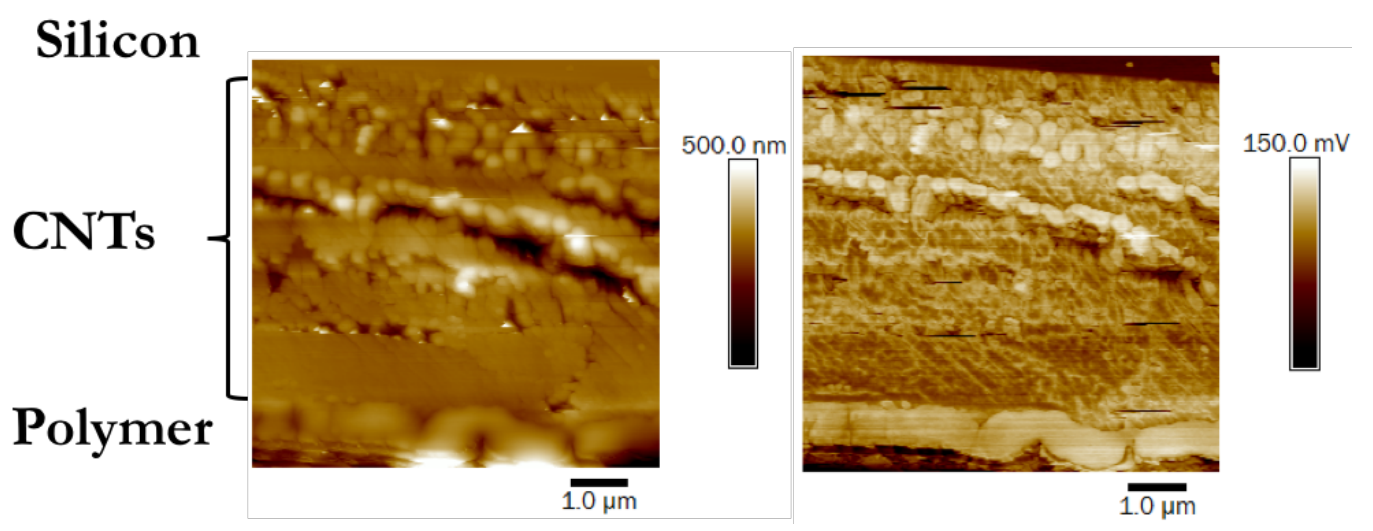

Figure 5.13: Topography (left) and SThM (right) maps of the BEXP perpendicularly polished sample.

\section{TIM sample section via TEM preparation procedure}

In this second sample, a TEM sample preparation method was used. A small piece of the material is isolated using precise manipulation systems. Then it is encased in a platinum frame and attached to a grid/holder as shown on Fig. 5.14. Ambient SThM setup was also used here for thermal characterisation. We remark that this very fragile size of the sample, surrounded by voids, was creating many challenges for performing experiments. 


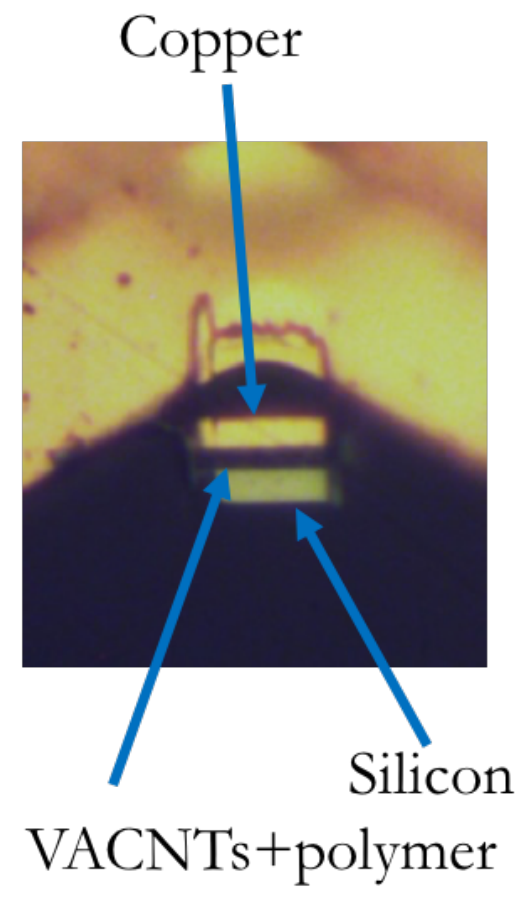

Figure 5.14: Optical microscope image of the TIM sample prepared for TEM imaging.

Results are displayed on Fig. 5.15. All layers appear clearly on the SThM signal. We observe a general decrease in the signal from a high silicon response to a low copper one. We attributed this observation to the different air volume surrounding the SThM probe at each position. When the probe is in contact with the silicon, on the outer part of the TEM section, the effective air volume connecting the probe to the sample is smaller than when the probe contacts the copper where the whole probe body and sensor are close to the TEM section holder. Therefore more heat is transferred to the sample when scanning the copper and polymer whose response appear more conductive than the silicon, which is highly unlikely. 


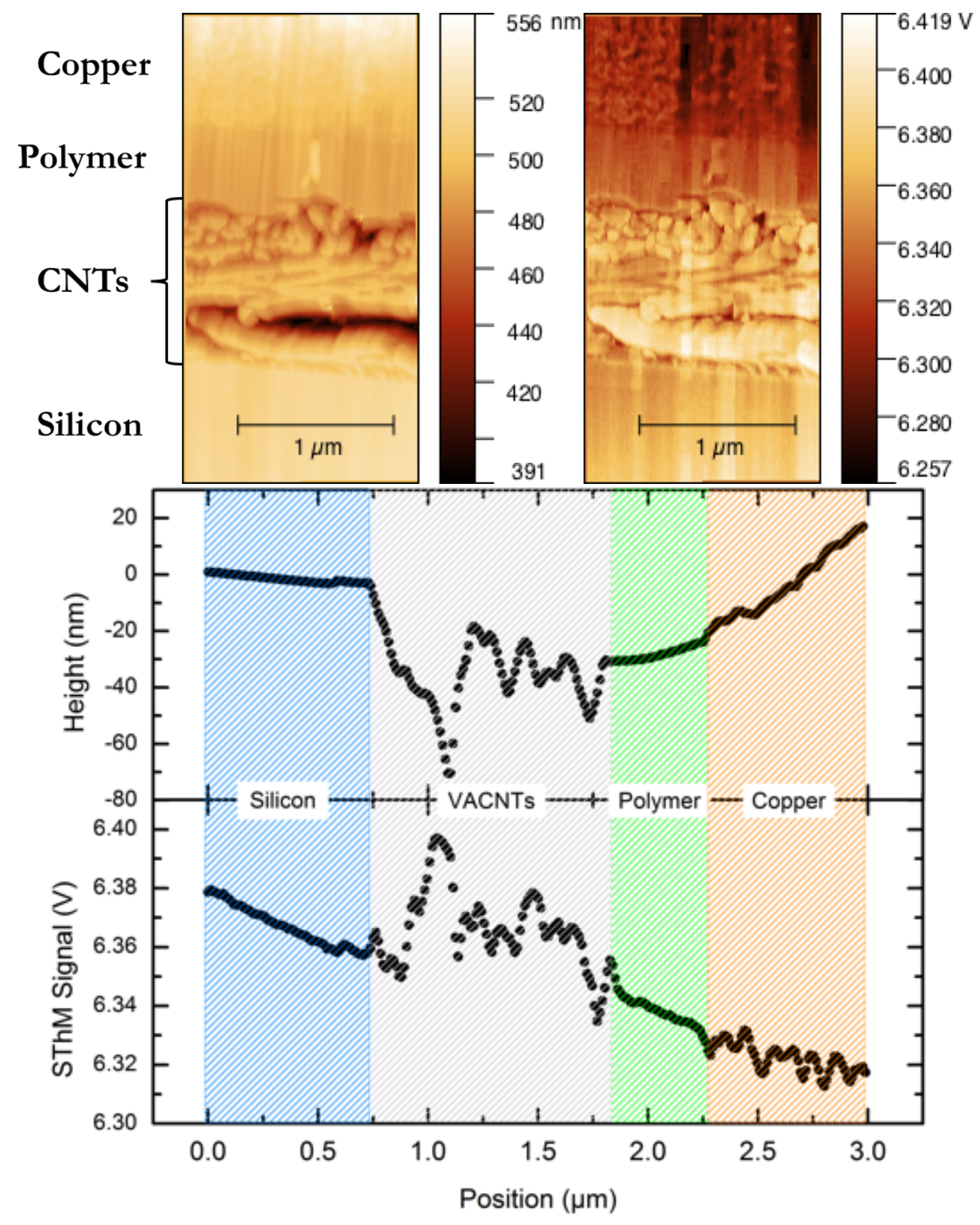

Figure 5.15: Topography (left) and SThM (right) maps and profile of the TEMlike prepared TIM sample.

\section{Studies of FIB sectioned TIM sample}

The last sample examined for nanothermal properties of full thermal interface materials was prepared by FIB polishing in a small region of its edge. To this end, the traditional copper heat sink was replaced by a thin gold layer covering the polymer (see Fig. 5.16). To fully understand its thermal behaviour, a new 
experiment was designed. Vacuum SThM measurements are performed in the FIB sectioned area while a temperature gradient is applied between both sides (Fig. 5.16 .
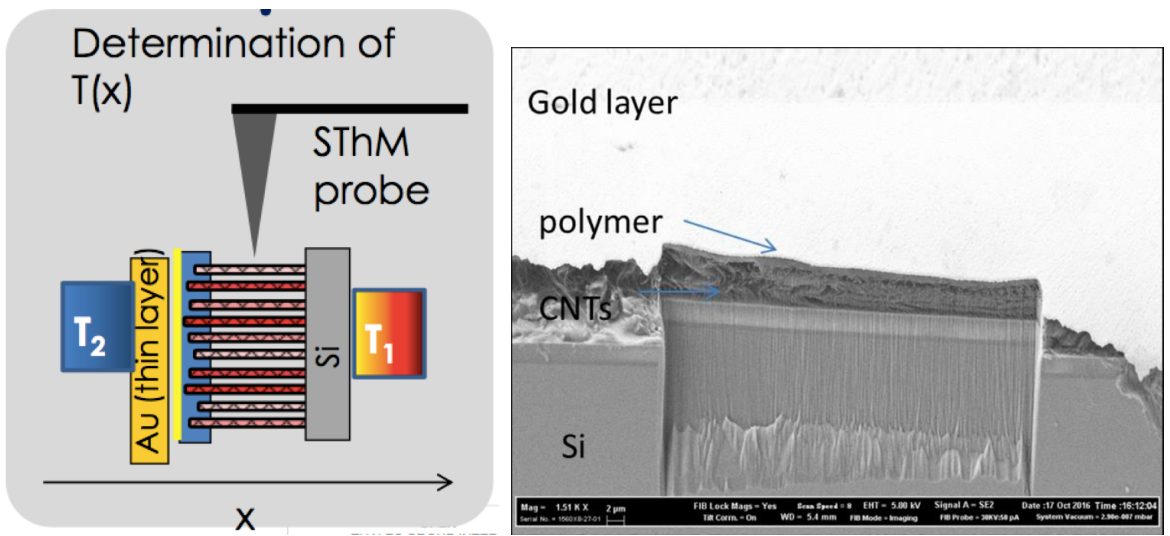

Figure 5.16: (a) Schematic of the FIB section sample experiment. While applying a temperature gradient on both sides, the temperature distribution is measured along the TIM. (b) SEM image of the FIB section.

To realise this temperature gradient, a new setup was built within the vacuum chamber as shown on Fig. 5.17. The sample is mounted on a L-shape copper support glued on a Peltier plate. This enables to keep the silicon heat sink side at a constant temperature $(\sim 300 \mathrm{~K})$. Then, to create a temperature gradient, a small $22 \mathrm{Ohm}$ resistor was used as a heat source. This resistor was thermally connected to the sample gold layer side via a gold coated copper foil. To ensure constant mechanical contact, small magnets where used on both sides of the samples, therefore maintaining mechanical contact between the foil and the sample. Then, we could approach the FIB section with the SThM (Fig. 5.17). Note that, similarly to the TEM like sample, the area of interest was small and challenging to reach with the SThM system, due to poor optical positioning setup. 


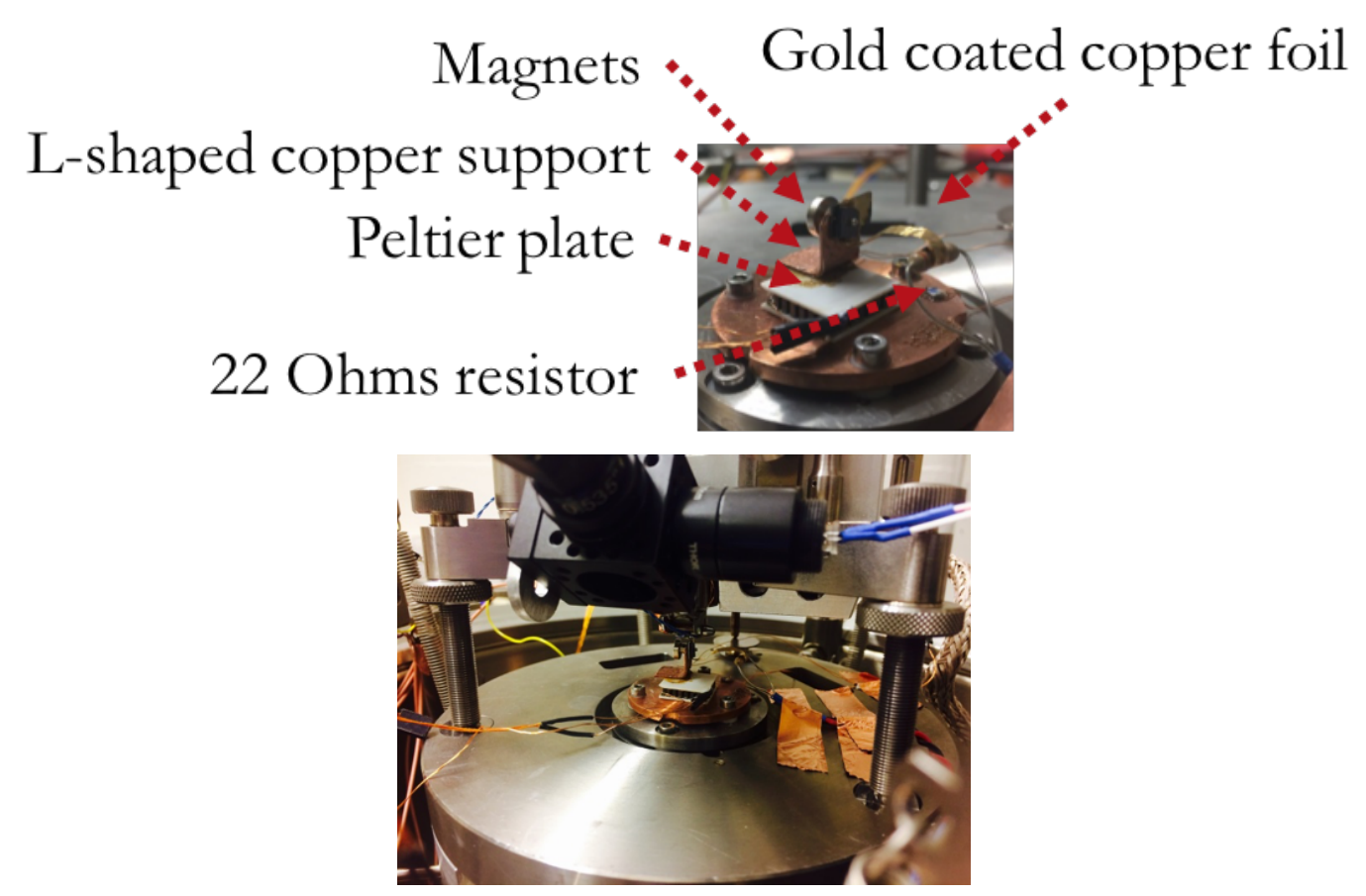

Figure 5.17: TIM temperature distribution setup and voltage bias applied on the heating resistor.

The temperature gradient was then created while applying a square voltage on the resistor (Fig. 5.18). The absolute value of the temperature rise could not be estimated. A large sample heating cycle (75s) was chosen in order to clearly observe the heating effect on the image. Results are displayed on Fig. 5.18, We note first that the FIB section leaves very rough surfaces even for the silicon material. On the top of the image, the probe is mechanical contact with the sample. We can observe the different layers in the TIM. The slow square heating is visible as creating an oscillation in the SThM signal. However, when the mechanical contact is removed, a residual variation is also observed in the SThM signal. We attributed this to heat transfer from the resistor to the probe base through the whole microscope.

Then, to distinguish the signal variation coming from the probe and the one coming from the tip, we need to subtract the non contact oscillation from the in contact signal. The resulting image is also shown on Fig. 5.18. As we observe, after subtraction, the oscillation largely disappears, meaning that most of the heating related signal variations are linked to the probe base heating. 

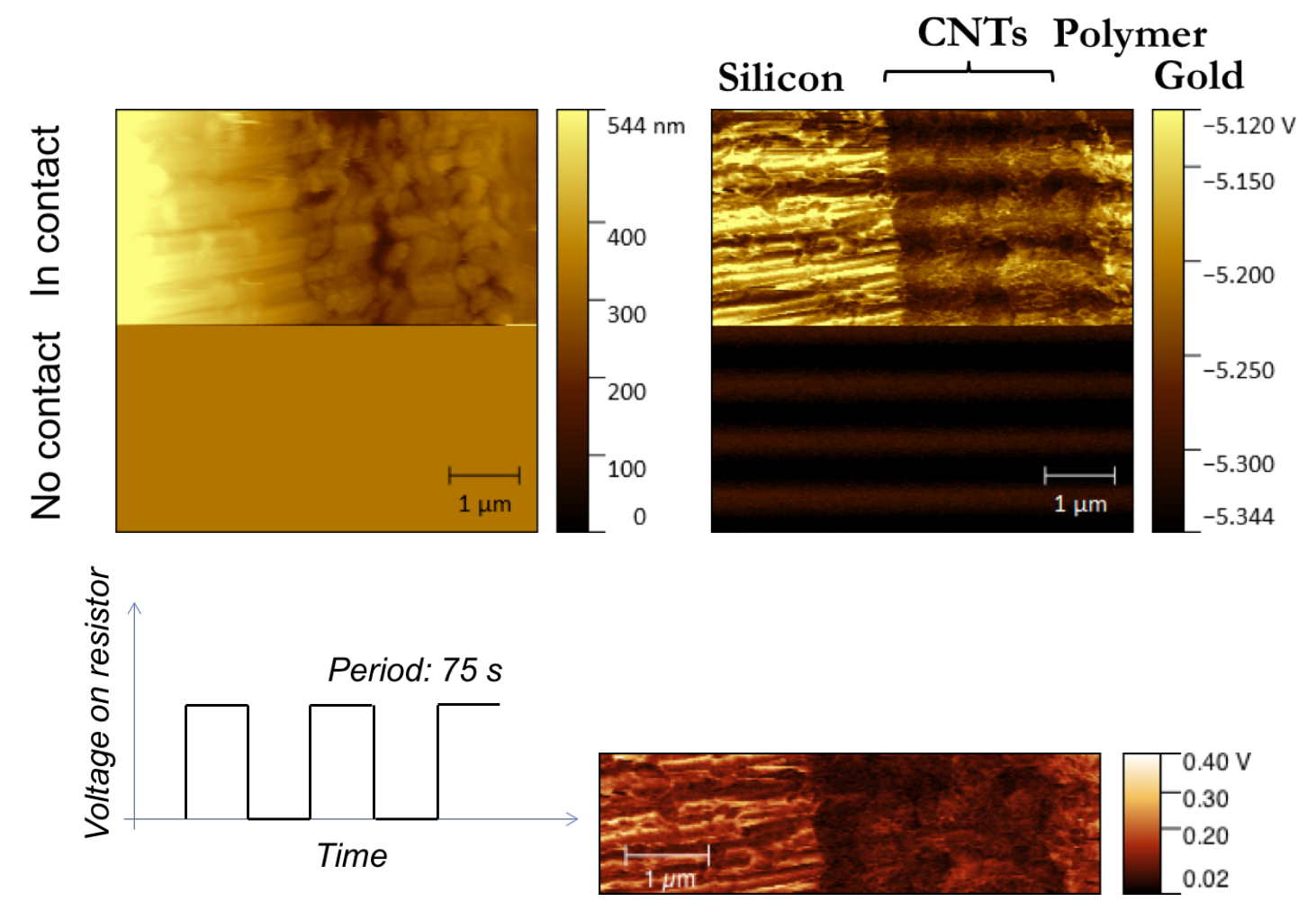

Figure 5.18: Topography (top left) and SThM (top right) temperature maps of the FIB sectioned sample when the probe is in- and out-of-contact with the sample. Schematic applied voltage on the resistor to create a temperature gradient (bottem left). Resulting SThM map (bottom right) obtained by substracting the non-contact signal from the in-contact one.

\subsubsection{Perspectives on nanothermal characterisation of TIM}

The three sample preparations presented above each present issues and challenges.

First, concerning the surface state after preparation, it appears that the perpendicular BEXP leaves very smooth surfaces but could possibly create void due to different polishing rate. The FIB cut was found to be very rough which is a major challenge for SThM measurements, although, in the case of thermometry, Menges et al. 67 introduced a powerful method which would be less affected by variation of the topography.

This brings us to measurements procedure. In the case of a TEM-like preparation, vacuum measurements would be beneficial as they would rule out parasitic air contribution to heat transfer. Although, the TEM-like procedure does not seem to offer many advantages over the FIB cut as similar system are used and 
the TEM-like ultra-thin samples are very fragile.

The last measurement procedure attempt is quite promising for TIM developments as it would allow to understand the temperature profile across the interface. The method we used was a first attempt which was proven to be ineffective. A better approach would combine either microheater located very near the FIB cut or a laser spot also heating only the area around the cut. The idea is that the heating should be preferably very local. A combination of the perpendicular BEXP and the temperature gradient approach could be promising and easily doable whereas laser heating provides a versatile, robust and easily modulated heating procedure.

We hope this foundation work will provide insights for future studies of thermal interface materials. We strongly believe that SThM can provide fundamental understanding of TIMs efficiency and powerful answers for future developments.

\subsection{Summary}

Combining a universal nano-cross-sectional tool and scanning thermal microscopy, we developed a unique method for thermal conductivity and interface thermal resistance measurements of nanostructures. Nanoscale heat transfer of materials can be investigated in 3D and provides crucial insights for new technological applications.

With an appropriate modelling approach, we were able to deduce quantitative values of thermal conductivity in thin films of materials used in semiconductor industry. This approach can be extended to different materials covering a wide range of applications.

Furthermore, we were able to quantitatively measure heat transport in anisotropic systems which is often a burden for standard techniques and demonstrated the ability to measure thermal transport in multilayered samples. Local changes of thermal resistance where successfully related to composition variations and crystalline defects. Finally, the comparison of experimental data with finite element analysis results confirms the anisotropic nature of the materials measured. 
As nanomanufacturing is becoming paramount nowadays, its thermal aspects need increasing attention. Direct applications of our results to different research areas can provide significant knowledge for advances and developments of tomorrow technologies. 


\section{Nanoscale thermal transport in low dimensional materials}

\subsection{Introduction}

Nanomaterials offer great promises of improved properties in the realisation of advanced technologies. Especially, nanostructured two dimensional (2D) and one dimensional (1D) materials exhibit interesting behaviour thanks to their low dimensionality. The exploration of heat transfer in such systems is therefore crucial for their application but requires nanoscale measurement capabilities. In this chapter, we used SThM to measure such properties of 2D and 1D systems. First, we report for the first time temperature dependent thermal conductivity of naturally occurring heterostructure franckeite and interface resistance of $\mathrm{MoS}_{2}$ and graphene. Then, we directly measure thermal conductivity in vertically aligned carbon nanotubes structures.

\subsection{Heat transport in two dimensional materials and their heterostructures}

In this section, we report the first SThM measurements of naturally occurring heterostructure franckeite as well as monolayer heterostructure of $\mathrm{MoS}_{2}$ on graphene. Heterostructures have been proposed as building blocks to tomorrow's technological developments. Stacking individual layers of different materials, as one would assemble Lego bricks together, has been proposed to form van der Waals het- 
erostructures and create on-demand new materials combining novel properties of the individual layers 180. Stacking various layered materials has already been demonstrated. Hexagonal boron nitride has been demonstrated as a high quality substrate for graphene, enhancing its electronic properties 181. For example, encapsulated graphene in hexagonal boron nitride shows a room temperature ballistic electronic transport with micrometer scale 182 . Recently, Tielrooil et al. 183 demonstrated an increased electron-phonon coupling between graphene encased in boron nitride 182 suggesting an efficient heat dissipation of such devices.

Using our unique low temperature SThM setup we could measure thermal conductivity and interface resistance of natural heterostructure material franckeite on silicon oxide, which, for the best of our knowledge, has not been reported so far. Then, with the same system, we explored manually stacked single layer $\mathrm{MoS}_{2}$ on monolayer graphene, resting on silicon oxide. With similar models as presented above, thermal properties can be deduced for temperature between 150 and 300 K. The samples were prepared by Dr. Aday Molina-Mendoza and Assoc. Prof. Thomas Mueller from the Institute of Photonics, TU Wien.

\subsubsection{Franckeite}

In the quest to find interesting properties in the wide variety of layered materials available, natural 2D materials heterostructures are appealing. Natural heterostructures are layered materials whose atomic structure changes at each layer up to form a unit cell of several layers. The advantage of such a material is evident from a fabrication point of view. Having a naturally occurring heterostructure could then potentially solve the long and costly fabrication process of non-natural heterostructures while at the same time improving the sample cleanness and decrease the randomness introduced by the fabrication. It could also have improved interfaces properties between individual layers.

Franckeite has been proven attractive for its narrow bandgap and p-type doping and, combined with $\mathrm{MoS}_{2}$, successfully created a mid-infrared detector 184. The 
Franckeite atomic structure contains stacked alternating $\mathrm{SnS}_{2}$ like and PdS-like layers 185. Some variability in the stoichiometry was reported 184 but a general structure can be outlined. A unit cell of Franckeite is composed of stacked pseudohexagonal $\mathrm{H}$ and pseudotetragonal Q layers, see Fig. 6.1. The $\mathrm{H}$ layer contains disulfide compounds $\mathrm{NX}_{2}$ where $\mathrm{N}$ can be either $\mathrm{Sn}$ or $\mathrm{Fe}$ and $\mathrm{X}=\mathrm{S}$ while the $\mathrm{Q}$ layer consists of $\mathrm{MX}$ where $\mathrm{M}$ is $\mathrm{Pb}, \mathrm{Sn}$ or $\mathrm{Sb}$ and $\mathrm{X}=\mathrm{S}$. It is worth noting that $\mathrm{M}$ or $\mathrm{N}$ atoms are varying within a single layer. For example, a single Q layer will have $\mathrm{Pb}, \mathrm{Sn}$ and $\mathrm{Sb}$ atoms and will not consists only of one atom type 185 .

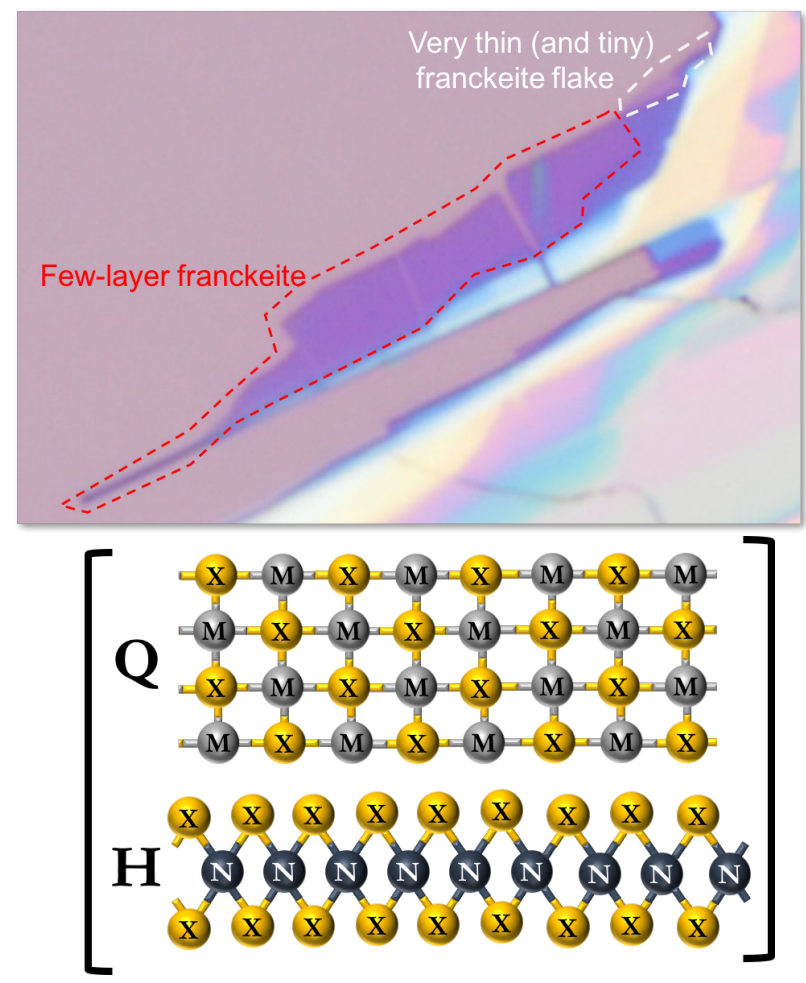

Figure 6.1: Optical microscope image of the Franckeite flake with various numbers of layer and franckeite unit cell structure where $\mathrm{M}$ can be $\mathrm{Pb}$, $\mathrm{Sn}$ or $\mathrm{Sb}, \mathrm{N}$ is either Sn or Fe and $\mathrm{X}$ is $\mathrm{S}$.

While franckeite electrical and optical properties have been reported [186], its thermal properties have to be investigated to realise fully functioning devices. Due to the microscale of exfoliated flakes, standard measurement techniques are not the optimum choice to understand nanoscale thermal transport within franckeite nanostructures. It is therefore natural to turn to Scanning Thermal Microscope to measure franckeite nanoscale heat transport.

Using the vacuum SThM setup with a doped Si probe described in chapter 
3. we mapped franckeite thermal response (see Fig.6.2). The area was chosen using optical microscopy as it was presenting various thicknesses estimated to be from 3 to 39 layers. Optical microscope image is displayed on Fig. 6.1. The thermal resistance can be seen to increase with increasing franckeite thickness. Such behaviour is expected when the layer thermal conductivity is smaller than the one of the substrate.
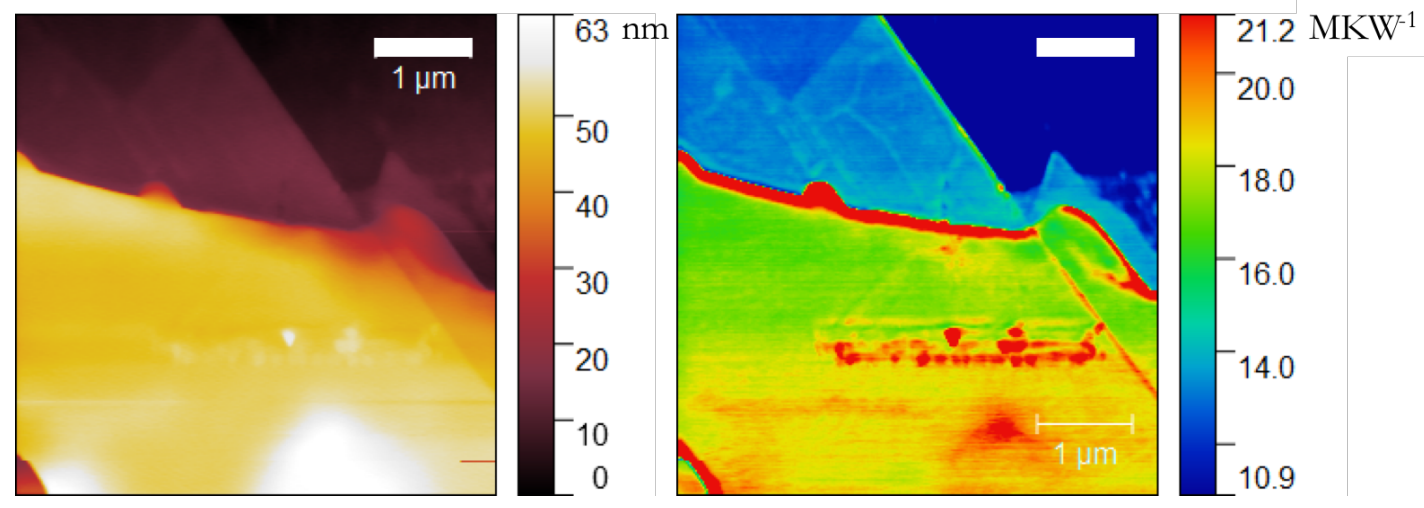

Figure 6.2: Topography (left) and SThM (right) maps of an exfoliated franckeite flake. Several thickness can be observed with different thermal response. As the franckeite thickness increases, its thermal resistance increases as well. This behaviour is the signature of a lower thermal conductivity material than the silicon oxide substrate.

To get a quantitative estimate of the thermal conductivity of franckeite and the thermal interface resistance between the flake and the silicon oxide, we turn to similar spreading resistance models as used in previous chapters. We model the flake as a uniform layer on a substrate similarly to the oxide layers presented above and using Eq. 4.4. The thermal contact radius $(40 \mathrm{~nm})$ between the probe and the sample is estimated from SEM imaging of the tip and inspection of the thermal images (see Appendices).

By using Eq. 4.4, we here ignore the anisotropy of the franckeite crystal structure and obtain therefore effective quantities. As already observed in Fig. 6.2, a lower thermal resistance is obtained from the franckeite as compared to the silicon oxide substrate. This behaviour is the signature of a low effective thermal conductivity material and the diffusive assumption is realistic in this material measured at these length scales. Indeed, considering the relatively big contact radius and 
the structure of the franckeite, the phonon MFP will be much smaller than the heat source contact. Due to the high anisotropy and high composition variations, phonon scattering will be important and lead to a very small phonon MFP.

Fig. 6.3 shows the franckeite thermal resistance as a function of the layer thickness for various sample temperatures. We fitted the experimental data with the spreading resistance model as represented on the graph. To quantify the thermal conductivity and interface resistance values obtained, we need to analyse the tip and tip-sample resistances.

In the equation $R_{X}=R_{\text {tip }}+R_{\text {int }}+R_{s p r}$, we tried two approaches for the unknown $R_{t i p}+R_{\text {int }}$. In first approach, we estimate it from the thermal resistance obtained on the silicon oxide substrate. As for the substrate $R_{s p r}=\frac{1}{4 k_{S i O x} a}$ is known at various temperature, we can deduce $R_{t i p}+R_{\text {int }}$, obtaining resistances between 3 and $5 \times 10^{6} \mathrm{KW}^{-1}$. These values therefore include the contribution of the conical tip and the interface between the silicon oxide covered oxide tip and silicon oxide substrate. Assuming that $R_{t i p}$ is the same when contacting the franckeite is reasonable but $R_{\text {int }}$ is likely to be different due to difference surface roughness and mismatch. 


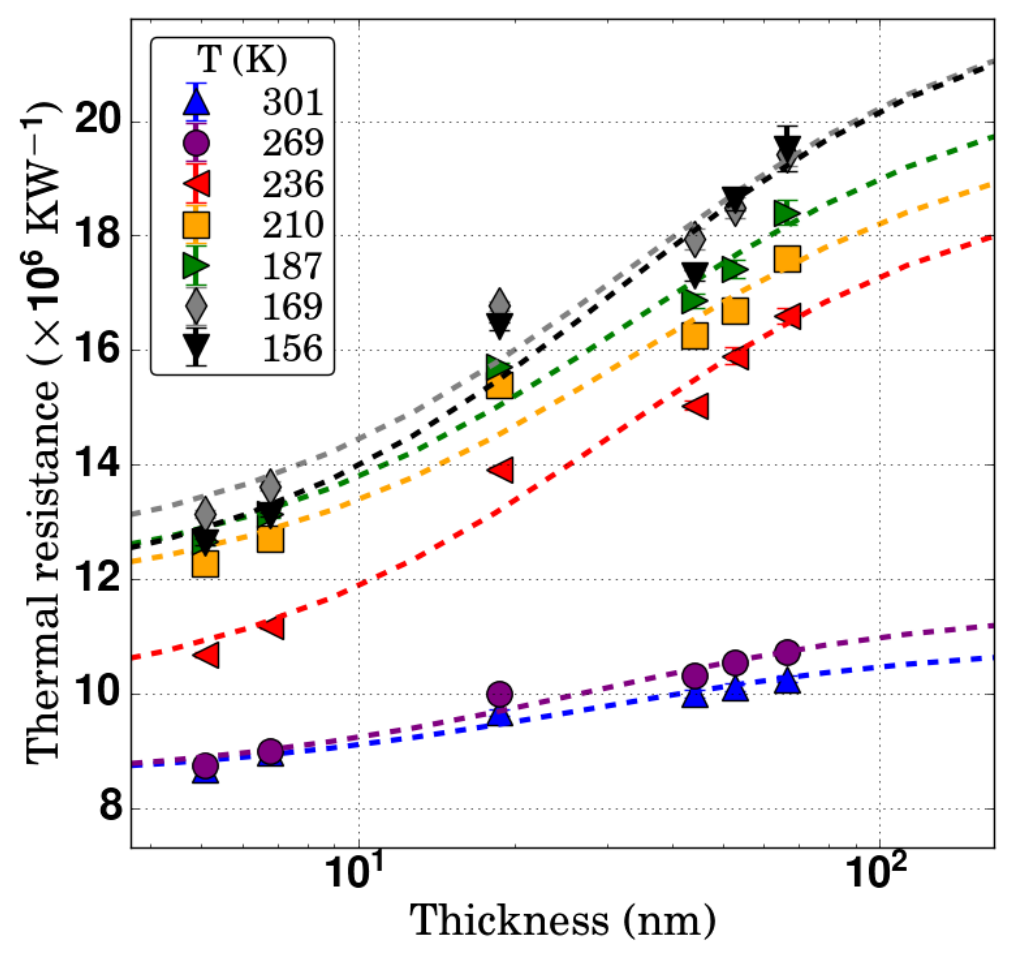

Figure 6.3: Thermal resistance received from the SThM meaurements as a function of franckeite thickness for temperatures from 156 to $301 \mathrm{~K}$. The experimental data is plotted with symbols while the spreading resistance model of Eq. 4.4 is shown in dashed lines.

In a general approach, we left $R_{\text {tip }}+R_{\text {int }}$ as a fitting parameter and obtained values slightly higher between 4 and $5 \times 10^{6} \mathrm{KW}^{-1}$. Having higher values is not counter intuitive as the mismatch is expected to be more important between the silicon oxide covered tip and the franckeite than between the tip and silicon oxide substrate. However, the data obtained confirm the accuracy of the fitting method.

For the thermal conductivity $k$ and interface resistance $r_{i n t}$, we applied both approaches for $R_{t i p}+R_{\text {int }}$ in the fitting function applied the experimental data. Both methods produced similar values within $10 \%$ for $k$ and $r_{\text {int }}$. These results obtained from the general approach are shown in Fig. 6.4. The thermal conductivity is decreasing with decreasing temperature while $r_{i n t}$ increases. A decreasing conductivity is also found in supported graphene 187, encased graphene and ultrathin graphite 188.

The interface resistance increase with decreasing temperature can be compared 
to results obtain on artificial heterostructures. Graphene on hexagonal boron nitride 189 and graphene on $\mathrm{MoS}_{2}[190$ interface resistances were reported to increase by almost an order of magnitude in our range of temperature, similarly to the results obtained between franckeite and silicon oxide.
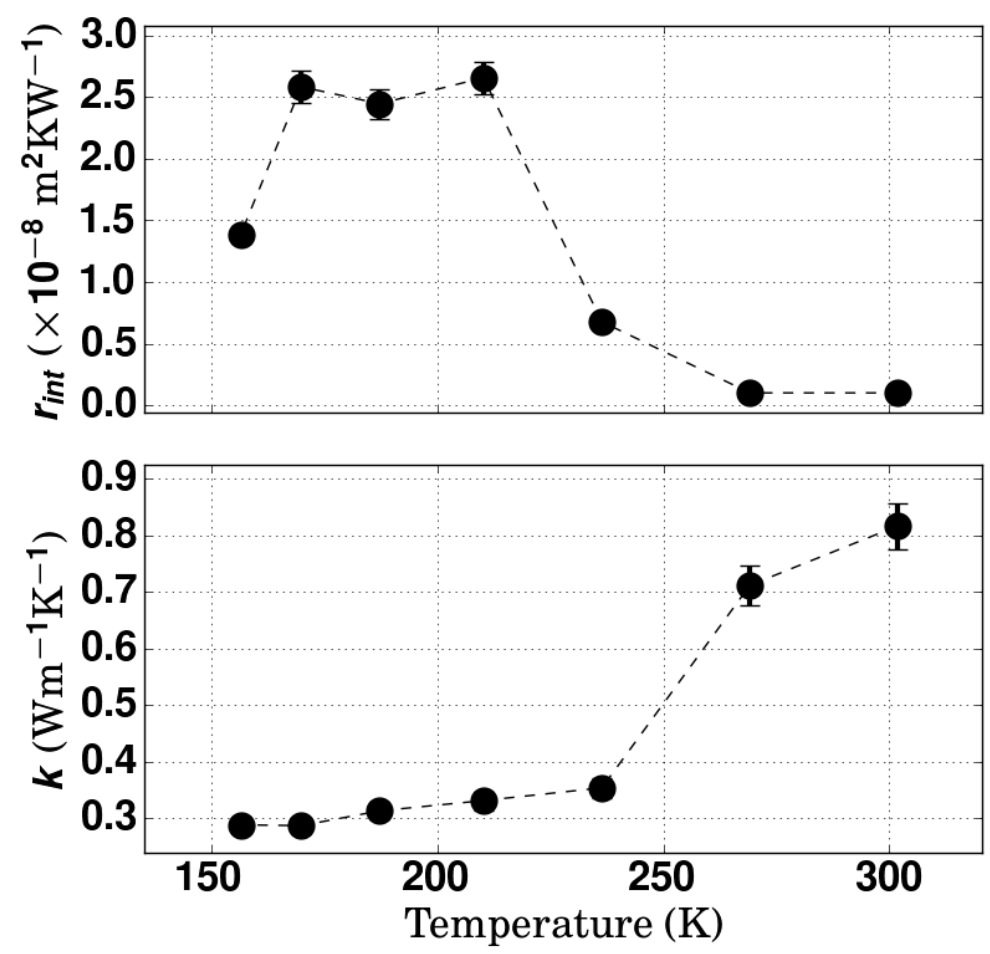

Figure 6.4: Interface thermal resistance $r_{i n t}$ and thermal conductivity of franckeite as a function of temperature as obtained from the fitting of Eq. 4.4 on the data shown in Fig. 6.3

These measurements report for the first time thermal transport properties of franckeite on silicon oxide. We hope that these results will provide fruitful insights to fully exploit franckeite potential in device applications.

\subsubsection{Heterostructure of $\mathrm{MoS}_{2}$ on graphene}

In the previous section, we studied a naturally occuring hetersostructure and now we proceed to a fabricated one consisting of a monolayer graphene and monolayer $\mathrm{MoS}_{2}$ on silicon oxide. The monolayer was deposited on top of the graphene flake by a pick-and-place method that allows to pick-up an atomically-thin flake from a substrate with the subsequent transfer on another substrate[191. Graphene and $\mathrm{MoS}_{2}$ where exfoliated by standard scotch tape method on a silicon oxide (280 nm) 
substrate and the atomically-thin flakes were identified by optical microscopy. The $\mathrm{MoS}_{2}$ monolayer was then picked-up and transferred on top of the graphene flake, ensuring a clean interface between the two materials (see Fig. 6.5).

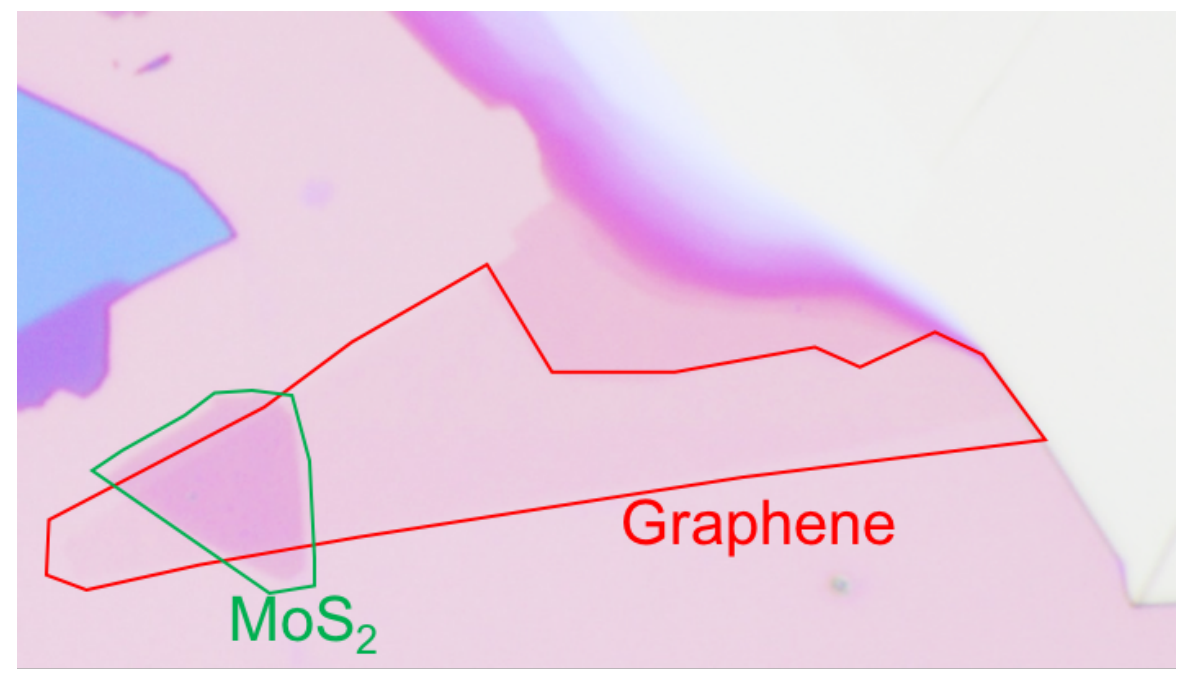

Figure 6.5: Optical microscope image of the $\mathrm{MoS}_{2}$ on graphene heterostructure.

An example of topography and SThM image is shown in Fig. 6.6. The image shows areas of graphene on $\mathrm{SiO}_{2}, \mathrm{MoS}_{2}$ on $\mathrm{SiO}_{2}$ and $\mathrm{MoS}_{2}$ on top of graphene on $\mathrm{SiO}_{2}$. The SThM image shows the thermal resistance response of each area. As expected, graphene, with its high conductance, reduces the thermal resistance compared to the substrate. Interestingly, for $\mathrm{MoS}_{2}$ whose thermal conductivity on silicon oxide has been reported around $55 \mathrm{Wm}^{-1} \mathrm{~K}^{-1}[192,193]$, the thermal resistance obtained is higher than the oxide substrate. When scanning the heterostructure, the probe is contacting the $\mathrm{MoS}_{2}$ resting on the graphene. Here we can compare all resistances of areas of $\mathrm{SiO}_{2}$, graphene on $\mathrm{SiO}_{2}, \mathrm{MoS}_{2}$ on $\mathrm{SiO}_{2}$ and $\mathrm{MoS}_{2}$ on top of graphene on $\mathrm{SiO}_{2}$ 


\section{Topography}

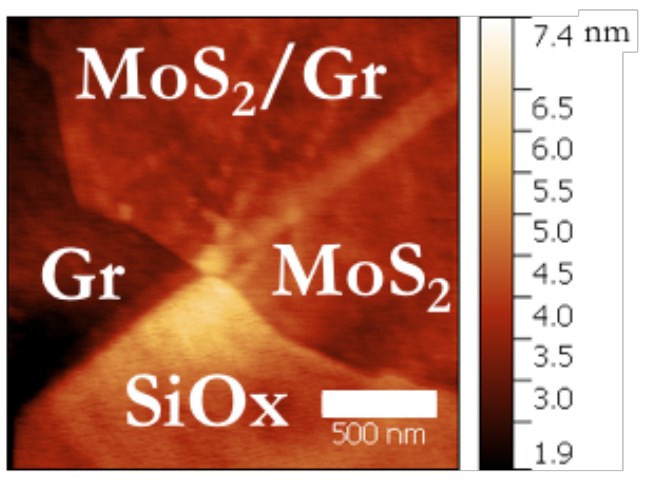

SThM

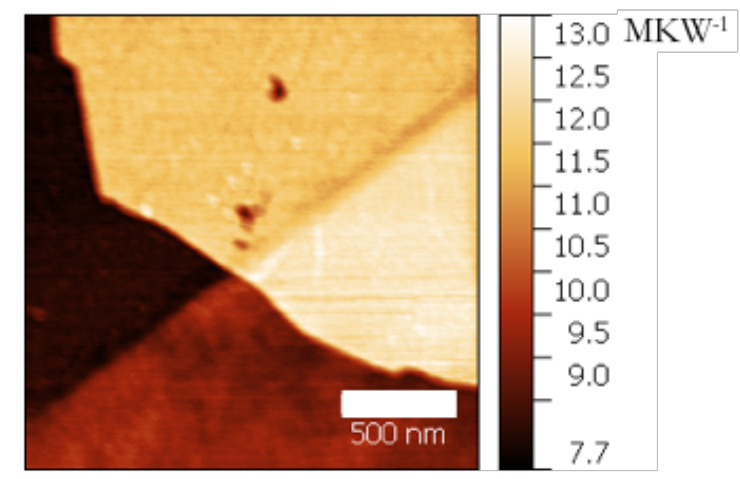

Figure 6.6: Topography and SThM images of $\mathrm{MoS}_{2}$ on graphene heterostructure obtained at $220 \mathrm{~K}$. The graphene has a lower resistance than the $\mathrm{MoS}_{2}$ and it appears that graphene lower the resistance of the $\mathrm{MoS}_{2}$ when it is below. Scale bar is $500 \mathrm{~nm}$.

We discuss first the interesting contrast obtained on the monolayer $\mathrm{MoS}_{2}$ resting on oxide. As we mentioned the layer thermal conductivity is higher than that of the substrate. Therefore one would expect from the spreading resistance model to obtain a lower response compared to the oxide response. Furthermore, the resistance measured on graphene is lower than that of silicon oxide. Thus, clearly, the layer thermal conductivity doesn't play a major role in the thermal resistance sensed by the SThM tip. When looking at values reported in the literature for interface resistance between $\mathrm{MoS}_{2}$ or graphene and silicon oxide, we find a potential explanation to the contrast observed. The interface $\mathrm{MoS}_{2}$-oxide has values reported between $4 \times 10^{-8}$ to $2.27 \times 10^{-6} \mathrm{~m}^{2} \mathrm{KW}^{-1}$ 192, 194, 195. In contrast, the interface resistance between graphene and silicon oxide is lower. Values from $5.6 \times 10^{-9}$ to $2 \times 10^{-8} \mathrm{~m}^{2} \mathrm{KW}^{-1}$ have been reported 187, 196, 197.

Fig. 6.7 shows the thermal resistance as a function of sample temperature for the four different areas and the sample. For all temperatures, the same contrast as observed on Fig. 6.6 is preserved. A general increasing trend is received with decreasing temperature. This links directly with the reducing thermal conductivity of the silicon oxide substrate as discussed in the previous chapter. 


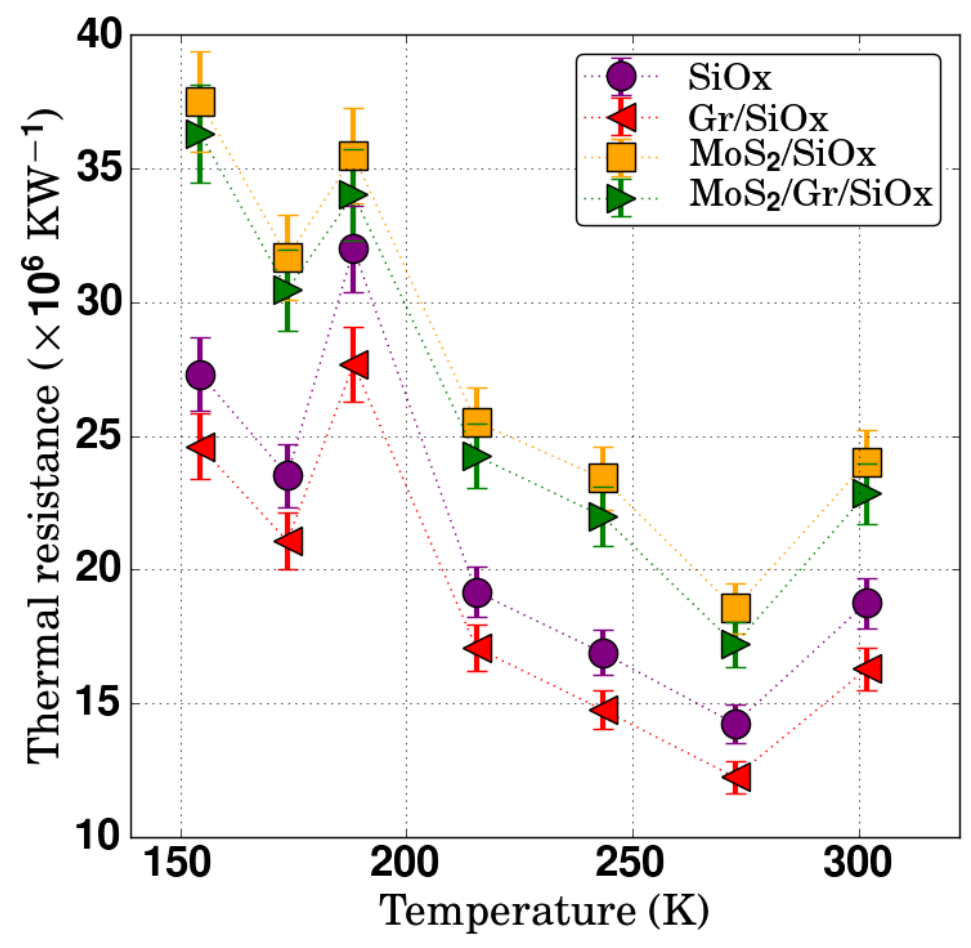

Figure 6.7: Thermal resistance as a function of sample temperature for the different areas of the sample. A general increase of the thermal resistance is observed for all regions.

If we assume that the thermal resistance is dominated by interface effects, we have the following relations on the different areas for the thermal resistance between the heater and the sample heat bath:

Oxide:

$R_{\text {tip }}+R_{\text {int }_{o x-o x}}+R_{\text {spr }}$

Graphene on oxide:

$R_{\text {tip }}+R_{\text {int }_{o x-g r}}+R_{g r}+R_{\text {int }_{o x-g r}}+R_{\text {sprox }}$

$\mathrm{MoS}_{2}$ on oxide:

$R_{\text {tip }}+R_{\text {int }_{o x-M o S_{2}}}+R_{M o S_{2}}+R_{\text {int }_{o x-M o S_{2}}}+R_{\text {sprox }}$

$\mathrm{MoS}_{2}$ on graphene on oxide:

$$
R_{\text {tip }}+R_{\text {int }_{o x-M o S_{2}}}+R_{M o S_{2}}+R_{\text {int }_{M o S_{2}-G r}}+R_{g r}+R_{\text {sprox }_{o x}}
$$


The conical tip resistance $R_{t i p}$ is unknown but the spreading resistance into the silicon oxide is defined as $R_{s p r_{o x}}=1 / 4 k_{S i O x} a$ where $a$ is the contact radius (40 nm from SEM imaging and image inspection, see Appendices) and $k_{S i O X}$ the temperature dependent oxide thermal conductivity. We adopt a macroscopic expression for interface resistances and define $R_{\text {int }}$ as the interface resistance $r_{i n t}$ (expressed in $\mathrm{m}^{2} \mathrm{KW}^{-1}$ ) normalised to the contact area: $R_{\text {int }}=r_{i n t} / \pi a^{2}$. Finally, based on the observation described above that layer thermal conductivity isn't affecting strongly the measured resistance, we assume that for graphene and $\mathrm{MoS}_{2}$, $R_{\text {layer }} \approx 0$. This assumption is similar to work performed by Chen et al. [196]. However, their work is based on microscopic heaters while in our case such an assumption has limitations.

If we assume some values for $R_{t i p}$, then we can deduce $r_{i n t_{g r-o x}}, r_{\text {int }_{M o S_{2}-o x}}$ and ultimately $r_{\text {int }_{M S_{2}-g r}}$. To estimate $R_{t i p}$, we can remove the contribution of the spreading resistance from the measurements performed on the oxide substrate. However, we still need to estimate $R_{\text {int } t_{o x-o x}}$, the interface between the oxide covered tip and the oxide. Such resistance is unknown and is one of the main challenges of SThM measurements. We will therefore need to renormalise the $R_{\text {intox-ox }}$ value such that it would give similar values for $r_{i n t_{g r-o x}}$ found in the literature, obtaining $R_{\text {int }_{\text {ox }-o x}}=7.9 \times 10^{6} \mathrm{KW}^{-1}$.

Using the model presented here, we can extract interface thermal resistance for $\mathrm{MoS}_{2}$ and graphene to silicon oxide as well as $\mathrm{MoS}_{2}$ to graphene. Results are displayed on Fig. 6.8. As in the literature, the $\mathrm{MoS}_{2}$ to oxide interface has higher thermal resistance than the graphene to oxide interface. 


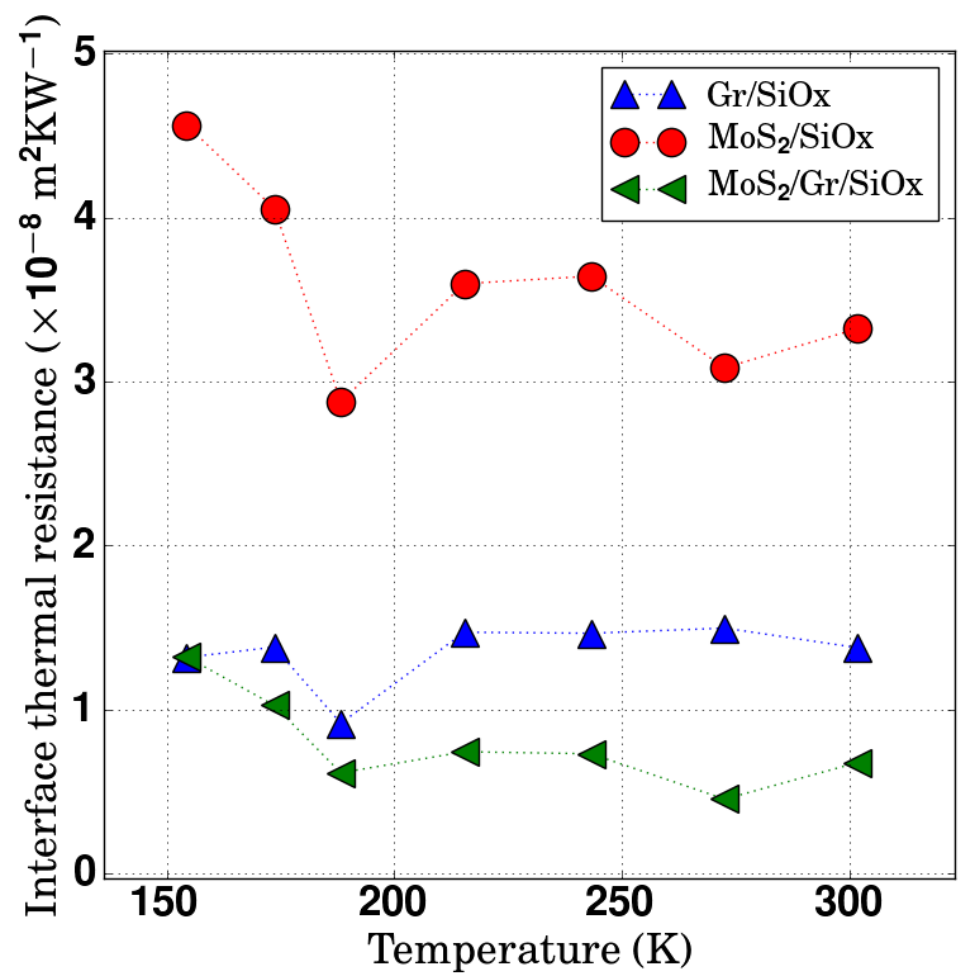

Figure 6.8: Measured interface thermal resistance as a function of sample temperature for various interfaces.

We reported here SThM measurements of heterostructure composed of $\mathrm{MoS}_{2}$ on graphene at temperatures from $150 \mathrm{~K}$ to $300 \mathrm{~K}$. Thermal resistances were extracted for various areas corresponding to different materials and interfaces. A general increase of the thermal resistance is received corresponding to the change of the substrate properties. Furthermore, with a realistic heat transport model, we could obtain interface thermal resistance values for $\mathrm{MoS}_{2}$ and graphene to oxide as well as for the $\mathrm{MoS}_{2}$-graphene interface. Considering the simplicity and assumptions of our model, the values obtained are comparable to literature values. 


\subsection{Temperature dependent thermal resistance of length varying vertically aligned carbon nan- otubes}

Carbon nanotubes (CNTs) forests are being studied for high thermally conductive thermal interface applications $179,198,200$. Thanks to their high thermal conductivity, they could enhance heat dissipation and serve in thermal management. To design better thermal interface materials (TIM), two aspects need to be addressed: nanoscale thermal properties and thermal properties of buried CNT-sample interface. In this section, we address both questions and report measurements using the BEXP-SThM method introduced in chapter 5.

Vertically aligned nanotubes grown on a silicon substrate were polished at shallow angle using BEXP. Thanks to this procedure softness, the alignment and structure was preserved and an array of length increasing nanotubes was created. After assessing the sample structure with SEM imaging, we measure the thermal resistance using a sharp SThM probe in vacuum. From the resistance variation observed with increasing nanotubes height, we can deduce thermal conductivities both along and perpendicularly to the nanotubes axis. Finally, we probe the temperature dependence of the thermal transport in these structures by cooling the sample to $200 \mathrm{~K}$. A sharp thermal resistance increase is observed linked to the reduction of CNT thermal conductivity.

This work reports for the first time a unique sample preparation opening new study perspectives of high aspect ratio 1D materials nanostructures. Our results provide useful information for nanotubes array applications and design of thermal interface materials. Samples investigated in this section were provided by Thales collaborators, in particular Dr. Odile Bezencenet. Also, SEM imaging was performed by Dr. Kunal Lulla, in Lancaster. 


\subsubsection{Sample preparation and description}

A forest of vertically aligned carbon nanotubes was grown on a silicon substrate. The average nanotube diameter is $10 \mathrm{~nm}$. The sample was then polished using BEXP 159]. Fig. 6.9 shows SEM images of the cross-sectioned nanotubes that reveal aligned nanotubes. A major observation is that the nanotubes keep their arrangement and verticality and are found not to be affected by the BEXP process. This opens a new range of possibilities of this polishing technique for new potential studies and applications. For example, vertically grown nanowires could be studied similarly. Also, a useful result we can observe is that the BEXP process created an array of CNTs with increasing height. This will be exploited hereafter to obtain the thermal conductivity of the nanotubes forest using an approach described in chapters 4 and 5 .

However, as observed on Fig 6.9 $\mathrm{b}$ and on SEM images of Fig 6.10, the CNTs form bundles consisting of several individual aggregated nanotubes. The average bundle diameter was estimated to be around $24 \mathrm{~nm}$. This would correspond to approximately 3 nanotubes bundled together. In fact, it appears that this bundling effect almost begins at the seed layer. Also, from SEM imaging and standard wetting experiments performed by the sample provider, the CNT coverage obtained is around $28 \pm 2 \%$. 


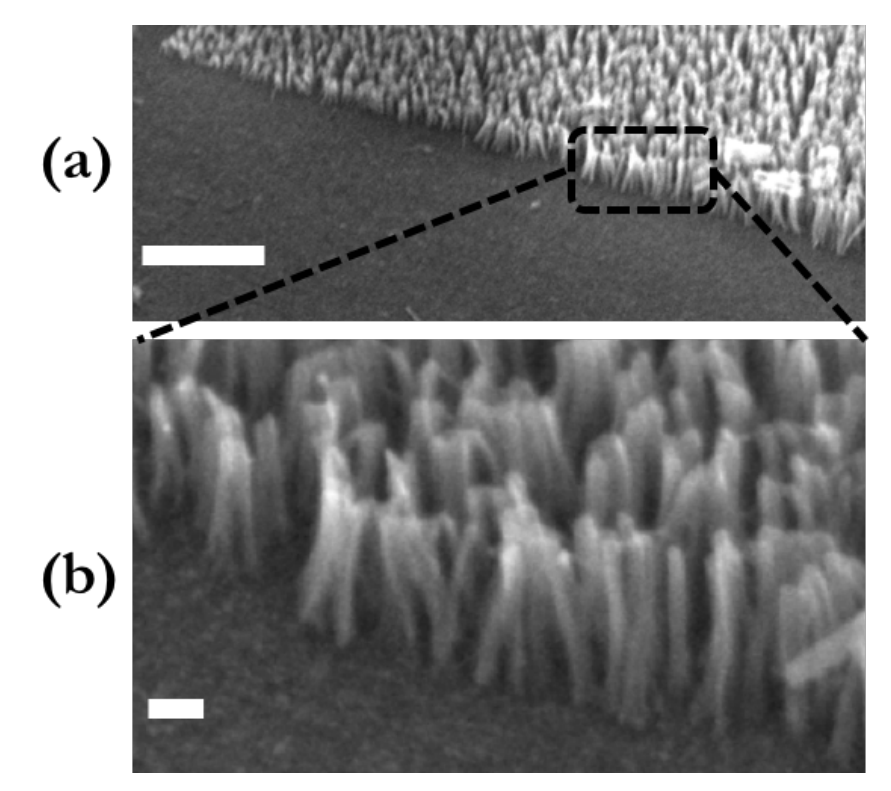

Figure 6.9: (a) SEM imaging of the nanotubes bundles with increasing height, scale bar is $1 \mu m$. (b) Magnified SEM image of the CNT bundles. Scale bar is 100 nm.

SThM was used in vacuum with a sharp doped silicon probe to map the topography and thermal conductance of these bundles (Figs. 6.10 b-c). The topography image shows a distribution of bundle sizes as expected from the SEM images. Furthermore, a dip is obtained between the bundles highlighting the sharpness of the probe used. Higher SThM signal is received between the bundles. This is attributed to increased contact area between the tip and the sample. As individual bundles are resolved both in topography and SThM maps, we deduce that the tip-sample contact diameter is smaller than the bundle diameter. 
(a)

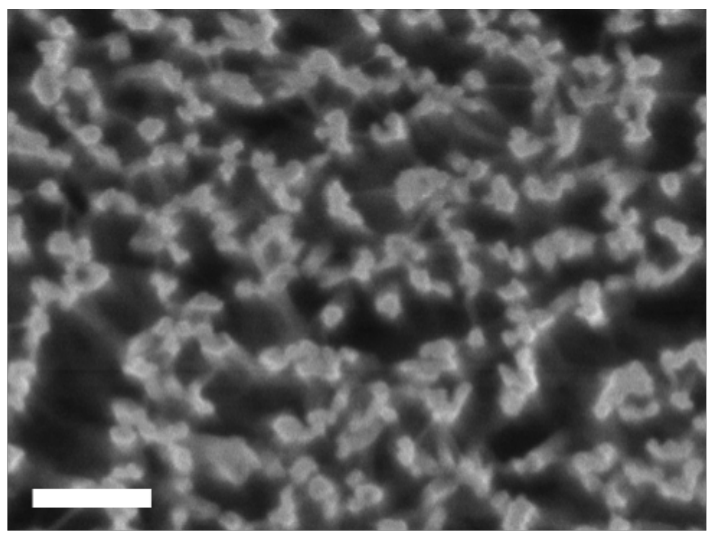

(b)

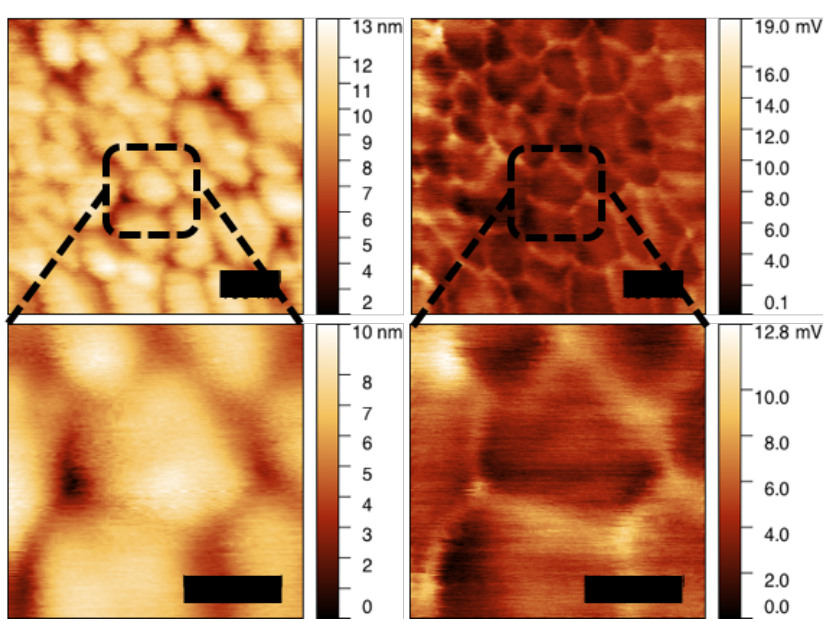

Figure 6.10: (a) Top view SEM image showing around $28 \pm 2 \%$ coverage of CNTs. Scale bar is $200 \mathrm{~nm}$. (b)-(c) Topography (left) and SThM (right) maps of the nanotubes bundles. Scale bars are $100 \mathrm{~nm}$ (b) and $50 \mathrm{~nm}$ (c).

\subsubsection{Thermal resistance measurement and models}

The voltage measured in the experiment can be linked to an absolute temperature via a calibration of the probe resistance. We have $V=\alpha \Delta T=\alpha\left(T_{h}-T_{0}\right)$. As we measure the probe thermal resistance $\left(R_{p} \approx 4.5 \pm 0.1 \times 10^{4} \mathrm{KW}^{-1}\right.$, see chapter 3) and the probe base and sample temperatures $\left(T_{B}=T_{s}=T_{0}=300 \mathrm{~K}\right)$, we can extract the total thermal resistance measured $R_{x}$ as we explained in chap. 3. Fig. 6.11a shows a plot of this resistance as a function of the bundle height as measured by the SThM. We located the x-axis origin at the beginning of the vertically aligned CNTs layer for convenience. Hence, negative heights correspond to materials below the nanotubes.

On Figs. 6.11b-c, SThM and UFM maps of this sample are shown. The 
UFM response describes the sample stiffness. As it can be expected, the silicon substrate is stiffer than the nanotubes bundles as bending stiffness is low due to their flexibility. On the SThM map, the silicon substrate appears less thermally resistive. There are two main explanations for this observation. First, it is likely that the thermal contact between the probe and the silicon is better than the one between the probe and the bundles. Second, the bundles, or any layer, act as an extra resistance between the sensor and the sample heat sink and as such appear more resistive. On Fig. 6.11a, the seed layer between the silicon and the nanotubes can be also resolved.

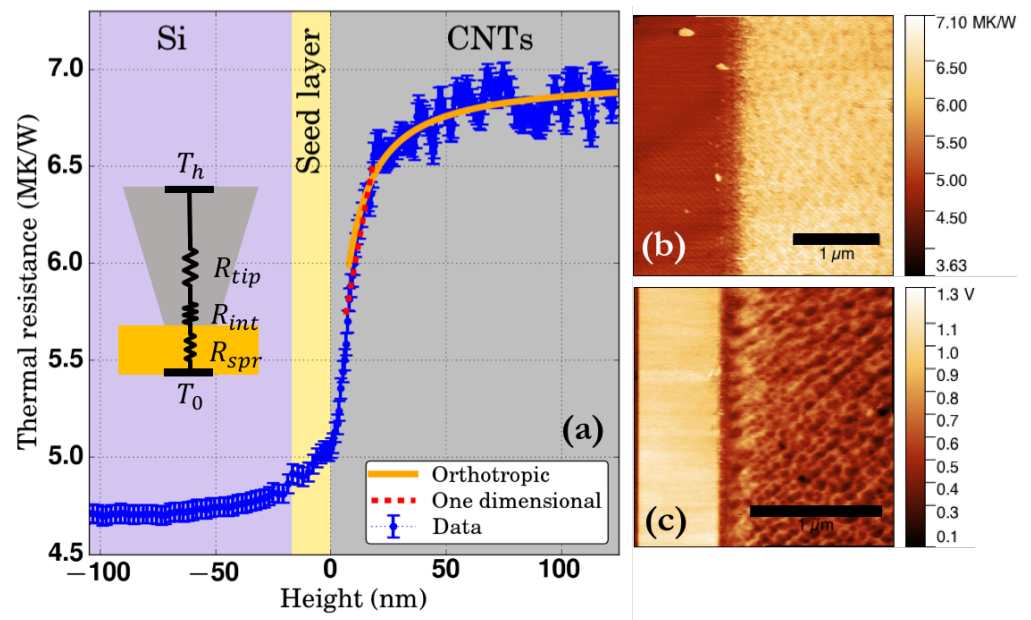

Figure 6.11: (a) Thermal resistance measured as a function of nanotubes height and fitting models applied. Inset shows the thermal resistances included between the sensor and the heat sink. SThM (b) and UFM (c) maps of the silicon-nanotubes interface, scale bars are $1 \mu \mathrm{m}$.

The thermal resistance measured is composed of several resistances in series: first, the resistance of the tip itself created mostly by the constriction in the conical tip; then, as in any thermal contact, a material dependent interface resistance between the tip apex and the sample; and finally, the spreading resistance inside the sample. In summary, we have:

$$
R_{X}=R_{\text {tip }}+R_{\text {int }}+R_{\text {spr }}
$$

$R_{\text {tip }}$ can be assumed to be constant for all the sample but $R_{\text {int }}$ is likely to change drastically between the tip-Si substrate and the tip-nanotubes contacts as 
we discussed from the SThM and UFM maps on Figs. 6.11b-c. However, we can assume that while scanning within the same layer, for example, the nanotubes only, the interface thermal resistance $R_{\text {int }}$ remains constant. So, for the same material, the sum $R_{\text {tip }}+R_{\text {int }}$ can be reasonably considered constant.

To extract thermal conductivity values of the cross-sectioned nanotubes, two models can be applied to $R_{s p r}$. These models are all diffusive and don't consider any ballistic transport that could occur in this system. We will discuss ballistic transport contribution in the next section when low temperature transport is investigated. In the two diffusive models we develop here, we estimate that phonon scattering between the nanotubes forming each bundle greatly reduce the mean free path 201, 202]. Also, as the probe-bundle contact area is on a similar order of magnitude, a ballistic constriction resistance is not expected.

The first model considers the sample as an array of vertically aligned cylinders and assumes one dimensional (1D) transport along these cylinders. The thermal resistance can be written as a function of the bundle height $L_{\text {bundle }}$ :

$$
R_{\text {bundle }}=\frac{L_{\text {bundle }}}{k_{\text {bundle }} A}
$$

where $A$ is the cross-sectionnal area of the bundle and its thermal conductivity. Cross-sectional area is ill-defined in the case of a single nanotube and thus for nanotubes bundles 203]. For a single tube, it can be defined as $A=\pi d \delta$ where $d$ is the tube diameter and $\delta$ the layer separation of graphite. However, as in our case we are dealing with bundles, we consider the bundles as isotropic materials and as such we find a lower bound of the thermal conductivity 65 .

As we see from the data in Fig. 6.11a, the thermal resistance measured saturates around $40 \mathrm{~nm}$ enlightening the fact that heat spreading also occurs perpendicularly to the nanotubes axis. Then to model our samples as an array of cylinders, we will use only the transition part between 10 and $30 \mathrm{~nm}$. Studies have shown that for a single nanotube, the thermal conductivity saturates when it reaches $15 \mathrm{~nm}$ long|201]. In this region, we obtained a roughly linear trend 
with the height on the tubes. The derivative of the thermal resistance measured should then compare to $\frac{d R_{\text {bundle }}}{d L}$. Significantly, this derivative does not depend on the tip-sample interface resistance $R_{\text {tip }}+R_{\text {int }}$, contact area and interface contact resistance at the tubes-substrate interface.

An easy and simple model would be to assume thermal isotropy of the layer. However, from the thermal, UFM and SEM images we can see that it is surely not the case. This is also confirmed by the SEM. For our system, we expect a high thermal conductivity along the bundle axis and a low thermal conductivity perpendicularly. This particular form of anisotropy is called orthotropy. In our case, the thermal conductivity has different values in different directions of the material. It is the case for layered material such as graphene but also for the CNTs array as different conductivities parallel to the nanotube axis, $k_{\|}$, and perpendicular to the axis, $k_{\perp}$ are expected. For orthotropic systems, Muzychka et al.105 derived the spreading resistance of a heat source of radius $a$ including the interface resistance $r_{i n t}$ between the material and the substrate. This model is effectively the same as the one for an isotropic layer on substrate presented in chapter 4. However it defines effective quantities $k_{\text {eff }}$ and $t_{\text {eff }}$ replacing the physical ones as follows:

$$
\begin{aligned}
k_{\text {layer }} & \rightarrow k_{e f f}=\sqrt{k_{\|} k_{\perp}} \\
t & \rightarrow t_{e f f}=t \sqrt{k_{\perp} / k_{\|}}+r_{i n t} k_{e f f}
\end{aligned}
$$

From the thermal images, we assume a contact radius $a$ of around $5 \mathrm{~nm}$ (we used the same probe as with the BCP sample of chapter 4). This is also in good agreement with measurements of the silicon tip resistance 89. Then, four fitting parameters can be adjusted to match the experimental data: $R_{t i p}+R_{\text {int }}, r_{i n t}, k_{\|}$ and $k_{\perp}$.

Using both models presented above, we fitted the SThM measurements. The major difference between the two approaches is when considering bundles as cylinders that have only a one dimensional heat transport, no information is obtained on 
the heat dissipation between the bundles. The orthotropic model gives a non-zero thermal conductivity value perpendicular to the bundle axis.

\begin{tabular}{|c|c|}
\hline Model & Bundle thermal conductivity $\left(\mathbf{W m}^{-1} \mathbf{K}^{-1}\right)$ \\
One dimensional & $k_{\text {bundle }}=38 \pm 6$ \\
Orthotropic & $k_{\|}=48 \pm 5, k_{\perp}=8 \pm 1$ \\
\hline
\end{tabular}

Table 6.1: Thermal conductivity obtained with the two models applied.

Applying these two models, we obtained thermal conductivity values, summarised in Table 6.1. Before comparing these results, we note that the interface resistance obtained by the orthotropic model between the nanotubes bundles and the substrate was found relatively low $\left(\sim 1 \times 10^{-10} \mathrm{~m}^{2} \mathrm{KW}^{-1}\right)$. Although, it is important to remember that the orthotropic model considers a uniform layer on a substrate. This does not represent the measured sample, as bundles are not fully covering the surface. As we mentioned above, the estimated coverage is around $27 \%$. Therefore, the interface resistance as well as the thermal conductivity along the bundle direction need to be normalised to this coverage. We thus obtained $r_{\text {int }} \approx 3 \times 10^{-9} \mathrm{~m}^{2} \mathrm{KW}^{-1}$ which is in good agreement with previous studies 201 considering that bundling effects will reduce the interface resistance.

Thermal conductivity values obtained by both models are similar with a slightly higher value along the bundle axis for the orthotropic model. This is coherent as perpendicular transport is also considered. The same reasoning concerning the CNT coverage can be applied here. As thermal conductivity values were obtained through the average thermal resistance measured over bundle height variations, these values need to be scaled by the coverage factor of $27 \%$. However, this only applies to the orthotropic model. Indeed, when considering a one-dimensional heat transport, we consider that the tip contacts a single bundle and measures a resistance variation from the bundle length change. Thus the coverage does not need to be taken into account.

We obtain a thermal conductivity along the bundle axis of $k_{\|}=177 \pm 20$ 
$\mathrm{Wm}^{-1} \mathrm{~K}^{-1}$. Perpendicularly to the bundle axis, no scaling is required as the obtained value of $8 \pm 1 \mathrm{Wm}^{-1} \mathrm{~K}^{-1}$ already reflects the thermal transport occurring between the bundles and as such takes the CNT density into account. If we compute the effective conductivity obtained from the scaled parallel thermal conductivity, $k_{e f f}=\sqrt{k_{\|} k_{\perp}}$, we obtain $k_{e f f}=37 \mathrm{Wm}^{-1} \mathrm{~K}^{-1}$ which agrees very well with $k_{\text {bundle }}=38 \pm 6 \mathrm{Wm}^{-1} \mathrm{~K}^{-1}$ and supports the validity of both models used.

\subsubsection{Low temperature thermal transport}

Using the setup described in chapter 3, we could cool down the cross-sectioned nanotubes forest to a temperature of around $T_{S}=200 \mathrm{~K}$ and perform the same experiment as described already. Low temperature (LT) thermal resistance measured is shown on Figure 6.12 and compared to room temperature (RT) measurements. Interestingly, we observe a giant increase in thermal resistance of almost one order of magnitude. We also note that the silicon substrate appears more resistive as expected and already describe in chapter 3 due to the increase of phonon mean free paths. In this range of temperature, thermal conductivity of single nanotubes or bundled was shown to decrease with decreasing temperature 202 204. Thus, the high resistance measured is expected. We also note that the very small bundles (smaller than $20 \mathrm{~nm}$ ) don't follow the same trend as the longer one. We didn't observe this behaviour at RT and could be linked to the sample BEXP processing which is known to create 1 to $3 \mathrm{~nm}$ of disordered materials at the surface 159.

When turning to modelling, instead of applying the same recipes as before, we assume a one dimensional thermal transport within the bundles but compute the length dependent thermal conductivity from $R_{x}=R_{\text {int }}+L / k A$. For each bundle length $L$, we can obtain its thermal conductivity as

$$
k(L)=\frac{L}{A\left(R_{X}-R_{\text {int }}\right)} .
$$

Again, here we use $A=\pi \times 12^{2} \mathrm{~nm}^{2}$ from the average bundle diameter. To estimate $R_{\text {int }}$ we use data measured at very small height as in this case, $R_{x}$ is dominated 
by $R_{\text {int }}$.

The obtained thermal conductivities are displayed in the inset of Fig. 6.12, Room temperature conductivities appear higher than their LT counterparts. This is due to the high difference in thermal resistance measured and is in line with the thermal conductivity temperature dependence reported in literature 202 204. The absolute values obtained, even if they approach values reported in Kim et al.202 for nanotubes bundles, are nevertheless smaller than generally found in other works. However, these works often report values for single nanotube (single wall or multiwall) and work on single bundles are scarce. A relatively low thermal conductivity for the bundles is expected from as inter-tubes as well as inter-bundles interactions create phonon scattering points and reduce the heat propagation.

Mingo and Broido 205] derived the ballistic thermal conductance of nanotubes. They found that in our temperature range the conductance normalised to the cross-sectional area can be approximated by $\sigma / A \approx 0.6 \times 10^{6} \mathrm{~T}^{3 / 2} \mathrm{Wm}^{2} \mathrm{~K}^{5 / 2}$. Using their model, we plotted the ballistic limit of the length varying thermal conductivity (dashed lines on the inset of Fig.6.12). Higher temperature leads to higher thermal conductivities as we measured. However, the difference between the ballistic limit and our measurement highlights the diffusive resistance of the thermal transport. 


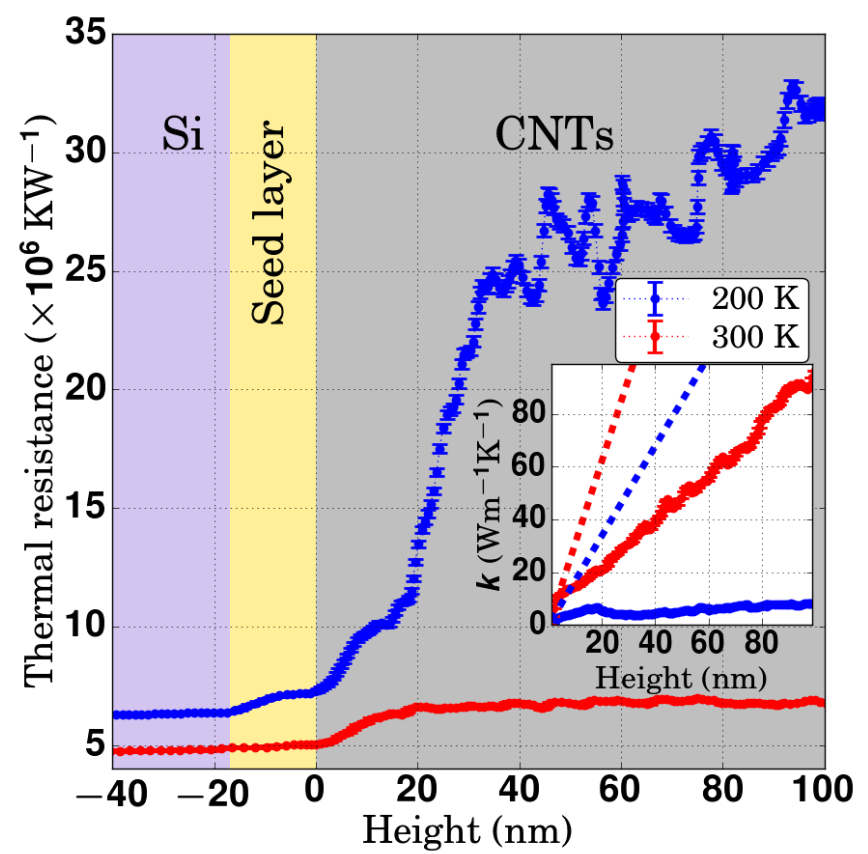

Figure 6.12: Nanotubes bundles thermal resistance as a function of their height for low temperature $(\sim 200 \mathrm{~K})$ and room temperature $(\sim 300 \mathrm{~K})$. Inset: bundle thermal conductivity assuming 1D transport for both temperatures. Dashed lines are the ballistic limits.

A simple way to account for mixed ballistic-diffusive transport is to simply add both components as in $R=R_{\text {ballistic }}+R_{\text {diffusive }}[30]$. This helps to understand the underlying mechanisms behind our results. As temperature reduces, the ballistic resistance and the diffusive resistance increase. Decoupling both effects is difficult as they have the same impact on the measurement. Thus, comparing slopes of the inset of Fig. 6.12 is misleading as it appears that the ballistic contribution to the transport is more important at room temperature. However, this could simply be due to a higher thermal conductivity of the bundles.

In conclusion, we performed measurements on a unique sample composed of length increasing CNT bundles. This sample was prepared by BEXP allowing shallow angle polishing. As the nanotubes kept their structure and alignment after the polishing, this opens new perspectives of materials study. The nanothermal and nanomechanical response was measured using a sharp SThM probe in vacuum. At room temperature, two coherent modelling were applied and obtained an effective thermal conductivity of around $37 \mathrm{Wm}^{-1} \mathrm{~K}^{-1}$. The anisotropy could be estimated 
using an orthotropic model accounting for the thermal conductivity along and perpendicular to the bundle axis taking into account the measure CNT coverage of the sample. Significantly, we found non negligible lateral thermal conductance between the nanotubes bundles. Finally, we compared room temperature to low temperature thermal transport. A drastic increase in the thermal resistance was measured and is related to the reducing CNT thermal conductivity with reducing temperature. Applying one dimensional heat transport simple model, the length varying thermal conductivity could be deduced and compared to the ballistic limit.

This work introduces a new perspective for vertical structures investigations. It also provides information on the temperature dependence of the nanoscale thermal transport of vertically aligned CNT bundles. This has direct implications for thermal interface materials designs and applications. In the next sections, we further study thermal interface materials drawing on similar procedures introduced.

\subsection{Summary}

This chapter reported quantitative SThM measurements of low dimensional systems of $2 \mathrm{D}$ and $1 \mathrm{D}$ materials. For the first time, thermal conductivities and interface thermal resistance of naturally occurring heterostructure franckeite were reported at temperatures between $150 \mathrm{~K}$ and $300 \mathrm{~K} . \mathrm{MoS}_{2}$ on graphene on silicon oxide was also investigated in this temperature range and interface properties were deduced. Then using BEXP, we obtained length varying vertically aligned nanotubes whose thermal conductivities were extracted both along the tubes axis and perpendicularly. With these novel results, we demonstrated SThM advantages for studying nanoscale thermal transport in 1D and 2D materials. 


\section{Thermoelectric phenomena in}

\section{graphene constrictions}

\subsection{Introduction}

This last chapter is dedicated to measurements performed in collaborations with Oxford Materials Department. Results are presented as the collaboration developed: first gold microheaters are measured using SThM for thermometry, then similar devices are tested but with graphene constrictions used as heating sources. This work is thus realised in direct and intense partnership with several persons from Oxford Materials, namely, Achim Harzheim and Pascal Gehring, now at TU Delft.

In the first section, a quantitative estimate is obtained for the temperature distribution created by a gold heater. This estimate is needed to know the temperature of either sides of a graphene constriction as reported in Gehring et al.|21]. Such constrictions are used as a platform to study thermoelectric properties of molecular junctions. We performed measurements at room temperature and at low temperature.

Then, after this preliminary study, another use of these devices was explored. Instead of applying a voltage on the gold microheater, the bias is applied on the graphene constriction itself. That is, current is flowing from one contact to the other through the graphene. With these devices, thermometry SThM is employed to map the temperature distribution. We therefore were able to directly observe with nanoscale resolution the Peltier effect due to the geometry of the graphene 
constriction for the first time. Finally, using the SThM tip as a local heating source, we could map the thermovoltage arising from a local Seebeck effect, establishing a new SPM technique we called Scanning Thermal Gate Microscopy.

These results show the great potential of SThM for thermoelectric studies and thermometry in general. Potential applications are listed in the concluding chapter.

\subsection{Microheater thermometry}

We present results from measurements performed on a graphene junction heated by a $100 \mathrm{~nm}$ thick gold heater. Results are divided in three parts: temperature maps and gradient measurements, null-point determination of the heater temperature and finally, some notes on low temperature thermometry.

\subsubsection{Temperature mapping of the junction heater}

By using the SThM setup in a passive mode, temperature maps could be retrieved from the sample surface. Passive SThM requires the use of a low power bias on the probe sensing element in order to avoid any self-heating. However, as it will be explained later, even with a power of about $0.06 \mathrm{~mW}$, the probe is still slightly heated and this leads to active mode components and, hence, artefacts in the temperature maps. Those artefacts can arise from different topography and materials on the surface. A protruding feature means that the probe is further away from the surface and thus the air gap is bigger which renders a cooler probe even if the actual temperature on the surface is the same. Also, different materials transmit the heat differently to the probe depending on their intrinsic properties. Nevertheless, using a null measurement described below similar to Chung et al. 66], those issues can be fully accounted for during the analysis leading to the absolute temperature measurement results.

We performed 4 scans using 4 different bias voltages on the junction heater: 1.5 $\mathrm{V}, 1.3 \mathrm{~V}, 1.06 \mathrm{~V}$ and $0.75 \mathrm{~V}$. They correspond to 4 power levels: maximum power, 
(x0.75) power, $(\mathrm{x} 0.5)$ power and (x0.25) power. SThM scans are shown in Fig. 7.1 where a calibration is applied to get the weighted temperature of the probe heater. It is to be noted that those values are somewhat approximate as the temperature sensing during the process of scanning is affected by other phenomena such as heat transfer to the probe which are yet to be fully modelled. For example, as the probe scans at a certain speed across the surface, the equilibrium temperature distribution between the sample temperature and the sensor can vary. Similar effects have been observed before, and we believe that the thermal contact is non-trivial during scanning. In order to obtain a quantitative measure of the temperature generated by the heater, we will have to use the force-spectroscopy capability of our AFM machine and to proceed through a null-point method as explained below.
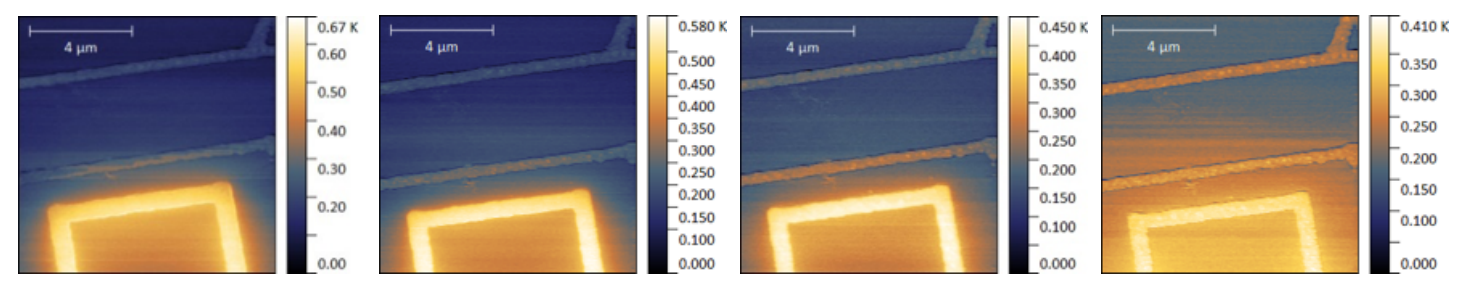

Figure 7.1: SThM temperature maps for 4 different bias voltages on the junction heater. From left to right: $1.5 \mathrm{~V}, 1.3 \mathrm{~V}, 1.06 \mathrm{~V}$ and $0.75 \mathrm{~V}$. The temperature scale on the images is the excess temperature with respect to the surrounding and is a guide value.

The gradient of the temperature distribution can be extracted by fitting the SThM signal along lines on the images. Traces for the four different bias voltages are shown in Fig. 7.2. Performing a linear fit and measuring the slope in the graphene junction region, we find higher slope for the $1.5 \mathrm{~V}$ and $1.3 \mathrm{~V}$ traces and somewhat lower for $1.06 \mathrm{~V}$ and $0.75 \mathrm{~V}$ traces (see Table 7.1). 


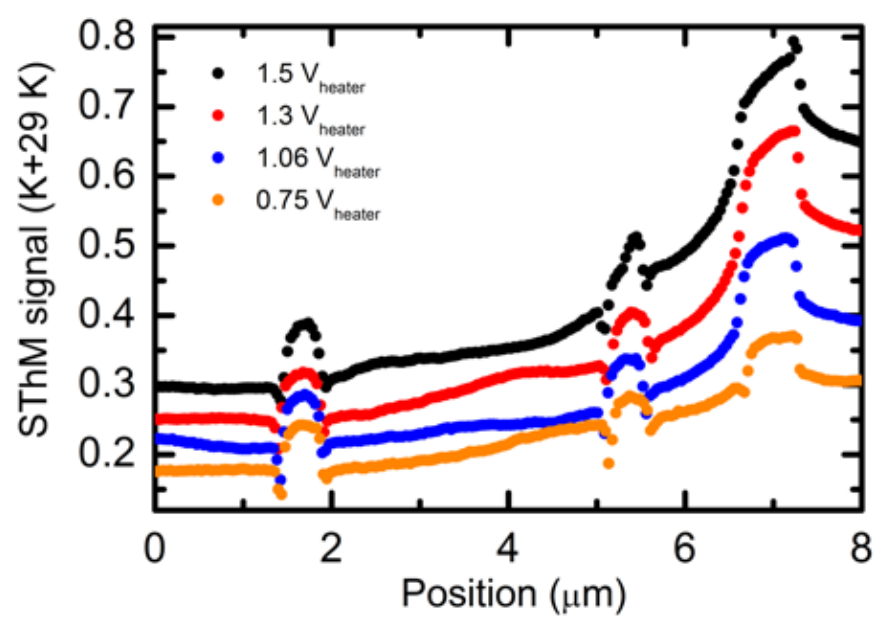

Figure 7.2: SThM traces along the junction direction for various heater voltages. The temperature gradient was estimated in the graphene junction region.

\begin{tabular}{|c|c|c|}
\hline Applied voltage & Linear fit $(m K / \mu m)$ & $\Delta T / \Delta x(m K / \mu m)$ \\
\hline $1.5 \mathrm{~V}$ & $22.7 \pm 0.8$ & $32.6 \pm 0.5$ \\
$1.3 \mathrm{~V}$ & $28.3 \pm 0.6$ & $21.8 \pm 0.3$ \\
$1.06 \mathrm{~V}$ & $13.4 \pm 0.2$ & $20.3 \pm 0.3$ \\
$0.75 \mathrm{~V}$ & $21.1 \pm 0.4$ & $20.4 \pm 0.2$ \\
\hline
\end{tabular}

Table 7.1: Temperature gradient for the 4 bias voltages as obtained by linear fit and slope measurement.

By applying a square waveform to the heater with two different frequencies (14 $\mathrm{Hz}$ and $70 \mathrm{~Hz}$ ), we could test the limits of our detection system and also compare, in the same image, the thermal signal when the heater is modulated. As seen in Fig. 7.3 at $70 \mathrm{~Hz}$ the temperature does not equilibrate as opposed to $14 \mathrm{~Hz}$ modulation. It should be noted that there is evident heating of the wider sample area even where apparently there is no gradient present (strips in the top of the images). The characteristic time corresponds to about $7 \mathrm{~ms}$ (the acquisition and probe time constants were on the order of $1 \mathrm{~ms}$ ). 

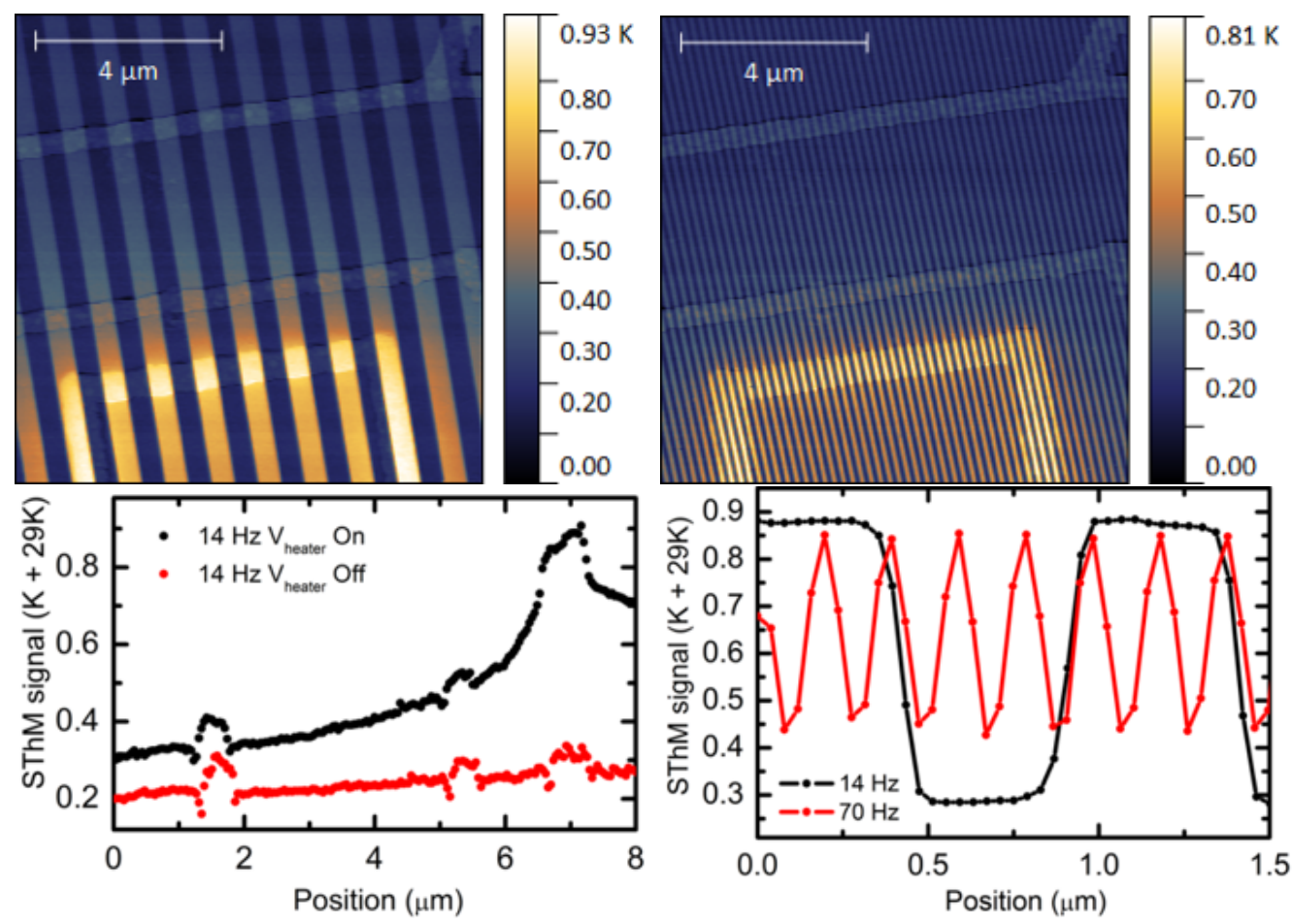

Figure 7.3: SThM images and line traces of $14 \mathrm{~Hz}$ and $70 \mathrm{~Hz}$ square pulses applied to the heater. For $70 \mathrm{~Hz}$, thermal equilibrium is not reached as seen in the SThM signal.

\subsubsection{Null-point measurement of heater excess temperature}

In order to obtain a quantitative estimation of the heater temperature, we used the so-called null-point method. This method consists in measuring the temperature jump between out and in contact for different probe temperatures as well as sample temperatures 66 . For this purpose, spectroscopic SThM curves are recorded. The probe is brought from out to in-contact with the sample and back (Fig. 7.4). As the probe snaps into contacts, a temperature jump occurs due to different probesample temperature. This jump can be processed for different sample and probe temperatures. Results are shown in Fig. 7.4. Temperature jumps measured were always positive $\left(T_{\text {non-contact }}-T_{\text {contact }}\right)$ meaning that the probe was always warmer than the sample even at very low power and with the heater biased. In principle, as we were maintaining the back of the device at $30^{\circ} \mathrm{C}$ and as the back of the probe was around $32^{\circ} \mathrm{C}$, a positive jump is always expected except if the heater temperature is higher than $2^{\circ} \mathrm{C}$. Given the fact that the lowest power we used $(0.06$ 
$\mathrm{mW}$ ) raises the average probe temperature by about $5^{\circ} \mathrm{C}$, in order to get a negative jump, the heater would need to produce around $7^{\circ} \mathrm{C}$ temperature difference. This is in line with the following results.
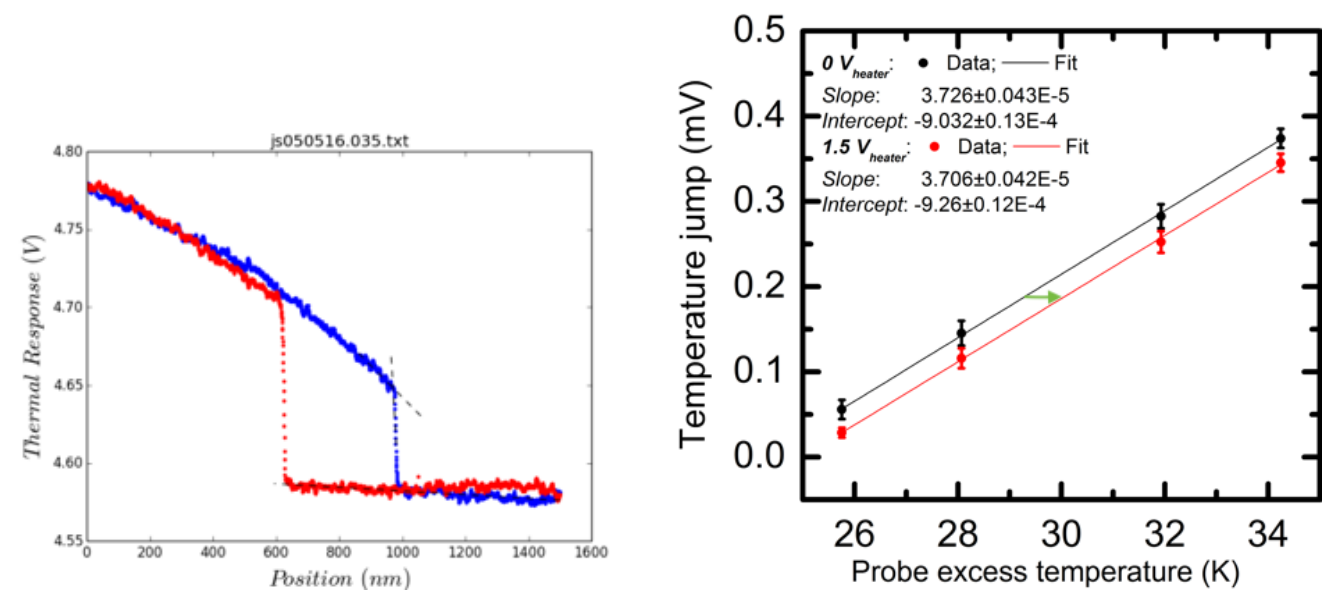

Figure 7.4: Left: example of a typical SThM scan showing approach (blue) and retract (red) SThM signal. Right: temperature jump as a function of probe excess temperature for two heater temperatures. The green arrow highlights the temperature difference sensed by the probe between heated and non-heated sample.

By measuring the probe temperature difference for the same value of temperature jump (shown by the arrow on Fig. 7.4), we obtain a $\Delta T$ of the weighted probe heater temperature that corresponds to a heated and non-heated sample temperature difference. This $\Delta T$ was measured to be $0.8 \pm 15 \% \mathrm{~K}$. The error comes from both the analysis of the temperature jumps (5\%) and the probe excess temperature calibration (10\%). We also need to link the weighted heater temperature difference with the tip temperature. As shown in Fig. 7.5 where a Finite Element simulation of the probe, the temperature distribution in the probe needs to be taken into account. The ratio of the average probe temperature and the tip temperature is found to be about $2.1 \pm 0.1$, giving a tip temperature of $1.68 \pm 0.25$ K. 


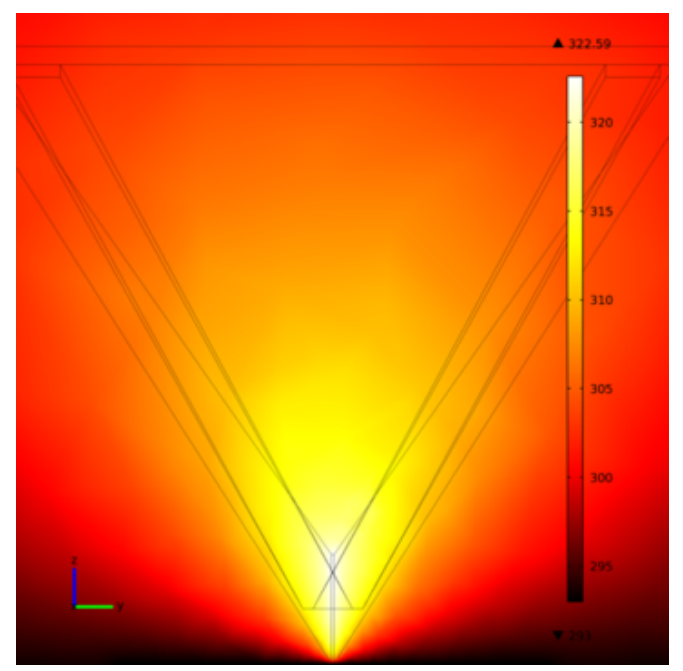

Figure 7.5: Finite element simulation of the SThM probe temperature. Weighted tip heater temperature is around $307 \mathrm{~K}$ that is around $14 \mathrm{~K}$ above the ambient $\mathrm{T}$, whereas the probe tip temperature is around $323 \mathrm{~K}$ that is $30 \mathrm{~K}$ above the ambient T.

\subsubsection{Low temperature thermometry}

Experiments were also performed at low temperature on similar devices. The invacuum SThM measurements were performed in a NT-MDT HV Solver system that operates at pressures $10^{-6}-10^{-7}$ Torr. For precise temperature control the sample was attached to a Peltier heater/cooler stage via a heat conductive varnish; the plate temperature was monitored with a calibrated Pt resistive thermometer, the stage temperature $T_{s t}=T_{0}$ was controlled via PID closed-loop control within $50 \mathrm{mK}$ in the range from $300 \mathrm{~K}$ to $200 \mathrm{~K}$.

We developed here a novel method for quantitative thermometry. The method uses the fact that the total temperature of the sample is the sum of the temperature of sample stage $T_{s t}$ and the heater $T_{s}=T_{s t}+\Delta T_{s}$. Linearity of the SThM response allows then to quantify a temperature increase generated by the micro-heater. Importantly, we implemented the subtraction method in high vacuum conditions, eliminating the through-the-air heat conductance between the sample and the probe, that may provide spurious contrast for topographical variations. The wide temperature range high precision $\mathrm{Pt}$ thermometer for $T_{s t}$ provided results with a total relative error of the temperature increase of $10 \%$. It should be noted that both 
the null and addition methods for determining absolute temperature increase do not depend on the sample-tip thermal resistance due to their differential nature and therefore provide reliable absolute measurements within the error budget specified.
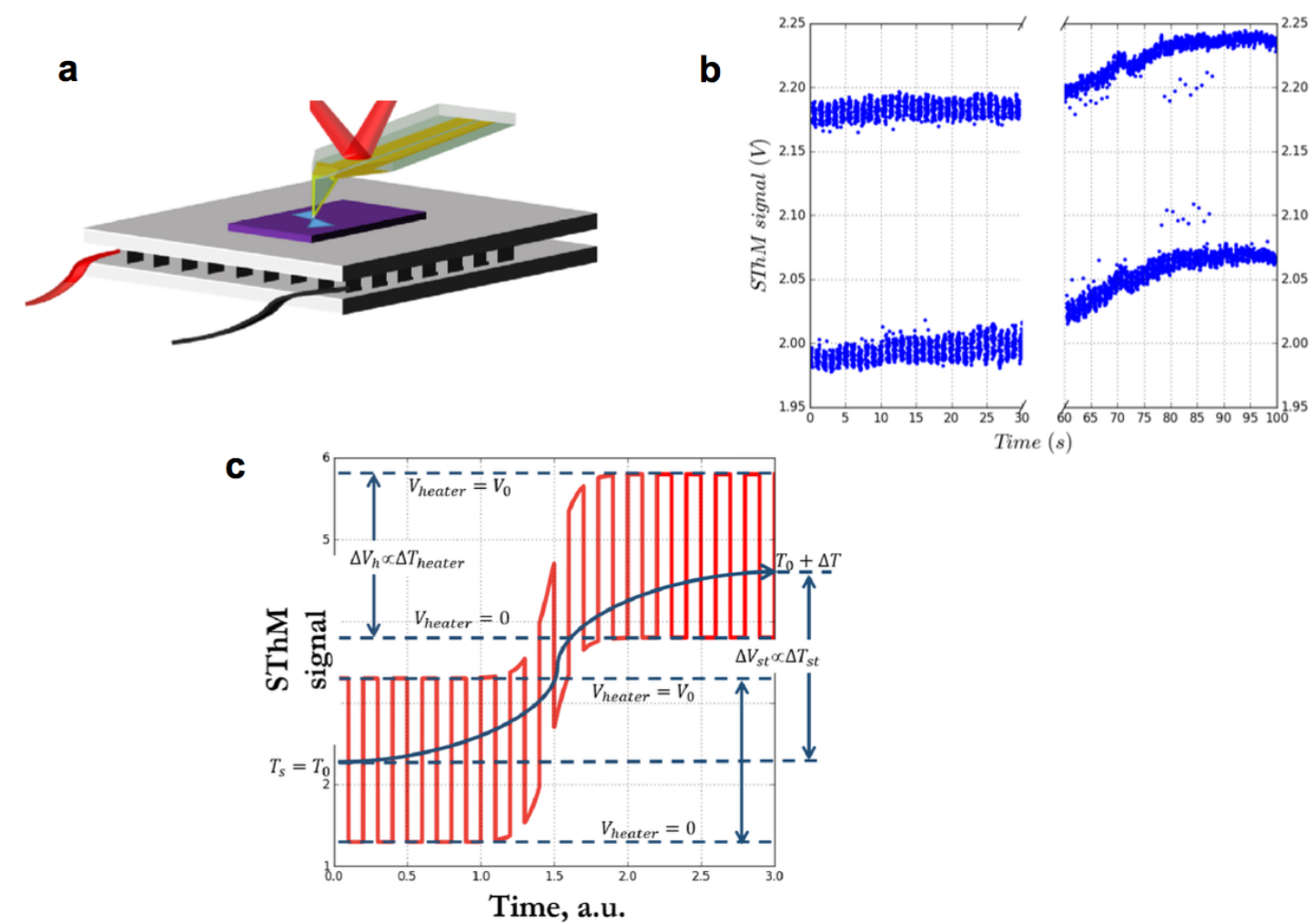

Figure 7.6: (a) Experimental setup with $T$ controlled Peltier stage. (b) Experimental measurement and (c) explanation of the principle of additive approach to the calibration of the absolute rise of the sample temperature due to the heater. A rectangular heating voltage on the heater results in a change of the SThM signal $\Delta V_{h}$, the stage temperature was then increased by $\Delta T_{0}=5 \mathrm{~K}$ leading to a shift of SThM response by $\Delta V_{s t}$.

In this measurement, a rectangular heating voltage on the heater was switched between 0 and $3 \mathrm{~V}$ with a period of $1 \mathrm{~Hz}$ resulting in the change in the SThM signal $\Delta V_{h}$ as shown in the experimental trace in Fig. 7.6 b). The stage temperature was then increased by $\Delta T_{0}=5 \mathrm{~K}$ resulting in a corresponding shift of SThM response by $\Delta V_{s t}$ (see cartoon in Fig. 7.6.). Due to linearity of SThM response, the rise of the chip surface temperature is then found directly as $\Delta T_{h}=\Delta T_{s t}\left(\Delta V_{h} / V_{s t}\right)=$ $5 K\left(\Delta V_{h} / V_{s t}\right)$.

This approach was extended to measurements of the spatial distribution of the heater temperature. For this measurement, the micro-heater was operated at 
about $70 \mathrm{~Hz}$ frequency (that was confirmed to be well below the heater time constant) with a second lock-in amplifier measuring the local heating at this frequency. While this required large time constants of the second lock-in amplifier and long acquisition times of the heating image, the differential approach allowed practically complete elimination of the spurious signals as well as thermal drift. The image in Fig. 7.7 a shows the temperature distribution image of the two heater lines (Fig. 7.7b). We note that, due to its differential nature, this approach allows to map directly the additional surface heating $\Delta T_{h}$. The map shows that the temperature of the silicon oxide area close to the Au heater is about $0.75 \pm 0.05$ of the Au heater temperature which is attributed to the thermal interface resistance between the $\mathrm{Au}$ heater and the substrate. At the same time, for the passive Au line (i.e. the source contact, Fig 7.7 a,b), this thermal resistance does not affect the local temperature of the sample with the passive line showing the same temperature as the surrounding silicon oxide surface. The temperature profiles were compared to finite elements modelling which shows a good agreement between experimentally measured and modelled temperature distributions. The measurements using this method show that, at $300 \mathrm{~K}$, the $3 \mathrm{~V}$ applied to the heater produced $\Delta T_{h}=7.1 \pm 0.4 \mathrm{~K}$ for the $\mathrm{Au}$ heater, while, at $200 \mathrm{~K}$, the temperature was $\Delta T_{h}=10.3 \pm 0.7 \mathrm{~K}$. 
(a)
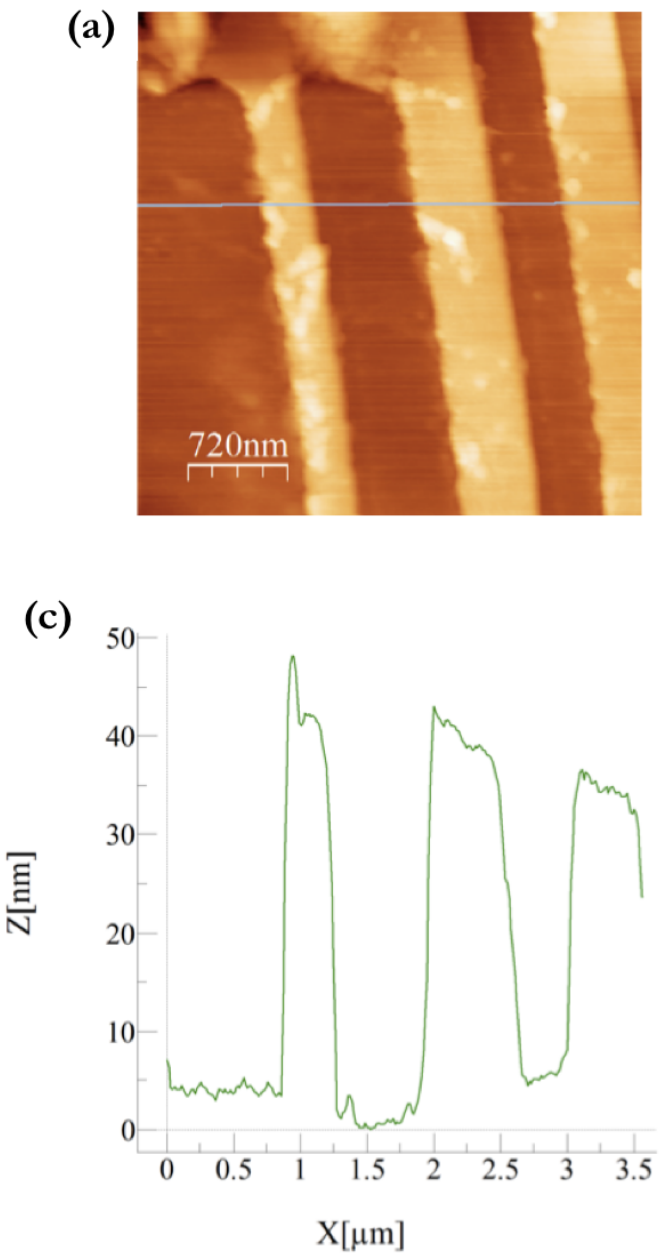

(b)

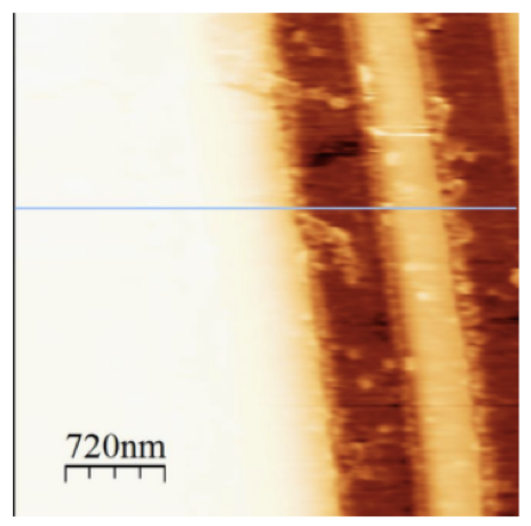

(d)

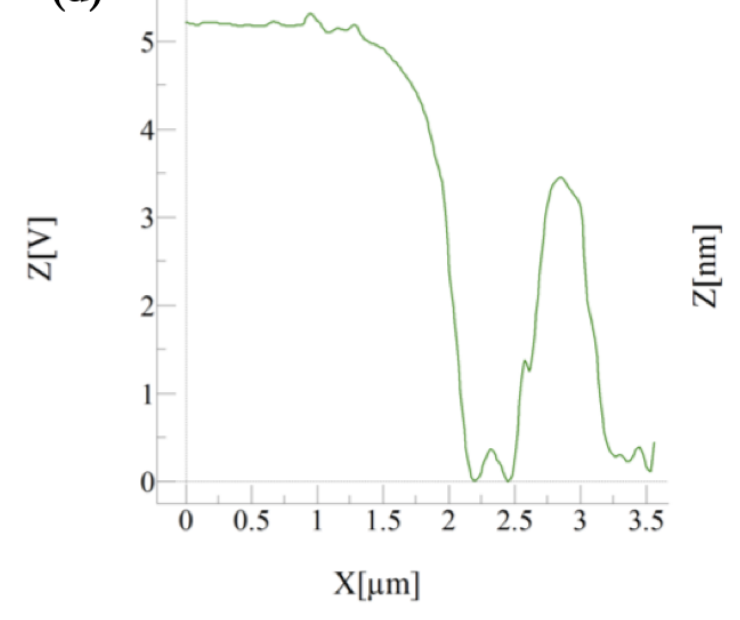

Figure 7.7: Topographical (a) and thermal (b) images of the active heater with the corresponding profiles $(\mathrm{c}, \mathrm{d})$ along the lines shown in the images.

\subsection{Exploring thermoelectric effects in graphene constrictions}

This section is dedicated to measurements performed in continuation of the collaboration established in the previous section. Similar device architectures are investigated but the measurements differ. As previously a temperature map was obtained from the heated gold line, now the temperature field is created by the graphene constriction itself.

If an electrical bias is applied, Joule heating is expected where the current density is the highest, that is, in the constriction itself. Less intuitive is that 
we also obtained thermoelectric related phenomena, e.g. Peltier effect, at the constriction and on its sides.

\subsubsection{Thermometry measurement principles}

To investigate thermoelectric phenomena in electrically biased graphene constrictions, we used measurement principles as proposed by Menges et al. [67, 171]. The idea is to apply an AC bias to the device and measure the first and second harmonic responses of the SThM sensor. The first harmonic corresponds to Peltier effects as $Q=\Pi I$ while the second harmonic corresponds to Joule heating $Q=R I^{2}[67]$. At the same time, the overall SThM tip temperature is elevated and the heat transfer to the sample is recorded as in regular SThM thermal resistance measurements.

The combination of an oscillating sample temperature field and heated probe allows to take into account the variations of the tip-sample conductance which can affect the temperature measured. Menges et al. [67] derived a governing equation for the sample temperature:

$$
\Delta T_{\text {sample }}=\Delta T_{\text {sensor } 0} \frac{V_{A C}}{V_{A C}-V_{D C}}
$$

where $\Delta T_{\text {sensor } 0}$ is the heater temperature out-of-contact, $V_{A C}$ is the $\mathrm{AC}$ variation of the SThM signal at the first or second harmonic and $V_{D C}$ is variation of the regular SThM signal.

The constraint on the measurement is to set an AC bias frequency small enough to always ensure thermal equilibrium in the sample. We estimated that $17 \mathrm{~Hz}$ was satisfying this requirement from inspection of the signal time dependence.

\subsubsection{Scanning Thermal Gate Microscopy (SThGM)}

Another perspective of SThM uses was during these experiments. The entire work presented before in this thesis relied on the ability of SThM to either measure temperature variations or conductance variations. Doing so, we could measure 
local variations of the Seebeck coefficient translating into an intense Peltier effect in the graphene constriction, as shown hereafter. Here, we inverse this picture and obtain the negative of the same image. The SThM probe is used not as a sensor but as a local heater to create a local temperature gradient.

By applying a slow AC bias on the SThM probe, the heater temperature is oscillating at the $\mathrm{AC}$ frequency $\omega$. Then, when contacting a sample, a small portion of the sample volume get locally heated, especially when the sample thermal conductivity is small, as discussed in previous chapters. If, then, contacts are present, as in the graphene constriction devices, the voltage at the contacts can be measured precisely. This voltage arises from similar thermoelectric effects (Seebeck effect) that we observed already in the Peltier effect measurements.

Using a high-impedance amplifier and a lock-in, we measure the voltage at frequency $2 \omega$ which directly links to the thermovoltage from

$$
S=-\frac{\Delta V}{\Delta T}
$$

where $\Delta T$ is the local temperature gradient created by the probe, $S$ the Seebeck coefficient of the material and $\Delta V$ the thermovoltage created by the temperature gradient.

\subsubsection{Main results}

The first investigated geometry was the same as the one presented above, a bowtie shaped graphene constriction. The space between the two contacts is about $4 \mu \mathrm{m}$ and the narrowest width in the middle of the bowtie is around $100 \mathrm{~nm}$. A typical topography image is shown on Fig. 7.8. We note that resist residues were often observed on the samples even after a prolonged annealing of the chip. However, these residues can usually be removed by scanning the device several times with the SThM tip. The sample of Fig. 7.8 is a few layer graphene sample. Monolayer sample were also investigated and presented similar features. 


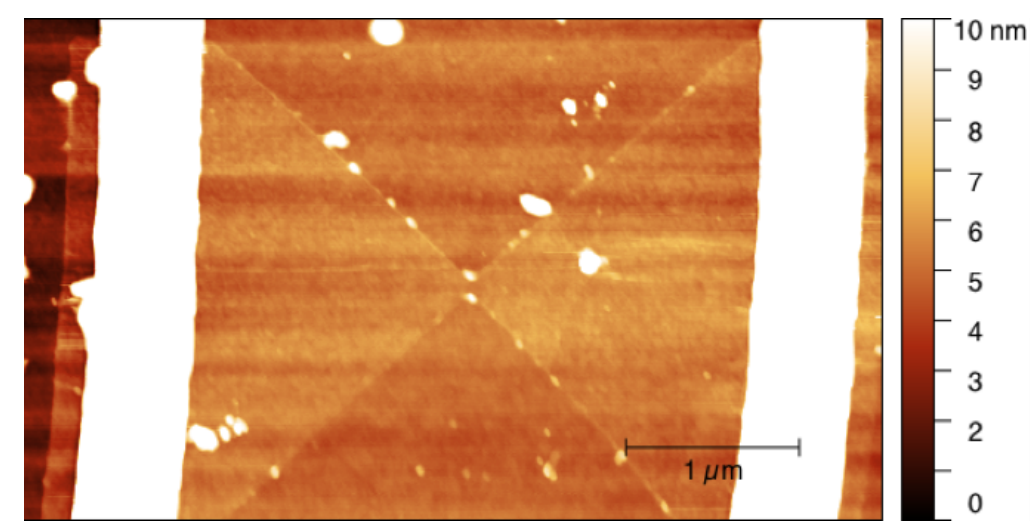

Figure 7.8: Topography of a few layers graphene bowtie constriction.

We then measured the Peltier and Joule signals when applying an AC bias on the graphene device. Due to the low frequency ensuring thermal equilibrium, the SThM rate was set low enough so that several periods are measured at each point and fed into the lock-in amplifier. Therefore, each image presented below represent between 2 and 4 hours scanning. Over such a time scale, temperature variations of the system can greatly affect the measurements, especially the thermal drift of the piezo positioning. However, the room was kept at a constant temperature minimising this drift.

Figs. 7.9 show the Peltier and Joule images received from the bowtie constriction. The striking observation is the butterfly-like shape of the Peltier response with positive and negative signal. This change of sign corresponds to a $180^{\circ}$ phase change of the first harmonic signal. The Joule images can be easily interpreted as the current density, creating proportionally a Joule heating which is expected to be higher in the center of the constriction. 

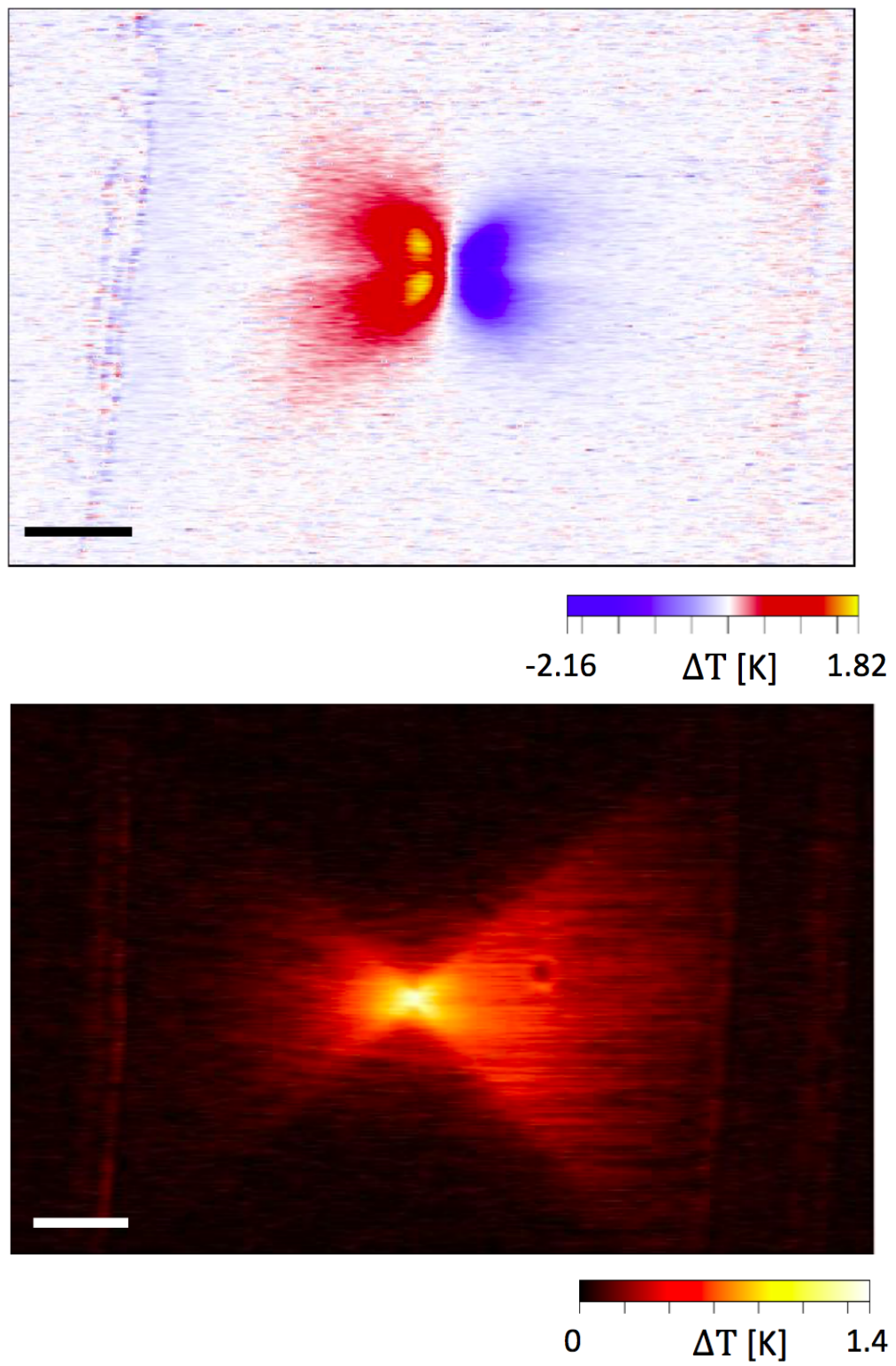

Figure 7.9: Peltier and Joule heating images of a few layers graphene bowtie constriction. Scale bar is $500 \mathrm{~nm}$.

Before discussing the origin of this Peltier signal, we show the corresponding picture obtained from Scanning Thermal Gate microscopy. Fig. 7.10 shows the thermovoltage arising from the hot tip scanning on the sample. The same butterflylike shape response is obtained. We also note another sign change in the left side of the constriction. The biggest signal change occurs near the constriction and not close the electrodes. Electrodes doping has been reported and Peltier effect at the electrodes already observed [57, 206]. 


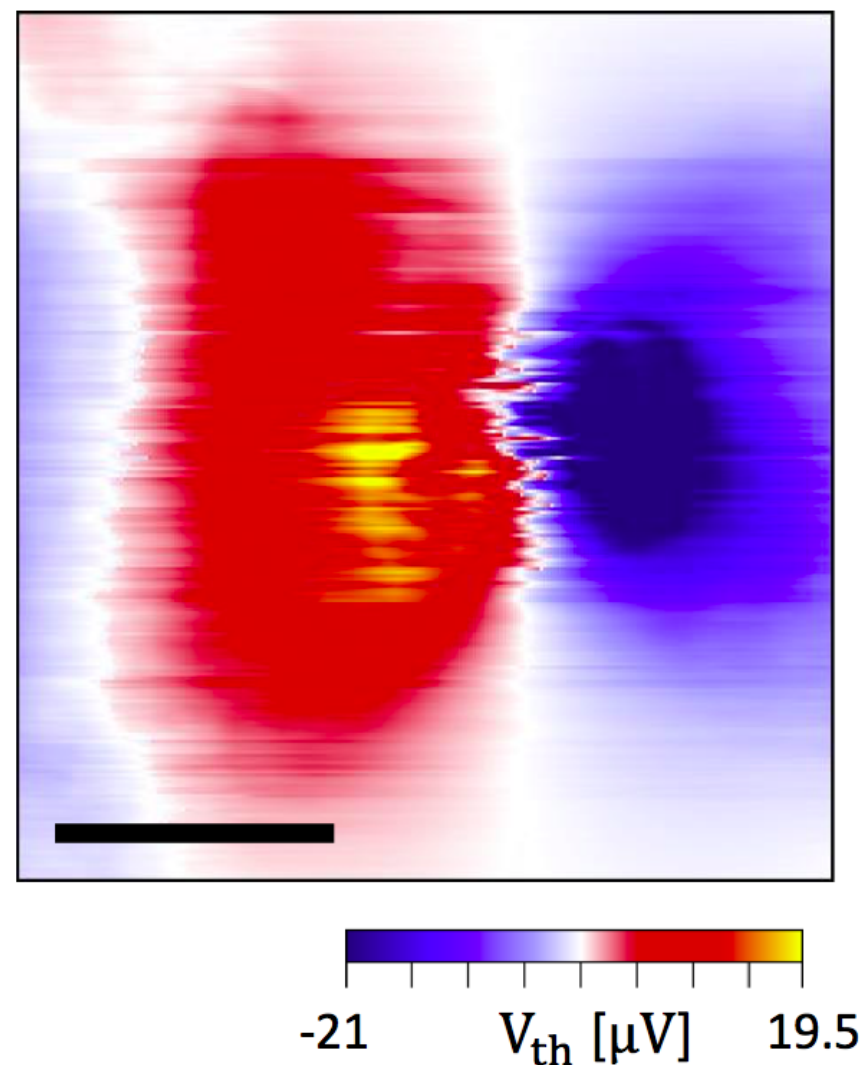

Figure 7.10: Thermovoltage map of a few layers graphene bowtie constriction. Scale bar is $500 \mathrm{~nm}$.

The origin of such behaviour is still under discussion. We suspect a geometry effect on the Seebeck coefficient of the graphene. For example, if the electron mean free path is affected by the geometry, the Seebeck coefficient is likely to change. As such, an effective thermocouple junction would be formed on either side of the constriction. Another possible explanation could be the Thomson effect, that is temperature dependence of the Seebeck coefficient. However, the range of temperature change is small and, thus, the Seebeck coefficient change on temperature is most likely negligible. Also, as the thermovoltage map shows similar effects without the presence of any electrical current, we can rule out this explanation.

In order to investigate further this geometric effect, we then measured two different geometries as summarised in Fig. 7.11. In the first one, a long nanoribbon was patterned between two large graphene pads. The second one connects the large graphene pads via three nanoribbons through two islands. This second geometry 
will rule out the possibility of contact doping and enhance the fact that the Seebeck coefficient change comes from the material geometry itself.

\section{Long ribbon}

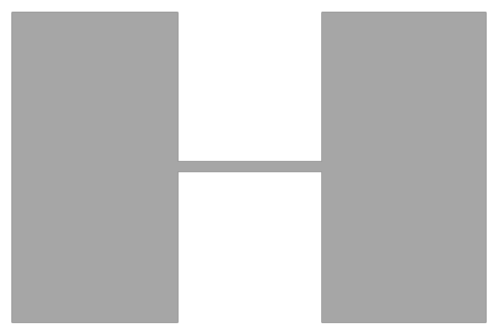

Multiple islands

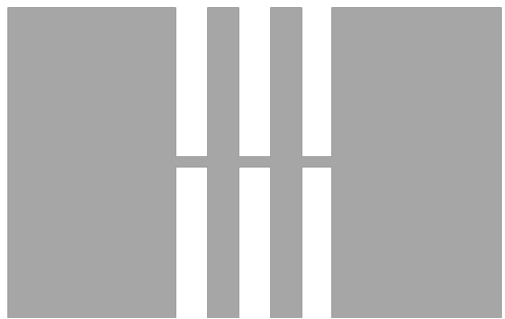

Figure 7.11: Topography and Joule heating images of a few layers graphene bowtie constriction.

We investigated first the long ribbon using the same setup as for the bow tie. Figs. 7.12 show Peltier, Joule and thermovoltage images. As expected, Joule heating occurs over the whole ribbon. The Peltier image displays several sign changes along the ribbon. The same is observed on the thermovoltage map. While in the bow tie geometry, we could postulate a geometric effect on the Seebeck coefficient, in the nanoribbon, as the geometry is fairly constant along the ribbon, such hypothesis does not hold. However, edge doping and charge puddles [57] are likely to be responsible for the response obtained.

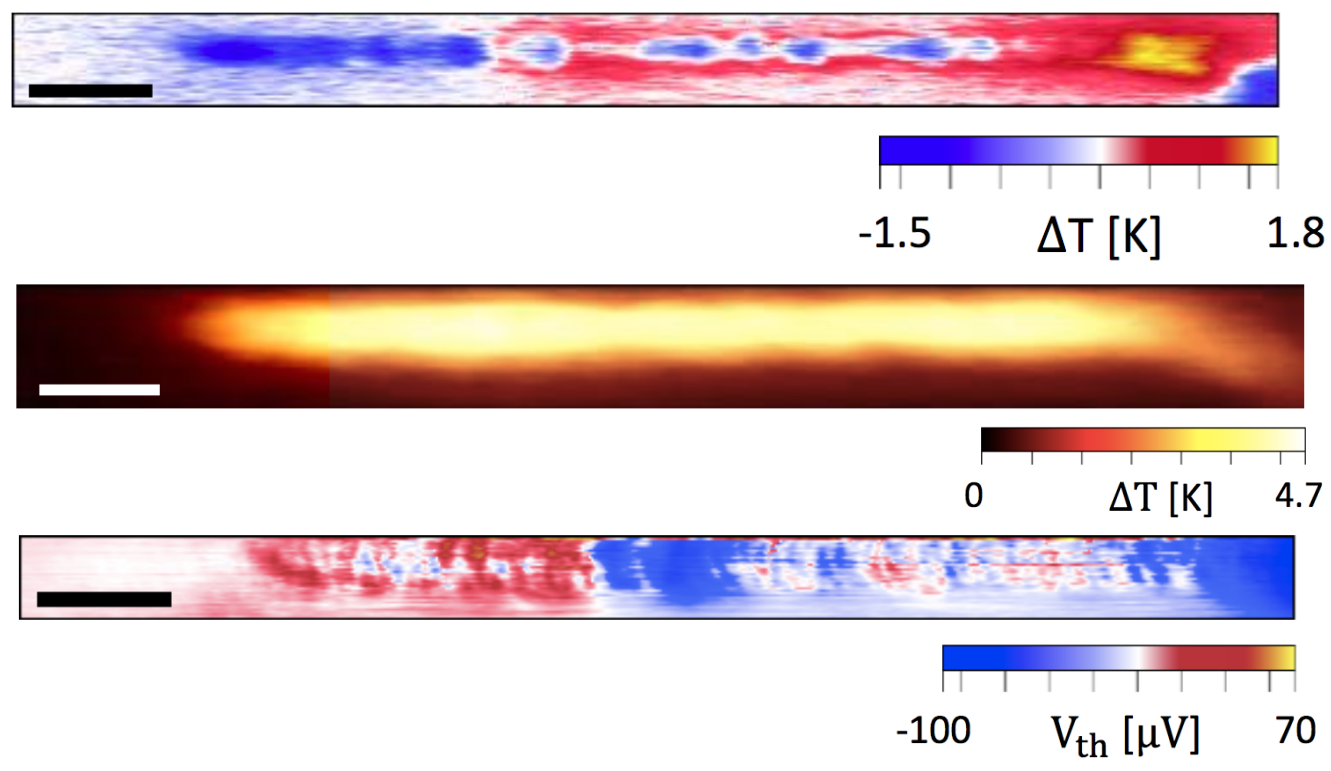

Figure 7.12: Peltier, Joule heating and thermovoltage images of the long ribbon geometry. Scale bar is $200 \mathrm{~nm}$. 
The last geometry investigated consists of three nanoribbons connecting large islands. Figs. 7.13 show the Peltier, Joule and thermovoltage maps obtained from this device. As we observed, each constriction between an island and a nanoribbon displays a sign change in the signal. Furthermore, the amplitude of this sign change is similar for each constriction. With this observation, doping from the contact is ruled out as we would otherwise obtain different amplitudes at each constrictions. Similar features are observed on the thermovoltage map. Thus, the effect is arising from the graphene geometry itself as we hypothesised above.
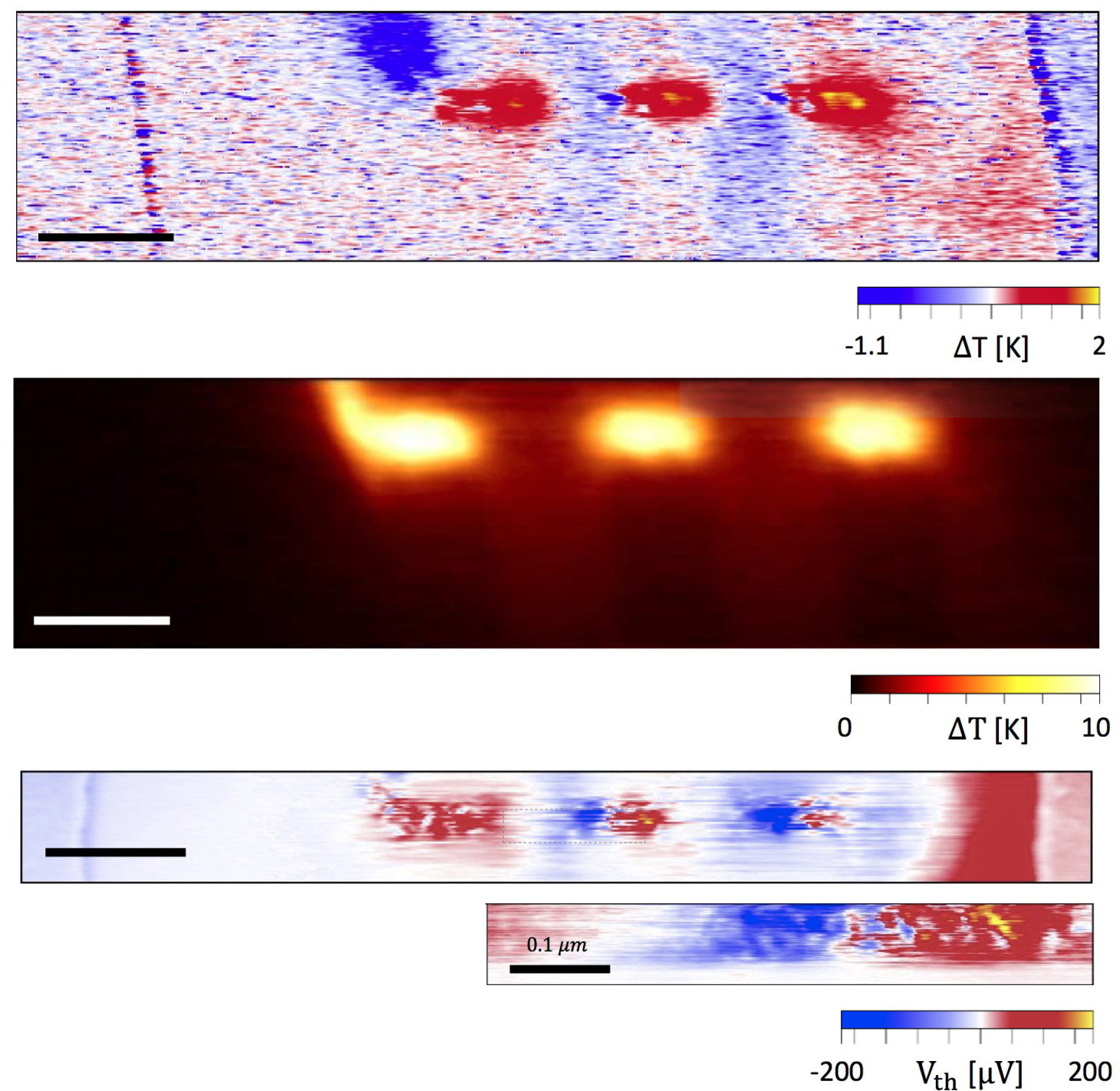

Figure 7.13: Peltier, Joule heating and thermovoltage images of the mutliple islands geometry. Scale bar is $500 \mathrm{~nm}$. 


\subsection{Summary}

With the SThM thermometry capabilities, we could map the temperature distribution arising from a gold line heater. This was realised both at room temperature and at low temperature and allowed the knowledge of the temperature gradient across molecular junctions investigated for thermoelectric applications.

Then, based on the experience acquired in these first experiments, we develop a three-terminal approach to study thermoelectric phenomena in nanoscale devices. This three-terminal setup consists of the SThM tip acting either as a temperature sensor or as a temperature gradient supply and two electrical contacts for either current supply or thermovoltage sensing.

With this three-terminal approach, we could unveil new thermoelectric phenomena in graphene devices. Geometrically shaping the graphene creates Peltier and Seebeck effects. Theories and models are needed to fully understand such results which open new perspectives for 2D materials uses as thermoelectric energy converters. 


\section{Conclusion and perspectives}

SThM has been proven to be a successful tool to assess nanothermal phenomena. However, performing meaningful comparison and understanding the output of an experiment remained a challenge due to the versatility and diversity of SThM practices. Therefore clear definitions and measurements models are needed to better analyse results, enhance reproducibility and create a framework of understanding.

The first focus of this thesis was in line with this goal as we developed experimental and analytical tools achieving stable and comparable results. Then, applying this framework to various materials, we extracted thermal properties directly from the SThM measurements. We summarised here the main outcomes of our work.

\subsection{Major achievements of this thesis}

- Comprehensive model and experimental setup for high precision SThM

Understanding the heat transfer in a distributed heater was essential to extract thermal resistance arising from materials probed. We achieved better understanding of measurements and avoided numerous systematic errors usually ensuing from the lack of such a model. In parallel with analytical developments, experimental errors from the unstable environment were unveiled. Drifting surrounding temperatures and laser related uncertainties were the major sources of errors. The measurement cell temperature is controlled via feedback loop using Peltier devices, thus eliminating thermal drift 
and improving the precision up to 10 fold. We note that this setup could also be used for many other studies such as thermoelectrics or any temperature related phenomena. Experiments without laser were performed and showed to greatly increase stability and reproducibility.

\section{- cryoSThM}

SThM measurements could be performed for the first time at low temperatures by thermally connecting the sample holder in vacuum to an external liquid nitrogen dewar. We achieved temperatures below $150 \mathrm{~K}$. We developed a simple model to account for the low sample temperature and extract sample related properties.

\section{- Nanothermal probing of materials in three dimensions}

We showed the possibility of performing 3D investigations of nanothermal properties through the combination of BEXP and SThM. This combination also allows to extract thermal properties such as thermal conductivity. This contrasts with standard techniques facing challenges with nanoscale systems. Adaptable to a wide range of materials, BEXP suits particularly well the SThM needs of well defined surfaces. By 3D probing SiGe and GeSn alloys, we could assess the anisotropy of such materials. Moreover, sample processing effects were proposed as responsible for a decrease in nanoscale heat conductance. This is of major interest in devices fabrication and testing as failure often arise from heat management aspects.

\section{- Quantitative thermal transport in low dimensional materials}

We measured for the first time temperature-dependent thermal conductivity of naturally occurring heterostructure franckeite and its interface resistance with silicon oxide using our cryoSThM setup. We also studied the $\mathrm{MoS}_{2^{-}}$ graphene interface. Our results provide crucial insights for the realisation of devices using 2D materials. Furthermore, we created an array of CNTs bundles with increasing length and extracted their anisotropic thermal con- 
ductivity at low and room temperature. These arrays are currently developed to act as heat spreading materials and their utility is assessed by our results.

\section{- Dynamic SThM quasi non-contact method}

A non negligible realisation is the combination of the UFM quasi non-contact principles with SThM measurements to create a dynamic SThM method. Non-contact SThM methods are needed for soft matter probing and our method provide a simple approach allowing simultaneous nanothermal and nanomechanical experiments both in air and in liquid.

\section{- New thermoelectric phenomena in nanostructured graphene}

Finally, we investigated new thermoelectric systems by adopting a threeterminal approach. This method allows a double-sided understanding of the same system. The thermal and electrical sides are both unveiled using the same setup. We obtained thermoelectric junctions formed by graphene nanoconstrictions thus implying that the Seebeck coefficient varies with geometry. These findings open new possibilities for thermoelectric energy conversion.

\subsection{Perspectives and challenges for nanoscale ther- mal measurements}

While working on this thesis, many SThM advances were realised in various groups creating a dynamic nanothermal community. However, SThM hurdles will always remain present as in any scanning probe microscopy technique. As we believe is clear from our work, when one performs SThM experiments, one actually studies three systems: a given sample, measurement instrument and the combination. We think that seeking an SThM black box, similar to standard techniques providing directly material properties, is like entering a maze. The numerous factors and parameters participating to an SThM experiment, even without considering the various SThM techniques, create as many difficulties to create SThM standards. 
Nevertheless, non-standard techniques have great potentials as they have the possibility to discover non-standard properties. Many materials thermal properties are to be explored at the nanoscale both experimentally and through modelling. SThM is the ultimate tool to study nanothermal phenomena. When used for the right purpose, quantitative outcome is possible. The question of the adequacy between the object and the tool is fundamental and requires a very deep understanding of both the tool and the object. A screw driver does not brew tea, but it balances a Wheatstone bridge. 


\section{A. Appendices}

\section{A.1 Estimation of the contact radius}

Two typical methods are used to estimate the contact radius, crucial parameter to obtain quantitative values from SThM measurements. The first method uses SEM imaging of the tip apex as on Fig. A.1 that we used for the 2D materials heterostructure measurements and bulk samples at cryogenic temperatures.

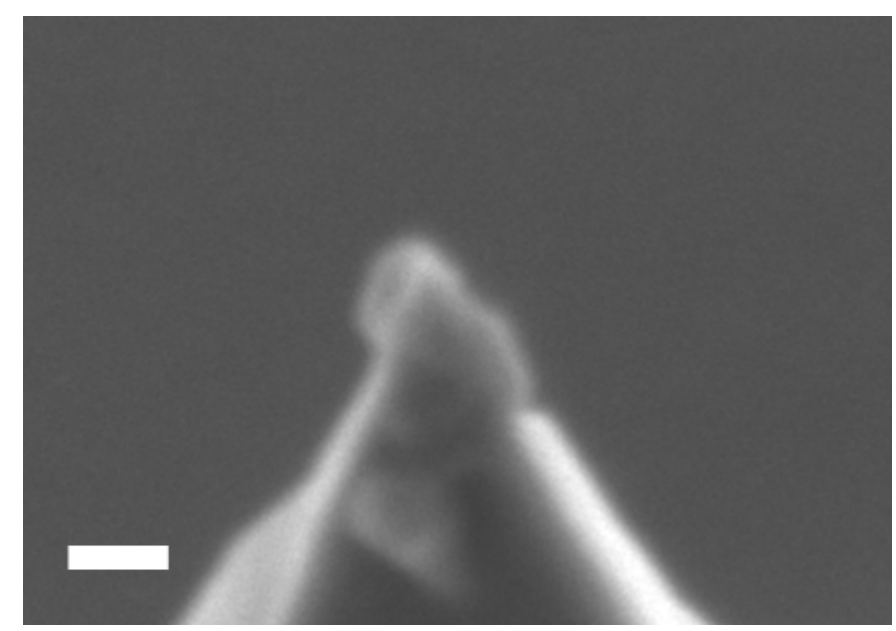

Figure A.1: Doped silicon tip SEM image used for estimation of the contact area. Scale bar is $200 \mathrm{~nm}$

The other method is based on inspection of the thermal image as explained by Menges et al.[113]. If the topography change between two regions is minimal (ideally no topography variation), the change of the thermal signal can be used to estimate the contact radius. We used this method with the BCP samples and hence with the vertically aligned nanotubes. 


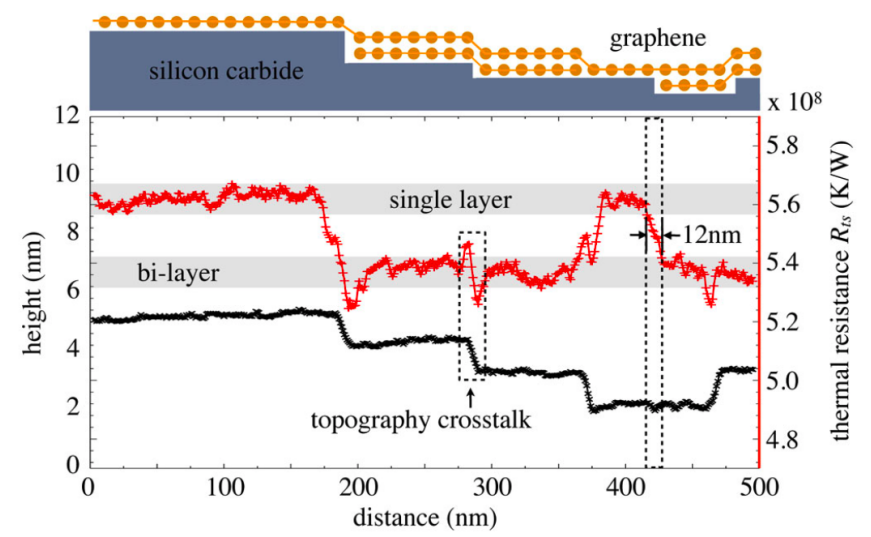

Figure A.2: Comparison of topography and thermal signal to estimate the tip contact radius from $[113$.

We note also that samples like the silicon oxide steps or the BEXP crosssectioned silicon oxide on silicon can also provide estimation of the contact radius as we showed in chapter 3 and 5 .

\section{A.2 Conical tip temperature distribution model}

In order to model the temperature distribution in the doped silicon conical tip, we assume the following:

- a truncated conical tip of known apex radius, $r_{0}$, half-angle $\alpha$ and length $l_{t}$;

- diffusive transport in the whole tip;

- power $Q$ flowing uniformly through the tip.

The tip model is represented on Fig. A.3. 


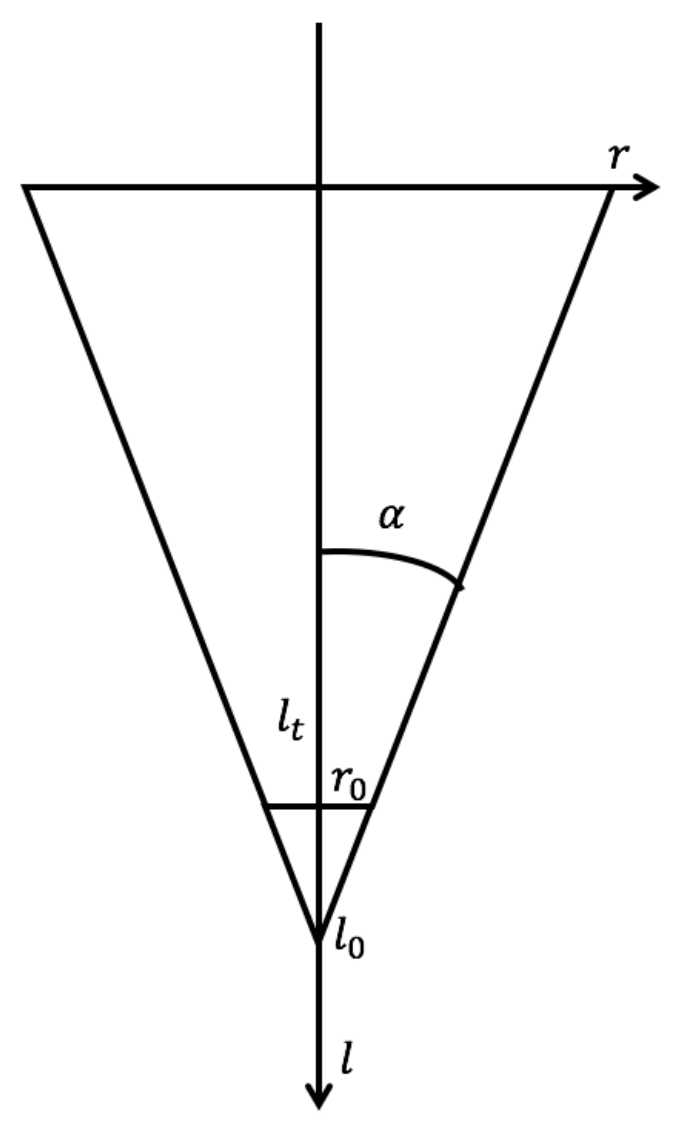

Figure A.3: Schematic of the conical tip.

The heat equation can be written in the case of $1 \mathrm{D}$ heat conduction as

$$
Q=-k A(l) \frac{d T}{d l}
$$

where $Q$ is the rate of heat conduction, $k$ is the thermal conductivity of the material and $A(l)$ is the section of cone at the position $l$.

From trigonometry, we have

$$
\tan \alpha=\frac{r_{0}}{l_{0}}=\frac{r}{l_{t}-l+l_{0}}
$$

Thus, $A(l)$ is given by

$$
A(l)=\pi r^{2}
$$

In order to get the temperature distribution in the tip, we need to integrate 
the heat equation

$$
\int_{T=T_{0}}^{T(l)}=\int_{l=0}^{l}-\frac{Q}{\pi k\left(\left(l_{t}-l\right) \tan \alpha+r_{0}\right)^{2}} d l
$$

This gives

$$
T(l)=T_{0}-\frac{Q l}{\left(\left(l_{t}-l\right) \tan \alpha+r_{0}\right) \pi k\left(l_{t} \tan \alpha+r_{0}\right)}
$$

with $T_{0}$, the temperature at the heater side.

By defining $Q$ from the heat flowing from the heater to the sample through the whole tip:

$$
Q=\frac{\left(T_{\text {heater }}-T_{\text {sample }}\right) r_{0} \pi k\left(l_{t} \tan \alpha+r_{0}\right)}{l_{t}}
$$

we can plot the temperature distribution within the conical silicon tip.

\section{A.3 Description of experimental setups}

In this section, we describe briefly the SThM experimental setups used in this thesis. First, general SThM components and uses are described. Then SThM box comprising the modified Wheatstone bridge is introduced and finally we explain laser management in our different systems.

\section{A.3.1 General SThM setup components and uses}

Fig. A.4 shows the various components of the SThM. 


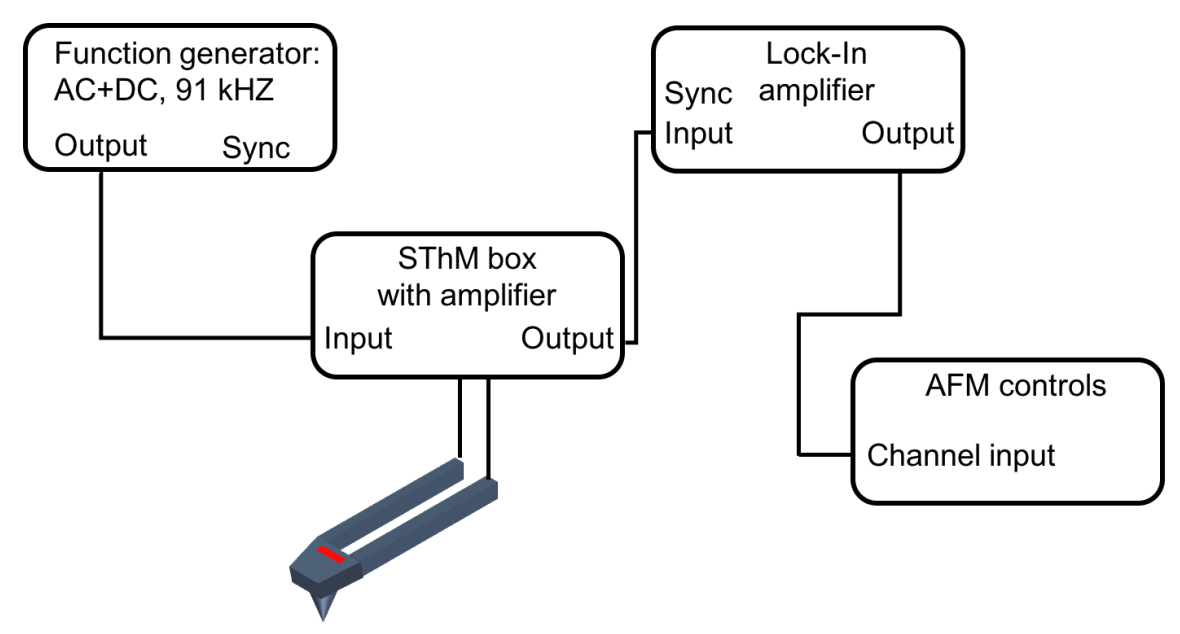

Figure A.4: Principals SThM components.

Many SThM setups are possible. Some group work with constant probe temperature, while others with constant power applied, with DC load or AC load. In Lancaster, we work with constant applied power and a combination of $\mathrm{AC}+\mathrm{DC}$ bias.

Constant power means that the bias on the probe is fixed. As power is defined by $P=V^{2} / R$, and changes in the resistance are small, then the power is constant. The assumption on the resistance is satisfied because resistance variations experienced by the probe during experiments are tiny.

The voltage bias on the probe is composed of an AC part and a DC part. The $\mathrm{AC}$ component, at $91 \mathrm{kHz}$ provides a high signal-to-noise ratio and is detected by a lock-in (Stanford research, SR-830). This alternating bias also creates some selfheating of the probe as we explain hereafter. The DC part provides self-heating. Care must be taken in the applied load as the current should not be higher than $1.5 \mathrm{~mA}$ for the KNT probe.

When balancing the bridge, a small power is applied on the probe in order to minimise the self-heating. Typically, we use $0.1 \mathrm{~V}_{p p}$. In regular operations, $4 \mathrm{~V}_{p p}$ provide a nice signal combined with 2 or $3 \mathrm{~V}_{d c}$ for the KNT probe. Same AC signal can be used with the doped silicon probe but the DC offset depends on the desired probe temperature. 


\section{A.3.2 SThM box}

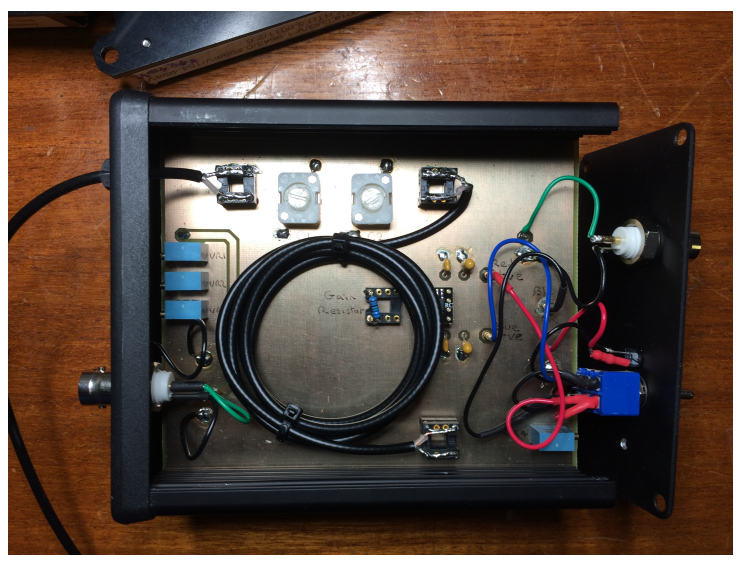

Figure A.5: SThM box with precise amplification.

The circuit inside the SThM box is presented in Fig. A.6. To resistors (R2 and R3) and two capacitors (C1 and C2) are used to balanced the bridge in standard operations. Usually R4 is set at the same resistance as the probe when the probe is changed. In the best scenario, R4 is checked from time to time while using the same probe. One of the major design tricks to balance the balance the capacitance of the bridge is to have use two cables of the same length $(75 \mathrm{~cm})$ to connect the probe and R4. Note that in the vacuum system, as extra connections and cables are needed between the SThM box and the probe, we soldered an extra capacitor in parallel with $\mathrm{C} 2$.

The main advantage of this SThM box is that it includes an ultra-low noise amplifier. No precise assessment of the noise reduction was performed but from experience, we could clearly observe an improvement in the signal-to-noise ratio between the new version of this SThM and the previous one. Even with very low amplitude signal, variations of $d V / V$ and on images are observed.

Some practical tips for the use of this SThM box:

- Batteries: if using rechargeable batteries, with a heavy use, they last around one to two months. Strange signals might arise from low batteries, even if the LED is still bright on.

- R1 is used to limit to current applied on the probe and therefore its value 
depends on the the probe used. It is typically adjusted by measure the "box ratio": apply $1 \mathrm{~V}_{d c}$ on the box input and measuring the voltage across the probe (or a similar resistance fixed resistor). This ratio should be around 0.1: if applying $1 \mathrm{~V}_{d c}$, the multimeter should read $0.1 \mathrm{~V}_{d c}$. Once $\mathrm{R} 1$ is fixed, it shouldn't be changed.

- When balancing the bridge, for a new probe, first R4 should be adjusted at the probe resistance value. This requires to open the SThM box and is not very user-friendly. Then, R2 and R3 can be adjusted. R2 has a greater effect on the balance while R3 is for fine tuning.

- The capacitors are crucial to balance the bridge. To achieve a nice bridge balance, a good iterative process is to adjust the resistors to minimise the signal then to adjust the capacitors and to repeat this sequence.

- The SThM box components are soldered on a PCB board. They need to be manipulated with care. Especially the resistors R2 and R3 which can detach if strong forces are applied with screw drivers. 


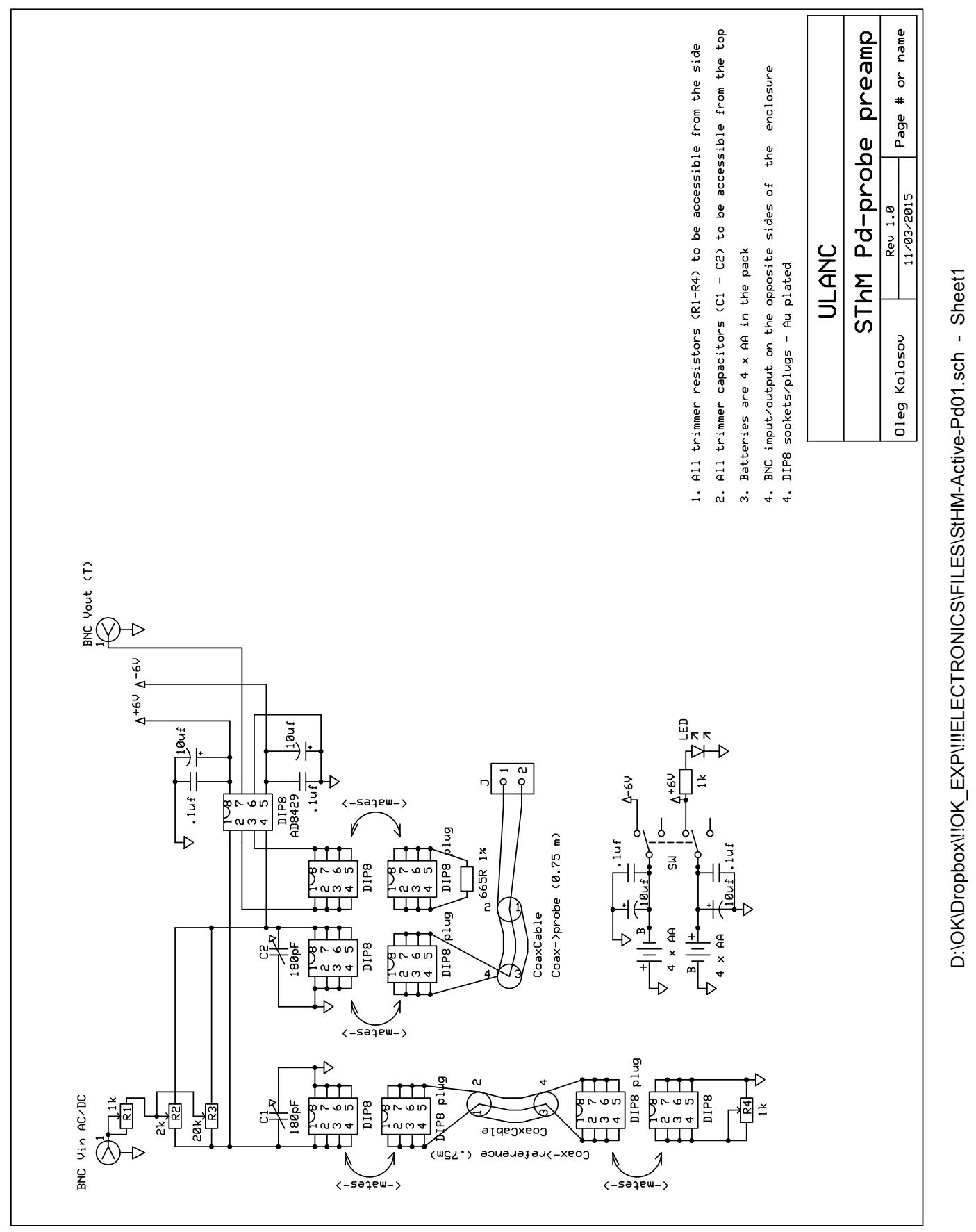

Figure A.6: SThM box circuit with preamplifier. 


\section{A.3.3 Laser management}

As explained in chapter 3 , the laser illumination on the back of the probe causes reproducibility and stability issues on SThM measurements. When taking point measurements, we then decided to avoid this illumination.

In air, the Bruker system does not allow to switch of the laser while keeping the piezovoltages active. Therefore, we used a blocking paddle (see Fig. A.7) to stop the laser illuminating the probe when necessary. Note that, when using the KNT probe, the deflection of the probe is affected by the laser heating.

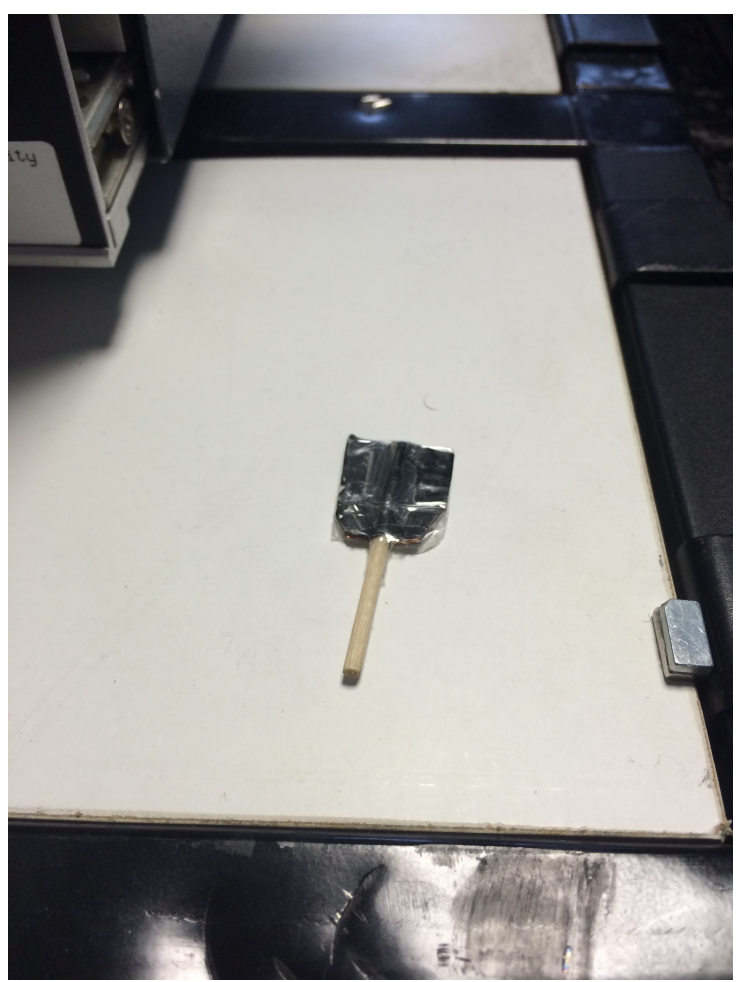

Figure A.7: Laser blocking nanopaddle

In the vacuum system, the NT-MDT software allows to switch the laser. However, this has the effect to turn the feedback on the z-position off. Then, the approach the probe from the surface, the motor must be used carefully, step by step. 


\section{A.4 Python code for approach-retract curve anal- ysis}

We developed a Python code to analyse approach-retract SThM measurements. This code, while fully functional, requires some practice in order to understand the outputs and important parameters to achieve the best analysis.

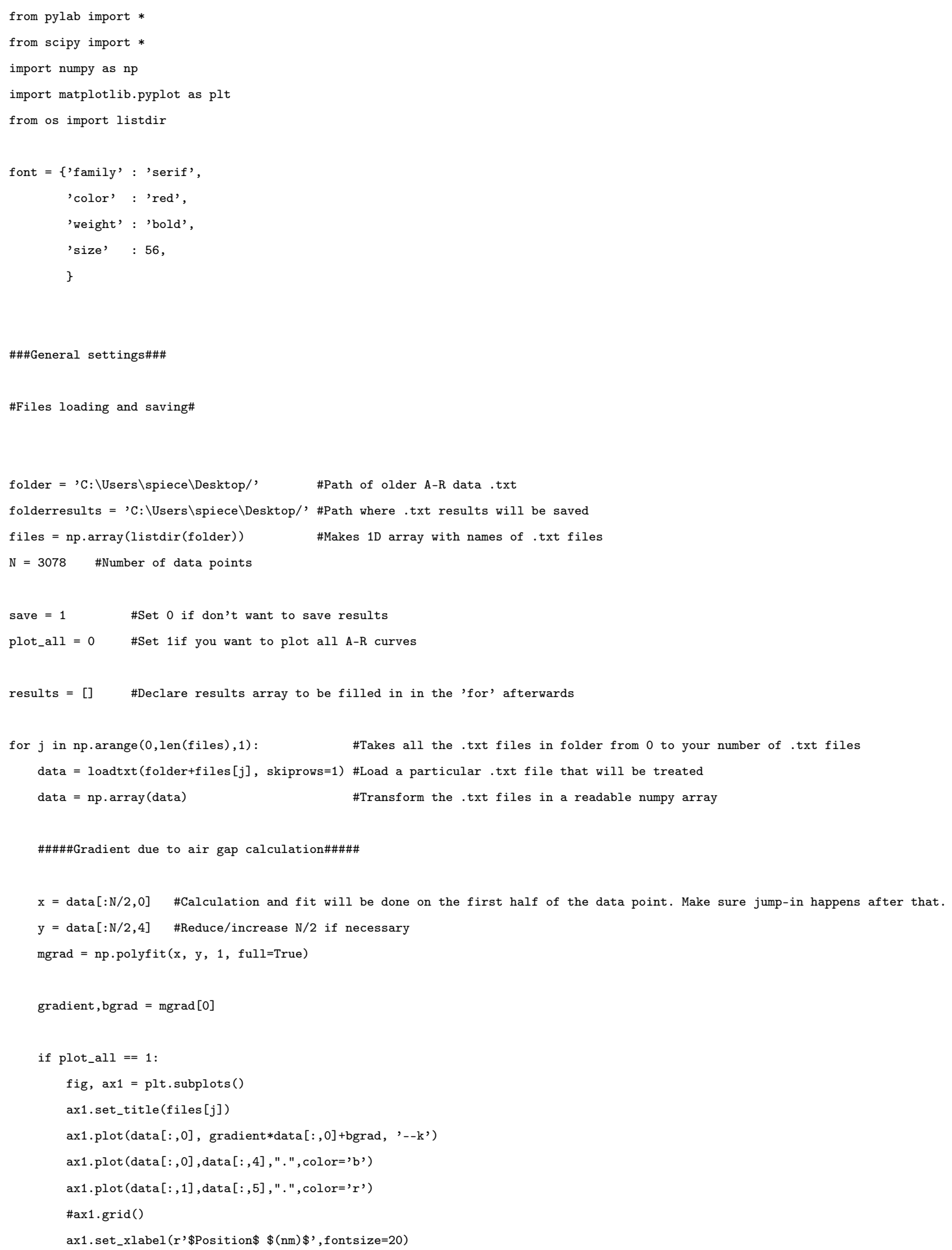




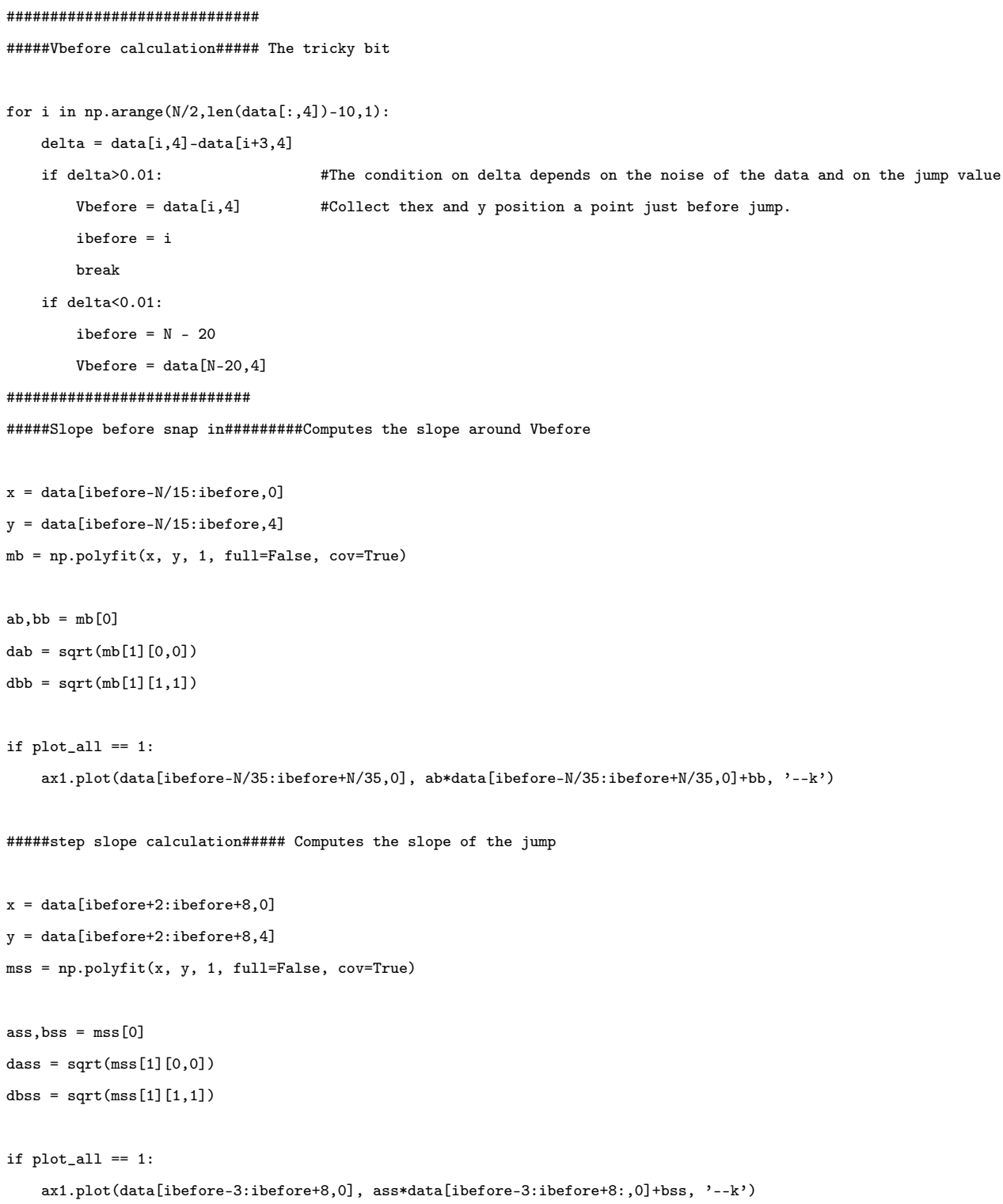

\section{\#\#\#\#\#\#\#\#\#\#\#\#\#\#\#\#\#\#\#\#\#\#\#\#\#\#\#}

\#\#\#\#gradient after calculation\#\#\#\# Computes the slope after the jump.

\#Note a choice is possible between approach or retract data

$\mathrm{x}=\operatorname{data}[: 500,1]$ \#decides if $\mathrm{V}_{-}$after is from approach or

$\mathrm{y}=\operatorname{data}[: 500,5]$ \#retract curve!

$\# \mathrm{x}=$ data $[$ ibefore+10:, 0$]$

$\# \mathrm{y}=$ data $[$ ibefore $+10:, 4]$

$\mathrm{ma}=\mathrm{np} \cdot \operatorname{polyfit}(\mathrm{x}, \mathrm{y}, 1$, full=False, cov=True $)$

$$
\mathrm{aa}, \mathrm{ba}=\mathrm{ma}[0]
$$$$
\text { daa }=\operatorname{sqrt}(\operatorname{ma}[1][0,0])
$$$$
\mathrm{dba}=\operatorname{sqrt}(\operatorname{ma}[1][1,1])
$$$$
\text { if plot_all }==1 \text { : }
$$

ax1.plot (data $[:, 0]$, aa*data $[:, 0]+b a,{ }^{\prime},-k$ ')

\section{\#\#\#\#\#\#\#\#\#\#\#\#\#\#\#\#\#\#\#\#\#\#\#\#\#\#\#}

\#\#\#\#Vbefore and Vafter recalculation\#\#\#\# Computes the actual results of Vbefore and Vafter 


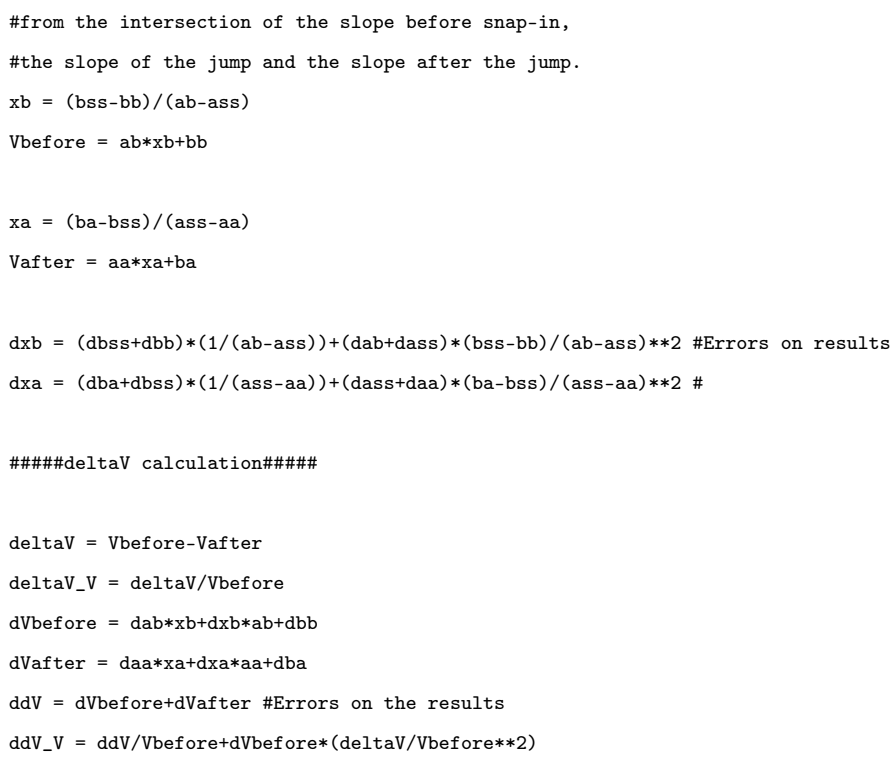




\section{HF etching of native oxide from silicon}

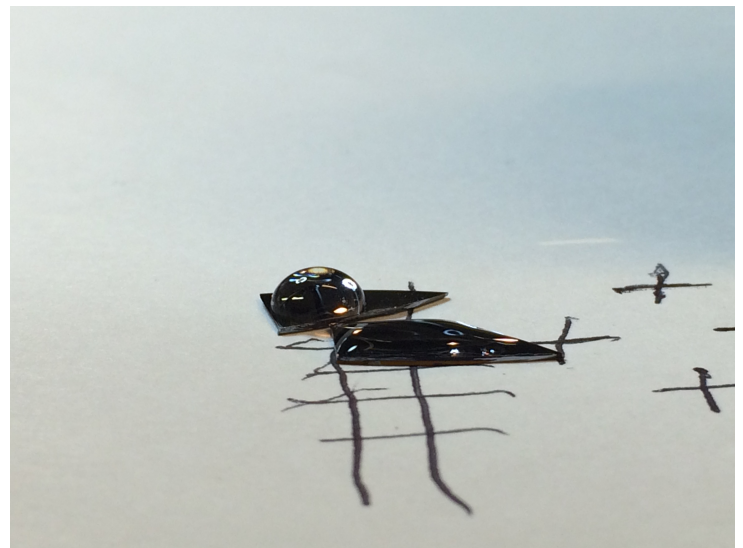

Figure A.8: HF etched and non-etched silicon wafer with water droplet showing different contact angle. 


\section{Ultrahigh precision nanolab}

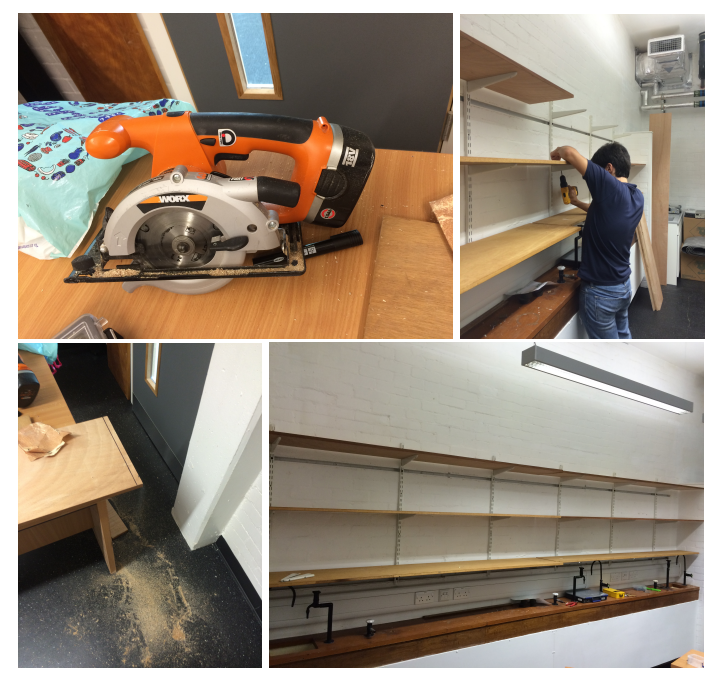

Ultra stable shelves
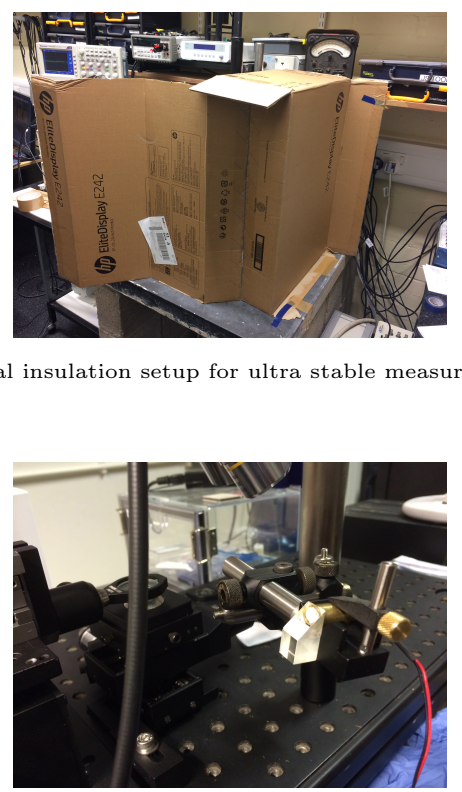

Ultra precise laser alignement setup for $2 \mathrm{D}$ materials deposition

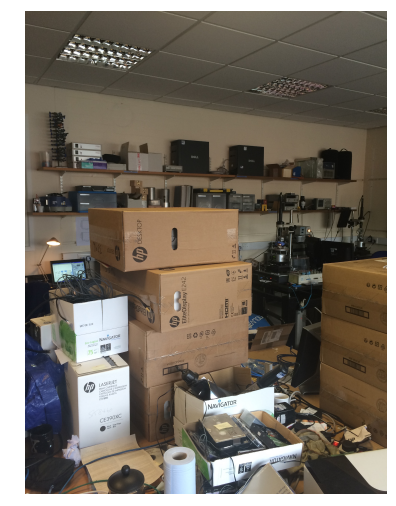

View of the lab in ultra confortable arrangement

\section{Thermal insulation setup for ultra stable measurements}

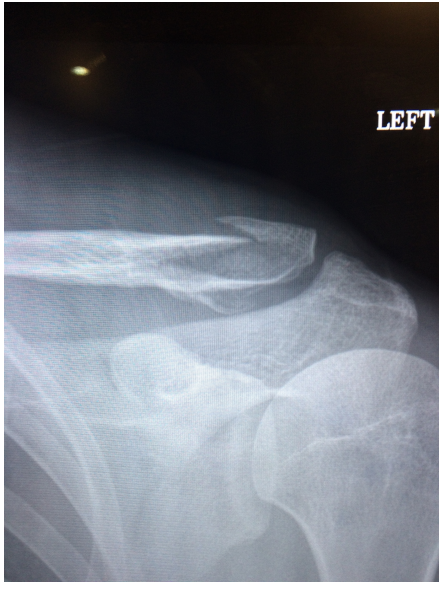

Ultra-nanofractured clavicle 


\section{Bibliography}

(1) Binnig, G.; Rohrer, H. Helvetica Physica Acta 1982, 55, 726-735.

(2) Binnig, G.; Quate, C. F.; Gerber, C. Physical Review Letters 1986, 56, 930-933.

(3) Williams, C. C.; Wickramasinghe, H. K. Applied Physics Letters 1986, 49, $1587-1589$.

(4) Kolosov, O.; Yamanaka, K. Japanese Journal of Applied Physics Part 2Letters 1993, 32, L1095-L1098.

(5) Majumdar, A. Nat Nano 2009, 4, 214-215.

(6) Chen, G. International Journal of Thermal Sciences 2000, 39, 471-480.

(7) Cahill, D. G.; Ford, W. K.; Goodson, K. E.; Mahan, G. D.; Majumdar, A.; Maris, H. J.; Merlin, R.; Phillpot, S. R. Journal of Applied Physics 2003, 93, 793-818.

(8) Gomes, S.; Assy, A.; Chapuis, P.-O. physica status solidi (a) 2015, 212, $477-494$.

(9) Abad, B.; Borca-Tasciuc, D.-A.; Martin-Gonzalez, M. Renewable and Sustainable Energy Reviews 2017, 76, 1348-1370.

(10) Lyeo, H. K.; Cahill, D. G. Physical Review B 2006, 73.

(11) Zhu, G.; Liu, J.; Zheng, Q.; Zhang, R.; Li, D.; Banerjee, D.; Cahill, D. G. Nature communications 2016, 7, 13211.

(12) Hofmeister, A. M.; Whittington, A. G. Journal of Non-Crystalline Solids 2012, 358, 1072-1082. 
(13) Shahil, K. M.; Balandin, A. A. Nano letters 2012, 12, 861-867.

(14) Freedman, J. P.; Leach, J. H.; Preble, E. A.; Sitar, Z.; Davis, R. F.; Malen, J. A. Scientific reports 2013, 3.

(15) Regner, K. T.; Sellan, D. P.; Su, Z.; Amon, C. H.; McGaughey, A. J.; Malen, J. A. Nature communications 2013, 4, 1640.

(16) Chen, Z.; Shan, X.; Guan, Y.; Wang, S.; Zhu, J.-J.; Tao, N. ACS nano 2015, 9, 11574-11581.

(17) Balandin, A. A. Nature Materials 2011, 10, 569-581.

(18) Malekpour, H.; Balandin, A. A. Journal of Raman Spectroscopy 2017, DOI: $10.1002 / j r s .5230$.

(19) Wagner, M. R.; Graczykowski, B.; Reparaz, J. S.; El Sachat, A.; Sledzinska, M.; Alzina, F.; Sotomayor Torres, C. M. Nano letters 2016, 16, 5661-5668.

(20) Cretin, B.; Gomes, S.; Trannoy, N.; Vairac, P. Microscale and Nanoscale Heat Transfer 2007, 10\%, 181-238.

(21) Gehring, P.; Harzheim, A.; Spiece, J.; Sheng, Y.; Rogers, G.; Evangeli, C.; Mishra, A.; Robinson, B. J.; Porfyrakis, K.; Warner, J. H.; Kolosov, O. V.; Briggs, G. A. D.; Mol, J. A. Nano Letters 2017, 17, 7055-7061.

(22) Kaviany, M., Heat transfer physics; Cambridge University Press: 2014.

(23) Kim, K.; Song, B.; Fernandez-Hurtado, V.; Lee, W.; Jeong, W.; Cui, L.; Thompson, D.; Feist, J.; Reid, M. T. H.; Garcia-Vidal, F. J.; Cuevas, J. C.; Meyhofer, E.; Reddy, P. Nature 2015, 528, 387.

(24) Volz, S., Microscale and nanoscale heat transfer; Springer: 2007.

(25) Asheghi, M.; Leung, Y.; Wong, S.; Goodson, K. Applied Physics Letters 1997, 71, 1798-1800.

(26) Prasher, R. Nano letters 2005, 5, 2155-2159.

(27) Prasher, R. S.; Phelan, P. E. Journal of Applied Physics 2006, 100. 
(28) Hoogeboom-Pot, K. M.; Hernandez-Charpak, J. N.; Gu, X.; Frazer, T. D.; Anderson, E. H.; Chao, W.; Falcone, R. W.; Yang, R.; Murnane, M. M.; Kapteyn, H. C., et al. Proceedings of the National Academy of Sciences 2015, 112, 4846-4851.

(29) Hu, Y.; Zeng, L.; Minnich, A. J.; Dresselhaus, M. S.; Chen, G. Nature nanotechnology 2015, 10, 701.

(30) Siemens, M. E.; Li, Q.; Yang, R.; Nelson, K. A.; Anderson, E. H.; Murnane, M. M.; Kapteyn, H. C. Nature materials 2010, 9, 26.

(31) Kapitza, P. Physical Review 1941, 60, 354.

(32) Pernot, G.; Stoffel, M.; Savic, I.; Pezzoli, F.; Chen, P.; Savelli, G.; Jacquot, A.; Schumann, J.; Denker, U.; Mönch, I., et al. Nature materials 2010, 9, 491.

(33) Prasher, R. Applied Physics Letters 2009, 94.

(34) Hinz, M.; Marti, O.; Gotsmann, B.; Lantz, M. A.; Durig, U. Applied Physics Letters 2008, 92, 043122.

(35) Cahill, D. G.; Braun, P. V.; Chen, G.; Clarke, D. R.; Fan, S.; Goodson, K. E.; Keblinski, P.; King, W. P.; Mahan, G. D.; Majumdar, A., et al. Applied Physics Reviews 2014, 1, 011305.

(36) Dinelli, F.; Biswas, S. K.; Briggs, G. A. D.; Kolosov, O. V. Physical Review B 2000, 61, 13995-14006.

(37) Dinelli, F.; Castell, M. R.; Ritchie, D. A.; Mason, N. J.; Briggs, G. A. D.; Kolosov, O. V. Philosophical Magazine A, Physics of Condensed Matter Structure Defects and Mechanical Properties 2000, 80, 2299-2323.

(38) Kolosov, O. V.; Castell, M. R.; Marsh, C. D.; Briggs, G. A. D.; Kamins, T. I.; Williams, R. S. Physical Review Letters 1998, 81, 1046-1049.

(39) Dinelli, F.; Biswas, S. K.; Briggs, G. A. D.; Kolosov, O. V. Applied Physics Letters 1997, 71, 1177-1179. 
(40) Nonnenmacher, M.; Wickramasinghe, H. K. Applied Physics Letters 1992, $61,168-170$.

(41) Pollock, H. M.; Hammiche, A. Journal of Physics D-Applied Physics 2001, 34, R23-R53.

(42) Majumdar, A. Annual Review of Materials Science 1999, 29, 505-585.

(43) Halbertal, D.; Cuppens, J.; Shalom, M. B.; Embon, L.; Shadmi, N.; Anahory, Y.; Naren, H.; Sarkar, J.; Uri, A.; Ronen, Y., et al. Nature 2016, 539, $407-410$.

(44) Sadat, S.; Tan, A.; Chua, Y. J.; Reddy, P. Nano Letters 2010, 10, 26132617.

(45) Mills, G.; Zhou, H.; Midha, A.; Donaldson, L.; Weaver, J. M. R. Applied Physics Letters 1998, 72, 2900-2902.

(46) Kim, K.; Chung, J.; Hwang, G.; Kwon, O.; Lee, J. S. ACS Nano 2011, 5, 8700-8709.

(47) Pylkki, R. J.; Moyer, P. J.; West, P. E. Japanese Journal of Applied Physics Part 1 1994, 33, 3785-3790.

(48) Dobson, P. S.; Weaver, J. M. R.; Mills, G. IEEE Sensors 2007, 708-711.

(49) Zhang, Y.; Dobson, P.; Weaver, J. Journal of Vacuum Science EJ Technology B 2012, 30, 010601 .

(50) Tovee, P.; Pumarol, M.; Zeze, D.; Kjoller, K.; Kolosov, O. Journal of Applied Physics 2012, 112, 114317.

(51) Varesi, J.; Majumdar, A. Applied Physics Letters 1998, 72, 37-39.

(52) Igeta, M.; Banerjee, K.; Wu, G.; Hu, C.; Majumdar, A. IEEE Electron Device Letters 2000, 21, 224-226.

(53) Dazzi, A.; Prazeres, R.; Glotin, F.; Ortega, J. Optics letters 2005, 30, $2388-2390$.

(54) Shetty, R. Trac-Trends in Analytical Chemistry 2012, 35, XIV-XV. 
(55) McConney, M. E.; Kulkarni, D. D.; Jiang, H.; Bunning, T. J.; Tsukruk, V. V. Nano letters 2012, 12, 1218-1223.

(56) Lai, J.; Perazzo, T.; Shi, Z.; Majumdar, A. Sensors and Actuators A: Physical 1997, 58, 113-119.

(57) Grosse, K. L.; Bae, M.-H.; Lian, F.; Pop, E.; King, W. P. Nature nanotechnology 2011, 6, 287-290.

(58) Xie, X.; Grosse, K. L.; Song, J.; Lu, C.; Dunham, S.; Du, F.; Islam, A. E.; Li, Y.; Zhang, Y.; Pop, E., et al. ACS nano 2012, 6, 10267-10275.

(59) Grosse, K. L.; Xiong, F.; Hong, S.; King, W. P.; Pop, E. Applied Physics Letters 2013, 102, 193503.

(60) Grossel, P.; Raphael, O.; Depasse, F.; Duvaut, T.; Trannoy, N. International Journal of Thermal Sciences 2007, 46, 980-988.

(61) Lefevre, S.; Volz, S. Review of Scientific Instruments 2005, 76.

(62) Chirtoc, M.; Filip, X.; Henry, J. F.; Antoniow, J. S.; Chirtoc, I.; Dietzel, D.; Meckenstock, R.; Pelzl, J. Superlattices and Microstructures 2004, 35, $305-314$.

(63) Cahill, D. G. Review of Scientific Instruments 1990, 61, 802-808.

(64) Soudi, A.; Dawson, R. D.; Gu, Y. ACS Nano 2011, 5, 255-262.

(65) Kim, K.; Jeong, W.; Lee, W.; Reddy, P. Acs Nano 2012, 6, 4248-4257.

(66) Chung, J.; Kim, K.; Hwang, G.; Kwon, O.; Jung, S.; Lee, J.; Lee, J. W.; Kim, G. T. Rev Sci Instrum 2010, 81, 114901.

(67) Menges, F.; Riel, H.; Stemmer, A.; Gotsmann, B. Review of Scientific Instruments 2016, 87, 074902.

(68) Hwang, G.; Kwon, O. Nanoscale 2016, 8, 5280-5290.

(69) Chung, J.; Kim, K.; Hwang, G.; Kwon, O.; Choi, Y. K.; Lee, J. S. International Journal of Thermal Sciences 2012, 62, 109-113. 
(70) Menges, F.; Riel, H.; Stemmer, A.; Gotsmann, B. Nano Lett 2012, 12, $596-601$.

(71) Menges, F.; Mensch, P.; Schmid, H.; Riel, H.; Stemmer, A.; Gotsmann, B. Nat Commun 2016, 7, 10874.

(72) Makris, A.; Haeger, T.; Heiderhoff, R.; Riedl, T. RSC Advances 2016, 6, 94193-94199.

(73) Gomes, S.; David, L.; Lysenko, V.; Descamps, A.; Nychyporuk, T.; Raynaud, M. Journal of Physics D-Applied Physics 2007, 40, 6677-6683.

(74) Juszczyk, J.; Kazmierczak-Balata, A.; Firek, P.; Bodzenta, J. Ultramicroscopy 2017, 175, 81-86.

(75) Pumarol, M. E.; Rosamond, M. C.; Tovee, P.; Petty, M. C.; Zeze, D. A.; Falko, V.; Kolosov, O. V. Nano letters 2012, 12, 2906-2911.

(76) Tortello, M.; Colonna, S.; Bernal, M.; Gomez, J.; Pavese, M.; Novara, C.; Giorgis, F.; Maggio, M.; Guerra, G.; Saracco, G., et al. Carbon 2016, 109, 390-401.

(77) Juszczyk, J.; Krzywiecki, M.; Kruszka, R.; Bodzenta, J. Ultramicroscopy 2013, 135, 95-98.

(78) Park, K.; Krivoy, E.; Nair, H.; Bank, S.; Yu, E. Nanotechnology 2015, 26, 265701.

(79) Ge, Y.; Zhang, Y.; Weaver, J. M.; Zhou, H.; Dobson, P. S. Journal of Vacuum Science \& Technology B, Nanotechnology and Microelectronics: Materials, Processing, Measurement, and Phenomena 2015, 33, 06FA03.

(80) Nelson, B. A.; King, W. P. Review of Scientific Instruments 2007, 78.

(81) Lee, B.; Kim, K.; Lee, S.; Kim, J. H.; Lim, D. S.; Kwon, O.; Lee, J. S. Nano letters 2012, 12, 4472-4476.

(82) Pratap, D.; Islam, R.; Al-Alam, P.; Randrianalisoa, J.; Trannoy, N. arXiv preprint arXiv:1710.09501 2017. 
(83) Assy, A.; Gomes, S. Nanotechnology 2015, 26, 355401.

(84) Lee, J.; Wright, T. L.; Abel, M. R.; Sunden, E. O.; Marchenkov, A.; Graham, S.; King, W. P. Journal of applied physics 2007, 101, 014906.

(85) Weisenhorn, A. L.; Hansma, P. K.; Albrecht, T. R.; Quate, C. F. Applied Physics Letters 1989, 54, 2651-2653.

(86) Hansma, H. G.; Vesenka, J.; Siegerist, C.; Kelderman, G.; Morrett, H.; Sinsheimer, R. L.; Elings, V.; Bustamante, C.; Hansma, P. K. Science 1992, 256, 1180-1184.

(87) Aigouy, L.; Lalouat, L.; Mortier, M.; Low, P.; Bergaud, C. Review of Scientific Instruments 2011, 82.

(88) Tovee, P. D.; Kolosov, O. V. Nanotechnology 2013, 24, 8.

(89) Gotsmann, B.; Lantz, M. A.; Knoll, A.; Dürig, U. In Nanotechnology, 2010; Chapter 4, pp 121-160.

(90) Gotsmann, B.; Lantz, M. A. Nat Mater 2013, 12, 59-65.

(91) Pettes, M. T.; Shi, L. Journal of Heat Transfer 2014, 136, 032401.

(92) Shi, L.; Majumdar, A. Journal of Heat Transfer 2002, 124, 329.

(93) Nguyen, K.; Merchiers, O.; Chapuis, P.-O. Journal of Quantitative Spectroscopy and Radiative Transfer 2017, 202, 154-167.

(94) Xu, J.-B.; Läuger, K.; Möller, R.; Dransfeld, K.; Wilson, I. Journal of Applied Physics 1994, 76, 7209-7216.

(95) Saci, A.; Battaglia, J. L.; De, I. IEEE Transactions on Nanotechnology 2015, 14, 1035-1039.

(96) Lefèvre, S.; Volz, S.; Saulnier, J.-B.; Fuentes, C.; Trannoy, N. Review of scientific instruments 2003, 74, 2418-2423.

(97) Klapetek, P.; Martinek, J.; Grolich, P.; Valtr, M.; Kaur, N. J. International Journal of Heat and Mass Transfer 2017, 108, 841-850. 
(98) El Sachat, A.; Reparaz, J.; Spiece, J.; Alonso, M.; Goñi, A.; Garriga, M.; Vaccaro, P.; Wagner, M.; Kolosov, O.; Torres, C. S., et al. Nanotechnology 2017, 28, 505704 .

(99) Martinek, J.; Valtr, M.; Cimrman, R.; Klapetek, P. Measurement Science and Technology 2014, 25, 044022.

(100) Ha, M.; Graham, S. Microelectronics Reliability 2012, 52, 836-844.

(101) Razavi, M.; Muzychka, Y. S.; Kocabiyik, S. Journal of Thermophysics and Heat Transfer 2016, 30, 863-879.

(102) Dryden, J. Journal of Heat Transfer 1983, 105, 408-410.

(103) Yovanovich, M. M.; Culham, J. R.; Teertstra, P. IEEE Transactions on Components, Packaging, and Manufacturing Technology: Part A 1998, 21, $168-176$.

(104) Muzychka, Y. S.; Sridhar, M. R.; Yovanovich, M. M.; Antonetti, V. W. Journal of Thermophysics and Heat Transfer 1999, 13, 489-494.

(105) Muzychka, Y. S.; Yovanovich, M. M.; Culham, J. R. Journal of Thermophysics and Heat Transfer 2004, 18, 45-51.

(106) Muzychka, Y. S.; Bagnall, K. R.; Wang, E. N. IEEE Transactions on Components, Packaging and Manufacturing Technology 2013, 3, 1826-1841.

(107) Muzychka, Y. S. Journal of Thermophysics and Heat Transfer 2014, 28, $313-319$.

(108) Ha, M. S. Thermal analysis of high power LED arrays., Ph.D. Thesis, Georgia Institute of Technology, 2009.

(109) Palisoc, A. L.; Lee, C. C. Journal of Applied Physics 1988, 64, 410-415.

(110) Palisoc, A. L.; Min, Y. J.; Lee, C. C. Journal of Applied Physics 1989, 65, 4438-4444.

(111) Muzychka, Y. S.; Yovanovich, M. M. Journal of Heat Transfer 2001, 123, 624. 
(112) Gholami, A.; Bahrami, M. Journal of Thermophysics and Heat Transfer 2014, 28, 679-686.

(113) Menges, F.; Riel, H.; Stemmer, A.; Dimitrakopoulos, C.; Gotsmann, B. Phys Rev Lett 2013, 111, 205901.

(114) Sadeghi, M. M.; Park, S.; Huang, Y.; Akinwande, D.; Yao, Z.; Murthy, J.; Shi, L. Journal of Applied Physics 2016, 119, 235101.

(115) Kim, M. M.; Giry, A.; Mastiani, M.; Rodrigues, G. O.; Reis, A.; Mandin, P. Microelectronic Engineering 2015, 148, 129-142.

(116) Masters, N. D.; Ye, W.; King, W. P. Physics of Fluids 2005, 17, 100615.

(117) Mu, L.; Li, Y.; Mehra, N.; Ji, T.; Zhu, J. ACS Appl Mater Interfaces 2017, 9, 12138-12145.

(118) Xu, D.; Zhang, Y.; Zhou, H.; Meng, Y.; Wang, S. Holzforschung 2016, 70.

(119) Dawson, A.; Rides, M.; Maxwell, A. S.; Cuenat, A.; Samano, A. R. Polymer Testing 2015, 41, 198-208.

(120) Crossley, S.; Usui, T.; Nair, B.; Kar-Narayan, S.; Moya, X.; Hirose, S.; Ando, A.; Mathur, N. D. Applied Physics Letters 2016, 108, 032902.

(121) Wielgoszewski, G.; Paletko, P.; Tomaszewski, D.; Zaborowski, M.; Jozwiak, G.; Kopiec, D.; Gotszalk, T.; Grabiec, P. Micron 2015, 79, 93-100.

(122) Poon, S.; Spièce, J.; Robson, A.; Kolosov, O. V.; Thompson, S. In 2017 IEEE International Magnetics Conference (INTERMAG), pp 1-2.

(123) Ramiandrisoa, L.; Allard, A.; Joumani, Y.; Hay, B.; Gomes, S. Review of Scientific Instruments 2017, 88, 125115.

(124) Maxim, N.; Mark, C. R.; Andrew, J. G.; Oleg, V. K.; Vladimir, G. D.; Dagou, A. Z. Journal of Physics D: Applied Physics 2017, 50, 494004.

(125) Timofeeva, M.; Bolshakov, A.; Tovee, P. D.; Zeze, D. A.; Dubrovskii, V. G.; Kolosov, O. V. Ultramicroscopy 2016, 162, 42-51. 
(126) Cahill, D. G.; Goodson, K.; Majumdar, A. Journal of Heat Transfer 2002, 124, 223-241.

(127) Assy, A.; Gomes, S. Applied Physics Letters 2015, 107, 043105.

(128) Battaglia, J. L.; Saci, A.; De, I.; Cecchini, R.; Selmo, S.; Fanciulli, M.; Cecchi, S.; Longo, M. physica status solidi (a) 2017, 214, 1600500.

(129) Kazmierczak-Balata, A.; Juszczyk, J.; Trefon-Radziejewska, D.; Bodzenta, J. Journal of Applied Physics 2017, 121, 114502.

(130) Ge, Y.; Zhang, Y.; Booth, J. A.; Weaver, J. M.; Dobson, P. S. Nanotechnology 2016, 27, 325503.

(131) Anis-ur-Rehman, M.; Maqsood, A. International journal of thermophysics 2003, 24, 867-883.

(132) Glassbrenner, C.; Slack, G. A. Physical Review 1964, 134, A1058.

(133) Jeong, C.; Datta, S.; Lundstrom, M. Journal of Applied Physics 2012, 111, 093708.

(134) Wang, X.; Huang, B. Scientific reports 2014, 4, 6399.

(135) Robinson, B. J.; Kay, N. D.; Kolosov, O. V. Langmuir 2013, 29, 77357742 .

(136) Robinson, B. J.; Kolosov, O. V. Nanoscale 2014, 6, 10806-16.

(137) Grishin, I.; Huey, B. D.; Kolosov, O. V. ACS Applied Materials \& Interfaces 2013, 5, 11441-11445.

(138) Burnham, N. A.; Colton, R. J.; Pollock, H. M. Nanotechnology 1993, 4, 64.

(139) Capella, B.; Baschieri, P.; Frediani, C.; Miccoli, P.; Ascoli, C. Ieee Engineering in Medicine and Biology Magazine 1997, 16, 58-65.

(140) Fischer-Cripps, A. C., Introduction to Contact Mechanics; Mechanical Engineering Series; Springer: 2007. 
(141) Derjaguin, B. V.; Muller, V. M.; Toporov, Y. P. Journal of Colloid and Interface Science 1975, 53, 314-326.

(142) Kiracofe, D.; Raman, A. Physical Review B 2012, 86.

(143) Al Mohtar, A.; Tessier, G.; Ritasalo, R.; Matvejeff, M.; Stormonth-Darling, J.; Dobson, P.; Chapuis, P.; Gomes, S.; Roger, J. Thin Solid Films 2017, 642, 157-162.

(144) Chien, H.-C.; Yao, D.-J.; Huang, M.-J.; Chang, T.-Y. Review of Scientific Instruments 2008, 79, 054902.

(145) Zhu, J.; Tang, D.; Wang, W.; Liu, J.; Holub, K. W.; Yang, R. Journal of Applied Physics 2010, 108, 094315.

(146) Liu, J.; Ju, S.; Ding, Y.; Yang, R. Applied Physics Letters 2014, 104, 153110.

(147) Jakubinek, M. B.; White, M. A.; Mu, M.; Winey, K. I. Applied Physics Letters 2010, 96, 083105.

(148) Juangsa, F. B.; Muroya, Y.; Ryu, M.; Morikawa, J.; Nozaki, T. Journal of Physics D: Applied Physics 2016, 49, 365303.

(149) Lysenkov, E.; Klepko, V. Journal of Engineering Physics and Thermophysics 2015, 88, 1008-1014.

(150) Hu, C.; Kiene, M.; Ho, P. S. Applied Physics Letters 2001, 79, 4121-4123.

(151) Zheng, K.; Sun, F.; Tian, X.; Zhu, J.; Ma, Y.; Tang, D.; Wang, F. ACS applied materials $\&$ interfaces 2015, 7, 23644-23649.

(152) Bodzenta, J.; Juszczyk, J.; Chirtoc, M. Review of Scientific Instruments 2013, 84, 093702.

(153) Mack, C. A. IEEE Transactions on Semiconductor Manufacturing 2011, 24, 202-207.

(154) Jeong, W.; Hur, S.; Meyhofer, E.; Reddy, P. Nanoscale and Microscale Thermophysical Engineering 2015, 19, 279-302. 
(155) Jung, E.; Hwang, G.; Chung, J.; Kwon, O.; Han, J.; Moon, Y.-T.; Seong, T.-Y. Applied Physics Letters 2015, 106, 041114.

(156) Lee, T.; Guo, X.; Shen, G.; Ji, Y.; Wang, G.; Du, J.; Wang, X.; Gao, G.; Altes, A.; Balk, L. Microelectronics Reliability 2002, 42, 1711-1714.

(157) Luo, K.; Herrick, R. W.; Majumdar, A.; Petroff, P. Applied Physics Letters 1997, 71, 1604-1606.

(158) Bosse, J. L.; Grishin, I.; Huey, B. D.; Kolosov, O. V. Applied Surface Science 2014, 314, 151-157.

(159) Robson, A. J.; Grishin, I.; Young, R. J.; Sanchez, A. M.; Kolosov, O. V.; Hayne, M. ACS Appl Mater Interfaces 2013, 5, 3241-5.

(160) Kolosov, O. V.; Grishin, I.; Jones, R. Nanotechnology 2011, 22, 8.

(161) Heiderhoff, R.; Li, H.; Riedl, T. Microelectronics Reliability 2013, 53, 14131417.

(162) Oehme, M.; Buca, D.; Kostecki, K.; Wirths, S.; Hollander, B.; Kasper, E.; Schulze, J. Journal of crystal growth 2013, 384, 71-76.

(163) Silvestri, H. H.; Bracht, H.; Hansen, J. L.; Larsen, A. N.; Haller, E. E. Semiconductor Science and Technology 2006, 21, 758.

(164) Iskandar, A.; Abou-Khalil, A.; Kazan, M.; Kassem, W.; Volz, S. Journal of Applied Physics 2015, 117, 125102.

(165) Frommhold, A.; Manyam, J.; Palmer, R. E.; Robinson, A. P. G. Microelectronic Engineering 2012, 98, 552-555.

(166) Frommhold, A.; Palmer, R. E.; Robinson, A. P. Journal of Micro/Nanolithography, MEMS, and MOEMS 2013, 12, 033003-033003.

(167) Goodson, K.; Flik, M.; Su, L.; Antoniadis, D. Journal of Heat Transfer 1994, 116, 317-324.

(168) Chen, J.; Zhang, G.; Li, B. Journal of Applied Physics 2012, 112, 064319. 
(169) Bhargava, N.; Gupta, J. P.; Faleev, N.; Wielunski, L.; Kolodzey, J. Journal of Electronic Materials 2017, 46, 1620-1627.

(170) Kormos, L.; Kratzer, M.; Kostecki, K.; Oehme, M.; Sikola, T.; Kasper, E.; Schulze, J.; Teichert, C. Surface and Interface Analysis 2017, 49, 297-302.

(171) Wang, W.; Li, L.; Zhou, Q.; Pan, J.; Zhang, Z.; Tok, E. S.; Yeo, Y.-C. Applied Surface Science 2014, 321, 240-244.

(172) Mahmodi, H.; Hashim, M. R. Materials Research Express 2016, 3, 106403.

(173) Khatami, S.; Aksamija, Z. Physical Review Applied 2016, 6.

(174) Uchida, N.; Maeda, T.; Lieten, R. R.; Okajima, S.; Ohishi, Y.; Takase, R.; Ishimaru, M.; Locquet, J.-P. Applied Physics Letters 2015, 107, 232105.

(175) Cheaito, R.; Duda, J. C.; Beechem, T. E.; Hattar, K.; Ihlefeld, J. F.; Medlin, D. L.; Rodriguez, M. A.; Campion, M. J.; Piekos, E. S.; Hopkins, P. E. Phys Rev Lett 2012, 109, 195901.

(176) Aksamija, Z.; Knezevic, I. Physical Review B 2013, 88.

(177) Chen, J.; Zhang, G.; Li, B. Applied Physics Letters 2009, 95, 073117.

(178) Garg, J.; Bonini, N.; Kozinsky, B.; Marzari, N. Phys Rev Lett 2011, 106, 045901.

(179) Ni, Y.; Le Khanh, H.; Chalopin, Y.; Bai, J.; Lebarny, P.; Divay, L.; Volz, S. Applied Physics Letters 2012, 100, 193118.

(180) Geim, A. K.; Grigorieva, I. V. Nature 2013, 499, 419.

(181) Dean, C. R.; Young, A. F.; Meric, I.; Lee, C.; Wang, L.; Sorgenfrei, S.; Watanabe, K.; Taniguchi, T.; Kim, P.; Shepard, K. L., et al. Nature nanotechnology 2010, 5, 722 .

(182) Mayorov, A. S.; Gorbachev, R. V.; Morozov, S. V.; Britnell, L.; Jalil, R.; Ponomarenko, L. A.; Blake, P.; Novoselov, K. S.; Watanabe, K.; Taniguchi, T., et al. Nano letters 2011, 11, 2396-2399. 
(183) Tielrooij, K.-J.; Hesp, N. C.; Principi, A.; Lundeberg, M. B.; Pogna, E. A.; Banszerus, L.; Mics, Z.; Massicotte, M.; Schmidt, P.; Davydovskaya, D., et al. Nature nanotechnology 2018, 13, 41.

(184) Molina-Mendoza, A. J.; Giovanelli, E.; Paz, W. S.; Niño, M. A.; Island, J. O.; Evangeli, C.; Aballe, L.; Foerster, M.; Van Der Zant, H. S.; RubioBollinger, G., et al. Nature communications 2017, 8, 14409.

(185) Velický, M.; Toth, P. S.; Rakowski, A. M.; Rooney, A. P.; Kozikov, A.; Woods, C. R.; Mishchenko, A.; Fumagalli, L.; Yin, J.; Zólyomi, V., et al. Nature communications 2017, 8, 14410.

(186) Ray, K.; Yore, A. E.; Mou, T.; Jha, S.; Smithe, K. K.; Wang, B.; Pop, E.; Newaz, A. ACS nano 2017, 11, 6024-6030.

(187) Bae, M.-H.; Li, Z.; Aksamija, Z.; Martin, P. N.; Xiong, F.; Ong, Z.-Y.; Knezevic, I.; Pop, E. Nature communications 2013, 4, 1734.

(188) Jang, W.; Chen, Z.; Bao, W.; Lau, C. N.; Dames, C. Nano letters 2010, 10, 3909-3913.

(189) Zhang, J.; Hong, Y.; Yue, Y. Journal of Applied Physics 2015, 117, 134307.

(190) Zhang, Z.; Xie, Y.; Peng, Q.; Chen, Y. Nanotechnology 2015, 26, 375402.

(191) Pizzocchero, F.; Gammelgaard, L.; Jessen, B. S.; Caridad, J. M.; Wang, L.; Hone, J.; Bøggild, P.; Booth, T. J. Nature communications 2016, 7, 11894.

(192) Zhang, X.; Sun, D.; Li, Y.; Lee, G.-H.; Cui, X.; Chenet, D.; You, Y.; Heinz, T. F.; Hone, J. C. ACS applied materials \& interfaces 2015, 7, 2592325929.

(193) Judek, J.; Gertych, A. P.; Świniarski, M.; Łapińska, A.; Dużyńska, A.; Zdrojek, M. Scientific reports 2015, 5, 12422.

(194) Yasaei, P.; Foss, C. J.; Karis, K.; Behranginia, A.; El-Ghandour, A. I.; Fathizadeh, A.; Olivares, J.; Majee, A. K.; Foster, C. D.; Khalili-Araghi, F., et al. Advanced Materials Interfaces 2017, 4. 
(195) Yalon, E.; Aslan, Ö. B.; Smithe, K. K.; McClellan, C. J.; Suryavanshi, S. V.; Xiong, F.; Sood, A.; Neumann, C. M.; Xu, X.; Goodson, K. E., et al. ACS applied materials $\&$ interfaces 2017, 9, 43013-43020.

(196) Chen, Z.; Jang, W.; Bao, W.; Lau, C.; Dames, C. Applied Physics Letters 2009, 95, 161910 .

(197) Mak, K. F.; Lui, C. H.; Heinz, T. F. Applied Physics Letters 2010, 97, 221904 .

(198) Kaur, S.; Raravikar, N.; Helms, B. A.; Prasher, R.; Ogletree, D. F. Nature communications 2014, 5, 3082.

(199) Xu, J.; Fisher, T. S. International Journal of Heat and Mass Transfer 2006, 49, 1658-1666.

(200) McNamara, A. J.; Joshi, Y.; Zhang, Z. M. International Journal of Thermal Sciences 2012, 62, 2-11.

(201) Yang, D. J.; Zhang, Q.; Chen, G.; Yoon, S. F.; Ahn, J.; Wang, S. G.; Zhou, Q.; Wang, Q.; Li, J. Q. Physical Review B 2002, 66.

(202) Kim, P.; Shi, L.; Majumdar, A.; McEuen, P. L. Physical Review Letters $2001,87,215502$.

(203) Yu, C. H.; Shi, L.; Yao, Z.; Li, D. Y.; Majumdar, A. Nano Letters 2005, 5, 1842-1846.

(204) Fujii, M.; Zhang, X.; Xie, H. Q.; Ago, H.; Takahashi, K.; Ikuta, T.; Abe, H.; Shimizu, T. Physical Review Letters 2005, 95.

(205) Mingo, N.; Broido, D. A. Physical Review Letters 2005, 95, 4.

(206) Vera-Marun, I.; Van Den Berg, J.; Dejene, F.; Van Wees, B. Nature communications 2016, 7, 11525. 